\title{
MULTIFUNCTIONAL POLYMERIC NANOMATERIALS FOR CANCER IMAGING AND THERAPY
}

\author{
Huang Shuo
}
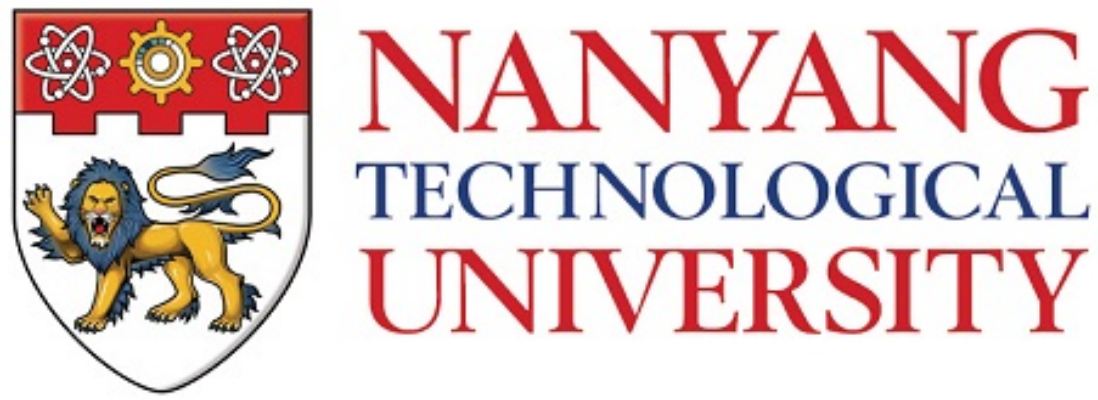

School of Chemical and Biomedical Engineering

A thesis submitted to the Nanyang Technological University in fulfilment of the requirement for the degree of Doctor of Philosophy 


\section{Acknowledgement}

Foremost, I would like to express my sincere gratitude to my supervisor Prof. Mingfeng Wang for the continuous support of my Ph.D study and research, for his patience, motivation, enthusiasm, and immense knowledge. His guidance helped me in all the time of research and writing of my thesis. I could not have imagined having a better advisor and mentor for my Ph.D study.

Secondly, I would like to thank my fellow labmates in our group: Kai Wang, Xiaochen Wang, Cangjie Yang, Wei Ren, Jinjun Shao, Jing Huang, Hui Liu, Varun Kumar Singh and Tao Jia for the stimulating discussions, for the slecpless nights we were working together, and for all the fun we have had in the two years. In particular, I am grateful to Prof. Yue Wang for enlightening me the first glance of research.

Last but not the least, I would like to thank my family: my parents for giving birth to me at the first place and also my wife and my son for always loving, encouraging and supporting me spiritually throughout my life. 


\title{
Table of Contents
}

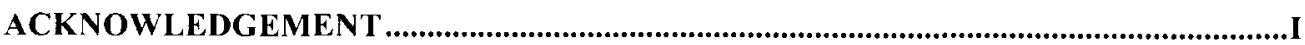

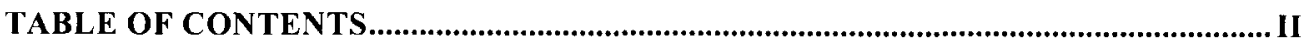

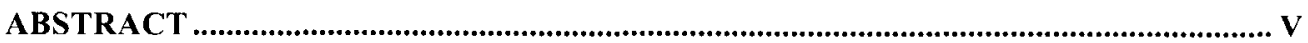

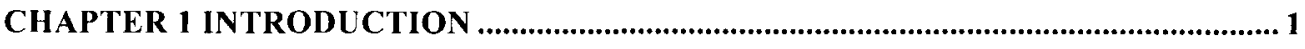

1.1 INTRODUCTION OF POLYMER NANOMATERIALS IN BIOMEDICINE. ............................. 1

1.1.1 Development of polymers in biomedicine ...................................................... I

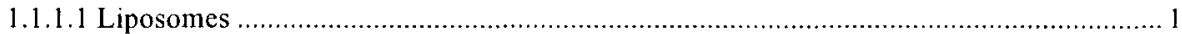

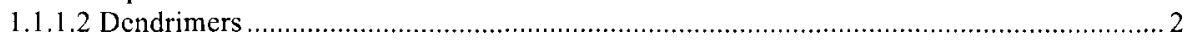

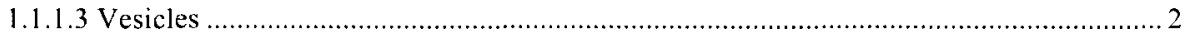

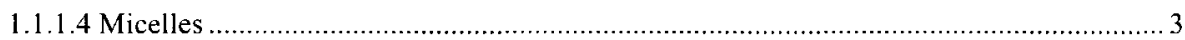

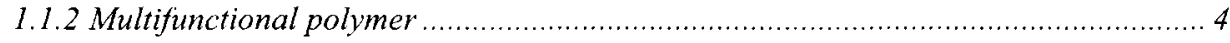

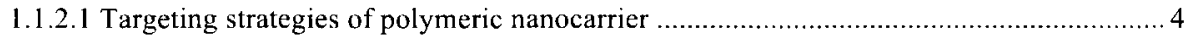

1.1.2.2 Controlled release of cargo in multi-responsive polymeric nanocarrier ............................5

1.1.2.3 Biocompatible and biodegradable polymers for biomedicine .......................................

1.1.3 Synthesis and preparation of polymeric nanomaterials ............................... 11

1.1.4 Applications of polymeric nanomaterials on cancer therapy ........................... 12

1.1.4.1 Amphiphilic polymer as nanocarrier of drug delivery system .................................. 12

1.1.4.2 $\pi$-conjugated polymers as therapeutic agents for cancer therapy ................................ 14

1.2 POLYMERIC NANOMATERIALS AS OPTICAL IMAGING PROBES ..................................... 16

1.2.1 Approaches of optical imaging ............................................................. 16

1.2.2 Organic materials of optical imaging ..................................................... 19

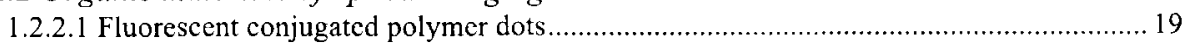

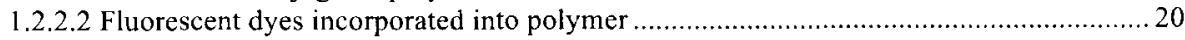

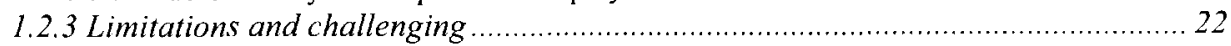

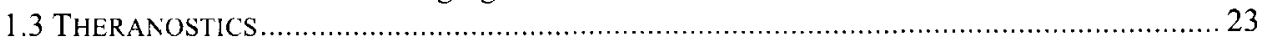

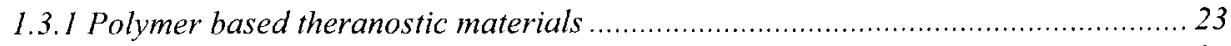

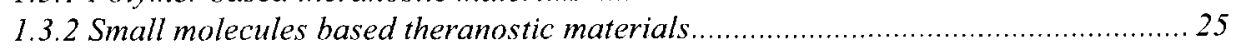

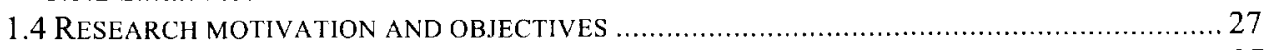

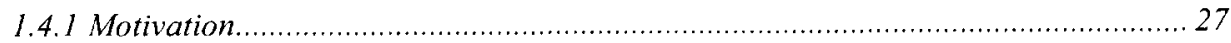

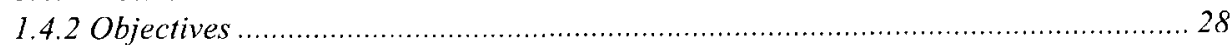

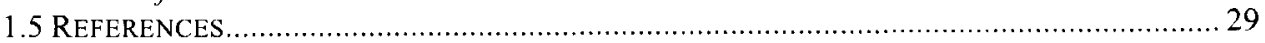

\section{CHAPTER 2 HIGHLY FLUORESCENT AND BIORESORBABLE POLYMERIC NANOPARTICLES WITH ENHANCED PHOTOSTABILITY FOR CELL IMAGING ...............................................................................45}

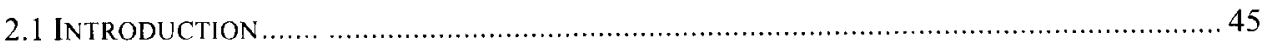

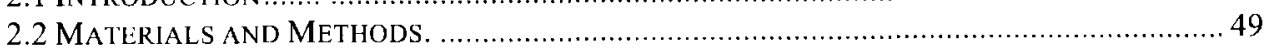

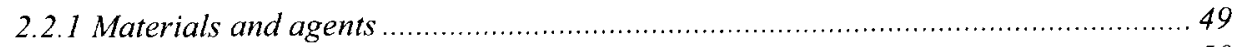

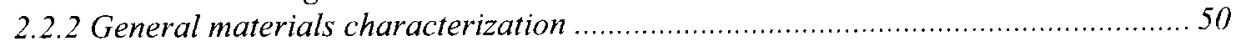

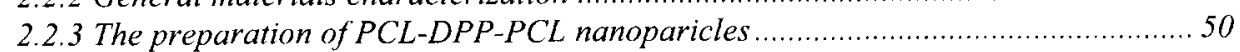

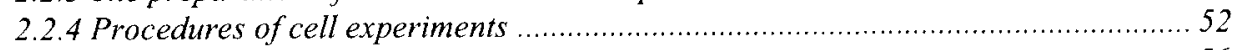

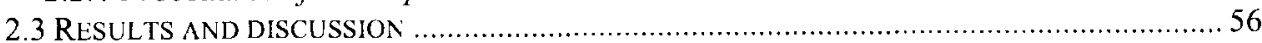

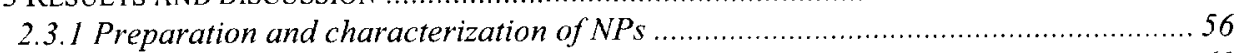

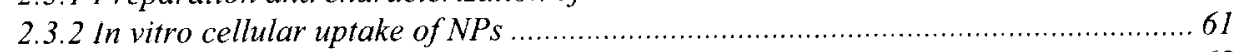

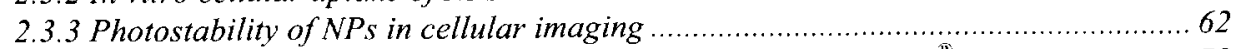

2.3.4 Long-term cell tracing using PCL-DPP-PCL NPs vs. Qtracker ${ }^{\circledR} \ldots \ldots \ldots \ldots \ldots \ldots \ldots \ldots . . . . . . . . . . . . . . . .78$

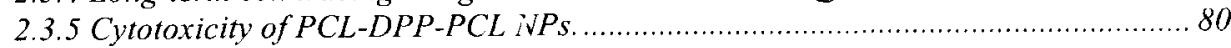


CHAPTER 3 HIGHLY FLUORESCENT POLYCAPROLACTONES WITH TUNABLE LIGHT EMISSION WAVELENGTHS ACROSS VISIBLE TO NIR

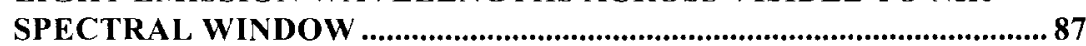

3.1 INTRODUCTION

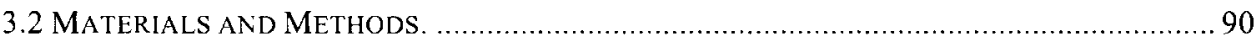

3.2.1 Materials and characterization ..................................................................... 90

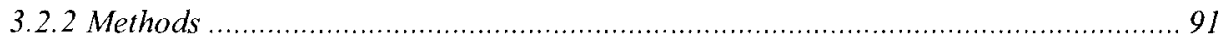

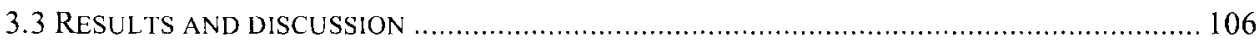

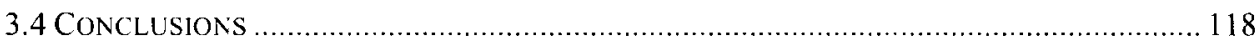

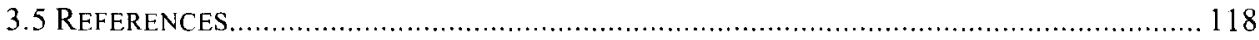

CHAPTER 4 FLUORESCENT AMPHIPHILIC BLOCK COPOLYMER FOR IMAGING PROBE AND DRUG CARRIER ........................................... 122

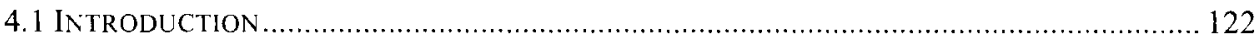

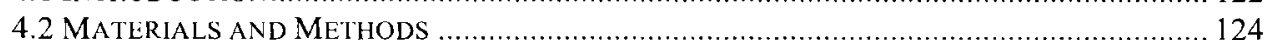

4.2.1 Materials and characterization ................................................................ 124

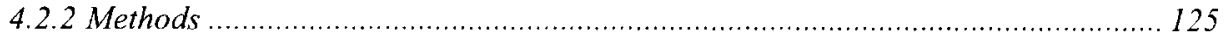

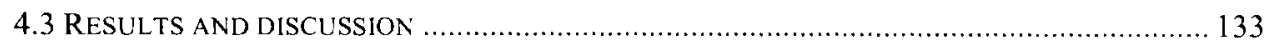

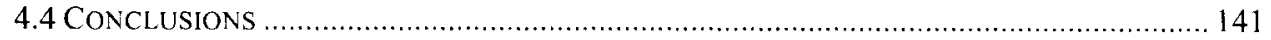

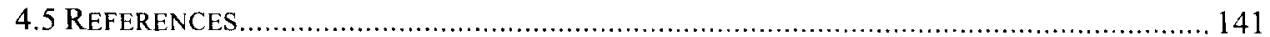

CHAPTER 5 A NARROW-BANDGAP BENZOBISTHIADIAZOLE DERIVATIVE WITH HIGH NEAR-INFRARED PHOTOTHERMAL CONVERSION EFFICIENCY AND ROBUST PHOTOSTABILITY FOR CANCER THERAPY

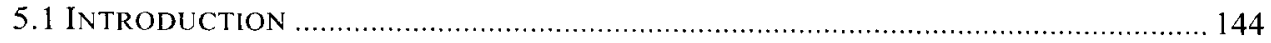

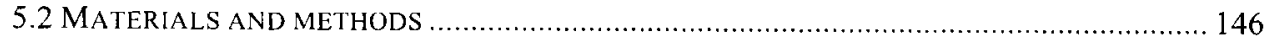

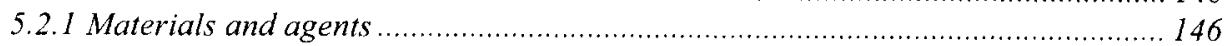

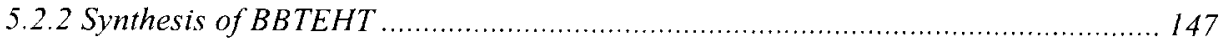

5.2.3 General materials characterization: ....................................................... 149

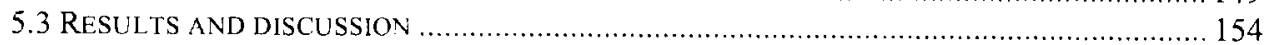

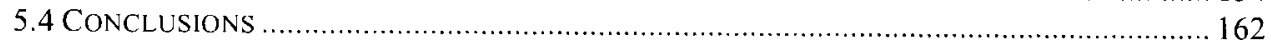

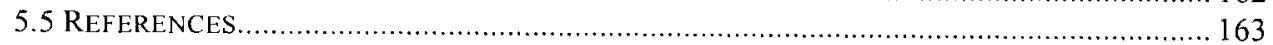

CHAPTER 6 A DUAL-FUNCTIONAL BENZOBISTHIADIAZOLE DERIVATIVE AS AN EFFECTIVE THERANOSTIC AGENT FOR NEAR-INFRARED PHOTOACOUSTIC IMAGING AND PHOTOTHERMAL THERAPY 168

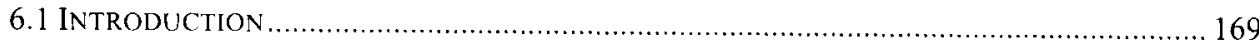

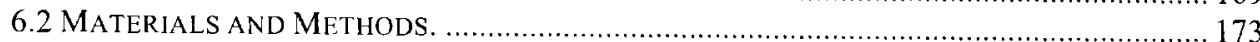

6.2.1 Materials and characterization .............................................................. 173

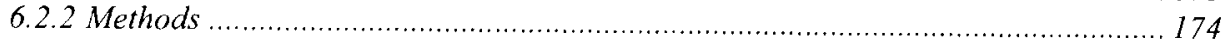

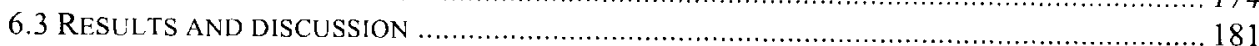

6.3.1 The preparation and characterization of BBT-2FT NPS ..................................... 181

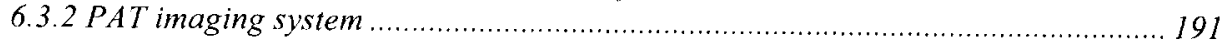

6.3.3 Photoacoustic signals from blood/BBT-2FT samples ................................... 193

6.3.4 Deep-tissue imaging experiment ............................................................... 193

6.3.5 Photothermal therapy in vitro experiments .............................................. 196

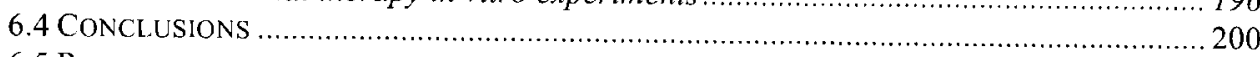

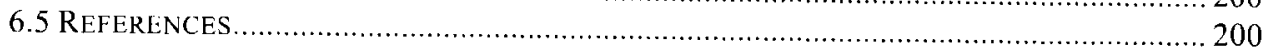

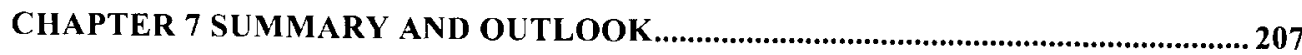




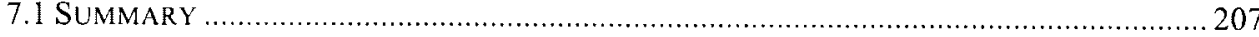

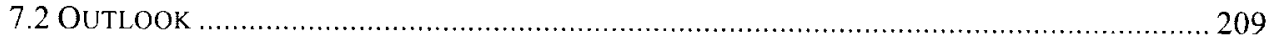

7.2.1 Amphiphilic block copolymer with NIR absorbance as theranostic materials ...... 209

7.2.2 Metal-free light triggered radical polymerization using organic photocatalysts .. 211 


\begin{abstract}
This thesis summarized my postgraduate research work on the design and synthesis of multifunctional polymers self-assembled for cancer imaging and therapy. It would focus on the fluorescent polymers from visible to near infrared (NIR) region with good biodegradability and biocompatibility that performed highly fluorescence and robust photostability for long-term bioimaging. Besides, the biocompatible polymers with NIR absorption were investigated to show excellent photothermal conversion for cancer therapy. Finally, these multifunctional polymers were explored as theranostic nanomaterials that incorporated fluorescence imaging or photoacoustic imaging with cancer therapy strategies. It could not only diagnose disease sites by strong contrast signal, but also could treat diseased tissues by releasing encapsulated drug or heat generated by photothermal effect.

In Chapter 1, I summarized the background knowledge of this thesis about polymeric nanomatcrials in biomedical as drug carrier, optical imaging probes and theranostics combining simultaneous therapy and diagnose. In addition, the challenges for current fluorescent dyes applied in optical imaging will be proposed as project motivation, including poor photostability of most organic dyes, aggregation concerns, poor signal of auto-fluorescence from tissue and low penetration depth. To overcome these challenges, this thesis will aim to develop a series of multifunctional polymeric nanomaterials for cancer imaging and therapy.
\end{abstract}


In Chapter 2, I explored a facile and novel strategy of enhancing the photostability of organic fluorophores for bioimaging applications. As a proof of concept, bright and robust fluorescence was observed in solid state of polycaprolactone consisting of di(thiophene-2-yl)-diketopyrrolopyrrole covalently linked in the middle of the polymer chain as a biocompatible and bioresorbable matrix. The polymeric nanoparticles could be internalized by both tumor cells and stem cells with little cytotoxicity. Moreover, these highly fluorescent nanoparticles exhibited significantly enhanced photostability compared to commercial quantum dots or physical blends of dye/polymer complex in cell imaging and long-term tracing.

Based on concept described in Chapter 2, a series of fluorescent and photostable polymers with various emission wavelengths across blue to near infrared (NIR) region were developed in Chapter 3. These polymers also showed strong fluorescence in solid state. Moreover, the NIR polymer could monitor the degradation of polymer scaffold by NIR fluorescence, which was consistent with mass loss.

In Chapter 4, in order to simplify the nanoparticle preparation process, hydrophilic chain was introduced to the fluorescent polymer synthesized by methods in first two chapters. So fluorescent amphiphilic block polymers were developed, which could self-assemble to nanostructures with easily controlled morphology. These fluorescent block polymers could be used as drug carrier with strong fluorescence and high encapsulation efficiency. Anti-cancer drug, doxorubicin (DOX), was encapsulated in nanoparticle matrix and could be 
released quickly in tumor condition.

Apart from strong NIR fluorescence of low band gap NIR polymers, these materials also had potential in photothermal therapy because fluorescence intensity decreased dramatically. In Chapter 5, a novel photothermal agent based on colloidal nanoparticles formed by small molecular dye, based on benzo[1,2-c;4,5-c']bis[1,2,5]thiadiazole (BBT). The nanoparticles showed strong NIR absorption, robust photostability and high therapeutic efficiency for photothermal treatment.

Based on Chapter 5, another BBT derivative (BBT-2FT) was developed with longer absorption wavelengths in Chapter 6 finally. Colloidal nanoparticles composed of BBT-2FT were developed with photoacoustic signal intensity 10 times higher than that of blood, and high photothermal conversion efficiency (47\%). 


\section{Chapter 1 Introduction}

\subsection{Introduction of polymer nanomaterials in biomedicine.}

\subsubsection{Development of polymers in biomedicine}

Polymeric nanomaterials (PNM) have been widely used in biological applications, driven by increasing innovation and emerging successes of nanotechnology. These novel polymeric materials emerged and evolved from earlier liposomes, dendrimers, vesicles and micelles to multifunctional polymers, with the purpose of control release and targeting of imaging and therapeutic agents. With respects to complex environment in biological system, multifunctional polymeric nanomaterials have been new generation products to increase drug efficiency and achieve target specificity. The flexible design of smart polymers provides more opportunities and versatile functions to increase the efficiency of diagnostic and therapeutic agents.

\subsubsection{Liposomes}

The explosion of interest in liposomes in 1960s by Bangham ${ }^{1}$ has led to rapid applications of amphiphilic nanomaterials in medical as well as food industry. The composition of phospholipids results in amphiphilic properties that can form stable nanoparticles in water, which is controlled by thermodynamic process. The hydrophilic shell can improve the solubility of the 
encapsulated drug and prevent undesirable degradation. Importantly, due to good compatibility and biodegradability, liposomes are easily metabolized in body. ${ }^{2}$ As the first gencration of nano-drug, liposomc encapsulated doxorubicin Doxil $\mathbb{R}^{3}$ has been approved by Food and Drug Administration (FDA) in 1995, which can prolong drug circulation time and reduce risk of breast cancer.

\subsubsection{Dendrimers}

Another class of polymeric nanomateirals for biomedicine is dendrimer with biomedical applications emerged in 1980 s, which refers to structurally branched macromolecules. The new concept was triggered by tree-like structure and neurons in nature to maximize their connections with each other. Compared to traditional polymer, dendrimer possesses outstanding advantages attracting many researchers. The large number of functional groups on surfaces can improve the targeting efficiency and increase signal as biosensors. ${ }^{4}$ In addition, dendrimers are more effectively to encapsulate diagnostic or therapeutic agents within the branches by hydrophobic effect or covalently bonding. Thereby it can incrcase the content loading efficiency.

\subsubsection{Vesicles}

With the development of polymer chemistry and nanotechnology, amphiphilic block polymers play essential roles in polymeric nanocarrier. In terms of the length of hydrophilic chain, it can form various nanostructures, such as vesicles, cylinder micelles and spherical micelles. Similar to liposome, 
vesicles are not only bilayer structure to encapsulate hydrophobic agents with in the shell or hydrophilic in the core, but also cnhance the stability due to higher molccular weight than liposome. Polyethylene glycol (PEG) is the widely used hydrophilic polymer chain and has been proved that it can block non-specific protein adsorption and induces no immune response. ${ }^{5}$ This property prolongs blood circulation of nanomaterials up to several days.

\subsubsection{Micelles}

When the hydrophilic chain is over $45-50$ percent (mol\%), the amphiphilic block polymers mostly yield micelles. ${ }^{108}$ In general, the amphiphilic block polymers will self assemble to core-shell structure driven by hydrophobic interaction, once the concentration is over critical micelle concentration (CMC). The stability of micelles is in terms of the van der Waals forces between the hydrophobic components, thereby the micelles will degrade when the concentration dropped below CMC. These core-shell micelles are mainly made of diblock polymers and have applications not only in pharmaceutical field, but also in coatings and personal care product. By the end of the 1990s, the triblock polymer on pharmaceutical applications emerged to attract researchers' attentions. ${ }^{164}$ In partıcular, Pluronic polymers are available commercially composed of various chain length of poly(ethyleneoxide) (PEO) and poly(propylene oxide) (PPO). Under dilute aqueous condition, Pluronic polymer self-assemble to spherical micelles with hydrophobic core. ${ }^{163}$ Due to the hydrophobic core, micelles possess excellent ability to encapsulate poor 
water-soluble drug and increase their biocompatibility. The size of micelles is around $10-200 \mathrm{~nm}$ that is much smaller than that of liposomes and vesicles. These properties of micelles lead to successful application as nanocarriers for diagnostic and therapeutic agents.

\subsubsection{Multifunctional polymer}

In order to meet the clinical requirements, many efforts have been devoted into the modification of polymeric nanomaterial. In this thesis, the cancer therapy will be mainly discussed, so polymeric nanocarrier is important to improve the efficiency of anti-cancer drug. There is a dramatically complicated blood circulation system in biological body, in which the immune system interactions and blood clearance play essential roles to avoid undesired side effect from injected nanoparticles. In addition, the kidney is capable of rapidly removing nanoparticles from the vascular compartment, and renal excretion represents a desirable pathway for nanoparticles removal with minimal catabolism. Therefore, we need to improve the efficiency of nanoparticles by prolonging the circulation time and release the anti-cancer drug at specific targeting sites precisely.

\subsubsection{Targeting strategies of polymeric nanocarrier}

Duc to the rapid devclopment of nanotechnology, significant progress has been achieved in delivery nanoparticles system. Normally, it is important to precisely deliver diagnostic agents and anti-cancer drug to target disease site, in 
order to improve pharmacokinetics and therapeutic effect. Typically, there are two kinds of strategies to increase the accumulation of nanoparticles, passive targeting and active targeting.

Passive targeting means that nanoparticles accumulate at tumor sites by their own properties and surrounding environment. Based on these enhanced vascular permeability and retention (EPR) effects in tumors, normally, the abnormal blood vessels in diseases or tumor vessels show coarse structures that the hydrodynamic diameter is less than $1 \mu \mathrm{m}$. In addition, solid tumors also have poorly organized lymphatic systems that cause low drainage and the local retention of the nanoparticles. Nanoparticles can easily penetrate the large pores of the blood vessels. Therefore, the nano-size of nanoparticles plays essential roles in passive targeting, which will have influences on pharmacokinetics and depth of penetration.

Although nanoparticles are fundamentally effective in tumor specific sites, the size dependent EPR effect based strategies alone usually suffer from the limitations of insufficiency caused by variation of tumor vascularization and internalization matter after reaching disease sites. To address this problem, researchers have attempted to develop smart polymers to improve the selectivity of the cellular uptake of nanoparticles to the targeted cells.

\subsubsection{Controlled release of cargo in multi-responsive polymeric} nanocarrier

An ideal nanoparticles for diagnostic and therapeutic agents not only need 
increased targeting efficiency of nanoparticles to targeted sites, but also require releasing the anti-cancer drug at targeted sites quickly. Controlled release of drugs from polymeric drug delivery systems is typically achieved by disassociation of micelle triggered by microenvironmet change in vivo, regulation of the rates of polymer biodegradation and drug diffusion out of the polymer matrix. ${ }^{6}$ The diffusion pathway depends on the surrounding environments and matrix property, such as porosity, swelling and degradability. On the contrary, the polymer degradation depends on the external environment, including $\mathrm{pH}$, temperature, redox or oxide state and enzymes, so the stimuli-responsive polymers play important role during this process. Figure 1-1 illustrates evolution of controlled release polymeric drug delivery systems from microscale to nanoscale spanning over 40 years. ${ }^{6}$

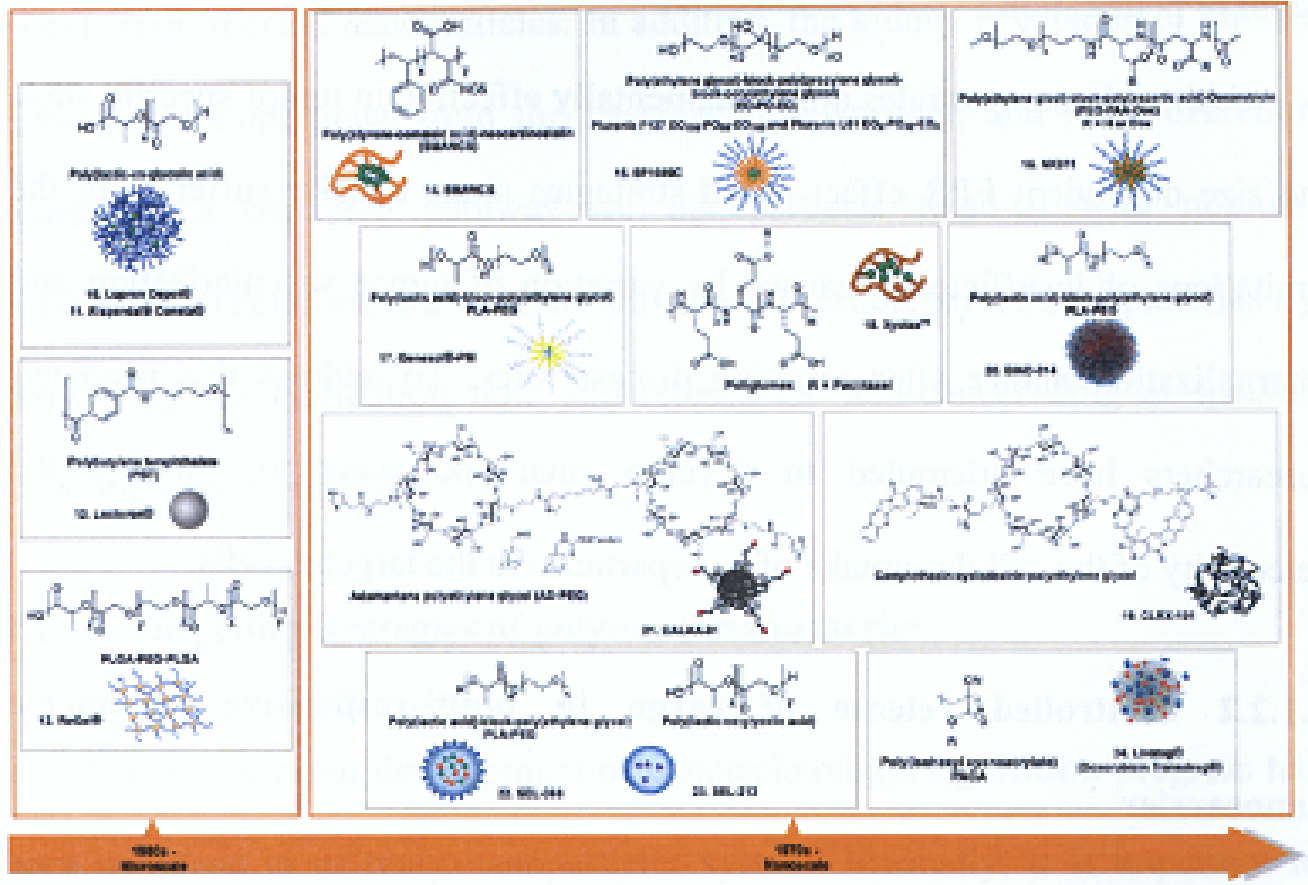

Figure 1-1. Evolution of controlled release polymeric drug delivery systems from 
microscale to nanoscale spanning over 40 years. Reprinted with permission from ref 6 . Copyright 2013 Royal Socicty of Chemistry.

Firstly, temperature-sensitive polymers have been investigated to specifically release their payloads in an induced narrow temperature range, after the delivery of a hyperthermic stimulus at the target tissue. Most of this type of polymers will change physical and chemical properties in response to heat to release the encapsulated drug. The most widcly used thermo-responsive polymers are hydrophilic below a certain temperature and become hydrophobic above a specific tempcrature, which is referred to as the lower critical solution temperature (LCST). ${ }^{7}$ Polymers that in response to temperature variation have been studied for the development of nanomedicine, including Poly(N-isopropylacrylamide) (polyNIPAAM) ${ }^{8-10}$ Poly(N-vinylcaprolactam) (polyNVCL) $)^{11-13}$ polyesters, polycarbonates ${ }^{14,15}$ and pluronic based polymers. ${ }^{16,}$ ${ }^{17}$ The temperature change range has been demonstrated that can be modulated by changing compositions or end group functionalization. ${ }^{6,165,166}$

Secondly, some endogenous factors also can trigger drug release by varying $\mathrm{pH}$ value, reducing or oxidizing species within the system. Appreciable $\mathrm{pH}$ variations in tissue and cellular level have been reported in certain pathological region, such as a notable acidic condition ( $\mathrm{pH} 4.0-6.5)$ in the extraccllular or intracellular environments of tumor tissues. ${ }^{6}$ The protonation or deprotonation of pH-sensitive polymers, such as the one connected with amine,${ }^{18}$ carboxylic amide, ${ }^{19}$ acetal groups ${ }^{20}$ and Schiff base, ${ }^{21}$ will lead to 
solubility variation of polymer and disasscmbly of nanoparticles in cellular system. ${ }^{22,23}$ In addition, Glutathione tripcptide ( $\gamma$-glutamyl-cysteinyl-glycine, $\mathrm{GSH}$ ) is a common biological reducing agent which concentration is much higher in tumor cells. ${ }^{24,25}$ Typically, the disulfide (-SS-) and diselenide (-SeSe-) bonds are reduced to thiols (-SH) when recognized by GSH agents. ${ }^{26,27}$ Attempts to synthesize reducing-sensitive polymers have achieved higher and quicker drug release. ${ }^{28-32}$ Besides, polymers can also undergo degradation in response to high levels of reactive oxygen species (ROS) found in a number of pathological conditions, which increased hydrophilicity of polymers. ${ }^{33,34}$

Last but not least, non-invasivcly remote controlled release by UV light or near infrared light can not only reduce the damages to the normal tissues but also efficiently control release of drug at specific pathological region. Since a lot of chemical and physical process can be initiated by light irradiation, resulting in propertics change of polymers. ${ }^{35-37}$ In addition, ultrasound (US) with deeper penetration depth of tissue can cause disintegration of nanoparticles, which will promote the drug release when combined with other strategies. ${ }^{38,39}$ 


\subsubsection{Biocompatible and biodegradable polymers for biomedicine}

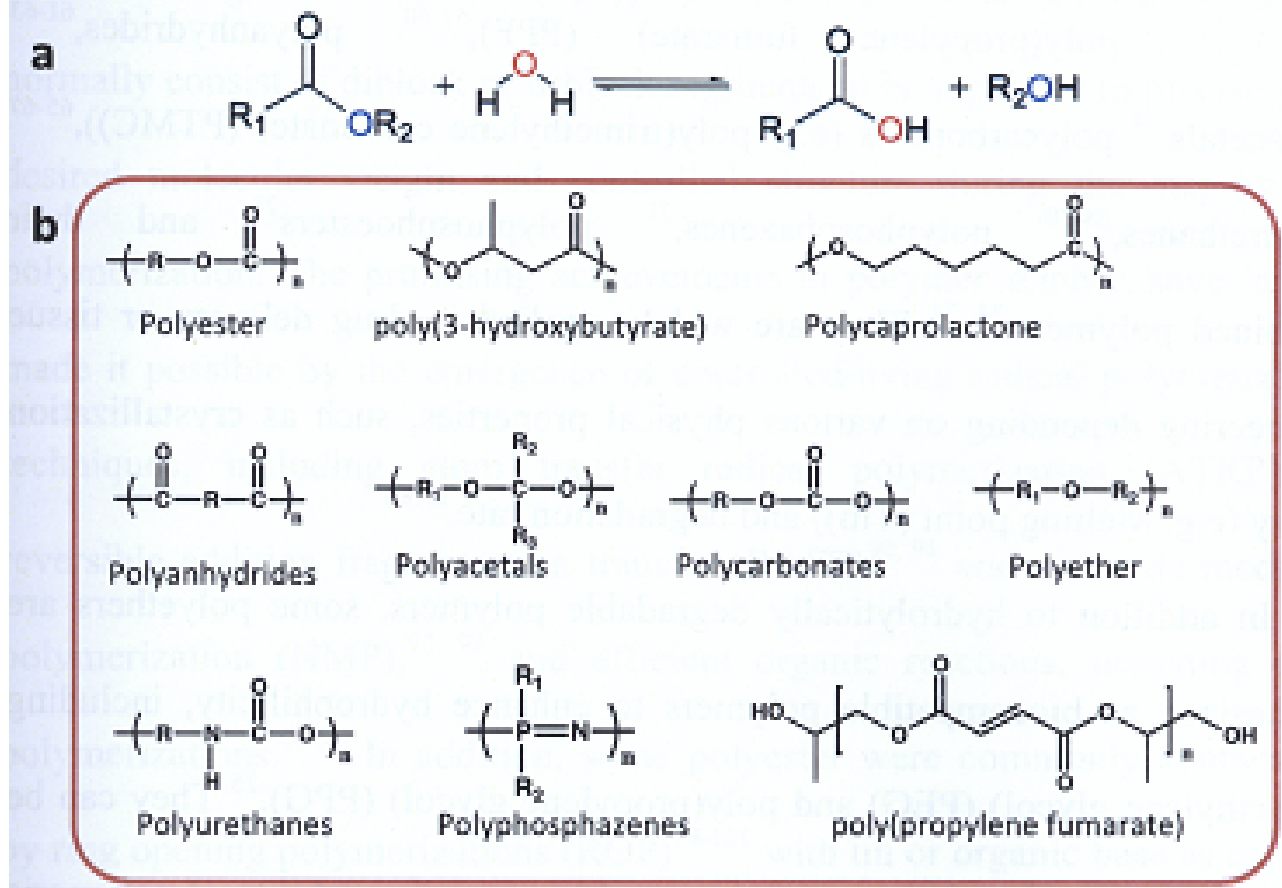

Figure 1-2. (a) Hydrolyte reaction of hydrolytically degradable polymers, which illustrate the chemical change of ester group in each repeat unit of polymer. (b) Chemical structures of some typical biodegradable polymers used in biological application.

The most important properties of polymers for clinical application are biodegradability and biocompatibility, which provide significant advantage of being able to friendly to host, bcing broken down and removed after they served their function. ${ }^{40,41}$ Among some typical biodegradable polymers (Figure 1-2), hydrolytically degradable polymers are most widely used that possess hydrolytically labile chemical bonds in their backbones. ${ }^{6,167}$ A large number of 
investigations are into polyesters, ${ }^{42-44}$ polylactic acid (PLA) or with polyglycolide, ${ }^{45-47}$ poly(3-hydroxybutyrate) $\quad$ (PHB), ${ }^{48-51}$ polycaprolactone $(\mathrm{PCL}),{ }^{52-56}$ poly(propylene fumarate) $(\mathrm{PPF}),{ }^{57-60}$ polyanhydrides, ${ }^{61-63}$ polyacetals, ${ }^{64}$ polycarbonates (e.g. poly(trimethylene carbonate) (PTMC)), ${ }^{65-67}$ polyurethanes, ${ }^{68-70}$ polyphosphazenes, ${ }^{71}$ polyphosphoesters ${ }^{72}$ and their combined polymers. ${ }^{73,74}$ They are widely applied in drug delivery or tissue engineering depending on various physical properties, such as crystallization ability (e.g. Melting point $(\mathrm{Tm}))$ and degradation rate. ${ }^{6,168}$

In addition to hydrolytically degradable polymers, some polyethers are synthesized as biocompatible polymers to enhance hydrophilicity, including poly(ethylene glycol) (PEG) and poly(propylene glycol) (PPG). ${ }^{75}$ They can be avoided from attacks of immune system and improve the stability of polymeric nanocarriers or biomolecules in clinical trials, ${ }^{76}$ however, it is recommended that higher molecular weight polyethers can cause the concerns of accumulation. $^{169}$ So some derivatives, like pluronic ${ }^{77-79}$ and PEG dimethacrylate (PEGDMA) $)^{80-82}$ etc. with high molecular weight, have been reported in a wide range of applications with greater strength and relative flexibility. Besides, the further developments of some protcins, natural/synthetic poly(amino acids) (e.g. poly(L-glutamic acid) L-PGA and poly(aspartic acid) (PAA) $)^{83}$ and polysaccharides (e.g. chitosan, hyaluronic acid $(\mathrm{HA}))^{84,85}$ have become much more common as new degradable biomaterials. 


\subsubsection{Synthesis and preparation of polymeric nanomaterials}

To design multifunctional polymers for biomedical application, which normally consist of diblock or triblock segments, it is important to precisely get desired molecular weight and controlled structure during the step-by-step polymerization. The promising achievements in polymer science have largely made it possible by the emergence of controlled/living radical polymerization techniques, including atom transfer radical polymerization (ATRP), ${ }^{86-89}$ reversible addition fragmentation transfer $(\mathrm{RAFT})^{90,91}$ and nitroxide mediated polymerization (NMP), ${ }^{92,93}$ and efficient organic reactions, including click

polymerizations. ${ }^{94-98}$ In addition, some polyester were commonly synthesized by ring opening polymerizations (ROP) ${ }^{99-101}$ with tin or organic base as catalyst. The well-defined multifunctional polymer has been successfully fabricated by a combination of these techniques. ${ }^{102}$

Polymer nanoparticles can be conveniently prepared either from preformed polymers or by direct polymerization of monomers (e.g. emulsion) on the basis of application areas, size requirement and types of polymers. In order to be used for biological application, these multifunctional polymers mostly consisting of hydrophobic and hydrophilic segments could easily form nanostructure by preformed polymers methods, such as nanoprecipitation. The hydrophilic surface of nanostructure offers robust stability in water environment and avoids undesired adsorption of immune protein during blood circulation. At meanwhile the hydrophobic core could encapsulate hydrophobic diagnostic 
molecules or anti-cancer drug, preventing them from fast excretion in body, resulting in prolonging the blood circulation.

There are a large number of methods to successfully yield a wide range of morphology of nanoparticles by tuning the ratio of hydrophobic and hydrophilic chain length, such as to prepare spheres, cylinders, lamellae, vesicles, and many other complex assemblies. Many factors can have significant effects on the morphology, such as copolymer concentration, ${ }^{103}$ good or poor solvent selection, ${ }^{104-106}$ temperature, ${ }^{107}$ water content, and presence of additives. ${ }^{108}$ Worm-like or cylinder micelles of the degradable copolymer have been evidenced to prolong circulation time compared with spherical nanoparticles. ${ }^{109-112}$ Another example claims cluster bomb like nanocarrier can achieve high penetration and accumulation efficacy in disease site than that of small micelles. ${ }^{113,114}$ When arrived disease site, the cluster micelles will degrade to a number of small nanoparticles, which is easily to be internalized into cell.

\subsubsection{Applications of polymeric nanomaterials on cancer}

\section{therapy}

\subsubsection{Amphiphilic polymer as nanocarrier of drug delivery system}

Tremendous efforts have been made in conventional drug delivery for more than 50 years, from liposomes to polymers. With the emerging of nanotechnology, one phenomenon called enhanced permeability and retention 
(EPR) effect plays a dominating role in passively targeting sites, which requires the size of nanoparticles from 20 to $200 \mathrm{~nm}$ that can leak and accumulate in diseased tissues. ${ }^{170,171}$

Another crucial requirements are the biocompatibility and biodegradability. The biodegradable copolymers mentioned above consists of hydrophilic segments and hydrophobic segments, which can encapsulate anti-cancer drugs that are insoluble in water, such as siRNA, Paclitaxel, and Doxorubicin (DOX). The hydrophobic interaction between polymers and drug is driven to form inner core of the nanoparticles, which is protected and improved stability by hydrophilic segments on the surface. For instance, PEGylated block copolymers, including PEG- $b$-PLA, PEG- $b$-PCL, Pluronic, have been shown to successfully entrap and protect delivery of anti-cancer drug. ${ }^{6}$ Similarly, drug covalently bonded in polymer chain also represents higher drug loading efficiency. ${ }^{168}$

In addition, multi-responsive polymers (c.g. $\mathrm{pH}$, temperature) that properties can be remotely controlled by environment factors are attractive to drug delivery system, since the controlled release is required to ensure effective concentration and safety of treatment. As a consequence, controlled release in drug delivery cannot only improve the effective drug concentration, but also decrease the undesired side effect of drug (Figure 1-3). ${ }^{115}$ For example, poly(N-isopropylacrylamide) (PNIPAAm) exists as hydrophilic coil below its LCST, resulting in releasing encapsulated drug. In addition, controlled release of drug can ensure to keep efficacy of drug at high level for long period than that of burst release. 


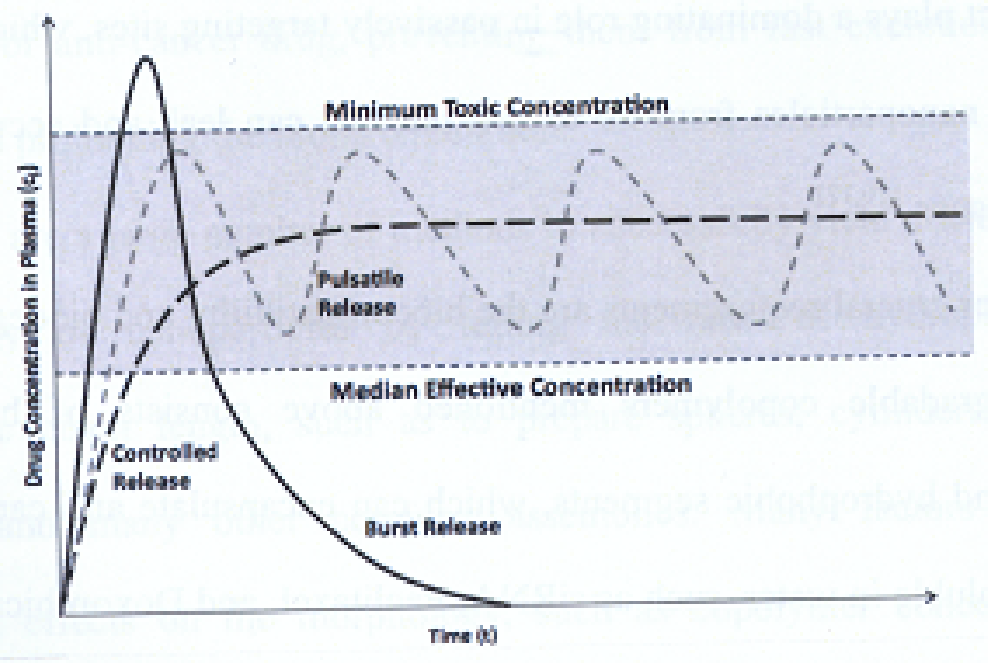

Figure 1-3. Controlled release relative to effective concentration and toxic concentration. Reprinted with permission from ref 109. Copyright 2010 Royal Society of Chemistry.

\subsubsection{2 $\pi$-conjugated polymers as therapeutic agents for cancer therapy}

Apart from some amphiphilic copolymers can be used to encapsulate treatment agents with controlled release, various conjugated polymers with strong absorbance in NIR window can also ablate cancer by photothermal effect at over $43^{\circ} \mathrm{C}$ that converts light to heat, also called hyperthermia. ${ }^{171}$ Due to high specificity, low side effect and great cfficacy, photothermal therapy has become an attractive strategy to traditional cancer therapies. Recently some inorganic materials have shown outstanding photothermal therapy efficacy, such as gold nanorods or carbon material, however, these materials with larger particle size show biodegradable concerns that remain inside body for long periods of time, resulting in serious concerns on clinical toxicity. ${ }^{172}$ Typically, the absorbance of conjugated polymers in NIR region play an important role, in which the 
polymers such as polyaniline, polypyrrole (PPy), poly(3,4ethylenedioxythiophene):poly(4-styrenesulfonate) (PEDOT:PSS) and donoracceptor (D-A) conjugated polymers (PCPDTBT, PCPDTBSe) (Figure 1-4).

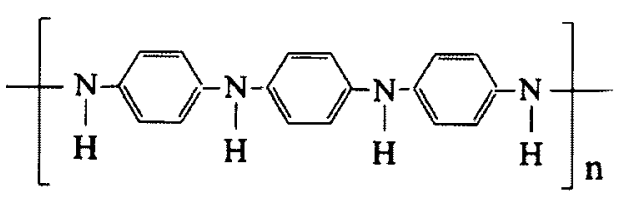

Polyaniline

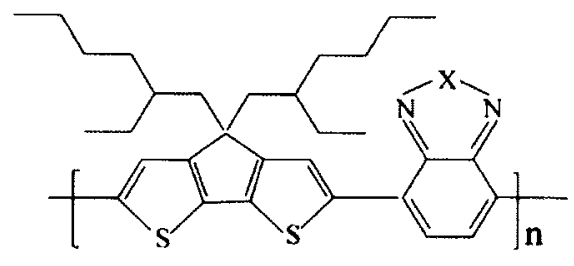

$\mathbf{X}=\mathrm{S}$, Se (PCPDTBT, PCPDTBSe)

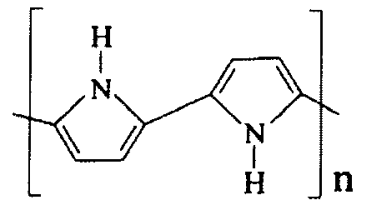

Polypyrrole

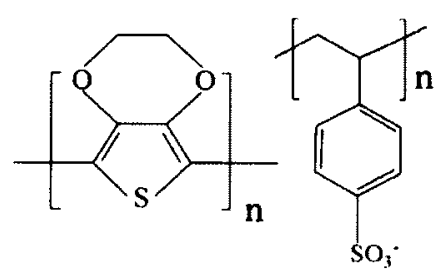

PEDOT:PSS

Figure 1-4. The chemical structures of conjugated polymers used for photothermal therapy.

These conjugated polymers had been reported a high photothermal conversion efficiency and showed excellent in vitro and in vivo phototherapeutic efficacy. ${ }^{16-123}$ These materials usually show high photostability and photothermal conversion efficiency. And they have been evidenced to be non-toxic in a number of in vitro and in vivo studies. Considering strengths and limitation of different therapeutic strategies, these conjugated polymers were also developed as drug carriers for combined cancer treatment, which details will be introduced in the following section. 


\subsection{Polymeric nanomaterials as optical imaging probes}

\subsubsection{Approaches of optical imaging}

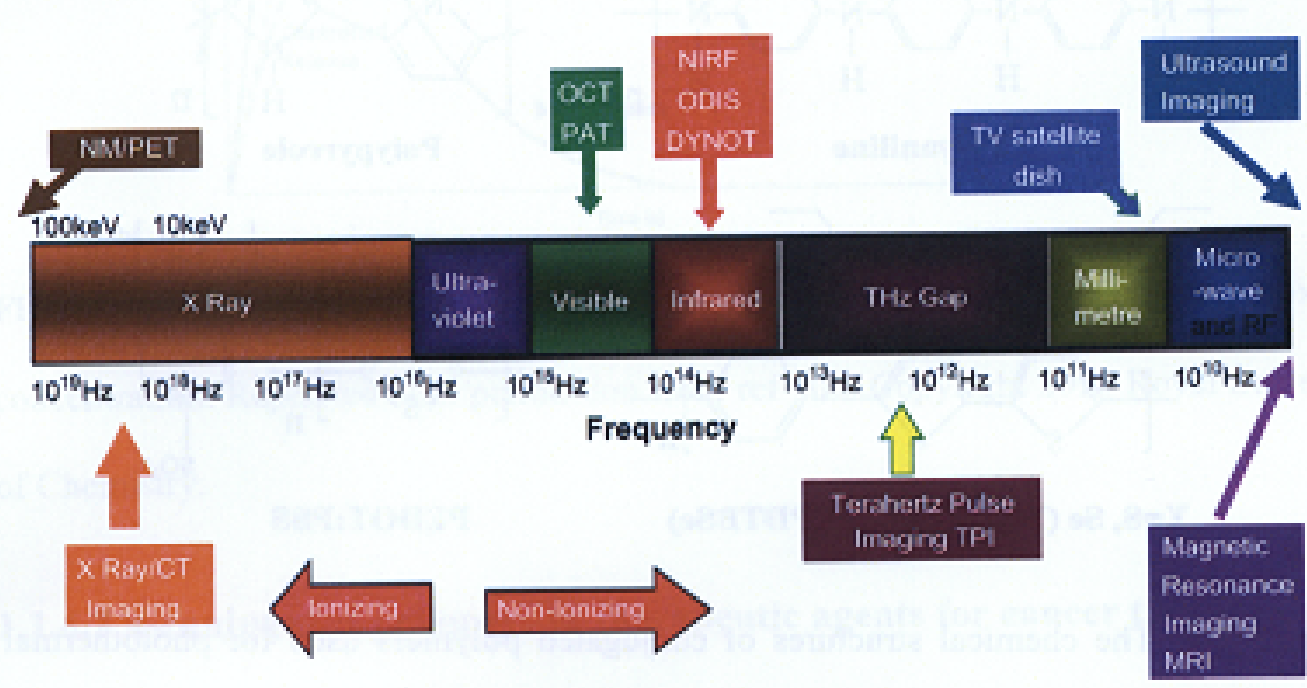

Figure 1-5. Frequency spectrum of electromagnetic radiation imaging technologies.

A large number of non-invasive imaging approaches have been described, including computcd tomography (CT), magnetic resonance imaging (MRI), positron emission tomography (PET), single photon emission CT (SPECT), ultrasound, photoacaustic imaging (PAI) and optical imaging. ${ }^{124,} 125173$ The figure 1-5 shows the frequency of various imaging technologies, in which lower frequency provided longer wavelength with deeper penetration depth for non-invasive imaging. It is crucial for in vivo bioimaging that non-invasive imaging can not only provide high-resolution signal but also avoid body damaged from imaging probes. Basically, the basic signal of MRI comes from 
the procession of water hydrogen nuclei in magnetic ficld. After radio-frequency pulse, the relaxation process of nuclei return to original state can produce image. Similarly, $\gamma$-ray emissions are the basis for SPECT and PET, ${ }^{176}$ which are acquired over a nominally low background signal and require little signal amplification since the gamma rays have energies in the megavolt range. ${ }^{174,175}$ These strategies became outstanding imaging approaches due to deep penetration and detectable signal.

However, these imaging approaches are limited by the potential toxicity of contrast agents consisting of heavy metal or non-degradable inorganic materials. ${ }^{173}$ Compared with other imaging approaches, fluorescence optical imaging is one of most common used strategies in research, which utilizes the photons emitted from bioluminescent or fluorescent probes. The development of fluorescence imaging techniques has provided effective research tools to investigate many fundamental processes in the life sciences. Various fluorescence-based agents have also been applied to monitor a broad range of biological activities, such as conformation dynamics, interactions, and distribution of biomolecules in organelles, cells, or tissues. The spectrum from visible to near-infrared (NIR) light provides good spatial resolution, without exposure to ionizing radiation. Furthermore, the high fluorescent quantum yield can provide more sensitive signal. These features provide fluorescent probes potential applications in optical imaging.

As a promising optical imaging agents, inorganic semiconductor quantum dots (QDs) have also been reported as useful cell labeling reagents, which have 
shown bright emission and high photostability. Unfortunately, commonly used QDs, such as CdSe and CdTe, contain heavy metal cores, which have shown undesired cytotoxicity and severe aggregation. ${ }^{173}$ These serious side effects hinder their applications in biological and biomedical events. Besides, organic fluorescent dyes also have potential ability in cellular imaging. A large variety of dye molecules, such as fluorescein, rhodamine, and cyanine, are available for different experiments, and their optical properties can be fine-tuncd by various design strategies. However, this modality suffers from poor tissue penetration $(0-2 \mathrm{~cm})$ and fluorescent imaging is highly susceptible to noise due to the tissue scattering of photons in the visible light region $(395-600 \mathrm{~nm}) .{ }^{126}$ Moreover, the further applications of organic dyes are always limited by aggregation caused fluorescence quenching and photobleaching. Optical imaging also suffers from significant background because of tissue autofluorescence and light absorption by proteins $(257-280 \mathrm{~nm})$, heme groups (max absorbance at $560 \mathrm{~nm}$ ), and even water (above $900 \mathrm{~nm}$ ). Thus, fluorescence imaging in vivo is mainly used for small animal studies and not generally translated into the clinic. These limitations are hopefully overcome to a great extent if a breakthrough in signal intensity is achicved by using near-infrared (NIR) (700-1100 nm) fluorescent probes. This technique uses fluorescent dyes that absorb and emit near-infrared (NIR) radiation a spectral window where hemoglobin and water absorb minimally and therefore allow photons to penetrate several centimeters through tissue. The deep penetration of NIR emission could be used in non-invasive imaging. 


\subsubsection{Organic materials of optical imaging}

\subsubsection{Fluorescent conjugated polymer dots}

Main-chain $\pi$-conjugated polymers have attracted many attentions and been applicd in solar cell materials, such as OFET and OLED. The strategy for the synthesis of fluorescent polymer nanoparticles, such like polyfluorene (PF), polyphenylencethynylene (PPE), or copolymers, is to form the particles from fluorescent $\pi$-conjugated polymers, which results in semiconducting polymer dots (Pdots). Remarkably, other $\pi$-conjugated polymers have also been used to yield particles of similar sizes, thereby suggesting that the chemical structure, molecular weight and size distribution do not have a significant influence on the particle size obtained (Figure 1-6). ${ }^{126}$ Such nanoparticles generally show bright fluorescence with high quantum yiclds up to $40 \%$ for PF-based aggregates. Owing to the different chemical nature of $\pi$-conjugated polymers, a large range of the visible spectra can be covered. These particles are very stable over time and can emit up to $10^{9}$ photons prior to photobleaching, which is between 3-4 orders of magnitude higher than that of typical fluorescent dyes. The bright fluorescence and stability make conjugated polymer to be used as imaging agents in biological application. ${ }^{127-129}$ 

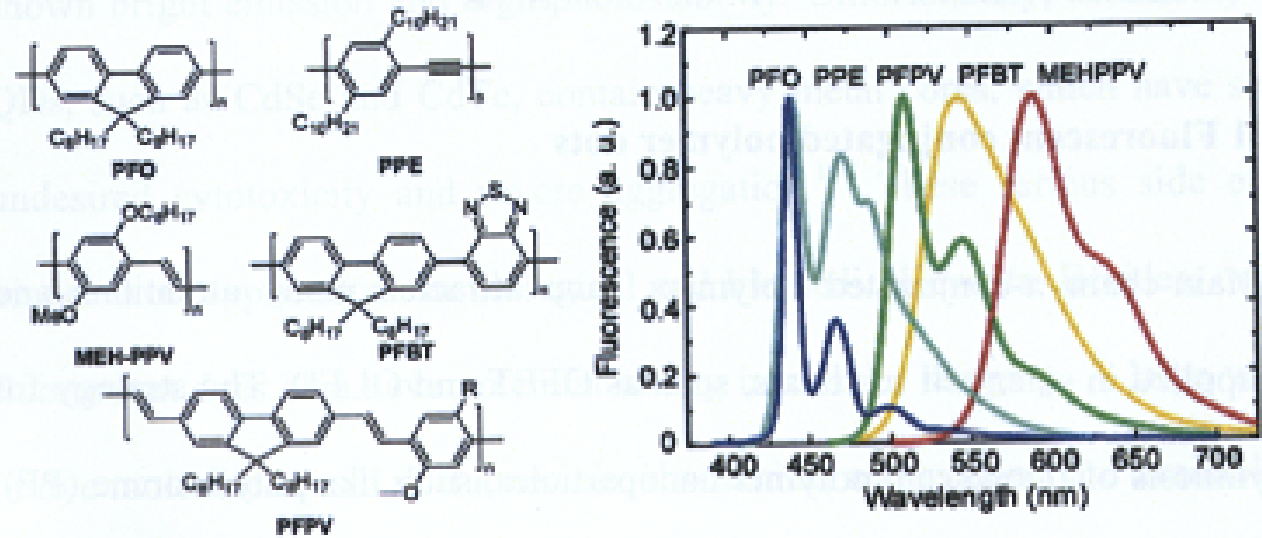

Figure 1-6. Chemical structures and their corresponding fluorescence spectra of poly(9,9-dioctylfluorenyl-2,7-diyl) $\quad$ (PFO), $\quad$ poly[2,5-di(3,7-dimethyloctyl) phenylene-1,4-ethynylene] (PPE), poly[2-methoxy-5- (2-cthylhexyloxy)- 1,4phenylenevinylene $] \quad$ (MEH-PPV), poly[(9,9- dioctylfluorenyl-2,7-diyl)-co-(1,4benzo-[2,1,3] -thiadiazole)] (PFBT) and poly[(9,9-dioctyl-2,7- divinylenefluorenylene)-alt-co-(2-methoxy-5-(2-ethylhexyloxy)-1,4-phenylene)] (PFPV). Adapted from.126. Copyright 2008 Royal Society of Chemistry.

\subsubsection{Fluorescent dyes incorporated into polymer}

The fluorophore-conjugated block copolymers were synthesized and investigated, especially the incorporation of organic dyes into polymeric architectures attracted interest due to their potential ability to address some existing drawbacks limiting organic dyes in biological system, such as biocompatibility, the stability of organic dyes, and biodistribution. In contrast to main-chain $\pi$-conjugated polymers, the optoelectronical properties of the polymer that covalently attached side-chain pendant dyes to a polymeric backbonc are mostly independent from the degree of polymerization and the 
number of repeating units, respectively. Furthermore, monomers that carry polymerizable functionalities, e.g., double bonds in terms of (meth)acrylate-, vinyl- or styrene units, are capable to be directly polymerized by one of the commonly applied controlled radical polymerization (CRP) techniques, ${ }^{178}$ in particular, by the reversible addition-fragmentation transfer (RAFT) polymerization, or the atom transfer radical polymerization (ATRP), ${ }^{177}$ or the nitroxide mediated polymerization (NMP). There have been a lot of organic dyes investigated as side chain of polymer, including BODIPY, ${ }^{130}$ 1,8-naphthalimides, fluoresceins, rhodamines, coumarins, azo-dyes, oxadiazoles, diverse aromatic dyes. ${ }^{131-136}$ However, some important group of dyes can act as radical scavenger, resulting in that these molecules are not suitable for radical polymerizations. Furthermore, they tend to phase separate more easily in block copolymer architectures. ${ }^{137}$ Moreover, the fluorescence tends to quench caused by aggregation in hydrophobic core of nanoparticles for biological applications.

In order to overcome the concern of aggregation caused fluorescence quenching, a series of small molecules with aggregation induced emission effect (AIE) has been reported by Tang and his coworkers. ${ }^{138-143}$ The polymers incorporated with AIE dyes as side or end group shows highly fluorescence signal in aggregated state, resulting in impressive progress for biological applications. These AIE polymers have been studicd for DNA detection, ${ }^{144}$ bacteria detection, ${ }^{145}$ cellular imaging ${ }^{146}$ and intracellular pH mapping. ${ }^{147}$ 


\subsubsection{Limitations and challenging}

Despite a lot of advantages of organic materials in optical imaging, there are still limitations before further clinical applications. Firstly, as most of recent commercial fluorescence imaging agents with visible emission wavelength, there is a serious concern regarding to penetration depth, since non-invasive diagnosis will be an ideal choice in clinical applications that needs deeper penetration to tissues. As a consequence, novel organic materials with NIR absorbance will be an excellent alternative to figure out this concern. Normally, emission wavelength of NIR materials is from 700 to $1100 \mathrm{~nm}$, where the tissue is transparent in this window.

Secondly, despite AIE molecules with highly fluorescence in aggregated state, it does not apply to every fluorescent dyes but only limits to a class of molecules with AIE group. Thus, aggregation caused fluorescence quenching (ACQ) is always a severe concern to organic dyes.

Last but not least, most organic fluorophores suffer from their poor photostability, i.e. the fluorescence intensity decays quickly (tens of seconds) under continuous exposure to light, which limits long-term applications. This phenomenon, also called photobleaching, limits the ability of employing fluorescence microscopy or spectroscopy for long-term tracking of biological activities. Previous studies indicate that the formation of non-fluorescent triplet states (denoted as $\mathrm{T}_{1}$ ), following the photonic excitation process, and the presence of molecular oxygen play a major role in the photobleaching of 
organic fluorophores. In air-saturated solutions, reactions between moleculk $r$ oxygen and $T_{1}$ lead to substantial generation of reactive oxygen species (RO؟), including $\mathrm{HO} \bullet, \mathrm{HO}_{2} \bullet$, and $\mathrm{H}_{2} \mathrm{O}_{2}$, and rapid photobleaching.

\subsection{Theranostics}

Polymer nanoparticle-based therapy and molecular imaging have been applied widely. However, the push provided by advances in nanotechnology and the calls for personalized medicine have already made further application called theranostic a research hotspot, which combine both therapeutic and diagnostic agents together, with emphasis on cancer. There are unique opportunities to use multifunctional formulations for both diagnostic and therapeutic purposes.

\subsubsection{Polymer based theranostic materials}

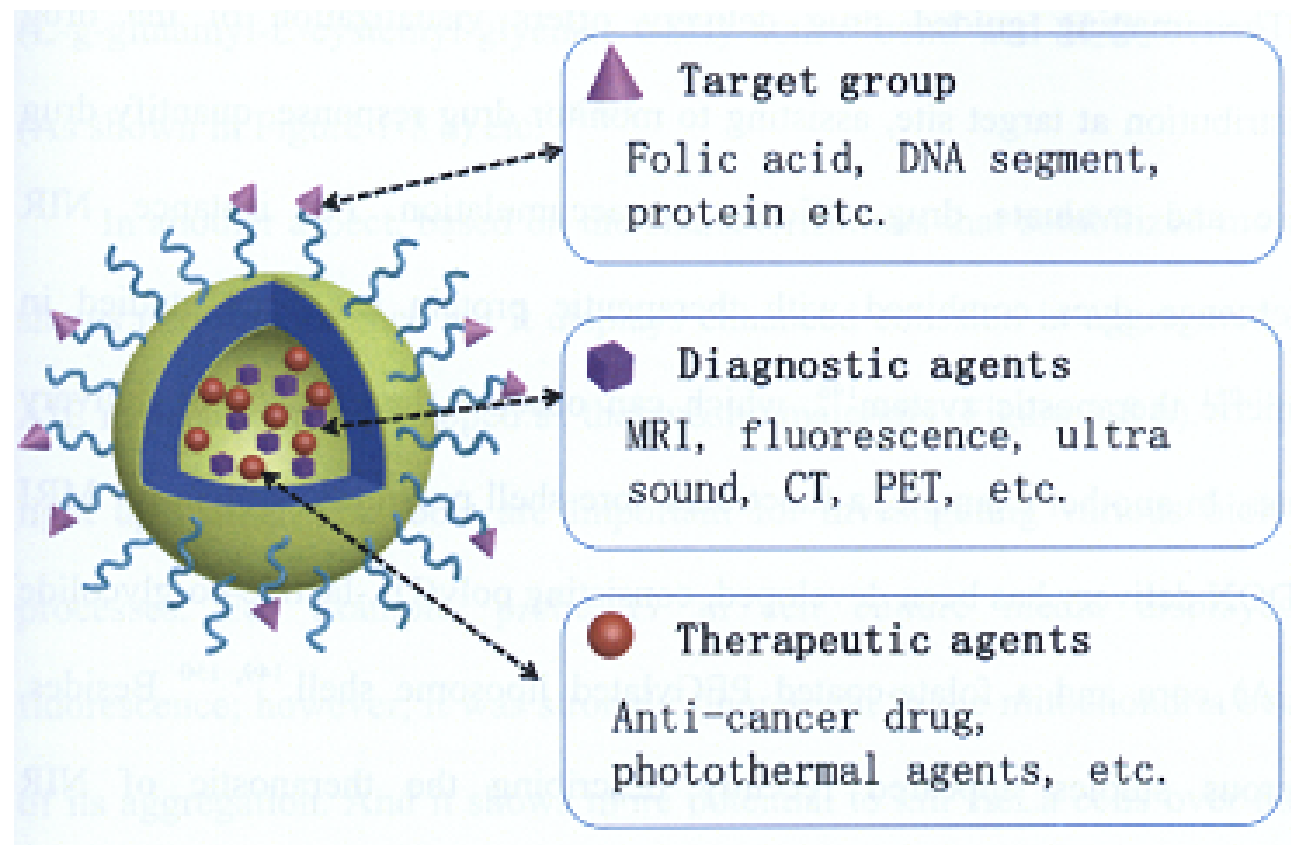


Figure 1-7. Illustration of main components that incorporated into theranostic agents.

Despite some inorganic materials, such as silica nanoparticles, could act as nanocarrier to encapsulate diagnostic agents and therapeutic agents. However, the concern of biodegradability of inorganic materials limited further clinical applications. Thus, polymeric nanoparticles have more potential applications in theranostic materials. The general polymeric materials for theranostics, as shown in figure 1-7, should have three components: (1) the polymeric nanoparticles with target group that offers good biocompatibility, (2) the therapeutic components carriers drug or gene or protein or photothermal therapy that used for disease treatment and (3) a diagnostic agents that can be used for imaging (for example contrast agents). The function of polymer nanoparticles is to provide stable environment for imaging probes and therapeutic agents.

The imaging guided drug delivery offers visualization of the drug biodistribution at target site, assisting to monitor drug response, quantify drug release and evaluate drug efficacy or accumulation. For instance, NIR fluorescence dyes combined with therapcutic protein has been studied in polymeric theranostic system ${ }^{148}$, which can observe the efficacy of delivery process. In another example, a functional core-shell polymeric system for MRI and DOX delivery has been developed, consisting poly(DL-lactide-co-glycolide) (PLGA) core and a folate-coated PEGylated liposome shell. ${ }^{149,}{ }^{150}$ Besides, numerous studies appeared recently describing the theranostic of NIR 
fluorescence imaging guided photothermal therapy. ${ }^{151,} 152$ The polymeric theranostic materials cannot only utilize the diagnostic signal from contrast agents giving precise delivery route of nanoparticles, but also some polyr aric materials, such as conjugated polymers, work as therapcutic agents to tre't disease efficiently.

\subsubsection{Small molecules based theranostic materials}

Except from polymeric materials, some fluorescent small molecules have opportunity to become excellent theranostic materials by modification with therapcutic agents. Due to the versatile functional group from small molecules, especially fluorescent dyes, it is easily to conjugate anti-cancer drug or photodynamic agents onto small molecules covalently. To achieve controlled release of therapeutic agent, except from click reaction, some biologically responsive covalent bonds have been widely used, such as Glutathione (L-g-glutamyl-L-cysteinyl-glycine; GSH) active bond and $\mathrm{H}_{2} \mathrm{O}_{2}$ active bond (As shown in Figure 1-8 a) etc. ${ }^{153-158}$

In another aspect, based on the characterizations that solubilized molecule shows non-fluorescence but it displays enhanced emission in aggregated state, AIE molecules are developed as theranostic materials (Figure 1-8 b). ${ }^{159-161}$ The light up" diagnostic tools are important for investigating various biological processes. For example, probe 17 in cell culture media displayed no fluorescence; however, it was strongly fluorescent in the mitochondria becausc of its aggregation. And it shows more potential to kill HeLa cells over normal 
cells by induced ROS overproduction. ${ }^{162}$

No matter polymeric or small molecular theranostic systems, they with salient features are emerging biomedicine interest consisting of drugs and imaging agents. By varying the drug and the target site, and by combining them with several modalities, such as optical and MRI, the versatility of theranostics might be further increased.
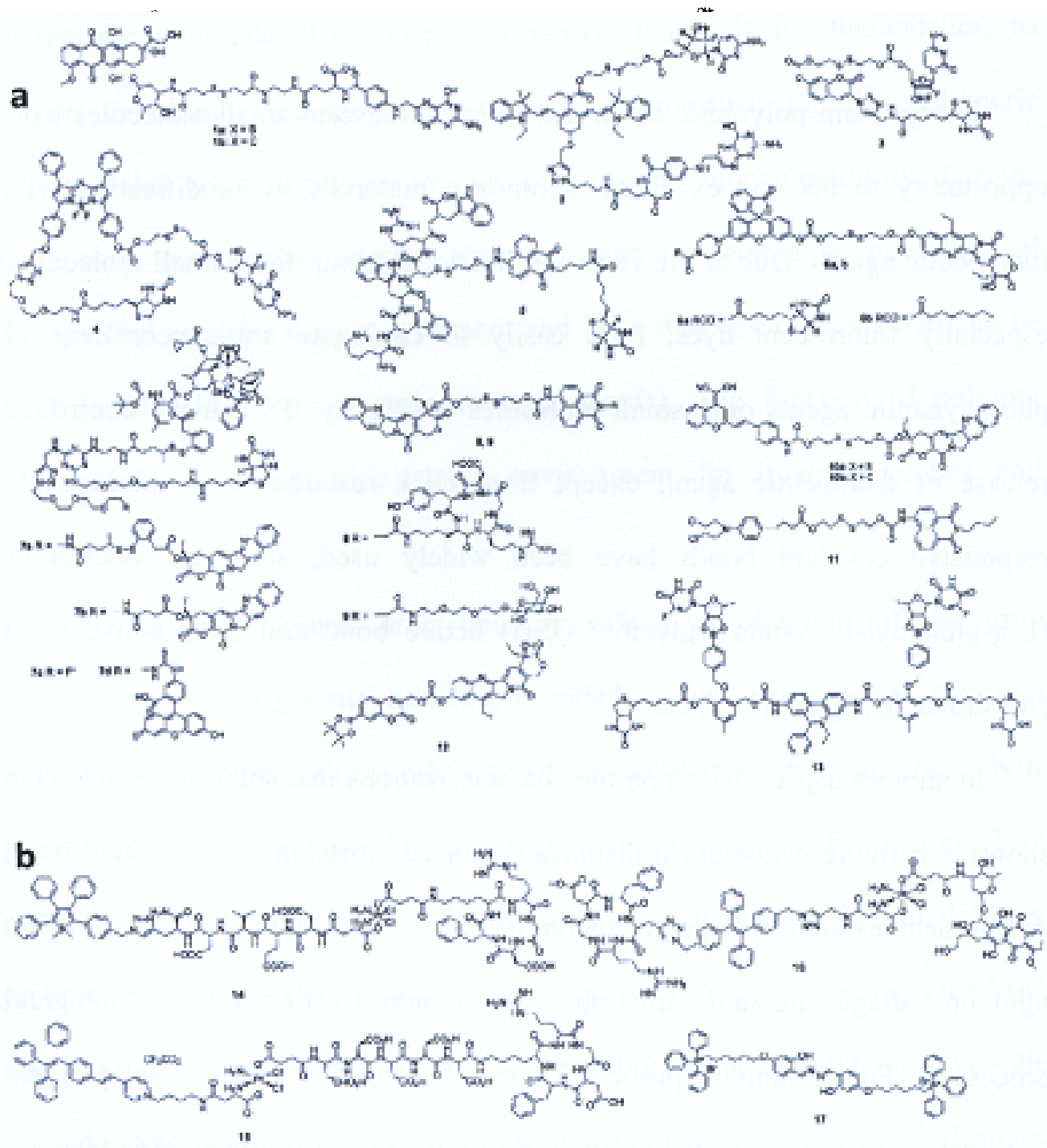

Figure 1-8. GSH and $\mathrm{H} 2 \mathrm{O} 2$ activated theranostic agents (a) and AIE based theranostic 
agents (b).

\subsection{Research motivation and objectives}

\subsubsection{Motivation}

There are some challenges from current fluorescent dyes applied in optical imaging. First, the poor photostability of most of organic dyes limits long-term application, as the fluorescence of these dyes will fade off under strong and longtime irradiation. Second, despite AIE molecules have been reported, but most small molecules still suffer from aggregation caused quenched of fluorescence. As a consequence, the application of fluorescent nanoparticles in bioimaging will be limited due to the aggregated state of fluorescent dyes in nanoparticles. Third, the noisy signal of auto-fluorescence from tissue and the low penetration depth of visible emission wavelength will limit the in vivo applications, which need non-invasive strategies to minimize the damage to body. Thus, this thesis will target these challenging to explore novel fluorescent polymers for future clinical applications.

Morcover, low fluorescence quantum yield of NIR fluorescent dyes caused by non-radiative energy loss, which has potential to generate heat under excited by light resource. This characteristic renders them to be an outstanding photothermal material for cancer treatment. Thus, it is a competitive breakthrough that small molecules are used for photothermal therapy, which will be an alternative candidate to traditional photothermal materials. The 
reason is that most of current photothermal materials are inorganic metal materials, which have concern on poor biodegradability. Finally, the incorporation of imaging strategies and therapeutic materials consisting of polymer nanostructures is evolving concept of theranostic agents.

\subsubsection{Objectives}

In this thesis, my overall objective is to develop a serics of multifunctional polymer nanostructures for cancer imaging and therapy to investigate fluorescent polymers with robust photostability and high photothermal conversion. I also aim to further explore their biomedical applications on bioimaging and cancer therapy. Firstly, we target to design fluorescent polymers with strong photostability and strong fluorescence in aggregated state by using polymer to suppress the intra-molecular aggregation. Second, it is worthy to explore the fluorescent polymers with various emission wavclengths, even could be tuned to NIR region. It is mainly attributed to the reason that NIR fluorescence imaging $(700-1000 \mathrm{~nm})$ is an ideal kind of imaging materials to overcome autofluorescence and penetration concerns. Third, due to the potential ability of converting light energy to heat, polymer nanoparticles with NIR absorption should be studied as photothermal materials. Finally, the multifunctional polymer nanoparticles incorporated with contrast imaging and therapeutic agents should be investigated, due to the ability of imaging and cancer treatment efficacy. We aim to design novel polymeric theranostic materials by perfectly combining the advantages of different imaging and 
therapeutic materials.

\subsection{References}

1. A. D. Bangham, M. M. Standish and J. C. Watkins, J. Mol. Biol., 1965, $13,238-252$.

2. B. S. Pattni, V. V. Chupin and V. P. Torchilin, Chem. Rev., 2015, 115, 10938-10966.

3. T. Safra, F. Muggia, S. Jeffers, D. D. Tsao-Wei, S. Groshen, O. Lyass, R. Henderson, G. Berry and A. Gabizon, Ann. Oncol., 2000, 11, 1029-1033.

4. G. M. Dykes, J. Chem. Technol. Biotechnol., 2001, 76, 903-918.

5. R. P. Brinkhuis, F. P. J. T. Rutjes and J. C. M. van Hest, Polym. Chem., $2011,2,1449-1462$.

6. N. Kamaly, B. Yameen, J. Wu and O. C. Farokhzad, Chem. Rev., 2016, 116, 2602-2663.

7. D. Roy, W. L. A. Brooks and B. S. Sumerlin, Chem. Soc. Rev., 2013, 42, 7214-7243.

8. R. Hoogenboom, H. M. L. Thijs, M. J. H. C. Jochems, B. M. van Lankvelt, M. W. M. Fijten and U. S. Schubert, Chem. Comm., 2008, $5758-5760$

9. B. R. Twaites, C. D. Alarcon, D. Cunliffe, M. Lavigne, S. Pennadam, J. R. Smith, D. C. Gorecki and C. Alexander, J. Control. Release, 2004, 97, 551-566. 
10. H. Wei, S. X. Cheng, X. Z. Zhang and R. X. Zhuo, Prog. Polym. Sci., 2009, 34, 893-910.

11. J. Liu, A. Debuigne, C. Detrembleur and C. Jerome, $A d v$. Healthc. Mater., 2014, 3, 1941-1968.

12. J. Ramos, A. Imaz and J. Forcada, Polym. Chem., 2012, 3, 852-856.

13. F. Meeussen, E. Nies, H. Berghmans, S. Verbrugghe, E. Goethals and F. Du Prez, Polymer, 2000, 41, 8597-8602.

14. H. Ajiro, Y. Takahashi and M. Akashi, Macromolecules, 2012, 45, 2668-2674.

15. S. H. Kim, J. P. K. Tan, K. Fukushima, F. Nederberg, Y. Y. Yang, R. M. Waymouth and J. L. Hedrick, Biomaterials, 2011, 32, 5505-5514.

16. W. J. Zhang, K. Gilstrap, L. Y. Wu, K. C. R. Bahadur, M. A. Moss, Q. A. Wang, X. B. Lu and X. M. He, ACS Nano, 2010, 4, 6747-6759.

17. A. Pitto-Barry and N. P. E. Barry, Polym. Chem., 2014, 5, 3291-3297.

18. H. Z. Deng, J. J. Liu, X. F. Zhao, Y. M. Zhang, J. F. Liu, S. X. Xu, L. D. Deng, A. J. Dong and J. H. Zhang, Biomacromolecules, 2014, 15, 4281-4292.

19. J. J. Chen, J. X. Ding, Y. Zhang, C. S. Xiao, X. L. Zhuang and X. S. Chen, Polym. Chem., 2015, 6, 397-405.

20. J. Hu, J. L. He, M. Z. Zhang and P. H. Ni, Polym. Chem., 2015, 6, 1553-1566.

21. Y. Yu, C. K. Chen, W. C. Law, H. Sun, P. N. Prasad and C. Cheng, Polym. Chem., 2015, 6, 953-961. 
22. M. A. Quadir, S. W. Morton, Z. J. Deng, K. E. Shopsowitz, R. P. Murphy, T. H. Epps and P. T. Hammond, Mol. Pharmaceut., 2014, 11, 2420-2430.

23. B. A. Webb, M. Chimenti, M. P. Jacobson and D. L. Barber, Nat. Rev. Cancer, 2011, 11, 671-677.

24. F. Q. Schafer and G. R. Buettner, Free Radical Bio. Med., 2001, 30, 1191-1212.

25. P. Kuppusamy, H. Q. Li, G. Ilangovan, A. J. Cardounel, J. L. Zweier, K. Yamada, M. C. Krishna and J. B. Mitchell, Cancer Res., 2002, 62, 307-312.

26. M. Huo, J. Yuan, L. Tao and Y. Wei, Polym. Chem., 2014, 5, 1519-1528.

27. E. Fleige, M. A. Quadir and R. Haag, Adv. Drug Deliver. Rev., 2012, 64, 866-884

28. J. Li, M. R. Huo, J. Wang, J. P. Zhou, J. M. Mohammad, Y. L. Zhang, Q. N. Zhu, A. Y. Waddad and Q. Zhang, Biomaterials, 2012, 33, 2310-2320.

29. P. Vader, L. J. van der Aa, J. F. J. Engbersen, G. Storm and R. M. Schiffelers, Pharm. Res., 2011, 28, 1013-1022.

30. H. L. Sun, B. N. Guo, R. Cheng, F. H. Meng, H. Y. Liu and Z. Y. Zhong, Biomaterials, 2009, 30, 6358-6366.

31. K. L. Kozielski, S. Y. Tzeng, B. A. H. De Mendoza and J. J. Green, ACS Nano, 2014, 8, 3232-3241. 
32. L. S. Yan, W. B. Wu, W. Zhao, R. G. Qi, D. M. Cui, Z. G. Xie, Y. B. Huang, T. Tong and X. B. Jing, Polym. Chem., 2012, 3, 2403-2412.

33. Y. L. Colson and M. W. Grinstaff, Adv. Mater., 2012, 24, 3878-3886.

34. D. S. Wilson, G. Dalmasso, L. X. Wang, S. V. Sitaraman, D. Merlin and N. Murthy, Nat. Mater., 2010, 9, 923-928.

35. G. Y. Liu, C. J. Chen, D. D. Li, S. S. Wang and J. Ji, J. Mater. Chem., $2012,22,16865-16871$.

36. C. D. Lux, C. L. McFearin, S. Joshi-Barr, J. Sankaranarayanan, N. Fomina and A. Almutairi, ACS Macro Lett., 2012, 1, 922-926.

37. M. L. Viger, M. Grossman, N. Fomina and A. Almutairi, Adv. Mater., $2013,25,3733-3738$.

38. H. J. Zhang, H. S. Xia, J. Wang and Y. W. Li, J. Control. Release, 2009, 139, 31-39.

39. Y. W. Li, R. Tong, H. S. Xia, H. J. Zhang and J. A. Xuan, Chem. Comm., 2010, 46, 7739-7741.

40. J. Nicolas, S. Mura, D. Brambilla, N. Mackiewicz and P. Couvreur, Chem. Soc. Rev., 2013, 42, 1147-1235.

41. B. D. Ulery, L. S. Nair and C. T. Laurencin, J. Polym. Sci. Part B 2011, $49,832-864$.

42. O. Coulembier, P. Degee, J. L. Hedrick and P. Dubois, Prog. Polym. Sci., 2006, 31, 723-747.

43. C. Wang, Q. Ge, D. Ting, D. Nguyen, H. R. Shen, J. Z. Chen, H. N. Eisen, J. Heller, R. Langer and D. Putnam, Nat. Mater., 2004, 3, 
190-196.

44. D. N. Nguyen, S. S. Raghavan, L. M. Tashima, E. C. Lin, S. J. Fredette, R. S. Langer and C. Wang, Biomaterials, 2008, 29, 2783-2793.

45. P. Wang, J. Hu and P. X. Ma, Biomaterials, 2009, 30, 2735-2740.

46. L. D. Wright, R. T. Young, T. Andric and J. W. Freeman, Biomed. Mater., 2010, 5.

47. G. B. Wei, Q. M. Jin, W. V. Giannobile and P. X. Ma, Biomaterials, 2007, 28, 2087-2096.

48. O. Suwantong, S. Walcetorncheepsawat, N. Sanchavanakit, P. Pavasant, P. Cheepsunthorn, T. Bunaprasert and P. Supaphol, Int. J. Biol Macromol., 2007, 40, 217-223.

49. T. Ahmed, H. Marcal, M. Lawless, N. S. Wanandy, A. Chiu and L. J. R. Foster, Biomacromolecules, 2010, 11, 2707-2715.

50. C. W. Pouton and S. Akhtar, Adv. Drug Deliv. Rev., 1996, 18, 133-162.

51. Y. Ji, X. T. Li and G. Q. Chen, Biomaterials, 2008, 29, 3807-3814.

52. J. Lee, W. Il Choi, G. Tae, Y. H. Kim, S. S. Kang, S. E. Kim, S. H. Kim, Y. Jung and S. H. Kim, Acta Biomater., 2011, 7, 244-257.

53. S. Huang, S. Y. Liu, K. Wang, C. J. Yang, Y. M. Luo, Y. D. Zhang, B. Cao, Y. J. Kang and M. F. Wang, Nanoscale, 2015, 7, 889-895.

54. K. Wang, Y. M. Luo, S. Huang, H. B. Yang, B. Liu and M. F. Wang, J. Polym. Sci. Pol. Chem., 2015, 53, 1032-1042.

55. S. Huang, K. Wang, S. Wang, Y. Wang and M. Wang, Adv. Mater. Inter., 2016, 3, 1600259. 
56. H. J. Diao, K. Wang, H. Y. Long, M. F. Wang and S. Y. Chew, $A d v$. Healthc. Mater., 2016, 5, 529-533.

57. S. He, M. D. Timmer, M. J. Yaszemski, A. W. Yasko, P. S. Engel and A. G. Mikos, Polymer, 2001, 42, 1251-1260.

58. A. Haesslein, H. Ueda, M. C. Hacker, S. Jo, D. M. Ammon, R. N. Borazjani, J. F. Kunzler, J. C. Salamone and A. G. Mikos, J. Control. Release, 2006, 114, 251-260.

59. S. Danti, D. D'Alessandro, A. Pietrabissa, M. Petrini and S. Berrettini, J. Biomed. Mater. Res. A, 2010, 92A, 1343-1356.

60. C. Nguyen, S. Young, J. D. Kretlow, A. G. Mikos and M. Wong, $J$. Oral Maxil. Surg., 2011, 69, 11-18.

61. C. Berkland, E. Pollauf, N. Varde, D. W. Pack and K. Kim, Pharm. Res., $2007,24,1007-1013$.

62. M. Agueros, L. Ruiz-Gaton, C. Vauthier, K. Bouchemal, S. Espuelas, G. Ponchel and J. M. Irache, Eur. J. Pharm. Sci., 2009, 38, 405-413.

63. L. K. Petersen, C. K. Sackett and B. Narasimhan, Acta Biomater., 2010, 6, 3873-3881.

64. S. Samanta, D. R. Bogdanowicz, H. H. Lu and J. T. Koberstein, Macromolecules, 2016, 49, 1858-1864.

65. W. Chen, F. H. Meng, F. Li, S. J. Ji and Z. Y. Zhong, Biomacromolecules, 2009, 10, 1727-1735.

66. W. Chen, F. H. Meng, R. Cheng and Z. Y. Zhong, J. Control. Release, $2010,142,40-46$. 
67. R. Zurita, J. Puiggali and A. Rodriguez-Galan, Macromol. Biosci., 2006, 6, 767-775.

68. P. Uttayarat, A. Perets, M. Y. Li, P. Pimton, S. J. Stachelek, I. Alferiev, R. J. Composto, R. J. Levy and P. I. Lelkes, Acta Biomater., 2010, 6, 4229-4237.

69. J. Poelaert, P. Depuydt, A. De Wolf, S. V. de Velde, I. Herck and S. Blot, J. Thorac. Cardiov. Sur., 2008, 135, 771-776.

70. I. C. Bonzani, R. Adhikari, S. Houshyar, R. Mayadunne, P. Gunatillake and M. M. Stevens, Biomaterials, 2007, 28, 423-433.

71. B. Radeleff, H. Thierjung, U. Stampfl, S. Stampfl, R. Lopez-Benitez, C. Sommer, I. Berger and G. M. Richter, Cardiovasc. Inter. Rad., 2008, 31, 971-980.

72. Q. Li, J. Wang, S. Shahani, D. D. N. Sun, B. Sharma, J. H. Elisseeff and K. W. Leong, Biomaterials, 2006, 27, 1027-1034.

73. Y. C. Wang, X. Q. Liu, T. M. Sun, M. H. Xiong and J. Wang, J. Control. Release, 2008, 128, 32-40.

74. Y. C. Wang, L. Y. Tang, T. M. Sun, C. H. Li, M. H. Xiong and J. Wang, Biomacromolecules, 2008, 9, 388-395.

75. R. Klein and F. R. Wurm, Macromol. Rapid Comm., 2015, 36, 1147-1165.

76. E. M. Pelegri-O'Day, E. W. Lin and H. D. Maynard, J. Am. Chem. Soc., $2014,136,14323-14332$.

77. A. V. Kabanov, E. V. Batrakova and V. Y. Alakhov, J. Controll. 
Release, 2002, 82, 189-212.

78. E. V. Batrakova and A. V. Kabanov, J. Control. Release, 2008, 130, 98-106.

79. M. Y. Kozlov, N. S. Melik-Nubarov, E. V. Batrakova and A. V. Kabanov, Macromolecules, 2000, 33, 3305-3313.

80. Y. Fu and W. J. Kao, Pharm. Res., 2009, 26, 2115-2124.

81. J. A. DiRamio, W. S. Kisaalita, G. F. Majetich and J. M. Shimkus, Biotechnol. Progr., 2005, 21, 1281-1288.

82. A. N. Buxton, J. Zhu, R. Marchant, J. L. West, J. U. Yoo and B. Johnstone, Tissue Eng., 2007, 13, 2549-2560.

83. M. O'brien, A. Sandler, A. Popovich, H. Ganchev, N. Bogdanova and J. Miziara, Lung Cancer-J Iaslc, 2005, 49, S37-S37.

84. R. N. Chen, G. M. Wang, C. H. Chen, H. O. Ho and M. T. Sheu, Biomacromolecules, 2006, 7, 1058-1064.

85. J. L. Ifkovits, E. Tous, M. Minakawa, M. Morita, J. D. Robb, K. J. Koomalsingh, J. H. Gorman, R. C. Gorman and J. A. Burdick, P. Natl. Acad Sci USA, 2010, 107, 11507-11512.

86. S. Coca, H. J. Paik and K. Matyjaszewski, Macromolecules, 1997, 30, $6513-6516$

87. J. Aimi, L. A. McCullough and K. Matyjaszewski, Macromolecules, $2008,41,9522-9524$.

88. K. Matyjaszewski, Abstr. Pap. Am. Chem. S., $2011,241$.

89. H. Shinoda and K. Matyjaszewski, Macromolecules, 2001, 34, 
6243-6248.

90. D. J. Keddie, Chem. Soc. Rev., 2014, 43, 496-505.

91. G. Moad, Acs Sym. Ser., 2015, 1187, 211-246.

92. R. B. Grubbs, Polym. Rev., 2011, 51, 104-137.

93. G. Delaittre, J. Rieger and B. Charleux, Macromolecules, 2011, 44, $462-470$.

94. W. H. Binder and R. Sachsenhofer, Macromol. Rapid Comm., 2008, 29, 952-981.

95. B. Le Droumaguet and K. Velonia, Macromol Rapid Comm, 2008, 29, 1073-1089.

96. A. J. Qin, J. W. Y. Lam and B. Z. Tang, Macromolecules, 2010, 43, 8693-8702.

97. A. J. Qin, J. W. Y. Lam and B. Z. Tang, Chem. Soc. Rev., 2010, 39, $2522-2544$.

98. Y. R. Zhang, G. Chen, Y. L. Lin, L. F. Zhao, W. Z. Yuan, P. Lu, C. K. W. Jim, Y. M. Zhang and B. Z. Tang, Polym. Chem., 2015, 6, 97-105.

99. P. P. Datta and M. K. Kiesewetter, Macromolecules, 2016, 49, 774-780.

100. N. E. Kamber, W. Jeong, R. M. Waymouth, R. C. Pratt, B. G. G. Lohmeijer and J. L. Hedrick, Chem. Rev., 2007, 107, 5813-5840.

101. L. Zhang, F. Nederberg, J. M. Messman, R. C. Pratt, J. L. Hedrick and C. G. Wade, J. Am. Chem. Soc., 2007, 129, 12610.

102. J. L. Hedrick, M. Trollsas, C. J. Hawker, B. Atthoff, H. Claesson, A. Heise, R. D. Miller, D. Mecerreyes, R. Jerome and P. Dubois, 
Macromolecules, 1998, 31, 8691-8705.

103. R. M. Mainardes and R. C. Evangelista, J Microencapsul, 2005, 22, $13-24$.

104. J. Vandorpe, E. Schacht, S. Stolnik, M. C. Garnett, M. C. Davies, L. Illum and S. S. Davis, Biotechnol. Bioeng., 1996, 52, 89-95.

105. C. X. Song, V. Labhasetwar, H. Murphy, X. Qu, W. R. Humphrey, R. J. Shebuski and R. J. Levy, J. Control. Release, 1997, 43, 197-212.

106. D. Lemoine and V. Preat, J. Control. Release, 1998, 54, 15-27.

107. M. F. Zambaux, F. Bonneaux, R. Gref, P. Maincent, E. Dellacherie, M. J. Alonso, P. Labrude and C. Vigneron, J. Control. Release, 1998, 50, $31-40$.

108. Y. Mai and A. Eisenberg, Chem. Soc. Rev., 2012, 41, 5969-5985.

109. M. S. Bakshi, A. Kaura, P. Bhandari, G. Kaur, K. Torigoe and K. Esumi, J. Nanosci. Nanotechno., 2006, 6, 1405-1410.

110. S. S. Cai, K. Vijayan, D. Cheng, E. M. Lima and D. E. Discher, Pharm Res, 2007, 24, 2099-2109.

111. Y. Geng, P. Dalhaimer, S. S. Cai, R. Tsai, M. Tewari, T. Minko and D. E. Discher, Nat. Nanotechnol., 2007, 2, 249-255.

112. G. Srinivas, D. E. Discher and M. L. Klein, Nat. Mater, 2004, 3, 638-644.

113. Q. H. Sun, X. R. Sun, X. P. Ma, Z. X. Zhou, E. L. Jin, B. Zhang, Y. Q. Shen, E. A. Van Kirk, W. J. Murdoch, J. R. Lott, T. P. Lodge, M. Radosz and Y. L. Zhao, Adv. Mater., 2014, 26, 7615-7621. 
114. Q. H. Sun, X. P. Ma, B. Zhang, Z. X. Zhou, E. L. Jin, Y. Q. Shen, E. A. Van Kirk, W. J. Murdoch, M. Radosz and W. L. Sun, Biomater. Sci-Uk, $2016,4,958-969$

115. W. B. Liechty, D. R. Kryscio, B. V. Slaughter and N. A. Peppas, Annu. Rev. Chem. Biomol., 2010, 1, 149-173.

116. L. Cheng, K. Yang, Q. Chen and Z. Liu, ACS Nano, 2012, 6, $5605-5613$.

117. J. L. Geng, K. Li, K. Y. Pu, D. Ding and B. Liu, Small, 2012, 8, 2421-2429.

118. C. Wang, H. Xu, C. Liang, Y. M. Liu, Z. W. Li, G. B. Yang, H. Cheng, Y. G. Li and Z. Liu, ACS Nano, 2013, 7, 6782-6795.

119. L. Q. Xiong, A. J. Shuhendler and J. H. Rao, Nat. Commun., 2012, 3.

120. L. G. Xu, L. Cheng, C. Wang, R. Peng and Z. Liu, Polym. Chem., 2014, 5, 1573-1580

121. J. Yang, J. Choi, D. Bang, E. Kim, E. K. Lim, H. Park, J. S. Suh, K. Lee, K. H. Yoo, E. K. Kim, Y. M. Huh and S. Haam, Angew. Chem. Int. Ed., $2011, \mathbf{5 0}, 441-444$

122. K. Yang, H. Xu, L. Cheng, C. Y. Sun, J. Wang and Z. Liu, Adv. Mater., $2012,24,5586-5592$.

123. Z. B. Zha, X. L. Yue, Q. S. Ren and Z. F. Dai, Adv. Mater., 2013, 25, $777-782$

124. L. Fass, Mol. Oncol, , 2008, 2, 115-152.

125. J. K. Willmann, N. van Bruggen, L. M. Dinkelborg and S. S. Gambhir, 
Nat. Rev. Drug Discov., 2008, 7, 591-607.

126. C. Wu, C. Szymanski and J. McNeill, Langmuir : the ACS journal of surfaces and colloids, 2006, 22, 2956-2960.

127. B. Sun, B. Zhao, D. D. Wang, Y. B. Wang, Q. Tang, S. J. Zhu, B. Yang and H. C. Sun, Nanoscale, 2016, 8, 9837-9841.

128. W. Zhang, H. Sun, S. Y. Yin, J. J. Chang, Y. H. Li, X. Y. Guo and Z. Yuan, J. Mater. Sci, 2015, 50, 5571-5577.

129. J. Pennakalathil, A. Ozgun, I. Durmaz, R. Cetin-Atalay and D. Tuncel, J. Polym. Sci. A Polym. Chem., 2015, 53, 114-122.

130. Z. M. Hudson, D. J. Lunn, M. A. Winnik and I. Manners, Nat. Commun., $2014,5$.

131. C. Pietsch, R. Hoogenboom and U. S. Schubert, Angew. Chem. Int. Ed., 2009, 48, 5653-5656.

132. X. Wu, G. J. Price and R. H. Guy, Mol. Pharmaceut., 2009, 6, 1441-1448.

133. L. Ionov, V. Bocharova and S. Diez, Soft Matter, 2009, 5, 67-71.

134. S. Berger, A. Synytska, L. Ionov, K. J. Eichhorn and M. Stamm, Macromolecules, 2008, 41, 9669-9676.

135. L. De Jong, X. Moreau, A. Thiery, G. Godeau, M. W. Grinstaff and P. Barthelemy, Bioconjugate Chem., 2008, 19, 891-898.

136. C. H. Li, Y. X. Zhang, J. M. Hu, J. J. Cheng and S. Y. Liu, Angew. Chem. Int. Ed., 2010, 49, 5120-5124.

137. A. M. Breul, M. D. Hager and U. S. Schubert, Chem. Soc. Rev., 2013, 
42, 5366-5407.

138. R. R. Hu, Y. Kang and B. Z. Tang, Polym. J., 2016, 48, 359-370.

139. Y. Liu, S. M. Chen, J. W. Y. Lam, P. Lu, R. T. K. Kwok, F. Mahtab, H. S. Kwok and B. Z. Tang, Chem. Mater., 2011, 23, 2536-2544.

140. J. Wang, J. Mei, W. Z. Yuan, P. Lu, A. J. Qin, J. Z. Sun, Y. G. Ma and B. Z. Tang, J. Mater. Chem., 2011, 21, 4056-4059.

141. Y. N. Hong, J. W. Y. Lam and B. Z. Tang, Chem. Soc. Rev., 2011, 40, $5361-5388$.

142. Y. N. Hong, J. W. Y. Lam, S. J. Chen and B. Z. Tang, Aust. J. Chem., $2011,64,1201-1208$.

143. F. Mahtab, J. W. Y. Lam, Y. Yu, J. Z. Liu, W. Z. Yuan, P. Lu and B. Z. Tang, Small, 2011, 7, 1448-1455.

144. G. J. Wang, R. C. Zhang, C. Xu, R. Y. Zhou, J. Dong, H. T. Bai and X. W. Zhan, ACS Appl. Mater. Inter., 2014, 6, 11136-11141.

145. Y. M. Li, H. S. Yu, Y. F. Qian, J. M. Hu and S. Y. Liu, Adv. Mater., $2014,26,6734-6741$.

146. C. P. Ma, X. Q. Zhang, K. Wang, X. Y. Zhang, Y. H. Zhou, H. L. Liu and Y. Wei, Polym. Chem., 2015, 6, 3634-3640.

147. Y. Y. Bao, H. De Keersmaecker, S. Corneillic, F. Yu, H. Mizuno, G. F. Zhang, J. Hofkens, B. Mendrek, A. Kowalczuk and M. Smet, Chem. Mater., 2015, 27, 3450-3455.

148. S. Santra, C. Kaittanis and J. M. Perez, Mol. Pharmaceut., 2010, 7, 1209-1222. 
149. Z. Y. Liao, H. J. Wang, X. D. Wang, P. Q. Zhao, S. Wang, W. Y. Su and J. Chang, Adv. Funct. Mater., 2011, 21, 1179-1186.

150. S. M. Lec, Y. Song, B. J. Hong, K. W. MacRenaris, D. J. Mastarone, T. V. O'Halloran, T. J. Meade and S. T. Nguyen, Angew. Chem. Int. Ed., $2010,49,9960-9964$.

151. J. Zhang, H. Y. Chen, L. Xu and Y. Q. Gu, J. Controlled. Release, 2008, $131,34-40$.

152. X. H. Zheng, D. Xing, F. F. Zhou, B. Y. Wu and W. R. Chen, Mol. Pharmaceut., 2011, 8, 447-456.

153. S. Bhuniya, S. Maiti, E. J. Kim, H. Lee, J. L. Sessler, K. S. Hong and J. S. Kim, Angew. Chem. Int. Ed., 2014, 53, 4469-4474.

154. T. Kim, H. M. Jeon, H. T. Le, T. W. Kim, C. Kang and J. S. Kim, Chem. Comm., 2014, 50, 7690-7693.

155. S. Maiti, N. Park, J. H. Han, H. M. Jeon, J. H. Lee, S. Bhuniya, C. Kang and J. S. Kim, J. Am. Chem. Soc, 2013, 135, 4567-4572.

156. M. H. Lee, J. Y. Kim, J. H. Han, S. Bhuniya, J. L. Sessler, C. Kang and J. S. Kim, J. Am. Chem. Soc, 2012, 134, 12668-12674.

157. X. M. Wu, X. R. Sun, Z. Q. Guo, J. B. Tang, Y. Q. Shen, T. D. James, H. Tian and W. H. Zhu, J. Am. Chem. Soc, 2014, 136, 3579-3588.

158. E. J. Kim, S. Bhuniya, H. Lec, H. M. Kim, C. Cheong, S. Maiti, K. S. Hong and J. S. Kim, J. Am. Chem. Soc, 2014, 136, 13888-13894.

159. Y. Y. Yuan, R. T. K. Kwok, R. Y. Zhang, B. Z. Tang and B. Liu, Chem. Comm., 2014, 50, 11465-11468. 
160. Y. Y. Yuan, R. T. K. Kwok, B. Z. Tang and B. Liu, J. Am. Chem. Soc, 2014, 136, 2546-2554.

161. Y. Y. Yuan, Y. L. Chen, B. Z. Tang and B. Liu, Chem. Comm., 2014, 50, 3868-3870.

162. Q. L. Hu, M. Gao, G. X. Feng and B. Liu, Angew. Chem. Int. Ed., 2014, 53, 14225-14229.

163. S. Kerkhofs, T. Willhammar, H. V. D. Noortgate, C. E.A. Kirschhock, E. Breynaert, G. V. Tendeloo, S. Bals and J. A. Materns, Chem. Mater, $2015,27,5161-5169$

164. R. Tanbour, A.M. Martins, W.G.Pitt, and G. A. Husseini, Curr. Pharm. Des., 2016, 22, 2796-2807

165. C. Poetsch, U. S. Schubert and R. Hoogenboom, Chem. Comm., 2011, 47, $8750-8765$

166. Kin, J, -H, Jung, Y., Lee,D. and Jang,W. -D., Adv. Mater, 2016, 28, 3499-3503

167. Lyu, S., Untereker, D. Int. J. Mol. Sci. 2009, 10, 4033-4065

168. Allcock, H. R.; Lampe, F. W. Chontemporary Polymer Chemistry; Prentice-Hall: Englewood Cliffs, NJ, 1981

169. Y. Murakami, Y. Tabata and Y. Ikada, Drug Delivery, 1996, 4, 231-238

170. H. Maeda, J. Wu, T. Sawa, Y. Matsumura, K. Hori, J. Controlled Release, 2000, 65, 271-284

171. N. S. Abadeer, C. J. Murphy, J. Phys. Chem. C., 2016, 120, 4691-4716

172. J. M. Tan, J. O.Tan, A. Murthy,D. R. Ingram, L. L. Ma, K. Travis, K. P. 
Johnston, K. V. Sokolov, ACS Nano, 2010, 4, 2178-2184

173. M. Elsabahy, G. S. Heo, S-M. Lim, G. Sun, K. L. Wooley, Chem. Rev., $2015, \mathbf{1 1 5}, 10967-11011$

174. H. Lusic, M. W. Grinstaff, Chem. Rev., 2013, 113, 1641-1666

175. N. Savage, Nature, 2013, 502, S90-S91

176. E. Philips, O. Penate-Medina, P. B. Zanzonico, R. D. Carvajal, P. Mohan, Y. Ye, J. Humm, M. Gogen, H. Kalaigian, H. Schoder, et al. Sci. Transl. Med., 2014, 6, 260ra149

177. K. Matyjaszewski, Macromolecules, 2012, 45, 4015-4039

178. P. B. Zetterlund, S. C. Thickett, S. Perrier, E. B. Lami, M. Lansalot, Chem. Rev., 2015, 115, 9745-9800 


\section{Chapter 2 Highly fluorescent and bioresorbable}

\section{polymeric nanoparticles with enhanced}

\section{photostability for cell imaging}

In this chapter, I present a facile and novel strategy of enhancing the photostability of organic fluorophores for bioimaging applications. As a proof of concept, bright and robust fluorescence was observed in solid states of a well-defined synthetic polymer polycaprolactone consisting of di(thiophene-2-yl)-diketopyrrolopyrrole covalently linked in the middle of the polymer chain as a biocompatible and bioresorbable matrix. The nanoparticles prepared through a nanoprecipitation process of these polymers could be internalized by both tumor cells and stem cells with little cytotoxicity. Moreover, these highly fluorescent nanoparticles exhibited significantly enhanced photostability compared to commercial quantum dots or physical blends of dye/polymer complex in cell imaging and long-term tracing. This work has been published in Nanoscle in 2015.

\subsection{Introduction}

Fluorescence imaging, due to its high specificity, sensitivity and imaging contrast, has proven to be an indispensable tool in modern biotechnology for advancing our understanding of various biological 
systems. ${ }^{1-3}$ Among a varicty of probes for fluorescence imaging, small organic fluorophores have been most widely used owing to their high fluorescence quantum yield, relatively low toxicity, and structural versatility that enables facile tuning of the optical properties and conjugation with biomolecules. ${ }^{3-7}$ For example, Alexa Fluor ${ }^{B}$ family of fluorescent molecules has been commercially available and used as cell and tissue labels in fluorescence microscopy and cell biology. Another example is $4^{\prime}, 6$-diamidino-2-phenylindole, denoted as DAPI, which has been a commonly used fluorescent probe for selectively labeling of DNA in cell nucleus.

Despite these advantages, most organic fluorophores suffer from their poor photostability, i.e. the fluorescence intensity decays quickly (tens of seconds) under continuous exposure to light. This phenomenon, also called photobleaching, limits the ability of employing fluorescence microscopy or spectroscopy for long-term tracking of biological activities. Previous studies indicate that the formation of non-fluorescent triplet states (denoted as $T_{1}$ ), following the photonic excitation process, and the presence of molecular oxygen play a major role in the photobleaching of organic fluorophores. In air-saturated solutions, reactions between molecular oxygen and $T_{1}$ lead to substantial generation of reactive oxygen specics (ROS), including $\mathrm{HO} \bullet, \mathrm{HO}_{2} \bullet$, and $\mathrm{H}_{2} \mathrm{O}_{2}$, and rapid photobleaching.

Several strategies such as oxygen depletion and addition of protective agents as triplet-state quenchers, although being able to reduce photobleaching to some 
extent, have achieved limited success in fluorescence-based bioimaging." Recently, Blanchard and coworkers ${ }^{8}$ have reported a strategy of covalently linking protective moicties (e.g. cyclooctatetraene, 4-nitrobenzyl alcohol, 6-hydroxy-2,5,7,8-tetramethylchroman-2-carboxylic acid) to cyanine fluorophores to enhance the photostability. Single-molecule fluorescence imaging showed remarkable enhancement of photostability in these modified fluorophores compared to the native ones without protective agents. Nevertheless, the improvement of photostability varies with different members of the cyanine class, suggesting the need for a deeper understanding of how the protective agents provide photostabilization. ${ }^{8}$ Relatively high photostability has been observed in inorganic semiconductor quantum dots, ${ }^{9-13}$ organic dye-silica core/shell NPs, ${ }^{14,15}$ some organic and polymeric fluorescent dots, ${ }^{16,17}$ and some optimized fluorescence proteins through mutagenesis. ${ }^{18}$ For example, enhanced photostability has been recently reported in nanoparticles composed of organic fluorophores with aggregation induced emission (AIE) effect, ${ }^{16,19-21}$ but such improvement has been only observed in limited groups of organic dyes and polymers. Despite these recent advances, a general strategy of obtaining highly luminescent, photostable and non-phototoxic fluorophores for long-term bioimaging is still in great demand. ${ }^{3}$

In this chapter, we will explore a facile strategy of enhancing the photostability by covalently linking an organic fluorophore, di(thiophene-2-yl)-diketopyrrolopyrrole (DPP), in the middle of a biocompatible and bioresorbable polymer chain, polycaprolactone (PCL) 
(Figure. 2-1). This polymer, denoted as PCL-DPP-PCL, has a number-average molecular weight $\left(M_{\mathrm{n}}\right)$ of $38,300 \mathrm{Da}$ and a polydispersity index $\left(M_{\mathrm{w}} / M_{\mathrm{n}}\right)$ of 1.98 . In contrast to small-molecule DPP derivatives which often show dramatic fluorescence quenching in solid states due to significant intermolecular $\pi-\pi$ aggregation, we found that PCL-DPP-PCL tethered with long PCL chains shows remarkably bright fluorescence in solid states, suggesting that the aggregation of DPP moieties is suppressed by the covalently attached PCL chains. In addition, PCL as a biocompatible and bioresorbable polyester has attracted much attention for use as an implantable biomaterial in tissue enginecring and drug delivery. ${ }^{22}$ Biodegradation of PCL is slow compared to other polymers, thus making it suitable for long-term delivery extending over a period of more than one year.

We expected that the integration of strong fluorescence of DPP with the biophysical properties of PCL could make PCL-DPP-PCL an ideal fluorescence probe for bioimaging. Considering the hydrophobic nature of both DPP and PCL in this polymer, we used Pluronic ${ }^{\circledR} 127$ as a surfactant and followed a method of nanoprecipitation to disperse PCL-DPP-PCL as nanoparticles (NPs) in aqueous media (Figure. 2-1). The resulting NPs were highly fluorescent and showed remarkably enhanced photostability compared to commercial organic fluorophores in imaging of both human liver cancer cell line (HuH-7) and porcine mescnchymal stem cells (MSCs). Moreover, these polymeric NPs showed brighter and more robust fluorescence properties compared to 
commercially available imaging probes such as quantum dots $\left(\right.$ Qtracker $\left.^{\circledR}\right)$ in long-term tracing of cells.

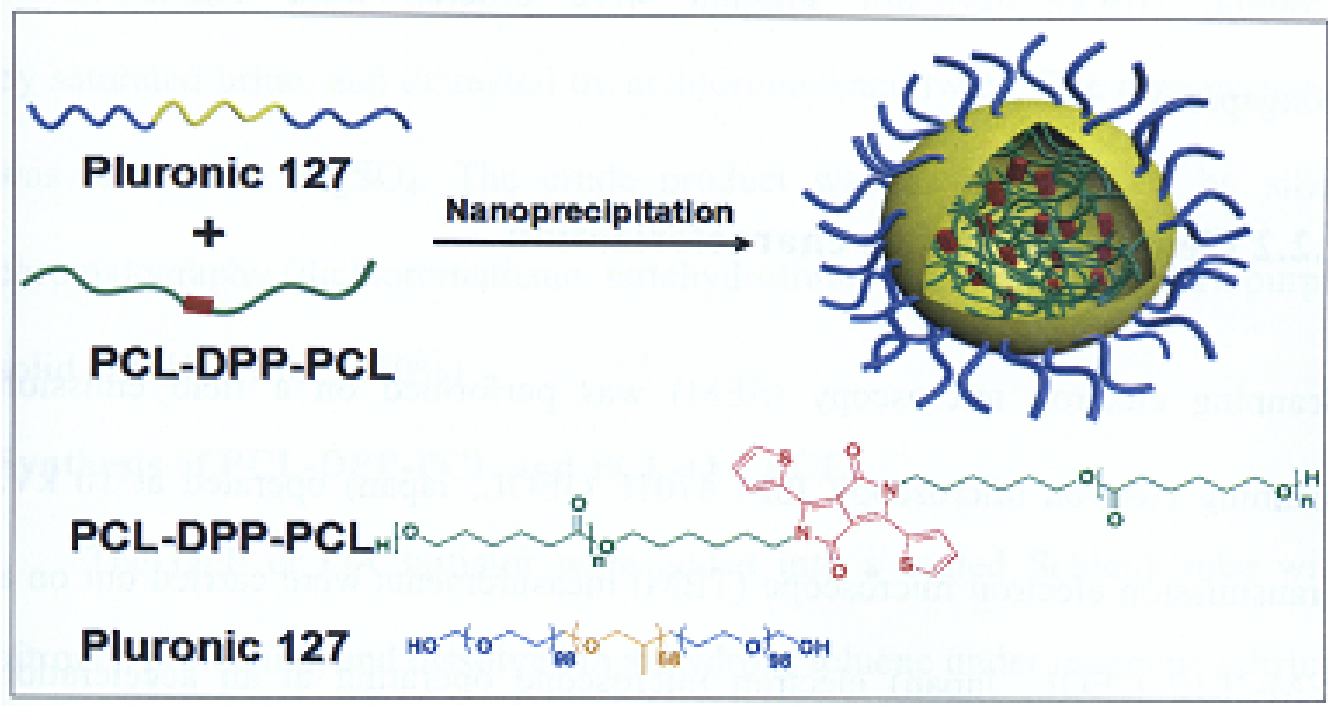

Figure. 2-1 Schematic illustration of the preparation of PCL-DPP-PCL nanoparticles via nanoprecipitation process in the presence of Pluronic 127 as the stabilizer.

\subsection{Materials and Methods.}

\subsubsection{Materials and agents}

All chemical reagents were obtained from commercial suppliers and used without any further purification. Dulbecco's Modified Eagle's Medium (DMEM), fetal bovine serum (FBS), penicillin /streptomycin mixture, phosphate buffered saline (PBS), Alexa Fluor $^{\circledR} 633$ phalloidin, TrypLETM Express Enzyme (1×), Qtracker ${ }^{\circledR} 705$ Cell Labeling Kit and PrestoBlue cell 
viability reagent were purchased from Life Technologies (Singapore). Other reagents including 4', 6-diamidino-2-phenylindole (DAPI), Bovine Serum Albumin (BSA), formalin solution were ordered from Sigma-Aldrich (Singapore)

\subsubsection{General materials characterization}

Scanning electron microscopy (SEM) was performed on a field emission scanning electron microscopy JSM $6701 \mathrm{~F}$ (JEOL, Japan) operated at $10 \mathrm{kV}$. Transmission electron microscope (TEM) measurements were carried out on a JEM-3010 (JEOL, Japan) electron microscope operating at an acceleration voltage of $120 \mathrm{kV}$. UV-vis transmission spectrum was recorded on a UV-2450 (SHIMADZU, Japan) spectrophotometer. Photoluminescence spectra were recorded with LS 55 (PerkinElmer, USA) fluorescence spectrometer. The particle size was measured with a dynamic light scattering (DLS) instrument, Zetasizer Nano ZS (Malvern, UK).

\subsubsection{The preparation of PCL-DPP-PCL nanoparicles}

2,5-bis(6-hydroxyhexyl)-3,6-di(thiophen-2-yl)pyrrolo[3,4-c/pyrrole-1,4(2H,5H )-dione (DPPHT). Potassium carbonate $(8.28 \mathrm{~g}, 60 \mathrm{mmol})$ and DPP-monomers (3,6-di(thiophen-2-yl)pyrrolo[3,4-c]pyrrole-1,4(2H,5H)-dione) $(6 \mathrm{~g}, 20 \mathrm{mmol})$ were mixed in $200 \mathrm{~mL}$ of dimethylformamide under stirring in a $250 \mathrm{~mL}$ three-neck round bottom flask under nitrogen protection. The mixture was heated up to $140{ }^{\circ} \mathrm{C}$ followed by addition of 6-bromo-1-hexanol (10.88 g, 60 
mmol) dissolved in $25 \mathrm{~mL}$ of dimethylformamide dropwise over 1 hour. The reaction mixture was stirred at $140{ }^{\circ} \mathrm{C}$ overnight. Then the flask was allowed to cool to room temperature and rinsed with chloroform. The product was washed by saturated brine, and extracted by dichloromethane twice. The organic phase was dried with $\mathrm{MgSO}_{4}$. The crude product was further purified by silica chromatography (dichloromethane: tertahydrofuran $=1: 1$ ) to obtain dark purple solid DPPHT (1.02 g, 10\%)

\section{Synthesis of PCL-DPP-PCL and PCL-QA-PCL.}

The DPP or QA initiator were added into a sealed Schlenk tube with nitrogen protection, and dissolved in anhydrous toluene under magnetic stirring. Stannous octoate as the catalyst was injected through the rubber seal with a syringe. $\varepsilon$-Caprolactone was injected into the reaction mixture. The mixture was degassed with $\mathrm{N}_{2}$-bubbling before the Schlenk tube was submerged in an oil bath and the reaction mixture was heated up to $110^{\circ} \mathrm{C}$ and kept at this temperature overnight. Crude product was purified by dissolved in tetrahydrofuran and precipitated in cold methanol twice.

General procedure for the synthesis of the PCL-DPP-PCL nanoparticles. In a typical procedure, $6 \mathrm{~mL}$ of PCL-DPP-PCL $(3 \mathrm{mg} / \mathrm{mL}$ in THF) was injected into $30 \mathrm{~mL}$ of vigorously stirred solution of Pluronic ${ }^{\circledR} 127(8.3 \mathrm{~g} / \mathrm{L}$ in water) at room temperature. After being stirred for 3 mins, the sample was left for stabilization for about 2 hours. Then the sample was subjected to dialysis against di-water using a $4 \mathrm{kDa}$ membrane. The resulting NPs were separated by centrifugation at $11,500 \mathrm{~g}$ for 15 mins, and the sediments were redispersed in 
di-water before characterization or dispersed in $1 \times$ PBS buffer for cell experiments.

\subsubsection{Procedures of cell experiments}

\section{Cell culture}

HuH-7 Cell Line (Hepato cellular carcinoma cells) were obtained from National Cancer Center (Singapore) and cultured in Dulbecco's Modified Eagle's Medium (DMEM) supplemented with $10 \%$ (vol/vol) fetal bovine serum (FBS) and $1 \%$ penicillin /streptomycin mixture at $37{ }^{\circ} \mathrm{C}$ with $5 \% \quad \mathrm{CO}_{2}$. Mesenchymal stem cells (MSCs) were isolated from porcine bone marrow aspirates following institutional guidelines, which demonstrated good purity and stability ${ }^{33}$. MSCs were also cultured in DMEM with $10 \%$ FBS and a penicillin $(100 \mathrm{U} / \mathrm{ml}) /$ streptomycin $(100 \mu \mathrm{g} / \mathrm{mL})$ mixture at $37{ }^{\circ} \mathrm{C}$ with $5 \%$ $\mathrm{CO}_{2}$.

\section{In vitro cellular uptake}

In order to explore the cellular uptake of PCL-DPP-PCL NPs and physically blended PCL/DPP NPs, HuH-7 or MSCs were seeded on Petri dish and cultured in $37^{\circ} \mathrm{C}$ with $5 \% \mathrm{CO}_{2}$. After $12 \mathrm{~h}$ for attachment, NPs were added to the medium $(0.2 \mathrm{mg} / \mathrm{mL})$ and incubated with cells at $37{ }^{\circ} \mathrm{C}$ with $5 \% \mathrm{CO}_{2}$. After $24 \mathrm{~h}$, excess NPs were washed with $1 \times$ PBS for three times. After that, cells were fixed by formalin solution for $20 \mathrm{~min}$ and then washed by $1 \times \mathrm{PBS}$ extensively for three times. Then cells were permeabilized with $0.1 \%(\mathrm{vol} / \mathrm{vol})$ Triton $\mathrm{X}-100$ in $1 \times \mathrm{PBS}$ for 5 minutes at room temperature. After washing twice 
by $1 \times$ PBS, cells were blocked for $30 \mathrm{~min}$ in $1 \times \mathrm{PBS}$ containing $1 \%(\mathrm{wt} / \mathrm{vol})$ BSA. Then Alexa Fluor $^{\mathbb{B}} 633$ phalloidin in $1 \times$ PBS was added to stain filamentous actin (F-actin) cytoskeleton for $1 \mathrm{~h}$ at room temperature. After washing three times, nucleus was stained by DAPI for $1 \mathrm{~min}$ at room temperature. Then samples were washed three times and then added in fresh $1 \times$ PBS. Lasers of 405,561 , and $633 \mathrm{~nm}$ were used to excite DAPI, NPs, and Alexa Fluor ${ }^{\circledR} 633$ phalloidin, respectively. In order to observe cellular uptake of PCL-QA-PCL NPs, similar procedures were followed except that the lasers of 405,488 , and $633 \mathrm{~nm}$ were used to excite DAPI, PCL-QA-PCL NPs, and Alexa Fluor $^{\circledR} 633$ phalloidin, respectively. The corresponding fluorescence emissions were recorded through a confocal microscope (LSM 780, Carl Zciss, Gcrmany) using a band-pass filter combination including $421-481 \mathrm{~nm}, 569-621 \mathrm{~nm}$, and 650-758 $\mathrm{nm}$ for imaging in three individual channels (Objective: LD Plan-Neofluar 20x/0.4 Korr M27).

\section{Photobleach assay}

In order to perform the photobleach test of PCL-DPP-PCL NPs and physically blended PCL/DPP NPs, HuH-7 or MSCs were seeded on a Petri dish and cultured in $37^{\circ} \mathrm{C}$ with $5 \% \mathrm{CO}_{2}$. After $12 \mathrm{~h}$ for attachment, NPs were added to the medium and incubated with cells at $37{ }^{\circ} \mathrm{C}$ with $5 \% \mathrm{CO}_{2}$. After $24 \mathrm{~h}$, excess NPs were washed with $1 \times$ PBS for three times. After that, cells werc fixed by formalin solution for $20 \mathrm{~min}$ and then washed by $1 \times$ PBS extensively for three times. Then cells were permeabilized with $0.1 \%$ (vol/vol) Triton $\mathrm{X}-100$ in $1 \times \mathrm{PBS}$ for 5 minutes at room temperature. After washing twice by 
$1 \times \mathrm{PBS}$, cells were blocked for $30 \mathrm{~min}$ in $1 \times \mathrm{PBS}$ containing $1 \%(\mathrm{wt} / \mathrm{vol}) \mathrm{BSA}$. Then Alexa Fluor $^{\circledR} 633$ phalloidin in $1 \times$ PBS was added to stain filamentous actin ( $\mathrm{F}$-actin) cytoskeleton for $1 \mathrm{~h}$ at room temperature. After washing three times, nucleus was stained by DAPI for $1 \mathrm{~min}$ at room temperature. Then samples were washed three times and then added in fresh $1 \times$ PBS. After being multiply-stained with a cocktail of three dyes, both MSCs and HuH-7 were continuously exposed to $405 \mathrm{~nm}, 561 \mathrm{~nm}$, and $633 \mathrm{~nm}$ laser irradiation with a higher intensity than that in cellular uptake assays and the fluorescence emissions from each dye were recorded at specific time points (Objective: LD Plan-Neofluar 20x/0.4 Korr M27). The nominal laser powers were calculated by multiplying the maximum laser power by the percentage of intensity output during photobleaching. Images were obtained in every scans and bleaches were repeated after every scans. The time in second indicated on images was the moments for scanning.

\section{Long term cell tracing}

HuH-7 tumor cells and MSC were seeded in a 6-well plate and cultured at $37{ }^{\circ} \mathrm{C}$ with $5 \% \mathrm{CO}_{2}$. After $12 \mathrm{~h}$ for attachment, $2 \mathrm{nM}$ PCL-DPP-PCL NPs and Qtracker $^{\circledR} 705$ were added, respectively. After incubation for $48 \mathrm{~h}$, the culture medium was removed and cells were washed three times by $1 \times$ PBS to remove excess NPs. Then the cells were detached by TrypLE Express and subcultured into another 6-well plate. After a number of days (Day 1, 3, 5, 7 for HuH-7 and Day 1, 7, 14 for MSC), cells were imaged by fluorescent microscopy (Olympus IX71, excited at 530-550 nm, emission filter 575IF). Then the cells were 
washed with $1 \times$ PBS and detached by TrypLE Express. After centrifugation (500 g, $5 \mathrm{~min}$ ), supernatant were discarded and cell pellets were collected and resuspended in $1 \times$ PBS for flow cytometry (LSRII, BD Biosciences, Singapore). The fluorescence of PCL-DPP-PCL NPs and Qtracker ${ }^{(B)} 705$ were detected using PE (excitation: $561 \mathrm{~nm}$, emission: $567 \mathrm{~nm}-597 \mathrm{~nm}$ ) and PE-cy5 (excitation: $561 \mathrm{~nm}$, emission: $660 \mathrm{~nm}-760 \mathrm{~nm}$ ) channels, respectively. The cells without any fluorescence labcling were used as control. The flow cytometry data were analyzed using FlowJo software.

\section{In vitro cytotoxicity}

PrestoBlue assay was used to evaluate the effect of different concentrations of PCL-DPP-PCL NPs on cell viability in HuH-7 and MSCs. Both cells were seeded on a $96-$ well plate (10000 cells per well) and cultured at $37{ }^{\circ} \mathrm{C}$ with $5 \% \mathrm{CO}_{2}$. After $12 \mathrm{~h}$ for attachment, PCL-DPP-PCL NPs at four indicated concentrations were added to the medium. After incubation for 24 and $72 \mathrm{~h}$, respectively, the culture medium was removed and cells were washed three times by $1 \times$ PBS to remove excess NPs. Then, PrestoBlue reagent diluted by DMEM were added to each wells and incubated at $37^{\circ} \mathrm{C}$ with $5 \% \mathrm{CO}_{2}$. At the same time, PrestoBlue reagents diluted by DMEM were also added to blank wells without cells as control. After $1 \mathrm{~h}$ incubation, supernatant in each wells were transferred into another new 96-well plate in order to reduce the absorbance background of NPs. The absorbance at $571 \mathrm{~nm}$ (reference wavelength is $600 \mathrm{~nm}$ ) was detected by Plate Reader (Tecan Infinite M200 series Pro, Tecan Asia, Singapore). Percentage reduction of PrestoBlue reagent 
of each sample was calculated according to the following formula provided in manufacturer's protocol.

\subsection{Results and discussion}

\subsubsection{Preparation and characterization of NPs}

Figure. 2-1 shows the molecular structure of PCL-DPP-PCL and a schematic presentation of the NPs formed through a nanoprecipitation process. ${ }^{23-26}$ In this process, an aliquot of PCL-DPP-PCL dissolved in tetrahydrofuran (THF) (3 $\mathrm{mg} / \mathrm{mL}$ ) was added to a vigorously stirred solution of Pluronic ${ }^{\circledR} 127$ in water. Self-assembly was driven by the hydrophobic interaction between PCL-DPP-PCL and the polypropylene oxide (PPO) block of Pluronic ${ }^{\circledR} 127$ which collapsed to form the core of the NPs. The shell of the NPs consists of PEG chains originated from Pluronic ${ }^{(B)} 127$ that extrude to the aqueous media to provide the colloidal stability.

A representative scanning electron microscopy (SEM) image (Figure. 2-2a) shows that the resulting particles appear spherical and uniform in size and shape. A statistical analysis of the particle size gives an average diameter of 51 $\pm 4 \mathrm{~nm}$. Assuming a density of $1.14 \mathrm{~g} \cdot \mathrm{cm}^{-3}$ for PCL at room temperature and neglecting the contribution of Pluronic ${ }^{\circledR} 127$ to the diameter of each NP, the estimated average mass of each NP $(d=51 \mathrm{~nm})$ was $4.77 \times 10^{4} \mathrm{KDa}$, and that in average 1,240 polymer chains of PCL-DPP-PCL $\left(M_{\mathrm{n}}=38,300 \mathrm{Da}\right)$ existed in the core of each NP. While we observed that the size of the NPs could be tuned 
by changing the concentration of the polymer in THF, the following results and discussion will only focus on NPs with an average diameter of $51 \mathrm{~nm}$, which has been recently demonstrated to be within the optimal size range in monodisperse drug-silica nanoconjugates for deep tissue penetration and high retention in tumors. ${ }^{27}$ Figure. $2-2 b$ shows that the internal structure of the PCL-DPP-PCL NPs appeared amorphous as measured with transmission clectron microscopy (TEM). In addition, the size and shape of the NPs measured with TEM were consistent with those measured with SEM. Figure. $2-2 \mathrm{c}$ shows the result of dynamic laser light scattering (DLS) measurement, which gives an average diameter of $110 \mathrm{~nm}$ for the NPs dispersed in water. The size difference observed using electron microscopy and DLS, which has also been observed for other soft-matter particles, reflects the fact that the polymer NPs existed as a colloidal and swollen state in aqueous media, while they collapse and shrink to smaller sizes after being dried in air and imaged under the high-vacuum condition of electron microscopy.

Both the UV-vis absorbance (Figurc. 2-2e) and emission spectra (Figure. 2-2d) of the PCL-DPP-PCL NPs appear similar to those of a small-molecule DPP [2,5-bis(6-hydroxyhexyl)-3,6-di(thiophen-2yl)pyrrolo[3,4-c]pyrrole $-1,4(2 H, 5 H)$-dione $]$ (DPPHT) in dilute solution of THF. The NP dispersion in water shows characteristic absorbance peaks of DPP at 505 and $550 \mathrm{~nm}$, respectively, despite some light-scattering effect (Figure. 2-2e). The emission spectrum (Figure. 2-2d) of the PCL-DPP-PCL NP dispersion shows a major emission peak located at $560 \mathrm{~nm}$, accompanied with a 
shoulder at $600 \mathrm{~nm}$. There is a slight red shift $(3 \mathrm{~nm})$ of the maximum emission peak in the NP dispersion compared to that of DPPHT in THF. The fluorescence quantum yield of the PCL-DPP-PCL NPs in water is as high as

a

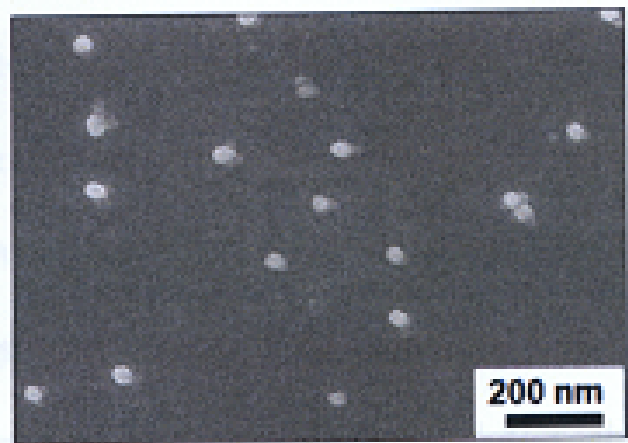

C

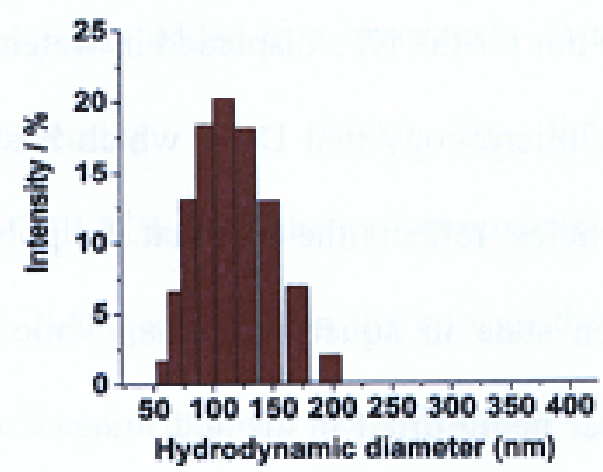

e

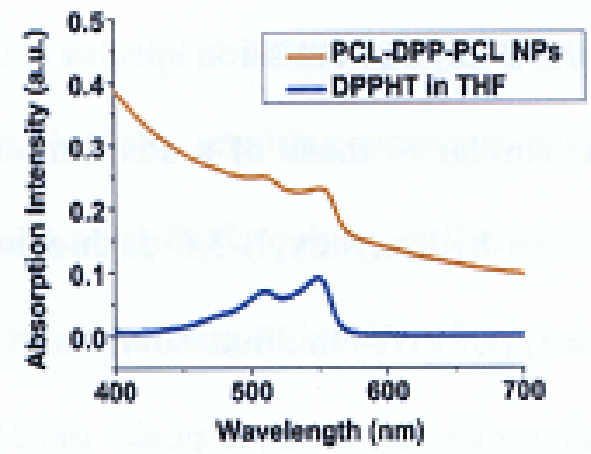

b

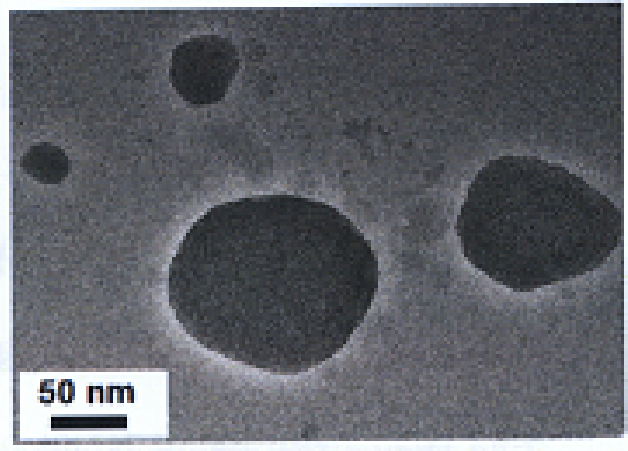

d

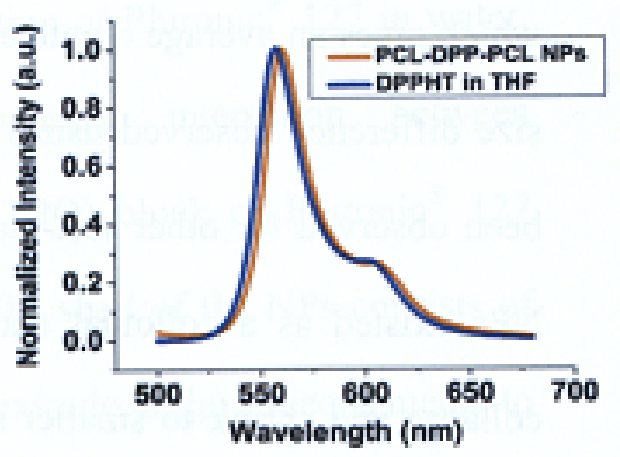

f

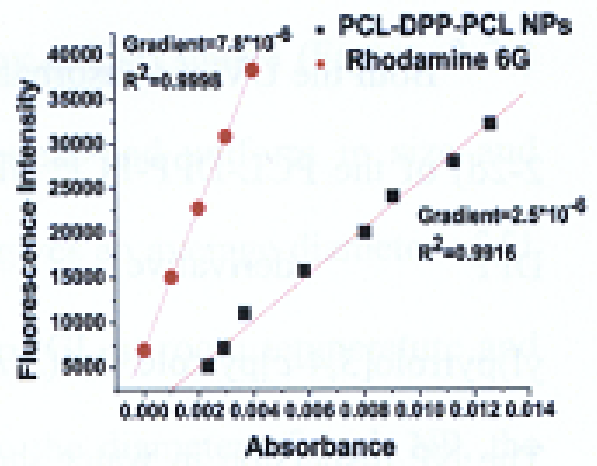

Figure 2-2. (a-f) The morphology characterizations and optical properties of PCL-DPP-PCL nanoparticles: (a) The SEM image showing the average particle size of $51 \pm 4 \mathrm{~nm}$. (b) TEM image of PCL-DPP-PCL nanoparticles from a water suspension. 
(c) The dynamic light scattering test shows that the hydrated NPs have an average diameter of $110 \mathrm{~nm}$ in water suspension. (d) The fluorescence emission spectra of PCL-DPP-PCL nanoparticles (orange) and DPPHT in THF (blue) under $488 \mathrm{~nm}$ excitation. (c) Absorbance spectra of PCL-DPP-PCL NPs and DPPHT in THF and (f) linear plots for standard samples for calculation of quantum yield and PCL-DPP-PCL NPs.

0.29 , based on Rhodamine $6 \mathrm{G}$ (quantum yield $=0.95$, in ethanol) ${ }^{28}$ as the standard (Figure. 2-2f). These results imply that the intermolecular $\pi-\pi$ aggregation among DPP units is minimal in the core of the PCL-DPP-PCL NPs. 


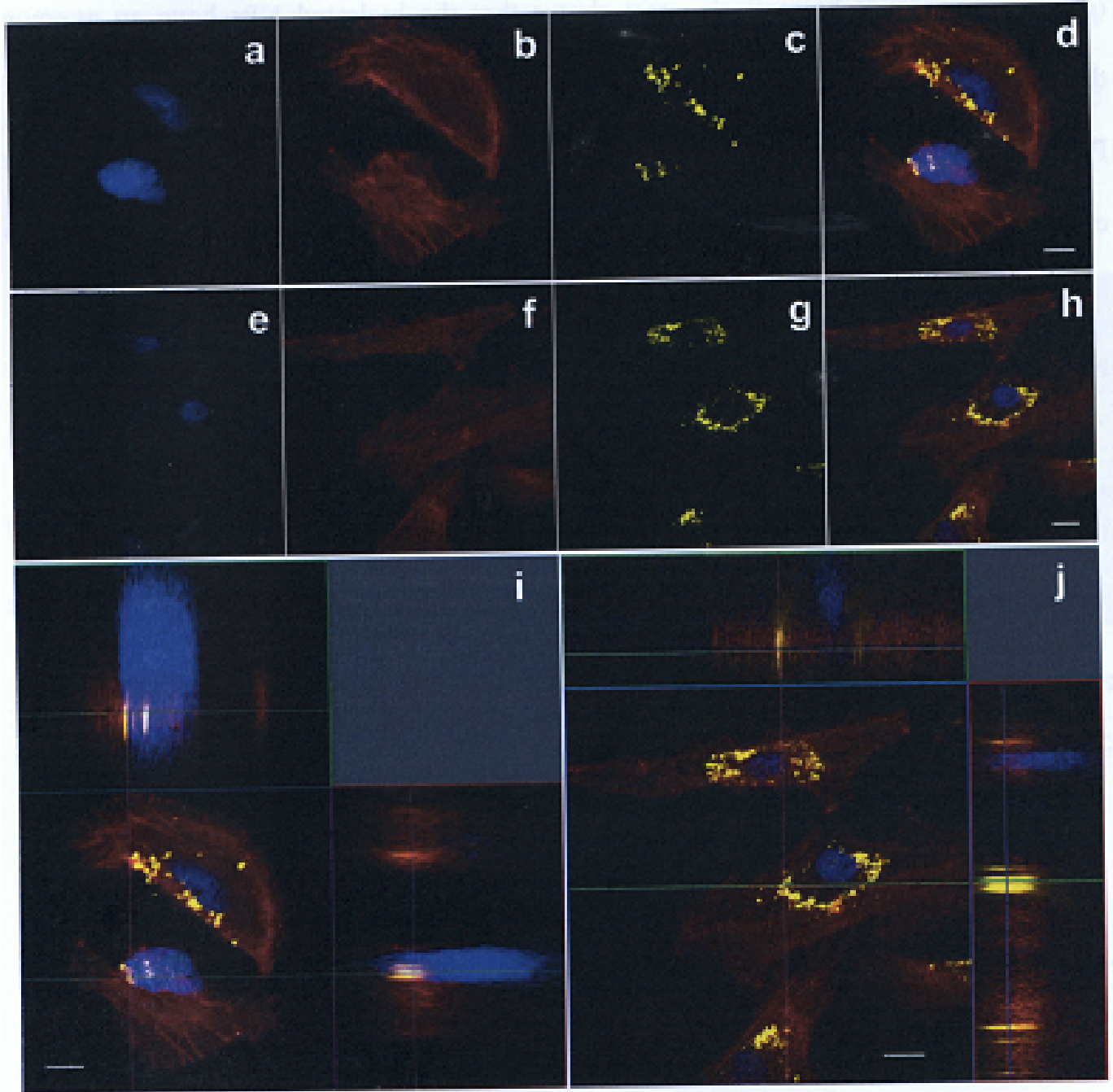

Figure 2-3. Cellular uptake of PCL-DPP-PCL nanoparticles (NPs) in HuH-7 tumor cells (a-d and i) and MSCs (e-h and j) imaged by confocal laser scanning microscopy. The fluorescence of DAPI, Alexa Fluor ${ }^{\circledR} 633$ phalloidin, and NPs are pseudo-labeled with blue (a, e), red (b, f), and yellow (c, g), respectively. Merged images of HuH-7 cells and MSCs from three channels are shown in (d) and (h), respectively. (i) and (j) are ortho-view images of z-stack, showing nanoparticles inside the cells. Scale bars: 10 $\mu \mathrm{m}(\mathrm{HuH}-7)$ and $20 \mu \mathrm{m}$ (MSC). 


\subsubsection{In vitro cellular uptake of NPs}

To explore the potential of these NPs in bioimaging application, we first studied the cellular uptake in vitro. Two types of cell lines were chosen to this end. HuH-7 is a hepatocyte derived carcinoma cell line that has been used as a popular model for cancer research and some transfection study on hepatological infectious diseases. ${ }^{29-31}$ MSCs are multipotent stromal cells that are able to differentiate into a variety of important cell lineages, and have become a promising cell source for regenerative medicine and tissue enginecring. ${ }^{32}$ Therefore we believe these two types of cells are both representative and biomedically relevant to characterize the prepared PCL-DPP-PCL NPs for cell imaging. Figure. 2-3 shows the fluorescence images of these two types of cells observed from three channels corresponding to different fluorophores: blue (DAPI), yellow (PCL-DPP-PCL NPs), and red (Alexa Fluor ${ }^{(\mathbb{R}} 633$ phalloidin). The merged images from all three channels and the ortho-view of Z-stack confirm that the NPs could be internalized by both HuH-7 tumor cells (Figure. 2-3 a-d, and i) and MSCs (Figure. 2-3 e-h, and j). Interestingly, the images in Figure. 2-3 (a-d, and i) suggest that the HuH-7 tumor cell nearly finished the mitotic (M) phase or cytokinesis when the mother cell split into two containing roughly equal shares of cellular components. As shown in Figure. 2-3, the internalized NPs are mainly located in the perinuclear cytoplasm, while not exhibiting obvious affinity to any specific intracellular

organelle. For further applications targeting at specific subcellular 
compartments, it may be possible that the polvmeric shell of these NPs could be functionalized with various ligands, such as nuclear localization signals (NLS) to facilitate NP passage through nuclear pore complex (NPC) for gene delivery $^{33}$, or mitochondria-targeting probes to enhance specific drug delivery to mitochondria and improve the therapeutic efficacy. ${ }^{34}$

\subsubsection{Photostability of NPs in cellular imaging}

Photobleaching is always a major concern in bioimaging applications of organic fluorophores. To characterize the photostability of PCL-DPP-PCL NPs, we compared their performance with two popular commercial fluorophores, DAPI and Alexa Fluor ${ }^{\mathbb{B}} 633$ phalloidin, by a simultaneous photobleaching test for multiply-stained biological cells. After being stained with a cocktail of three dyes, MSCs were continuously exposed to 405, 561, and $633 \mathrm{~nm}$ laser irradiation and the fluorescence emission of each fluorophore was recorded at time points in 36.8 -second intervals for a total duration of about $12 \mathrm{~min}$. A band-pass filter combination was applied with bandwidths tuned to record the emission in three channels: blue (421-481 nm), yellow (569-621 nm), and red $(650-758 \mathrm{~nm})$. Figure. 2-4a shows the merged images of the same 
a
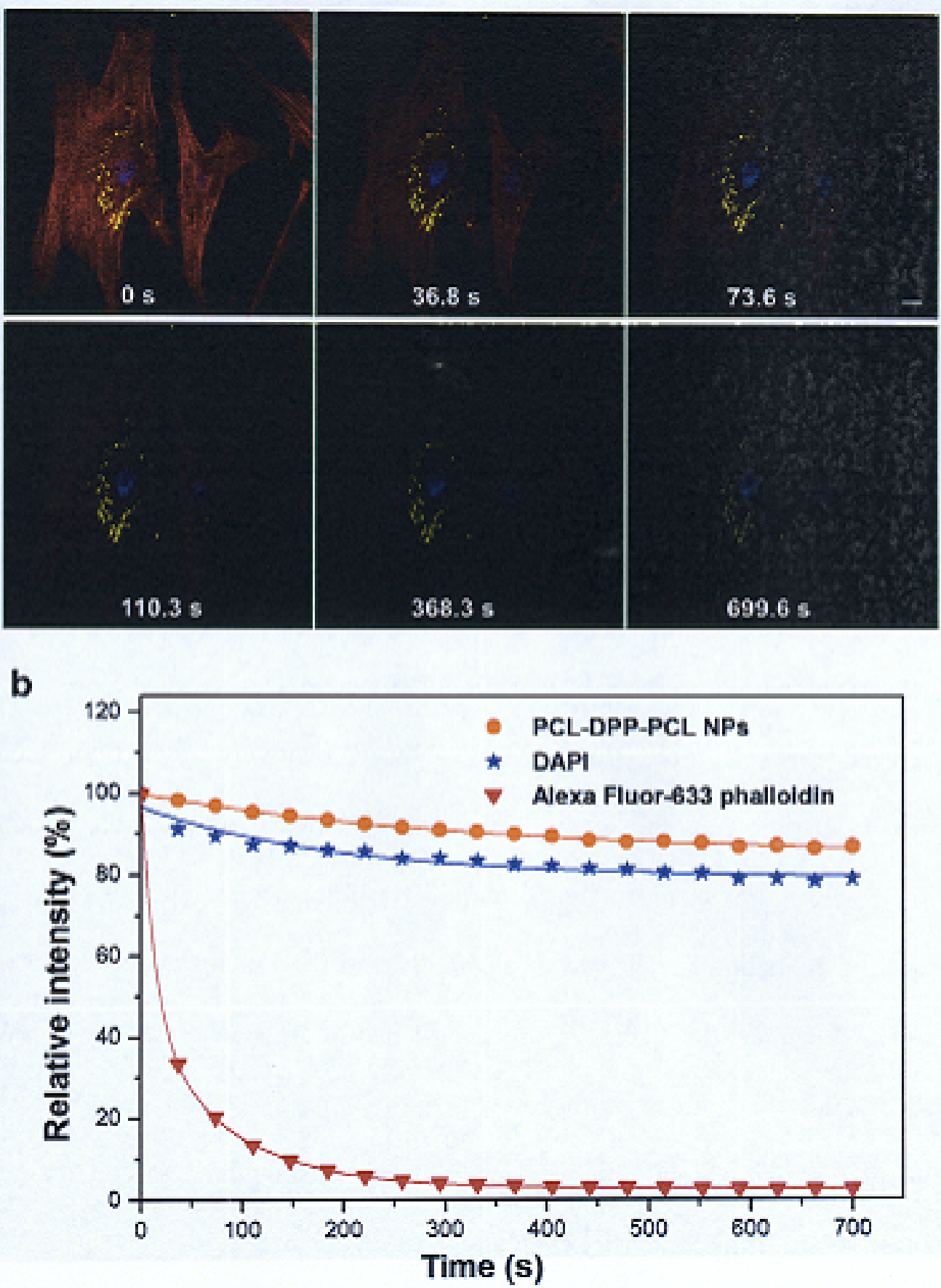

Figure 2-4. Photostability of fluorescent PCL-DPP-PCL nanoparticles (NPs) compared to Alexa Fluor ${ }^{\circledR} 633$ phalloidin and DAPI in MSC. The fluorescence of DAPI, NPs, and Alexa Fluor ${ }^{\circledR} 633$ phalloidin are pseudo-labeled with blue, yellow and 
red, respectively. (a) Chronological decay of fluorescence intensity of a multiply-stained MSC at different time points imaged by confocal laser scanning microscopy. The whole image field was photobleached. Pixel dwell time: $0.50 \mu$ s; frame size: $1024 \times 1024$; pixel size: $0.28 \mu \mathrm{m}$. Scale bar: $20 \mu \mathrm{m}$. (b) Relative intensity (instantaneous intensity / initial intensity) change of three fluorophores in the photobleached region. Curves are fitted using mono-exponential (PCL-DPP-PCL NPs and DAPI) or bi-exponential functions (Alexa Fluor ${ }^{\circledR} 633$ phalloidin). The nominal powers of 405, 561, and $633 \mathrm{~nm}$ lasers are $20 \mathrm{~mW}, 20 \mathrm{~mW}$, and $5 \mathrm{~mW}$, respectively.

multiply-stained MSC and the chronological decay of fluorescence intensity. A more detailed time-lapse of photobleaching, including those recorded through three individual fluorescence channels, is provided in Figure. 2-5. It can be observed that all three fluorophores showed relatively high fluorescence intensity at the beginning of the test, while the subsequent photobleaching of three fluorophores were not synchronous. The fluorescence intensity of Alexa Fluor $^{\circledR} 633$ phalloidin decayed most rapidly and the F-actin cytoskeleton was barely detectable after $110 \mathrm{~s}$ (Figure. 2-5b). The fluorescence intensity of DAPI and NPs decreased much more slowly (Figure. 2-5c, d). Notably, the NPs were able to maintain strong fluorescence intensity during the test and maintain almost $90 \%$ of initial intensity even after 11 min (Figure. 2-5d). 


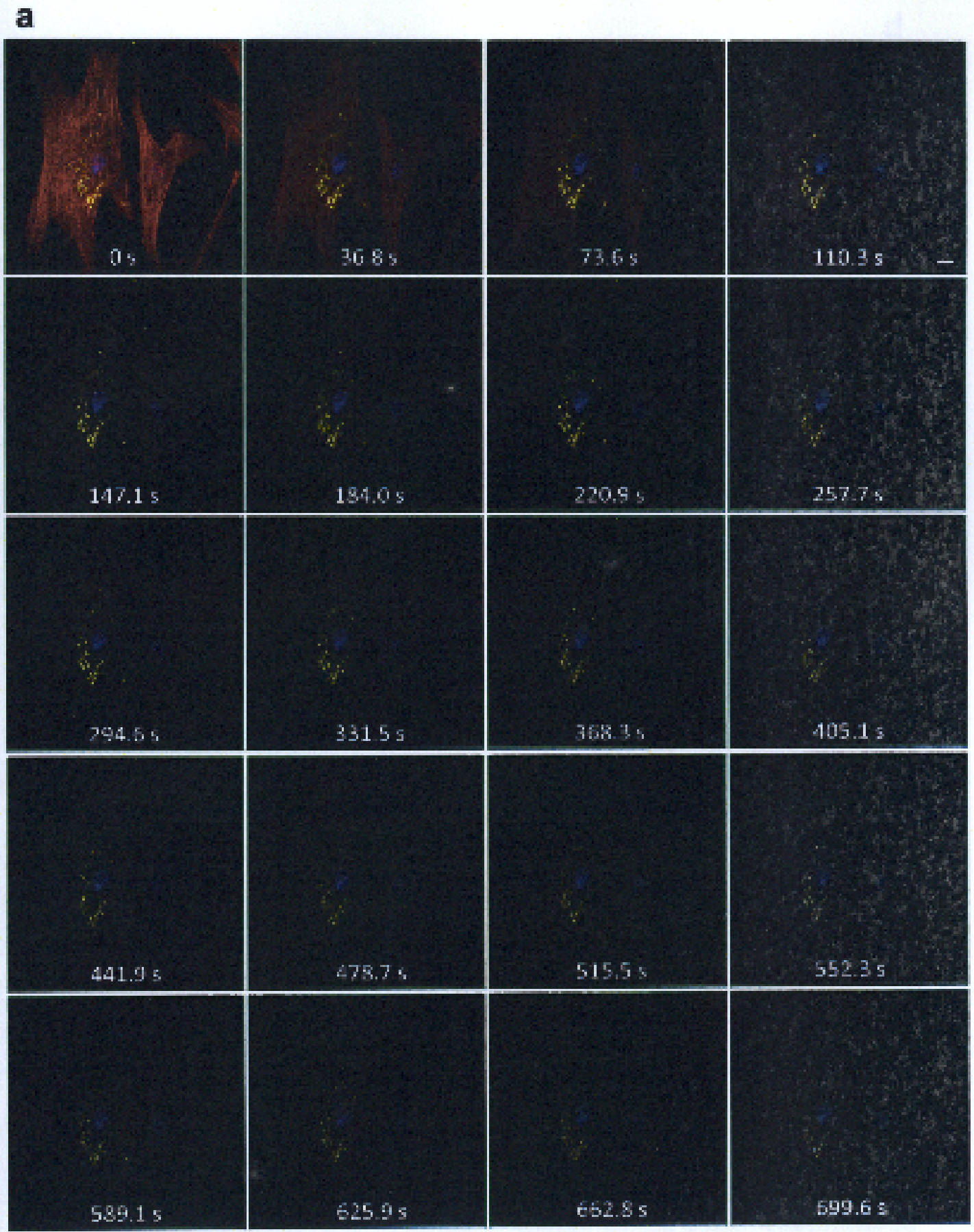




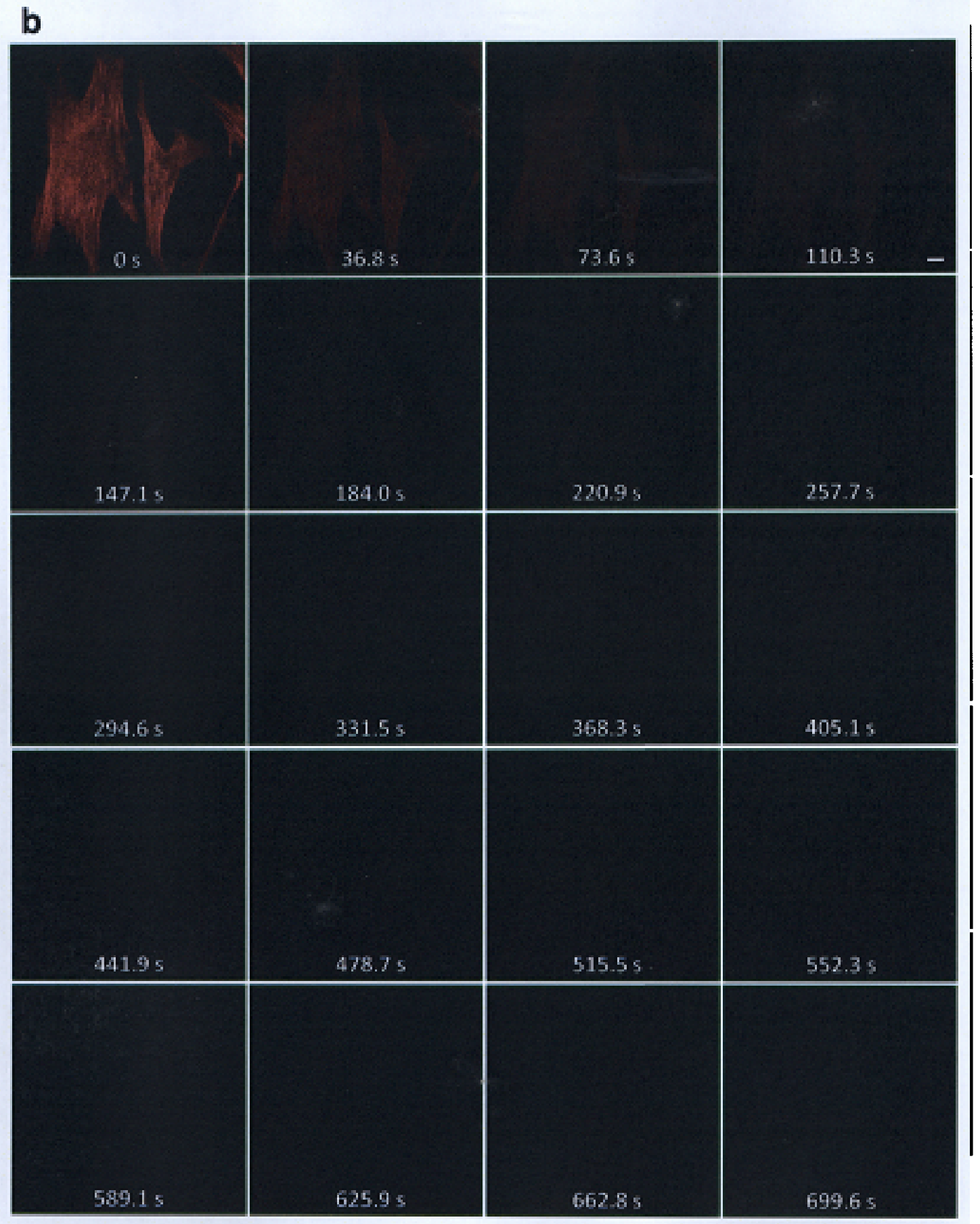




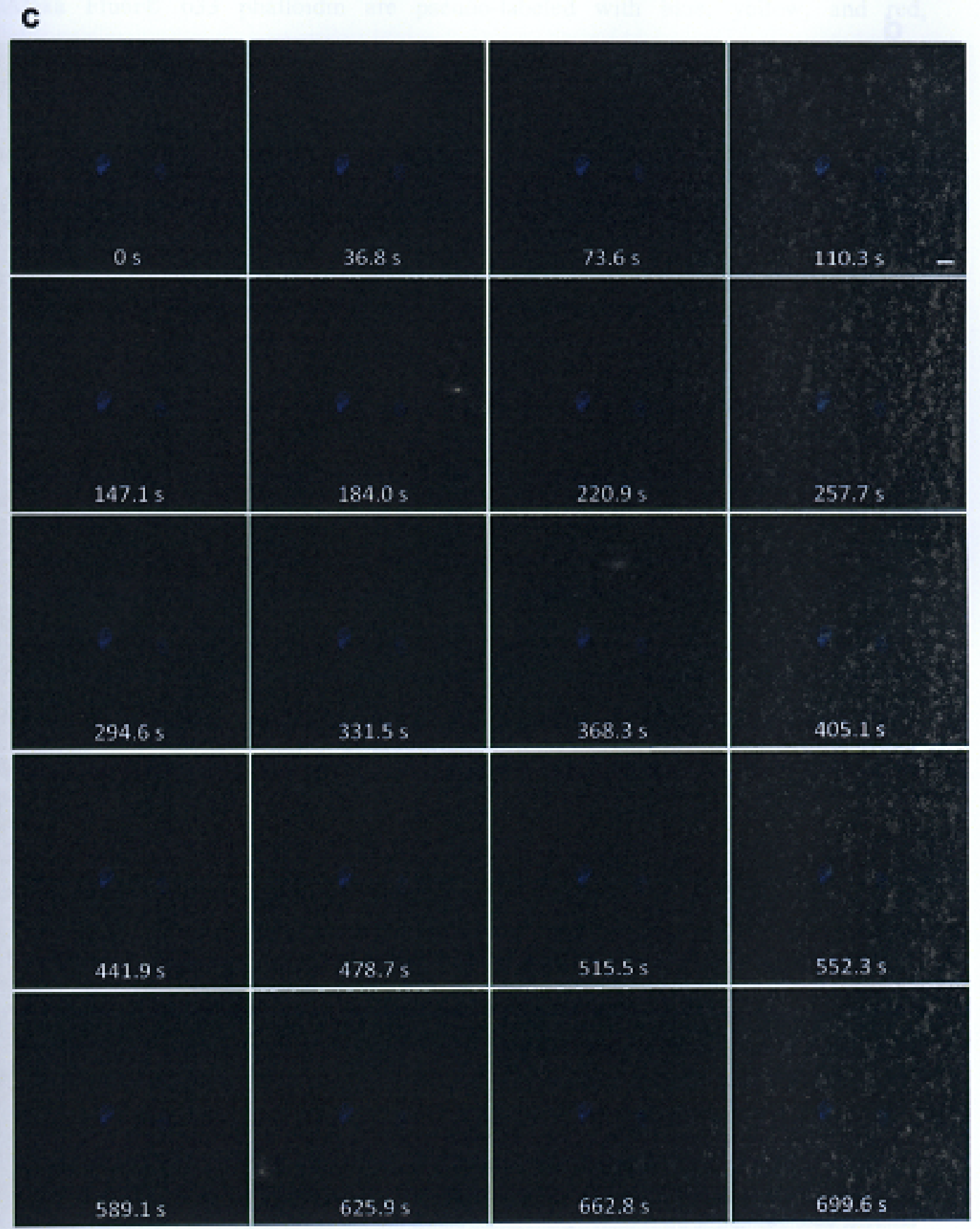




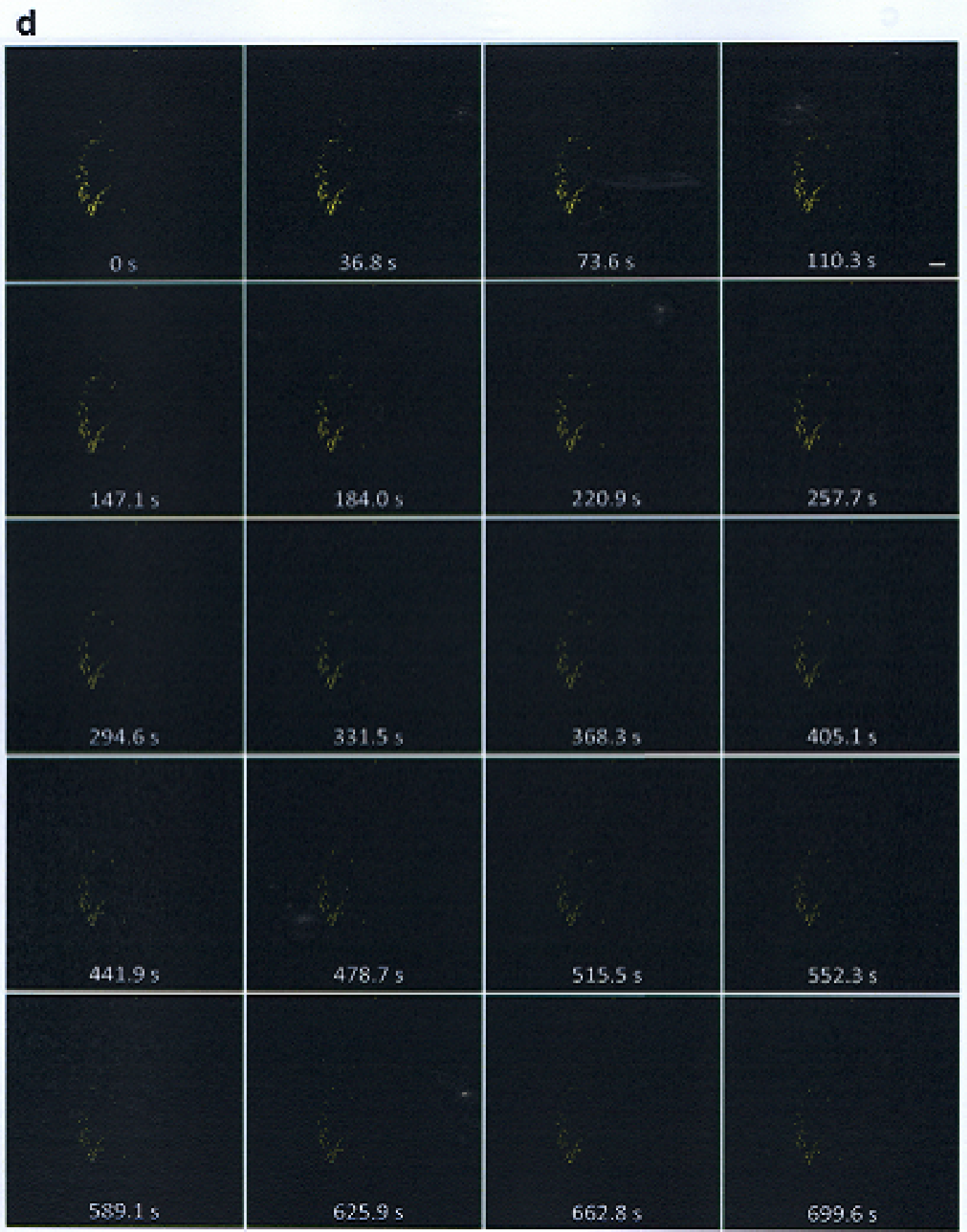

Figure 2-5. More detailed chronological decay (at $53 \mathrm{~s}$ intervals) of fluorescence intensity of merged images (a) and those from three individual channels: (b) Alexa Fluor ${ }^{\circledR} 633$ phalloidin, (c) DAPI and (d) NPs. The fluorescences of DAPI, NPs, and 
Alexa Fluor ${ }^{\circledR} 633$ phalloidin are pseudo-labeled with blue, yellow, and red, respectively. Scale bar: $20 \mu \mathrm{m}$.

A quantitative analysis of the relative intensity change (Figure 2-5) by curve fitting using exponential functions provided more insight of the decay kinetics of three different fluorophores. The PCL-DPP-PCL NPs exhibited mono-exponential decay expressed by $\left[y=A_{1} * \exp \left(-t / \tau_{1}\right)+y_{0}\right]$, where $\left(A_{1}+y_{0}\right)$ represents the initial relative intensity, $\mathrm{y}_{0}$ is the relative residual fluorescence intensity, and $\tau_{1}$ is the decay rate constant called mean lifetime. The half-life was calculated as $\ln (2)^{*} \tau_{1}$ (Table 2-1). DAPI also showed mono-exponential decay (Table 2-2) as reported in some previous studies. ${ }^{35,36}$ Although there was no prior study reporting the decay kinetics of Alexa Fluor ${ }^{\circledR} 633$ phalloidin, the fitting results showed that it could be described by a bi-exponential model $\left[y=A_{1} * \exp \left(-t / \tau_{1}\right)+A_{2} * \exp \left(-t / \tau_{2}\right)+y_{0}\right]($ Table 2-3) in MSC. In this bi-exponential model, $\left(A_{1}+A_{2}+y_{0}\right)$ represent the initial relative intensity; the half-life was computed based on the longer mean lifetime (Table 2-3). A comparison of the half-life values of three fluorophores in MSC (Tables 2-1 to 2-3) clearly showed that NPs (230 s) exhibited significantly higher photostability compared to DAPI (132 s) and Alexa Fluor ${ }^{\circledR} 633$ phalloidin (58.6 s).

Table 2-1. Parameters for fitting relative intensity change of PCL-DPP-PCL NPs in MSC (Figure 3b) using $y=A_{1} * \exp (-t / \tau 1)+y_{0}$

\begin{tabular}{ccc}
\hline & Value & Standard Error \\
\hline$y_{0}$ & 84.99 & 0.32 \\
\hline
\end{tabular}




\begin{tabular}{ccc}
\hline $\mathrm{A}_{1}$ & 14.97 & 0.28 \\
Mean lifetime $\tau_{1}(\mathrm{~s})$ & 331.82 & 17.28 \\
Adj. R-square & 0.9967 & \\
Half-life & 230.00 & \\
$\left(\mathrm{t}_{1 / 2}=\ln 2 * \tau_{1}, \mathrm{~s}\right)$ & & \\
\hline
\end{tabular}

Table 2-2. Parameters for fitting relative intensity change of DAPI in MSC (Figure $3 b$ ) using $\mathrm{y}=\mathrm{A}_{1} * \exp (-\mathrm{t} / \tau 1)+\mathrm{y}_{0}$

\begin{tabular}{ccc}
\hline & Value & Standard Error \\
\hline $\mathrm{y}_{0}$ & 79.64 & 0.80 \\
$\mathrm{~A}_{1}$ & 17.17 & 1.09 \\
\hline Mean lifetime $\tau_{1}(\mathrm{~s})$ & 190.62 & 32.56 \\
\hline Adj. R-square & 0.9288 & \\
Half-life & 132.13 & \\
$\left(\mathrm{t}_{1 / 2}{ }^{*}=\ln 2 * \tau_{1}, \mathrm{~s}\right)$ & & \\
\hline
\end{tabular}

Table 2-3. Parameters for fitting relative intensity change of Alex Fluor ${ }^{\circledR} 633$ in MSC (Figure $3 b$ ) using $y=A_{1} * \exp (-t / \tau 1)+A_{2} * \exp (-t / \tau 2)+y_{0}$

\begin{tabular}{ccc}
\hline & Value & Standard Error \\
\hline $\mathrm{y}_{0}$ & 2.80 & 0.06 \\
$\mathrm{~A}_{1}$ & 56.34 & 1.27 \\
\hline Mean lifetime $\tau_{1}(\mathrm{~s})$ & 14.28 & 0.68 \\
$\mathrm{~A}_{2}$ & 40.86 & 1.23 \\
\hline
\end{tabular}




\begin{tabular}{ccc}
\hline Mean lifetime $\tau_{2}(\mathrm{~s})$ & 84.56 & 2.02 \\
Adj. R-square & 0.9999 \\
Half-life $\left(\mathrm{t}_{1 / 2}{ }^{*}=\ln 2^{*} \tau_{2}\right.$, & $58.61 \mathrm{~s}$ \\
$\mathrm{~s})$ & \\
\hline
\end{tabular}

As discussed previously, small-molecule DPP derivatives often show fluorescence quenching in solid states due to significant intermolecular $\pi-\pi$ aggregation. The reason why PCL-DPP-PCL NPs exhibit much greater photostability could be due to the covalent bonds between DPP and PCL chains, which efficiently suppressed the aggregation of DPP molecules. In order to verify this hypothesis, we prepared PCL/DPPHT nanoparticles (Figure. 2-6 a-c) by simple physical blending (i.e. without any covalent bond formation between these two components $)$ of DPPHT $\left(8.0 \times 10^{-4} \mathrm{mmol}\right)$ and PCL $\left(M_{\mathrm{n}}=22,000\right.$, PDI $=1.3$ ) with a weight ratio similar to that of PCL-DPP-PCL. These physically blended PCL/DPP NPs could also be internalized by MSCs and accumulated in perinuclear region (Figure. 2-7 a-d, and i). However, photoblcach test revealed that these physically blended NPs exhibited much weaker photostability (Figure. 2-8) as compared to the covalently bonded PCL-DPP-PCL NPs (Figure. 2-4). After $125 \mathrm{~s}$ of laser irradiation $(561 \mathrm{~nm}$ ), physically blended NPs could only retain about $13 \%$ of initial fluorescence intensity. 

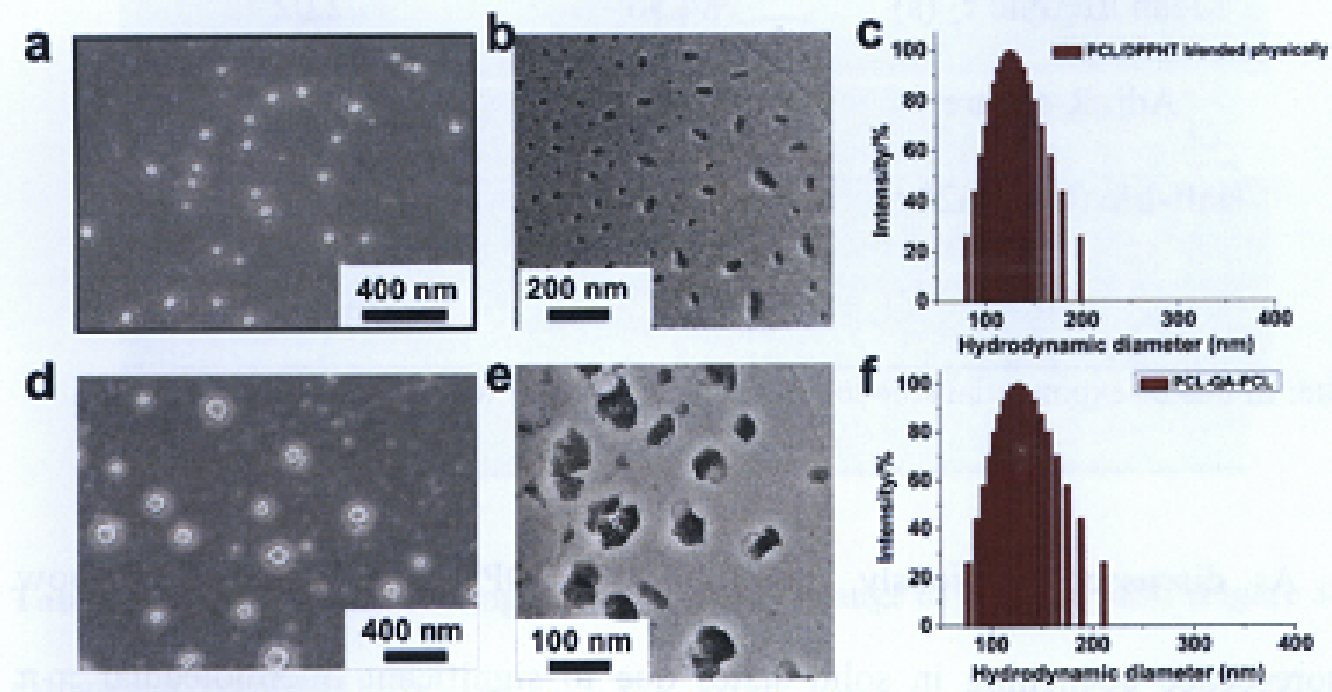

g

PCL-QA-PCL

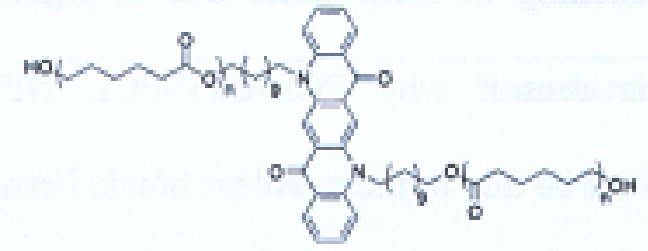

Figure 2-6. SEM (a, d), TEM (b, e) and DLS (c, f) characterization of physically blended DPPHT/PCL NPs (a-c) and PCL-QA-PCL NPs (d-f). (g) The chemical structure of PCL-QA-PCL. 


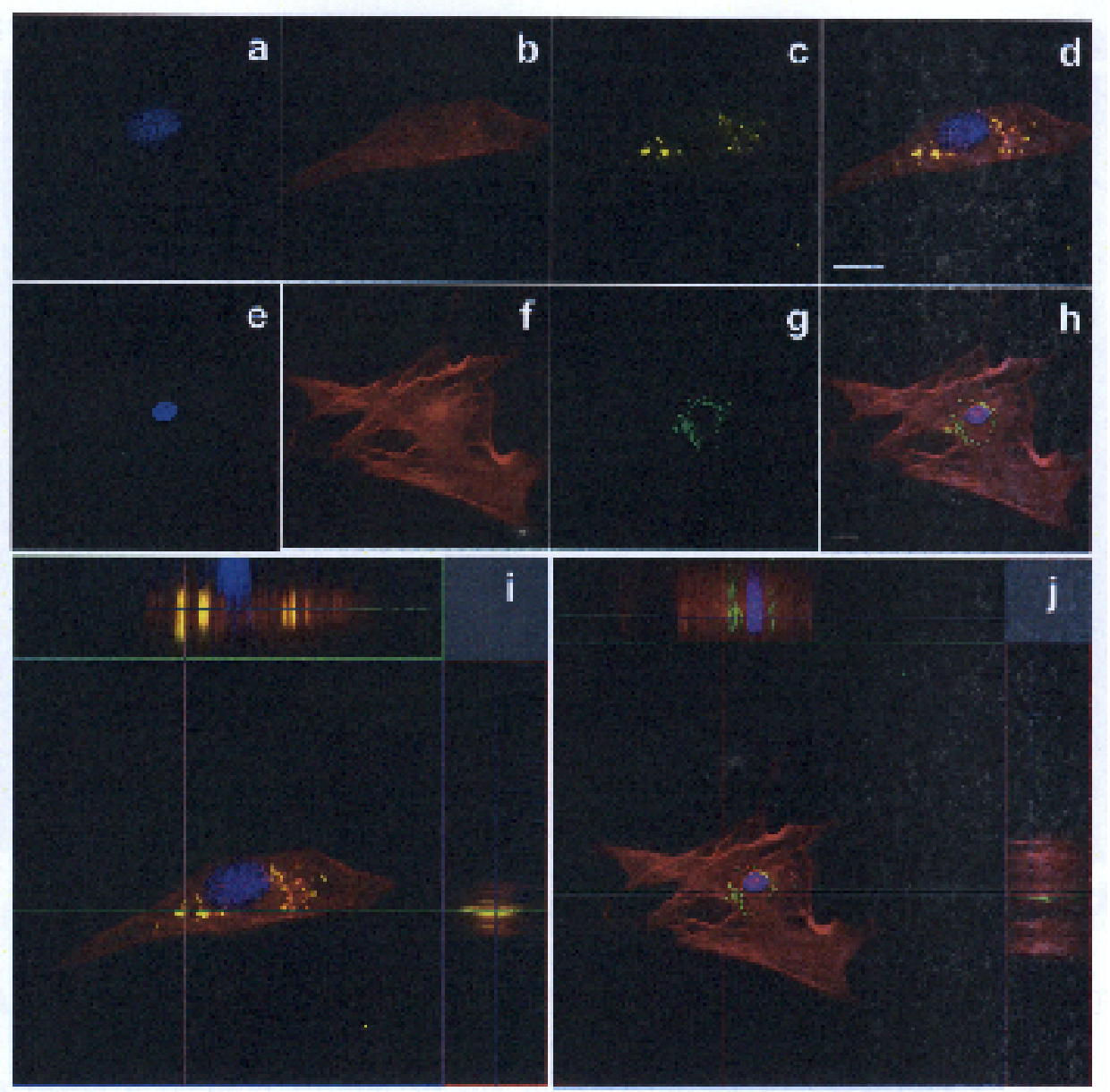

Figure 2-7. Cellular uptake of physically blended PCL/DPPHT NPs (a-d and i) and PCL-QA-PCL nanoparticles (e-h and j) in MSCs imaged by confocal laser scanning microscopy. The fluorescence of DAPI, Alexa Fluor ${ }^{\circledR} 633$ phalloidin, physically blended PCL/DPPHT NPs, and PCL-QA-PCL nanoparticles are pseudo-labeled with blue (a, e), red (b, f), yellow (c), and green (g), respectively. Merged images of MSCs from different channels are shown in (d) and (h), respectively. (i) and (j) are ortho-view images of z-stack, showing they could be uptaken by the cells. Scale bars: $20 \mu \mathrm{m}$. 

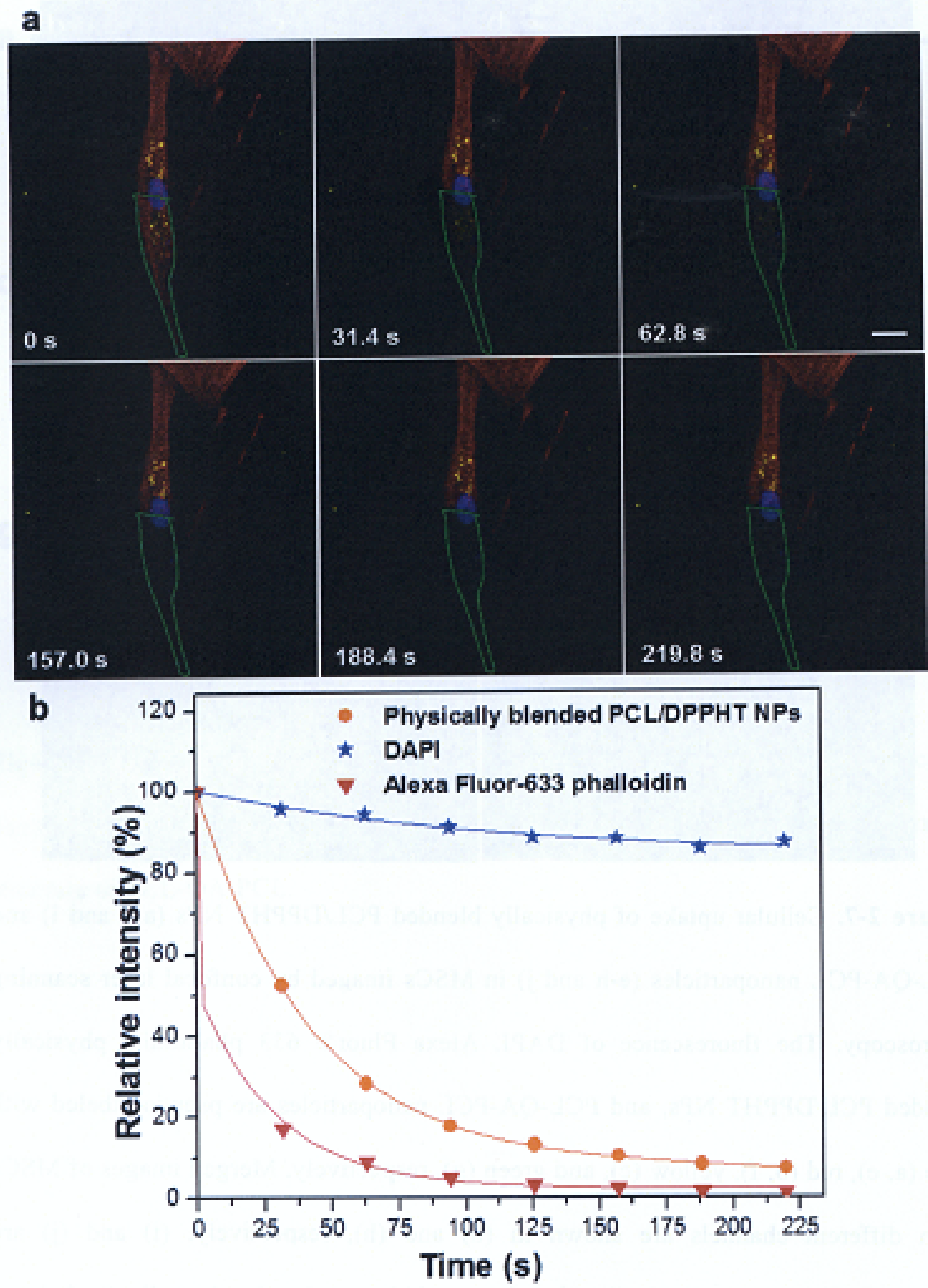

Figure 2-8. Photostability of physically blended PCL/DPPHT NPs compared to Alexa Fluor ${ }^{\circledR} 633$ phalloidin and DAPI in a MSC. The fluorescence of DAPI, physically blended PCL/DPPHT NPs, and Alexa Fluor ${ }^{\circledR} 633$ phalloidin are pseudo-labeled with blue, yellow and red, respectively. (a) Chronological decay of fluorescence intensity of 
a multiply-stained MSC at different time points imaged by confocal laser scanning microscopy. The green polygon indicates the region photobleached. Pixel dwell time: $1.27 \mu$ s; frame size: $512 \times 542$; pixel size: $0.37 \mu \mathrm{m}$. Scale bar: $20 \mu \mathrm{m}$. (b) Relative intensity (instantaneous intensity / initial intensity) change of three fluorophores in the photobleached region. Curves are fitted using mono-exponential (DAPI) or bi-exponential functions (physically blended PCL/DPPHT NPs and Alexa Fluor 833 phalloidin). The nominal powers of $405 \mathrm{~nm}, 561 \mathrm{~nm}$, and $633 \mathrm{~nm}$ lasers were $20 \mathrm{~mW}$, $20 \mathrm{~mW}$, and $5 \mathrm{~mW}$, respectively.

In addition to DPP-based fluorophores, this strategy to enhance the photostability could also be applied to other fluorophores with different excitation and emission wavelengths. As an example, we chose a quinacridone (QA) derivative (Figure. 2-6g) as an initiator and synthesized PCL-QA-PCL NPs using a method similar to the preparation of PCL-DPP-PCL NPs. The SEM image (Figure. 2-6d) showed that the resulting spherical particles had an average diameter of $117 \pm 21 \mathrm{~nm}$, which was consistent with the DLS (Figure. 2-6f) and TEM (Figure. 2-6e) results. Fluorescence microscopy (Figure. 2-7 e-h, and j) showed that they could be uptaken by MSCs and accumulated in the perinuclear region. The photobleach test suggested that the photostability of PCL-QA-PCL NPs (Figure 2-9) was relatively weaker than that of PCL-DPP-PCL NPs (Figure. 2-4), which could be due to different properties of QA and DPP. 


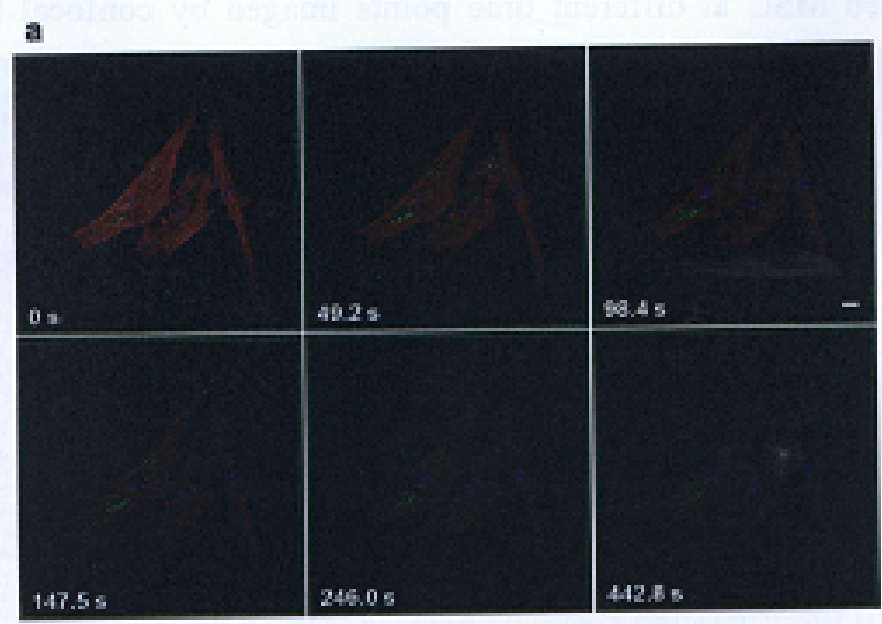

b

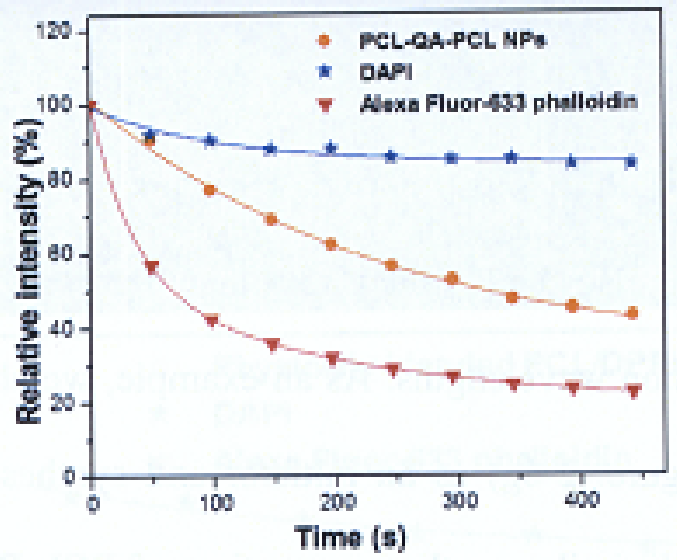

Figure 2-9. Photostability of PCL-QA-PCL NPs compared to Alexa Fluor ${ }^{\circledR} 633$ phalloidin and DAPI in a MSC. The fluorescence of DAPI, PCL-QA-PCL NPs, and Alexa Fluor ${ }^{\circledR} 633$ phalloidin are pseudo-labeled with blue, green and red, respectively. (a) Chronological decay of fluorescence intensity of a multiply-stained MSC at different time points imaged by confocal laser scanning microscopy. The whole image field was photobleached. Pixel dwell time: $0.64 \mu$ s; frame size: $1024 \times 1024$; pixel size: $0.42 \mu \mathrm{m}$. Scale bar: $20 \mu \mathrm{m}$. (b) Relative intensity (instantaneous intensity / initial intensity) change of three fluorophores in the photobleached region. Curves are fitted using mono-exponential (DAPI) or bi-exponential functions (PCL-QA-PCL NPs and Alexa Fluor ${ }^{\circledR} 633$ phalloidin). The nominal powers of $405 \mathrm{~nm}, 488 \mathrm{~nm}$, and $633 \mathrm{~nm}$ lasers were $25 \mathrm{~mW}, 25 \mathrm{~mW}$, and $5 \mathrm{~mW}$, respectively. 

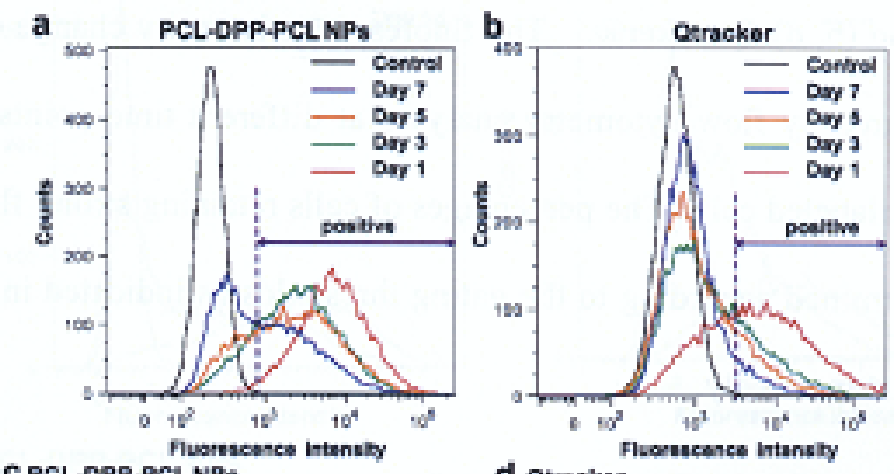

C PCL-OPP.PCL NPa

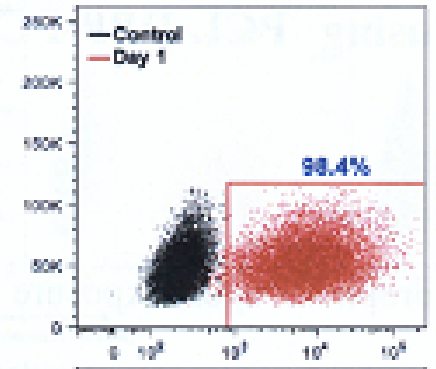

d atracker
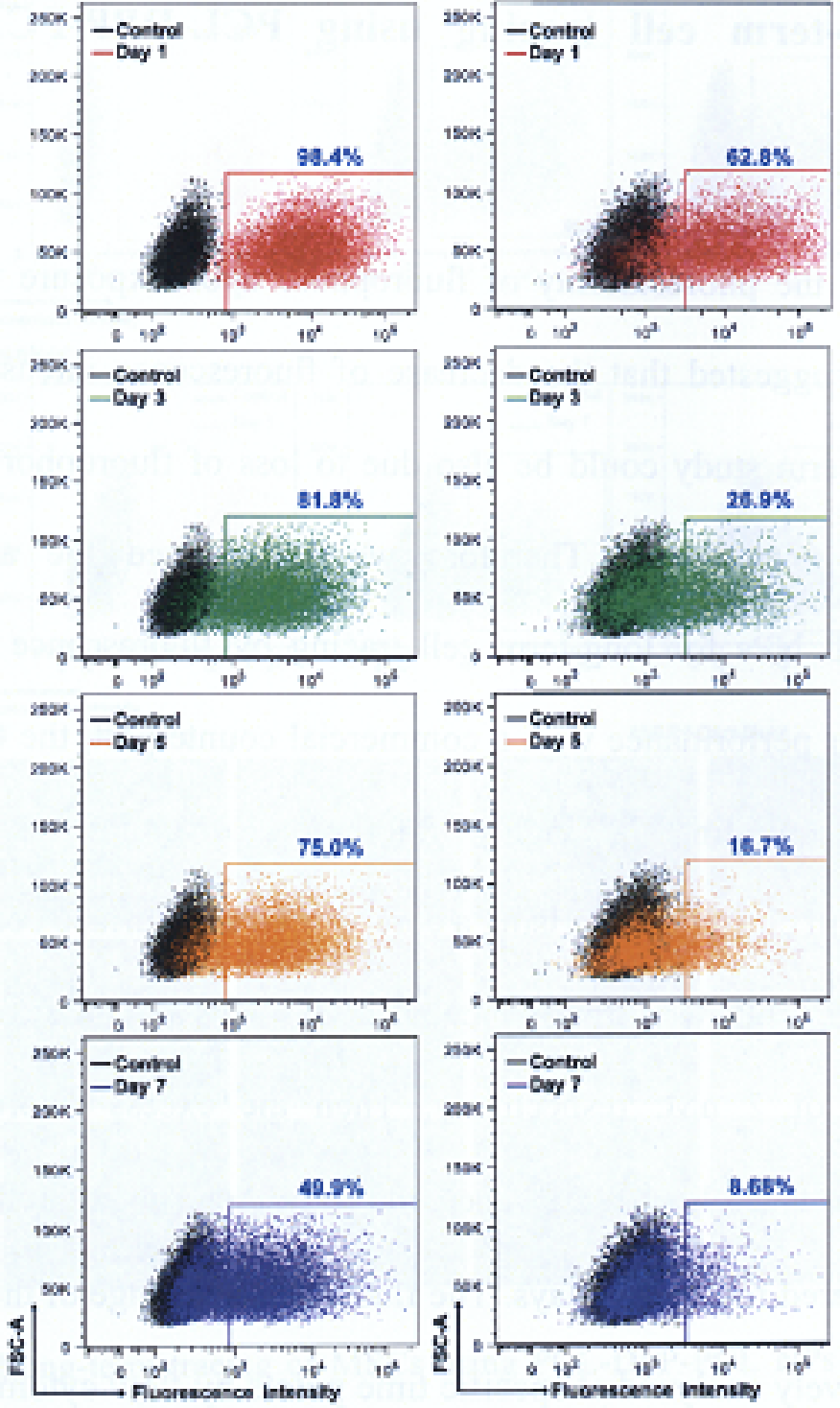
Figure 2-10. Long-term tracing of HuH-7 tumor cells using (a, c) PCL-DPP-PCL nanoparticles and (b, d) Qtracker ${ }^{\circ}$. The fluorescence intensity change of the labeled cells was measured by flow cytometry analyses at different time points. The control represents the unlabeled cells. The percentages of cells retaining strong fluorescence in (c, d) were determined according to the gating thresholds as indicated in (a, b) for NP and Qtracker®, respectively.

\subsubsection{Long-term cell tracing using PCL-DPP-PCL NPs vs.}

\section{Qtracker $^{\circledR}$}

In addition to the photostability of fluorophore upon exposure to light, prior studies have suggested that the decrease of fluorescence intensity of stained cells in long-term study could be also due to loss of fluorophores during cell division and exocytosis ${ }^{37}$. Therefore we investigated the application of PCL-DPP-PCL NPs for long-term cell tracing by fluorescence imaging, and compared their performance with a commercial counterpart, the Qtracker ${ }^{\circledR}$ cell labeling kit consisting of highly fluorescent quantum dot nanocrystals (nanoscale atom clusters comprising a core, a shell and surface coating).

HuH-7 tumor cells were firstly incubated with NPs and Qtracker ${ }^{\circledR}$ for $48 \mathrm{~h}$ at concentration of $2 \mathrm{nM}$, respectively. Then the excess fluorophores were removed and washed by $1 \times \mathrm{PBS}$. The cells were then subcultured into a 6 -well plate and cultured for up to 7 days. The fluorescence change of the labeled cells was quantitatively analyzed at specific time points by flow cytometry for about 10,000 cell events in each test (Figure. 2-10). The fluorescence of HuH-7 cells 
a

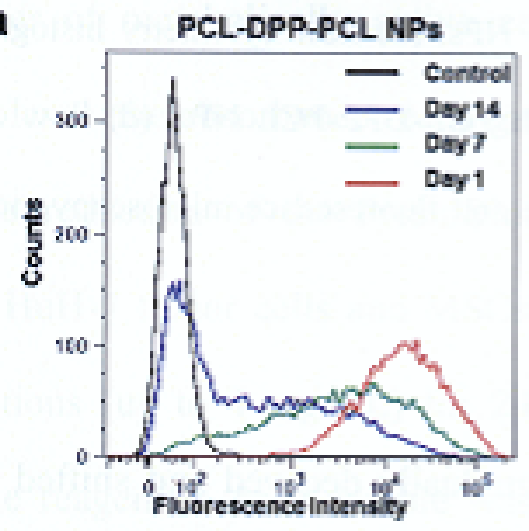

b

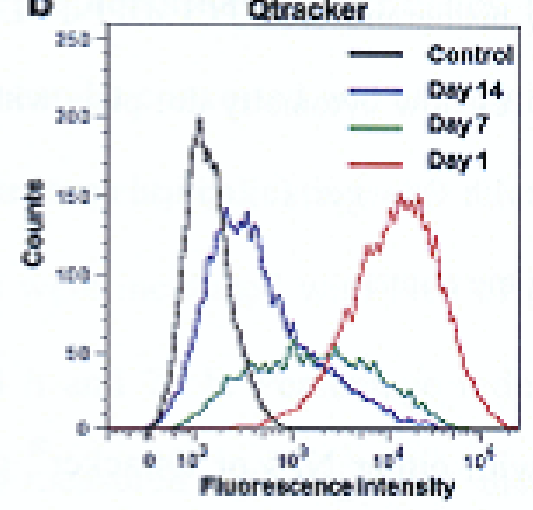

C PCL-DPP.PCL. NPs
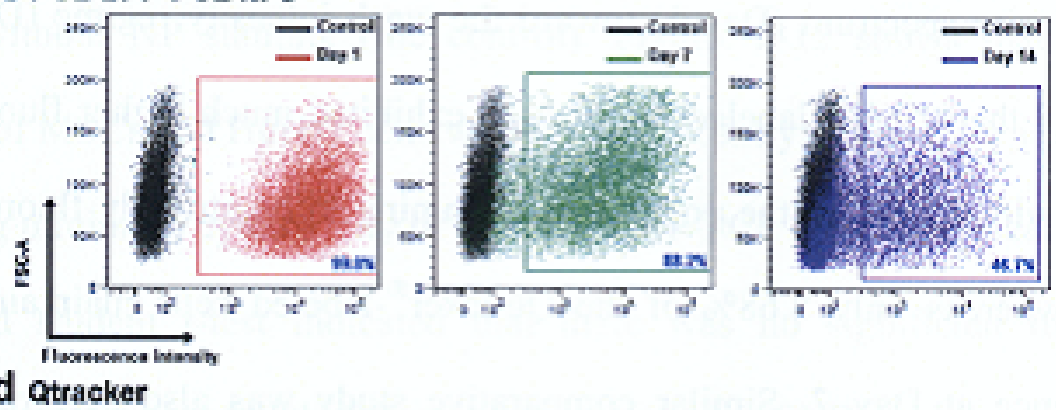

d Qtracker
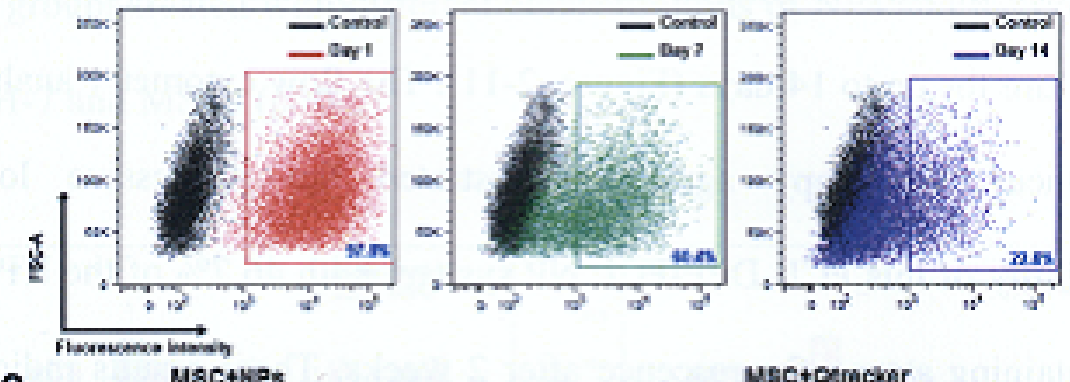

e

MSC+kPs

MsctQtracion
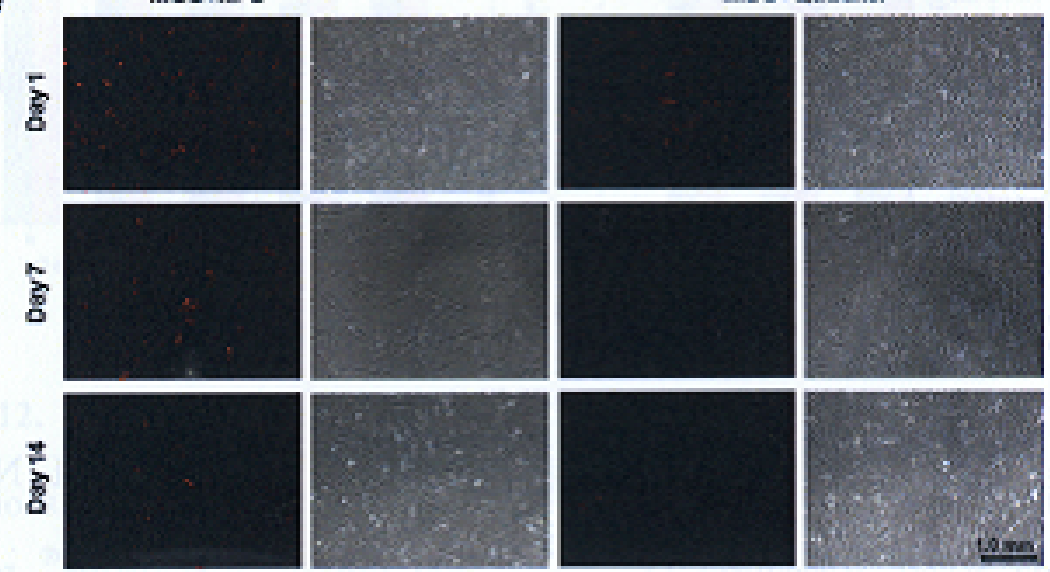

Figure 2-11. Long-term tracing of MSCs using PCL-DPP-PCL NPs and Qtracker®.

The fluorescence intensity change of the labeled cells is characterized by (a) flow 
cytometry histograms with PCL-DPP-PCL NPs, (b) flow cytometry histograms with Qtracker, (c) flow cytometry dot plots with PCL-DPP-PCL NPs, (d) flow cytometry dot plots with Qtracker, (e) optical contrast and fluorescence microscopy measured at different time points.

labeled with either NPs or Qtracker ${ }^{\circledR}$ gradually decayed and shifted from the high intensity spectrum (Day 1) toward the weak intensity regime (Day 7). It was noted that the NP-labeled tumor cells exhibited much higher fluorescence stability with $49.9 \%$ of the population remaining to be strongly fluorescent at Day 7; whereas only $8.68 \%$ of the Qtracker ${ }^{\mathbb{R}}$-labeled cells maintained their fluorescence at Day 7. Similar comparative study was also performed with MSC culture for up to 14 days (Figure. 2-11). The flow cytometry analysis and fluorescence microscopy again demonstrated the impressive long-term photostability of this PCL-DPP-PCL NP system, with $46.7 \%$ of the NP-stained MSCs retaining strong fluorescence after 2 weeks. These results indicate that the PCL-DPP-PCL NPs are highly advantageous as a stable fluorophore particularly for applications related to long-term cell tracing.

\subsubsection{Cytotoxicity of PCL-DPP-PCL NPs.}

Finally, we evaluated the cytotoxicity of the PCL-DPP-PCL NPs using PrestoBlue assay. Resazurin $\left(\lambda_{\text {max.abs }}=600 \mathrm{~nm}\right)$ in PrestoBlue ${ }^{\circledR}$ reagent, a nonfluorescent blue compound, can be reduced in live cells by metabolism to resorufin $\left(\lambda_{\text {max.abs }}=571 \mathrm{~nm}\right)$, which is red in color and highly fluorescent. Since 
the number of metabolically active cells proportionally correlates with the reduction level, the absorbance readings can be converted and expressed as the percentage reduction of the PrestoBlue reagent, indicating the relative cell viability. HuH-7 tumor cells and MSCs were incubated with NPs of different concentrations (up to $4 \mathrm{mg} / \mathrm{mL}$ ) for $24 \mathrm{~h}$ and $72 \mathrm{~h}$. Percentage reduction of PrestoBlue reagent in each sample was measured with regard to that in cell culture without NP staining (the control). Figure. 2-12 shows that the cell viability of MSCs and HuH-7 cells was not significantly affected for up to $72 \mathrm{~h}$, indicating minimum cytotoxicity of the PCL-DPP-PCL NPs. Statistical analysis by paired student $t$-test indicated that there was no significant difference between groups treated with different concentrations of NPs and the control for both HuH-7 and MSCs ( $>0.5)$.
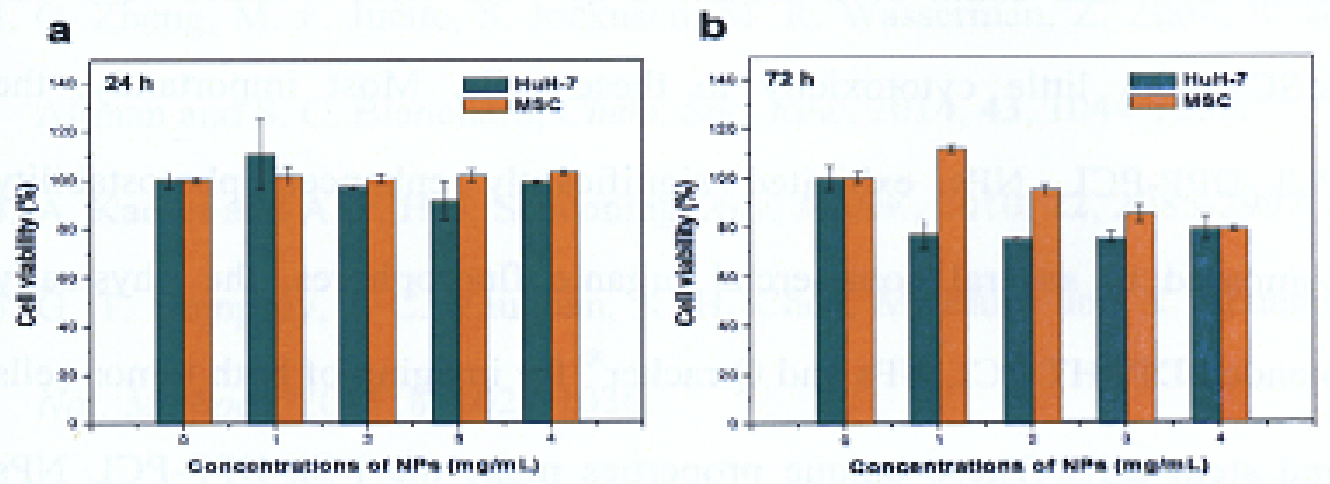

Figure 2-12. Cell viabilities of $\mathrm{HuH}-7$ and MSCs after incubating with different concentrations of PCL-DPP-PCL NPs for (a) $24 \mathrm{~h}$ and (b) $72 \mathrm{~h}$ tested by PrestoBlue assay. Cells without treatment by NPs were used as control. Values were expressed as means \pm SD $(\mathbf{n}=3)$. Paired student $\mathrm{t}$-test was used for statistical analysis between groups treated with different concentrations of NPs and control $(\mathrm{p}>0.5)$. 


\subsection{Conclusions}

In summary, we have presented a new type of highly fluorescent and bioresorbable polymeric NPs prepared through nanoprecipitation of a well-defined polymer, PCL-DPP-PCL, in the presence of Pluronic ${ }^{\mathbb{B}} 127$ as the stabilizer. A key feature of our molecular design lies in the covalent insertion of DPP as a highly fluorescent moiety into the middle of each PCL chain. Such covalent bonding at the interface of DPP and PCL as well as the steric effect endowed by long PCL chains effectively suppress the $\pi-\pi$ aggregation of DPP in the PCL matrix. As a consequence, PCL-DPP-PCL with $M_{\mathrm{n}}$ of 38,300 Da showed bright fluorescence in solid states. The NPs prepared through a nanoprecipitation process were highly fluorescent and could be internalized by both $\mathrm{HuH}-7$ tumor cells and MSCs with little cytotoxicity to these cells. Most importantly, the PCL-DPP-PCL NPs exhibited significantly enhanced photostability compared to several commercial organic fluorophores, the physically blended DPPHT/PCL NPs and Qtracker ${ }^{\circledR}$ for imaging of both tumor cells and stem cells. These unique properties make the PCL-DPP-PCL NPs promising imaging probes to track a broad range of biological systems. Compared to previously reported fluorescent organic or polymeric NPs, ${ }^{16,}$ ${ }^{17}$ the $1.3 \%$ weight fraction of fluorescent components in these NPs is significantly lower and the polymer matrix (i.e. PCL) has proved to be biocompatible and bioresorbable. Therefore the potential toxicity, if any, 
caused by the encapsulated fluorophores is minimized. Moreover, the fluorescence excitation and emission wavelengths of these synthetic NPs can be customized and finely tuned during synthesis for specific applications. Currently we are extending this strategy to polymers with strong fluorescence in near infrared range that is important for in-vivo imaging.

\subsection{References}

1. in Principles of Fluorescence Spectroscopy, ed. J. Lakowicz, Springer US, Editon edn., 2006.

2. P.-C. Cheng, in Handbook of Biological Confocal Microscopy, ed. J. B. Pawley, Springer US, New York, Editon edn., 2006, pp. 162-206.

3. Q. Zheng, M. F. Juette, S. Jockusch, M. R. Wasserman, Z. Zhou, R. B. Altman and S. C. Blanchard, Chem. Soc. Rev., 2014, 43, 1044-1056.

4. A. Kacser and A. P. H. J. Schenning, Adv. Mater, 2010, 22, 2985-2997.

5. G. T. Dempsey, J. C. Vaughan, K. H. Chen, M. Bates and X. Zhuang, Nat. Methods, 2011, 8, 1027-1036.

6. A. Wagh, S. Y. Qian and B. Law, Bioconjug. Chem., 2012, 23, 981-992.

7. A. Reisch, P. Didier, L. Richert, S. Oncul, Y. Arntz, Y. Mély and A. S. Klymchenko, Nat. Commun., 2014, 5, 4089.

8. R. B. Altman, D. S. Terry, Z. Zhou, Q. Zheng, P. Geggier, R. A. Kolster, Y. Zhao, J. A. Javitch, J. D. Warren and S. C. Blanchard, Nat. Methods, $2012,9,68-71$. 
9. M. Bruchez, M. Moronne, P. Gin, S. Weiss and A. P. Alivisatos, Science, 1998, 281, 2013-2016.

10. W. C. W. Chan and S. Nie, Science, 1998, 281, 2016-2018.

11. X. Wu, H. Liu, J. Liu, K. N. Haley, J. A. Treadway, J. P. Larson, N. Ge, F. Peale and M. P. Bruchez, Nat. Biotechnol., 2003, 21, 41-46.

12. Q. Liu, B. Guo, Z. Rao, B. Zhang and J. R. Gong, Nano Lett., 2013, 13, $2436-2441$

13. L. A. Osminkina, K. P. Tamarov, A. P. Sviridov, R. A. Galkin, M. B. Gongalsky, V. V. Solovyev, A. A. Kudryavtsev and V. Y. Timoshenko, J. Biophotonics, 2012, 5, 529-535.

14. A. Burns, H. Ow and U. Wiesner, Chem. Soc. Rev., 2006, 35, 1028-1042.

15. H. Ow, D. R. Larson, M. Srivastava, B. A. Baird, W. W. Webb and U. Wiesner, Nano Lett., 2005, 5, 113-117.

16. W. Qin, K. Li, G. Feng, M. Li, Z. Yang, B. Liu and B. Z. Tang, Adv. Funct. Mater., 2013, 24, 635-643.

17. C. Wu and D. T. Chiu, Angew. Chem. Int. Ed., 2013, 52, 3086-3109.

18. N. C. Shaner, M. Z. Lin, M. R. McKeown, P. A. Steinbach, K. L. Hazelwood, M. W. Davidson and R. Y. Tsien, Nat. Methods, 2008, 5, $545-551$.

19. K. Li, W. Qin, D. Ding, N. Tomczak, J. Geng, R. Liu, J. Liu, X. Zhang, H. Liu, B. Liu and B. Z. Tang, Sci. Rep., 2013, 3, 1150. 
20. Q. Zhao, K. Li, S. Chen, A. Qin, D. Ding, S. Zhang, Y. Liu, B. Liu, J. Z. Sun and B. Z. Tang, J. Mater. Chem., 2012, 22, 15128-15135.

21. C. W. T. Leung, Y. Hong, S. Chen, E. Zhao, J. W. Y. Lam and B. Z. Tang, J. Am. Chem. Soc., 2012, 135, 62-65.

22. M. A. Woodruff and D. W. Hutmacher, Prog. Polym. Sci., 2010, 35, $1217-1256$

23. J.-S. Hu, Y. Guo, H.-P. Liang, L.-J. Wan and L. Jiang, J. Am. Chem. Soc., 2005, 127, 17090-17095.

24. M. Akbulut, P. Ginart, M. E. Gindy, C. Theriault, K. H. Chin, W. Soboyejo and R. K. Prud'homme, Adv. Funct. Mater., 2009, 19, 718-725. 25. X. Zhang, C. Dong, J. A. Zapien, S. Ismathullakhan, Z. Kang, J. Jie, X. Zhang, J. C. Chang, C.-S. Lee and S.-T. Lee, Angew. Chem. Int. Ed., $2009,48,9121-9123$.

26. F. Bai, Z. Sun, H. Wu, R. E. Haddad, E. N. Coker, J. Y. Huang, M. A. Rodriguez and H. Fan, Nano Lett., 2011, 11, 5196-5200.

27. L. Tang, X. Yang, Q. Yin, K. Cai, H. Wang, I. Chaudhury, C. Yao, Q. Zhou, M. Kwon, J. A. Hartman, I. T. Dobrucki, L. W. Dobrucki, L. B. Borst, S. Lezmi, W. G. Helferich, A. L. Ferguson, T. M. Fan and J. Cheng, PNAS, 2014, 111, 15344-15349.

28. R. F. Kubin and A. N. Fletcher, J. Lumin., 1982, 27, 455-462.

29. L. Z. Chen, J. M. Zheng, Y. Zhang, L. X. Yang, J. Q. Wang, J. Ni, D. X. Cui, C. Q. Yu and Z. L. Cai, Mol. Ther., 2011, 19, 1521-1528.

30. G. K. Wilson and Z. Stamataki, Int. J. Hepatol., 2012, 2012, 292591. 
31. T. Kato, M. Tanaka and M. Oba, Plos One, 2013, 8.

32. P. Bianco, X. Cao, P. S. Frenette, J. J. Mao, P. G. Robey, P. J. Simmons and C. Y. Wang, Nat. Med., 2013, 19, 35-42.

33. K. Ma, H. Shen, S. Shen, M. Xie, C. Mao, L. Qiu and Y. Jin, J. Gene Med., 2011, 13, 290-301.

34. S. V. Boddapati, G. G. M. D'Souza, S. Erdogan, V. P. Torchilin and V. Weissig, Nano Lett., 2008, 8, 2559-2563.

35. M. L. Barcellona and E. Gratton, Eur. Biophys. J., 1990, 17, 315-323.

36. G. Cosa, K. S. Focsaneanu, J. R. N. McLean, J. P. McNamee and J. C. Scaiano, Photochem. Photobiol., 2001, 73, 585-599.

37. Y. Gao, Y. Cui, J. K. Chan and C. Xu, Am. J. Nucl. Med. Mol. Imaging, $2013,3,232-246$. 


\section{Chapter 3 Highly fluorescent polycaprolactones}

\section{with tunable light emission wavelengths across}

\section{visible to NIR spectral window}

The previous chapter demonstrated a novel strategy of improving fluorescence in solid state and photostability by covalently bonding fluorescent dye in the middle of PCL. The fluorescent polymer designed by this method has good performance in long-term bioimaging. Moreover, PCL synthesized on fluorescent dyes makes it well biocompatibility and biodegradability. To further explore the potential applications of fluorescent polymer by this method, in this chapter, I extend a series of highly fluorescent and photostable PCL with tunable light absorption and emission wavelengths across visible to NIR spectral window. I aim to further use the fluorescent polymer with NIR emission for tissue scaffold. This work has been published in Advanced Material Interface in 2016.

\subsection{Introduction}

Polycaprolactone (PCL), as a member of the family of biodegradable polyesters, ${ }^{1}$ has attracted much attention from both basic research and pharmaceutical/medical industry. ${ }^{2}$ PCL has been approved by the United States Food and Drug Administration (FDA) for its increasing use in drug-delivery ${ }^{3,4}$ 
and tissue engineering ${ }^{5-7}$. The unique properties of PCL compared to other polymers such as PLGA include its slow degradation rate and mechanical properties that enables facile shaping and manufacturing. Particularly, PCL possesses superior rheological and viscoelastic properties over many other biodegradable polymers ${ }^{8}$. Recently, many multifunctional and well-organized PCL three-dimensional scaffolds with various shapes have been developed and applied in tissue engineering, such as bone engineering, cartilage engineering and skin engineering. ${ }^{9-12}$

The increasing use of PCL scaffolds in any type of tissue engineering makes it essential to study and understand their degradation behavior. Previous methods of tracking degradation of PCL scaffolds have been largely limited to measurement of molecular weight and mass loss as well as change of crystallinity and mechanical properties. ${ }^{13,14}$ At in vitro level, these methods are tedious and time-consuming. At in vivo level, these methods often require invasive retrieval of scaffolds from a body.

Recently, Artzi et al. ${ }^{15}$ and Kim et al. ${ }^{16}$ reported linear correlation between in vivo and in vitro erosion profiles in fluorescent dye-labelled PEG:dextran hydrogels and collagen hydrogels, which enables non-invasive tracking of erosion in biodegradable materials based on fluorescence imaging. Nevertheless, this non-invasive fluorescence imaging has never been used for tracking degradation of PCL scaffolds which are widely used in tissue engineering, to the best of our knowledge. It remains challenging to monitor noninvasively the degradation of implanted scaffolds using commercially available PCL which is 
not traceable via fluorescence. To that end, development of functional PCL polymers that are highly fluorescent in solid states are important. Furthermore, the fluorescence of PCL scaffolds offers the desired contrast to study the biotic/abiotic interface where the cells or tissues emit fluorescence distinguishable from that of scaffolds. ${ }^{17,} 18$

Previously reported molecular design, for example, PCL end-capped with small molecular fluorophores, still suffers from the intermolecular aggregation of the fluorophores and consequent fluorescence quenching in solid states. ${ }^{19-21}$ Recently, we reported a facile strategy of enhancing the fluorescence brightness and photostability by covalently linking an organic fluorophore, di(thiophene-2-yl)-diketopyrrolopyrrole (DPP), in the middle of PCL. ${ }^{22,} 23$ When the chain length of PCL is long enough, the polymer shows remarkably bright fluorescence in solid states and significantly enhanced photostability for cell imaging and long-term tracking. ${ }^{18}$ Our strategy of the molecular design toward PCL-dye-PCL polymers is effective to minimize the intermolecular aggregation of the dye, thus leading to bright and photostable fluorescence in solid states. In addition, the strong covalent bonding between the dye and PCL reduces potential early-stage leaching of dyes compared to physical blends of dyes/polymers.

In this chapter, I present molecular design, synthesis and processing of a series of highly fluorescent and photostable PCL with tunable light absorption and emission wavelengths across visible to NIR spectral window. In particular, a near-infrared (NIR) fluorescent PCL was designed and synthesized to enable 
deeper tissue penetration that is essential for in vivo applications, in which biological tissues have relatively low absorption and negligible autofluorescence in the NIR region. These tailor-made PCL polymers not only show strong fluorescence and robust photostability in solid states, but also exhibit good miscibility with PCL compared to physically blending organic dyes with commercial PCL. Moreover, these fluorescent polymers could be processed into nanoparticles stabilized by Pluronic 127 in water, resulting in well dispersible fluorescent ink for patterning at different length scales. Finally, the highly NIR-fluorescent PCL was used to monitor the degradation of PCL scaffolds via the evolution of the fluorescence intensity versus the mass loss of the scaffolds.

\subsection{Materials and Methods.}

\subsubsection{Materials and characterization}

All reagents and chemicals were purchased form Sigma Aldrich and used as received. All reactions were carried out under $\mathrm{N}_{2}$ atmosphere unless noted specifically. NMR spectra were recorded by Avance 300 NMR. Thermal gravimetric analysis (TGA) results were recorded by Pyris Diamond TGA. DSC results were recorded by Cryo DSC-Mettler Toledo 822e. Transmission electron microscope (TEM) measurements were carried out on a JEM-3010 (JEOL, Japan) electron microscope operating at an acceleration voltage of 120 $\mathrm{kV}$. UV-vis absorption spectra were recorded on a UV-2450 (SHIMADZU, 
Japan) spectrophotometer. Steady-state photoluminescence emission spectra were recorded with LS 55 (PerkinElmer, USA) fluorescence spectrometer. Fluorescent quantum yield was measured by Edinburgh fluorescence spectrometer FLS980. The particle size was measured with a dynamic light scattering (DLS) instrument, Zetasizer Nano ZS (Malvern, UK). The number-average molecular weight $\left(M_{\mathrm{n}}\right)$ and molecular weight distribution $\left(M_{\mathrm{w}} / M_{\mathrm{n}}\right)$ were measured by gel permeation chromatography (GPC) system (Agilent 1260, USA) equipped with waters 1260 pump, a Agilent 1260 refractive index detector, a UV-vis detector and a styragel ${ }^{\mathbb{B}}$ HT column. Tetrahydrofuran (THF) was used as the eluent $\left(1 \mathrm{~mL} \mathrm{~min}^{-1}\right)$, and polystyrene was used as the standard for calibration.

\subsubsection{Methods}

\section{The synthesis of 2,2'-(9,9-dioctyl-9H-fluorene-2,7-diyl)dithiophene (1)}

The mixture of 4,4,5,5-tetramethyl-2-(thiophen-2-yl)-1,3,2-dioxaborolane (525 $\mathrm{mg}, 2.5 \mathrm{mmol}$ ) and 2,7-dibromo-9,9-dioctyl-9H-fluorene (548 $\mathrm{mg}, 1 \mathrm{mmol}$ ) in toluene/ethanol/ $\mathrm{K}_{2} \mathrm{CO}_{3}(2 \mathrm{M})(4: 2: 1, \mathrm{vol}) 28 \mathrm{~mL}$ was bubbled with nitrogen for $15 \mathrm{~min}$. After the $3 \% \mathrm{Pd}\left(\mathrm{PPh}_{3}\right)_{4}(90 \mathrm{mg})$ was added, the solution was stirring and reflux for $12 \mathrm{~h}$. After being cooled down to room temperature, the mixture was poured into di-water and extracted by dichloromethane (DCM) twice. The crude product was further purified by column chromatography using hexane as eluent, and then $490 \mathrm{mg}$ faint yellow oil was obtained (yicld: $90 \%$ ). 


\section{The synthesis of 5,5'-(9,9-dioctyl-9H-fluorene-2,7-diyl)bis}

\section{(2- bromothiophene) (2)}

A solution of NBS $(2.4 \mathrm{mmol}, 409 \mathrm{mg})$ in THF $(20 \mathrm{ml})$ was added dropwise in dark to a suspension of Compound $1(1 \mathrm{mmol}, 555 \mathrm{mg})$ in dry THF $(20 \mathrm{~mL})$ at $-78{ }^{\circ} \mathrm{C}$. The mixture was stirred at $-78^{\circ} \mathrm{C}$ for $3 \mathrm{~h}$, and then allowed to room temperature. After stirring for $12 \mathrm{~h}$, the mixture was poured into di-water and extracted by DCM. The crude product was purified by column chromatography using hexane as eluent. The $510 \mathrm{mg}$ yellow oil was obtained (yield: $80 \%$ ). 

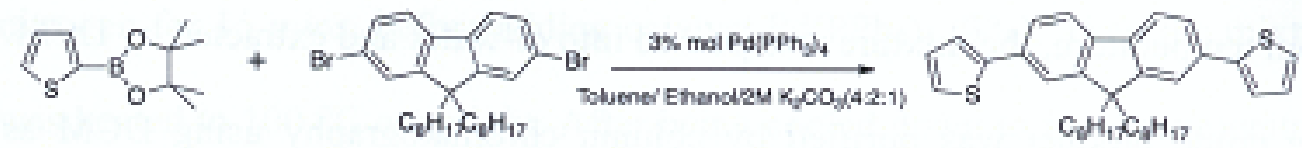

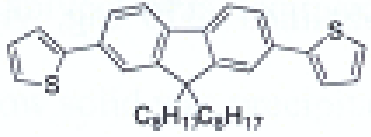

1

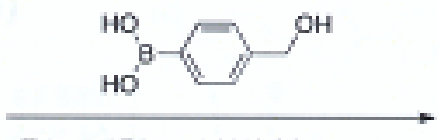

Toluenel EthanoV2M $\mathrm{K}_{2} \mathrm{CO}_{3}(4: 2: 1)$ $3 \%$ mol PdipPh $\mathrm{Pl}_{4}$

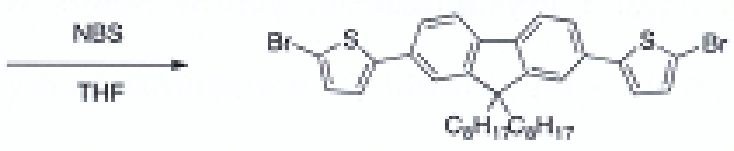

2

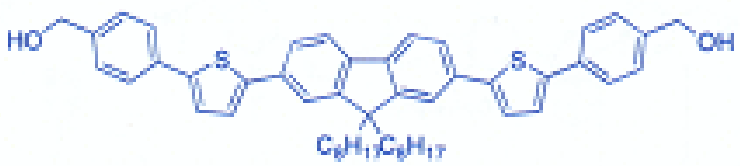

3
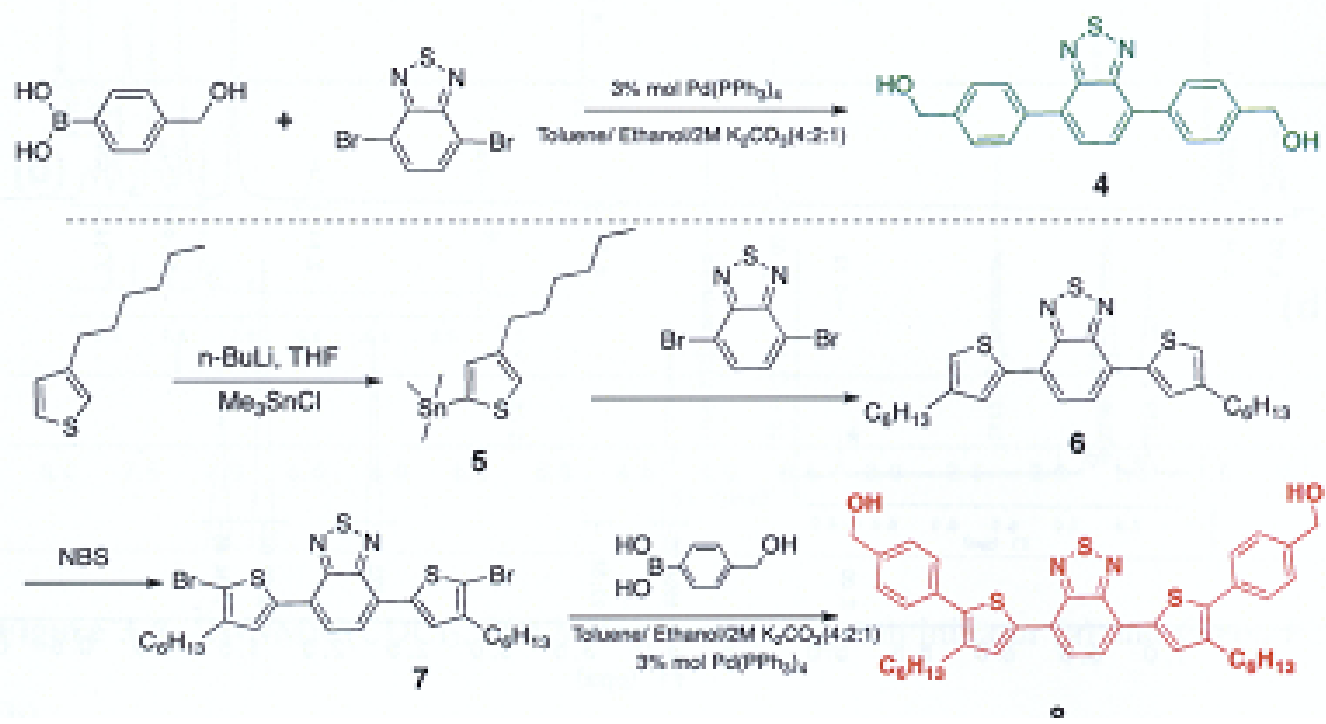

Scheme 3-1. Synthetic routes to blue-, green- and red-initiator with hydroxyl groups.

\section{The synthesis of DPTFT (3)}

To a mixture of compound $2 \quad(510 \quad \mathrm{mg}, \quad 0.71 \mathrm{mmol})$ and (4-(hydroxymethyl)phenyl)boronic acid (327 mg, $2.15 \mathrm{mmol}$ ) in toluene and ethanol (2:1, by vol), $\mathrm{K}_{2} \mathrm{CO}_{3}(2 \mathrm{M})$ solution was added. The mixture was bubbled under nitrogen for $15 \mathrm{mins}$, before adding catalyst $\mathrm{Pd}\left(\mathrm{PPh}_{3}\right)_{4}(3 \%, 23$ $\mathrm{mg}$ ). Then the mixture was heated to $100^{\circ} \mathrm{C}$ overnight. After cooled down to 
room temperature, the mixture was poured into di-water and extracted by DCM. The crude product was purified by column chromatography using DCM as eluent. After recrystallization, yellow solids were obtained $(370 \mathrm{mg}$, yield: $68 \%)$

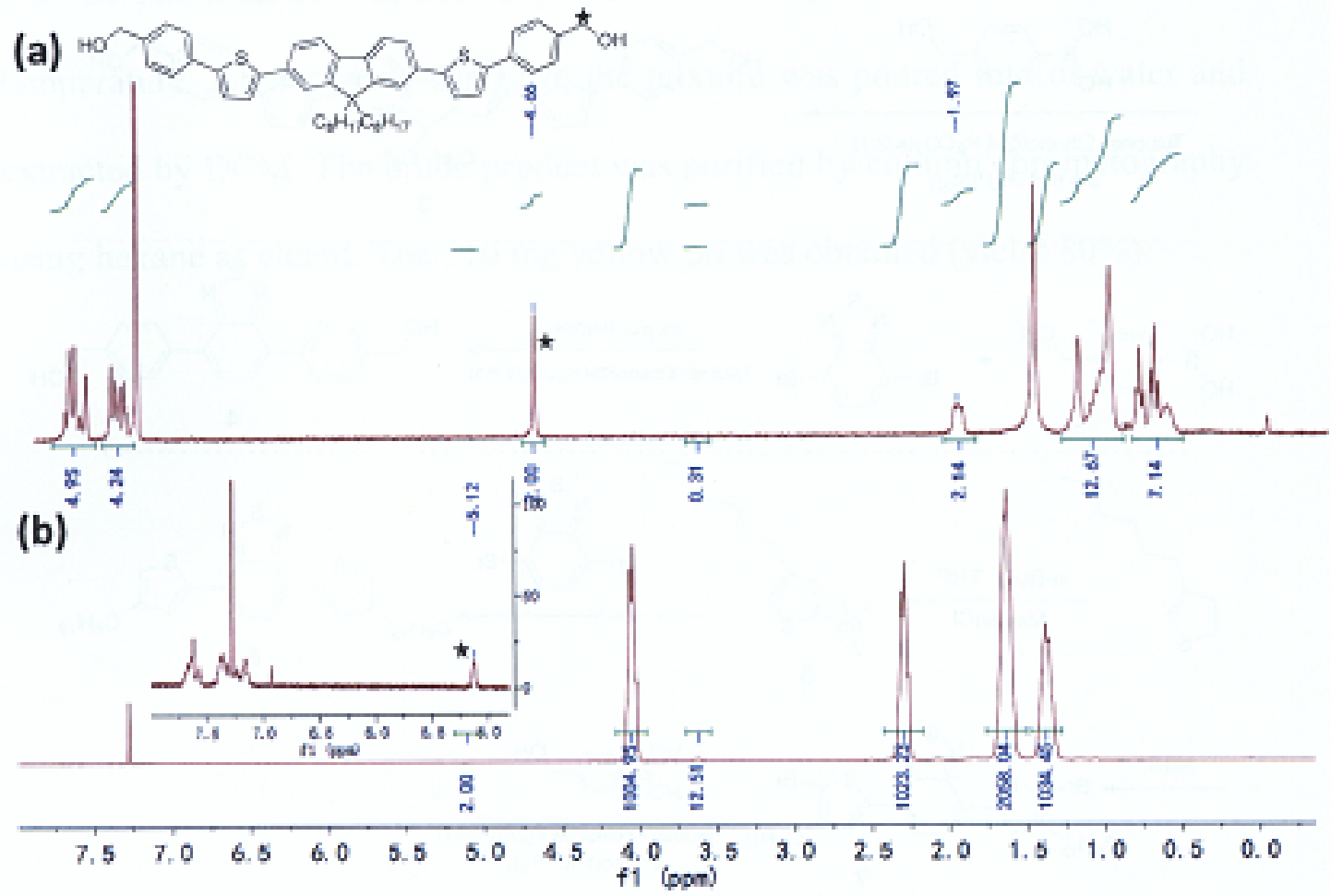

Figure 3-1. 'H-NMR $\left(\mathrm{CDCl}_{3}, 300 \mathrm{MHz}\right)$ spectra of blue initiator (a) and Blue-PCL (b).

\section{The synthesis of DPBT (4)}

To a mixture of 4,7-dibromobenzo[c][1,2,5]thiadiazole $(294 \mathrm{mg}, 1 \mathrm{mmol})$ and (4-(hydroxymethyl)phenyl)boronic acid (456 $\mathrm{mg}, 3 \mathrm{mmol}$ ) in toluene and ethanol (2:1), $\mathrm{K}_{2} \mathrm{CO}_{3}(2 \mathrm{M})$ solution was added. The mixture was bubbled under 
nitrogen for 15 mins, before adding catalyst $\mathrm{Pd}\left(\mathrm{PPh}_{3}\right)_{4}(3 \%)$. Then the mixture was heated to $100{ }^{\circ} \mathrm{C}$ overnight. After being cooled down to room temperature, the solvent was removed by rotary evaporator. After washed by water, the yellow solid was precipitated by recrystallization in chloroform (yield: $55 \%$ ).

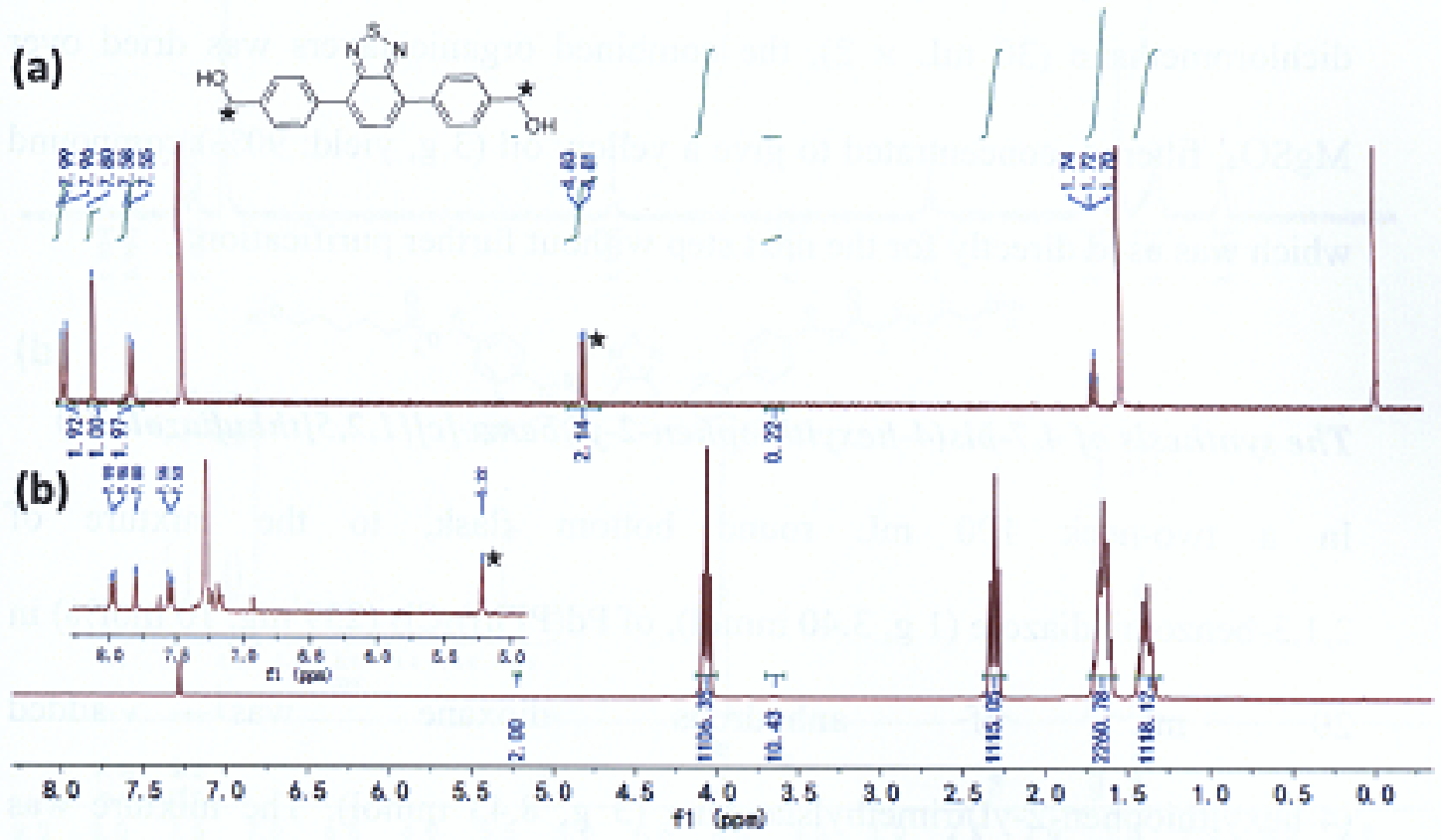

Figure 3-2. ${ }^{1} \mathrm{H}-\mathrm{NMR}\left(\mathrm{CDCl}_{3}, 300 \mathrm{MHz}\right)$ spectra of green initiator (a) and Green-PCL (b).

\section{The synthesis of (4-hexylthiophen-2-yl)trimethylstannane (5)}

In a two-neck $100 \mathrm{ml}$ round bottom flask, to the solution of 3-hexylthiophene (1.68 g, $10 \mathrm{mmol})$ in $50 \mathrm{~mL}$ of THF was added dropwise $n$-BuLi (12 mmol, 7.5 $\mathrm{mL}$ of $1.6 \mathrm{M}$ in $\mathrm{n}$-hexane) at $-78{ }^{\circ} \mathrm{C}$ under $\mathrm{Ar}$ atmosphere. The resulting mixture was stirred for $1 \mathrm{~h}$ at the same temperature and then allowed to warm to 
$0{ }^{\circ} \mathrm{C}$ and stirred at this temperature for $30 \mathrm{~min}$, and again cooled to $-78{ }^{\circ} \mathrm{C}$, followed by slow addition of $\mathrm{Me}_{3} \mathrm{SnCl}(12 \mathrm{mmol}, 12 \mathrm{~mL}$ of $1.0 \mathrm{M}$ in $\mathrm{n}$-hexane). The resulting solution was slowly warmed to room temperature and stirred overnight. The reaction was quenched with water and extracted with dichloromethane $(30 \mathrm{~mL} \times 2)$, the combined organic layers was dried over $\mathrm{MgSO}_{4}$, filtered, concentrated to give a yellow oil (3 g, yield: $90 \%$ ) compound which was used directly for the next step without further purification.

\section{The synthesis of 4,7-bis(4-hexylthiophen-2-yl)benzo[c][1,2,5]thiadiazole (6)}

In a two-neck $100 \mathrm{~mL}$ round bottom flask, to the mixture of 2,1,3-benzothiadiazole (1 $\mathrm{g}, 3.40 \mathrm{mmol})$, of $\mathrm{Pd}\left(\mathrm{PPh}_{3}\right)_{2} \mathrm{Cl}_{2}(239 \mathrm{mg}, 10 \mathrm{~mol} \%)$ in $20 \mathrm{~mL}$ of anhydrous dioxane was added (4-hexylthiophen-2-yl)trimethylstannane ( $3 \mathrm{~g}, 8.43 \mathrm{mmol})$. The mixture was bubbled with Argon for 10 min and refluxed for 20 hours. Then the solvent was removed under reduced pressure, and the crude product was purified by column chromatography, by using hexane as the eluent to isolate the target compound as a orange solid (1.5 g, yield: $94 \%)$.

\section{The synthesis of Compound 7}

A solution of NBS $(2.4 \mathrm{mmol}, 409 \mathrm{mg})$ in THF $(20 \mathrm{~mL})$ was added dropwise in dark to a suspension of 4,7-bis(4-hexylthiophen-2-yl)benzo[c][1,2,5]thiadiazole $(1 \mathrm{mmol}, 468.74 \mathrm{mg})$ in dry THF $(20 \mathrm{~mL})$ at $-78^{\circ} \mathrm{C}$. The mixture was stirred at $-78{ }^{\circ} \mathrm{C}$ for $3 \mathrm{~h}$, and then allowed to room temperature. Stirring was continued 
for $12 \mathrm{~h}$, the solvent was removed. After recrystallization from THF and methanol, $500 \mathrm{mg}$ (yield: $79 \%$ ) of red solid was obtained.

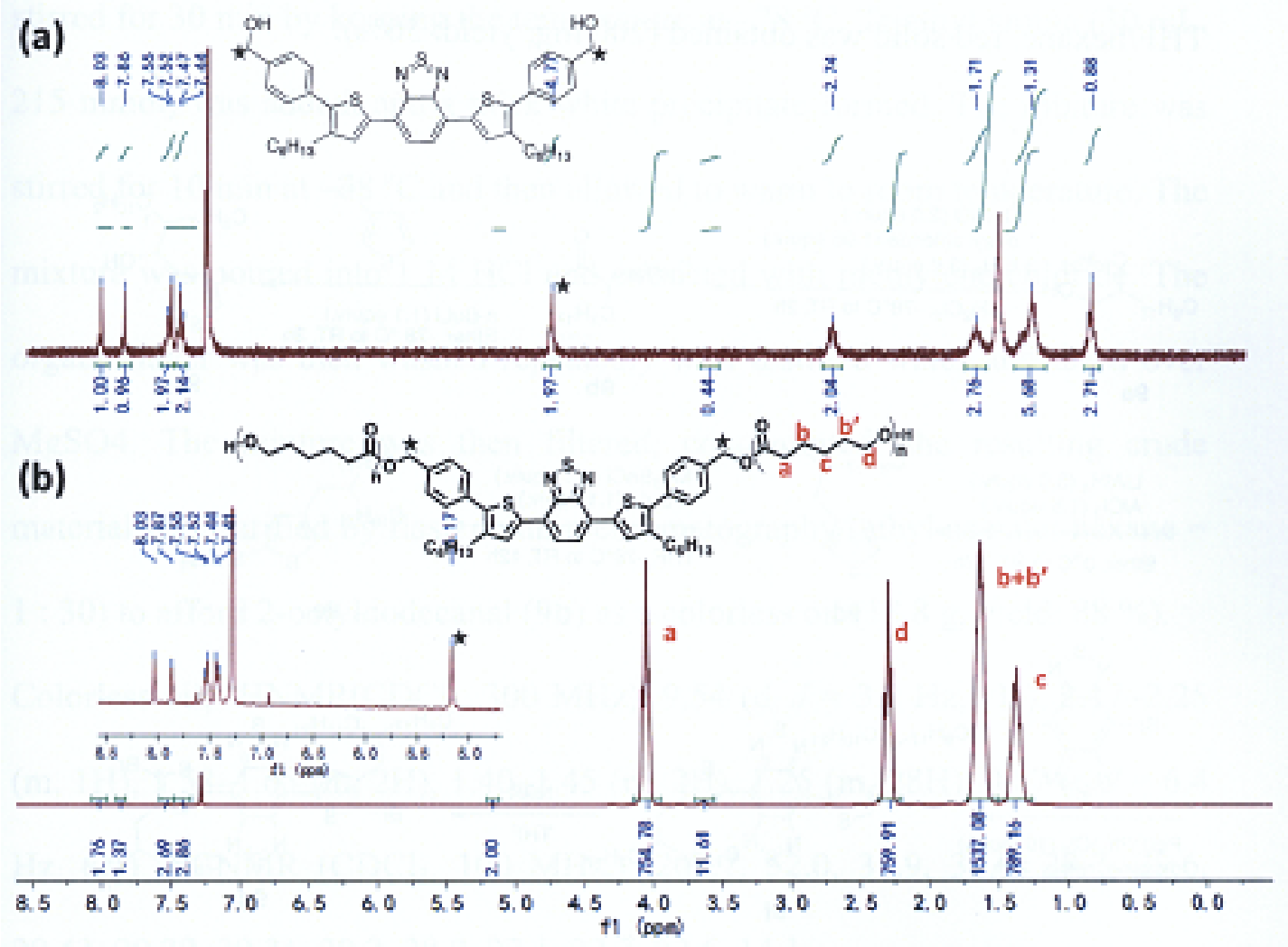

Figure 3-3. ${ }^{1} \mathrm{H}-\mathrm{NMR}\left(\mathrm{CDCl}_{3}, 300 \mathrm{MHz}\right)$ spectrum of red initiator (a) and Red-PCL (b).

\section{The synthesis of DPTBT compound (8)}

To a mixture of compound 7 (600 $\mathrm{mg}, 1 \mathrm{mmol})$ and (4-(hydroxymethyl)phenyl) boronic acid (380 mg, $2.5 \mathrm{mmol})$ in toluene and ethanol (2:1), add $\mathrm{K}_{2} \mathrm{CO}_{3}(2 \mathrm{M})$ solution. The mixture was bubbled under nitrogen for $15 \mathrm{mins}$, before adding catalyst $\mathrm{Pd}\left(\mathrm{PPh}_{3}\right)_{4}(3 \%, 90 \mathrm{mg})$. Then the mixture was heated to $100{ }^{\circ} \mathrm{C}$ overnight. After cooled down to room temperature, the mixture was poured into 
water and extracted with DCM. The crude product was purified by column chromatography using hexane/THF=2:1 (vol). After recrystallization in THF/hexane, red solid was obtained (200 mg, yield: $30 \%)$.

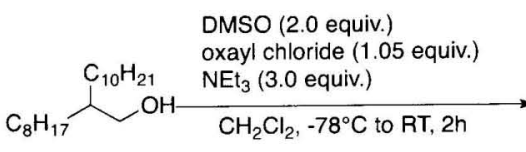

$9 a$
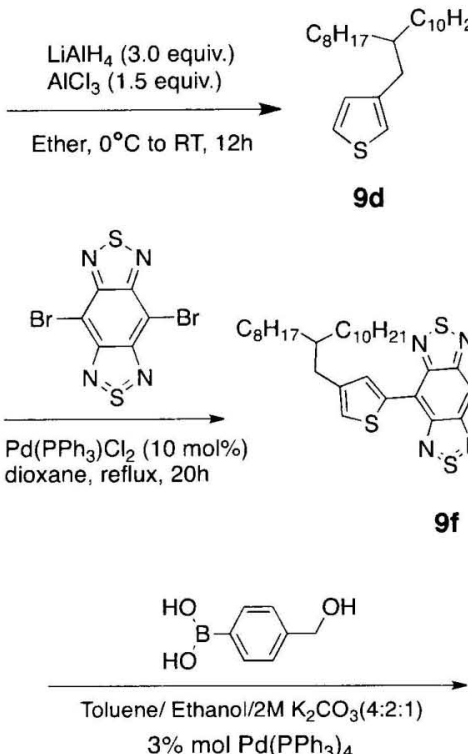

dioxane, reflux, $20 \mathrm{~h}$

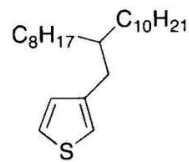

9d
91<smiles>[13CH3][13CH2]C([18OH])C=O</smiles>

$9 b$

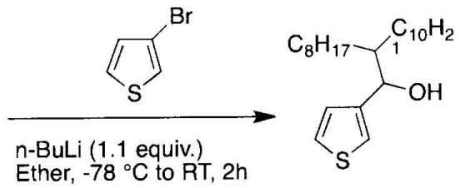

9c

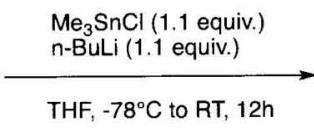

$\mathrm{THF},-78^{\circ} \mathrm{C}$ to $\mathrm{RT}, 12 \mathrm{~h}$

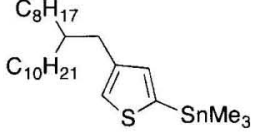

$9 e$

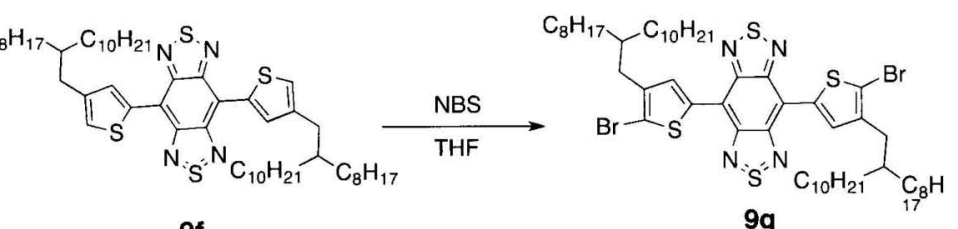

$9 \mathrm{~g}$

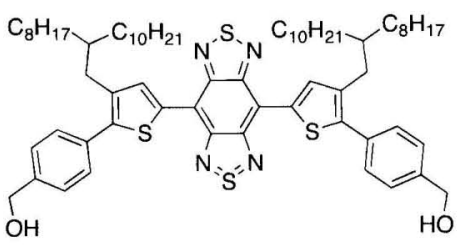

9

Scheme 3-2. The synthesis route to NIR initiator (DPBBT)

\section{Synthesis and characterization of 2-octyldodecanal (9b)}

In a two-neck $500 \mathrm{~mL}$ round bottom flask, to a solution of dimethyl sulfoxide $(10.2 \mathrm{~mL}, 143.6 \mathrm{mmol})$ in anhydrous methylene chloride $(350 \mathrm{~mL})$ was dropwise added oxalyl chloride $(6.48 \mathrm{~mL}, 75.6 \mathrm{mmol})$ at $-78{ }^{\circ} \mathrm{C}$ under $\mathrm{N}_{2}$ 
atmospherc. The mixture was stirred for $30 \mathrm{~min}$, and then 2-octyldodecan-1-ol (9a) $(21.5 \mathrm{~g}, 71.99 \mathrm{mmol})$ was added dropwise at $-78^{\circ} \mathrm{C}$ and the mixture was stirred for $30 \mathrm{~min}$ by keeping the temperature at $-78^{\circ} \mathrm{C}$. Triethyl amine $(30 \mathrm{~mL}$, $215 \mathrm{mmol}$ ) was added, and a thick white precipitate formed. The mixture was stirred for $10 \mathrm{~min}$ at $-78^{\circ} \mathrm{C}$ and then allowed to warm to room temperature. The mixture was poured into $1 \mathrm{M} \mathrm{HCl}$ and extracted with methylene chloride. The organic layer was then washed repeatedly with distilled water and dried over MgSO4. The mixture was then filtered, concentrated, the resulting crude matcrial was purified by flash column chromatography (ethyl acetate: hexane = $1: 30)$ to afford 2-octyldodecanal $(9 \mathbf{b})$ as a colorless oil (18.8 $\mathrm{g}$, yield: $88 \%$ ).

Colorless oil; ${ }^{1} \mathrm{HNMR}\left(\mathrm{CDCl}_{3}, 300 \mathrm{MHz}\right): 9.54(\mathrm{~d}, J=3.2 \mathrm{~Hz}, 1 \mathrm{H}), 2.17-2.25$ $(\mathrm{m}, 1 \mathrm{H}), 1.58-1.65(\mathrm{~m}, 2 \mathrm{H}), 1.40-1.45(\mathrm{~m}, 2 \mathrm{H}), 1.25(\mathrm{~m}, 28 \mathrm{H}), 0.87(\mathrm{t}, J=6.4$ $\mathrm{Hz}, 6 \mathrm{H}) .{ }^{13} \mathrm{CNMR}\left(\mathrm{CDCl}_{3}, 100 \mathrm{MHz},\right): 205.7,52.0,31.9,31.8,29.7,29.6$, $29.43,29.39,29.31,29.2,28.9,27.1,22.7,22.6,14.1$.

\section{Synthesis and characterization of 2-octyl-1-(thiophen-3-yl)dodecan-1-ol (9c)}

In a two-neck $250 \mathrm{~mL}$ round bottom flask, to a solution of 3-bromothiophene $(8.02 \mathrm{~g}, 49 \mathrm{mmol})$ in $100 \mathrm{~mL}$ anhydrous diethyl ether, $n$-BuLi $(33.7 \mathrm{~mL}, 53.95$ mmol, 1.64 $\mathrm{M}$ in hexane) was added dropwise at $-78^{\circ} \mathrm{C}$ under $\mathrm{N}_{2}$ atmosphere. After the addition was finished, the resulting mixture was stirred another 2 hours and then 2-octyldodecanal $(16.0 \mathrm{~g}, 53.95 \mathrm{mmol})$ was added quickly. The final mixture was allowed to warm to room temperature and was stirred for 2 hours. This reaction then was quenched with $60 \mathrm{~mL}$ water and extracted with 
ethyl acetate $(3 \times 50 \mathrm{ml})$. The combined organic layers were dricd over anhydrous $\mathrm{MgSO} 4$, filtered, concentrated under reduced pressure gives the crude product, which was purified by column chromatography on silica by using ethyl acetate/hexane $=1 / 50$ as the eluent to afford the product $9 \mathrm{c}$ as a colorless oil (18.3 g, yield: $98 \%$ ).

Colorless oil; ${ }^{1} \mathrm{H}$ NMR (400 MHz, $\mathrm{CDCl}_{3}$ ) 7.25-7.29 (m, 1H), $7.13(\mathrm{~d}, J=2.8$ $\mathrm{Hz}, 1 \mathrm{H}), 7.01(\mathrm{dd}, J=4.8,1.2 \mathrm{~Hz}, 1 \mathrm{H}), 4.75-4.78(\mathrm{~m}, 1 \mathrm{H}), 1.75(\mathrm{~m}, 1 \mathrm{H}), 1.64-$ $1.68(\mathrm{~m}, 1 \mathrm{H}), \quad 1.24(\mathrm{br}, 31 \mathrm{H}), 0.86-0.89(\mathrm{~m}, 6 \mathrm{H}) ;{ }^{13} \mathrm{C} \mathrm{NMR}\left(100 \mathrm{MHz}, \mathrm{CDCl}_{3}\right)$ $145.5,125.9,125.6,120.8,72.9,44.6,31.9,30.03,29.97,29.63,29.58,29.55$, $29.34,29.31,29.29,28.9,27.1,27.0,22.7,14.1$.

\section{Synthesis and characterization of 3-(2-octyldodecyl)thiophene (9d)}

In a two-neck $250 \mathrm{~mL}$ round bottom flask, to a suspension of $\mathrm{LiAlH}_{4}(2.48 \mathrm{~g}$, $65.4 \mathrm{mmol}$ ) in $80 \mathrm{~mL}$ anhydrous diethyl ether was portionwise added $\mathrm{AlCl}_{3}$ (4.36 g, $\quad 32.7 \mathrm{mmol}$ ) at $0 \quad{ }^{\circ} \mathrm{C}$ under $\mathrm{N}_{2}$ atmosphere. Then, 2-octyl-1-(thiophen-3-yl)dodecan-1-ol (3) (8.3 g, $21.8 \mathrm{mmol})$ was added dropwise to the mixture at $0{ }^{\circ} \mathrm{C}$, and then stirred at room temperature overnight. This reaction was quenched with water $(20 \mathrm{~mL})$ at $0{ }^{\circ} \mathrm{C}$ by slowly adding water to the mixture. After that the mixture was filtered through celite, the residue was washed with diethyl ether $(50 \mathrm{ml})$ two times, then the organic phase was separatcd, dried over anhydrous $\mathrm{MgSO}_{4}$, filtered and distillation of solvent under reduced pressure gives the crude products, which was purified by column chromatography by using hexane as the eluent. The compound $9 \mathrm{~d}$ was obtained 
as colorless oil ( $4.2 \mathrm{~g}, 53 \%$, with $36 \%$ recovery of compound $9 \mathrm{c})$.

${ }^{1} \mathrm{H}$ NMR $\left(300 \mathrm{MHz}, \mathrm{CDCl}_{3}\right) 7.22(\mathrm{~m}, 1 \mathrm{H}), 6.89(\mathrm{~m}, 2 \mathrm{H}), 2.56(\mathrm{~d}, J=6.6 \mathrm{~Hz}$, 2H), $1.59(\mathrm{~m}, 1 \mathrm{H}), 1.25(\mathrm{br} \mathrm{s}, 32 \mathrm{H}), 0.88(\mathrm{t}, J=6.6 \mathrm{~Hz}, 6 \mathrm{H}) ;{ }^{13} \mathrm{C}$ NMR $(75$ $\left.\mathrm{MHz}, \mathrm{CDCl}_{3}\right) 141.92,128.82,124.77,120.64,38.96,34.73,33.35,31.96$, $30.04,29.71,29.69,29.67 .29 .39,29.38,26.65,22.72,14.15$.

\section{Synthesis and characterization of}

\section{trimethyl(4-(2-octyldodecyl)thiophen-2-yl)stannane (9e)}

In a two-neck $100 \mathrm{~mL}$ round bottom flask, to the solution of $9 \mathrm{~d}(3.92 \mathrm{~g}, 10.75$ $\mathrm{mmol})$ in $50 \mathrm{~mL}$ of THF was dropwise added $n$-BuLi $(11.8 \mathrm{mmol}, 4.72 \mathrm{~mL}$ of $2.5 \mathrm{M}$ in hexane) at $-78^{\circ} \mathrm{C}$ under $\mathrm{Ar}$ atmosphere. The resulting mixture was stirred for $1 \mathrm{~h}$ at the same temperature and then allowed to warm to $0{ }^{\circ} \mathrm{C}$ and stirred at this temperature for $30 \mathrm{~min}$, and again cooled to $-78^{\circ} \mathrm{C}$, followed by slow addition of $\mathrm{Me}_{3} \mathrm{SnCl}(11.8 \mathrm{mmol}, 11.8 \mathrm{~mL}$ of $1.0 \mathrm{M}$ in $\mathrm{n}$-hexane). The resulting solution was slowly warmed to room temperature and stirred overnight. The reaction was quenched with water and extracted with dichloromethane $(30 \mathrm{~mL} \times 2)$, the combined organic layers was dried over $\mathrm{MgSO}_{4}$, filtered, concentrated to give a yellow oil (5.2 g, yield: $92 \%$ ) compound which was used directly for the next step without further purification.

${ }^{1} \mathrm{H}$ NMR (300 MHz, CDCl3) $7.15(\mathrm{~s}, 1 \mathrm{H}), 6.95$ (s, 1H), 2.58 (d, $\left.J=6.9 \mathrm{~Hz}, 2 \mathrm{H}\right)$, $1.59-1.64(\mathrm{~m}, 1 \mathrm{H}), 1.25(\mathrm{br} \mathrm{s}, 32 \mathrm{H}), 0.86-0.90(\mathrm{~m}, 6 \mathrm{H}), 0.35(\mathrm{~s}, 9 \mathrm{H})$. 


\section{Synthesis and characterization of Compound $9 f$}

In a two-neck $100 \mathrm{~mL}$ round bottom flask, to the mixture of 4,8-dibromo-BBT (0.14 g, $0.40 \mathrm{mmol})$, of $\mathrm{Pd}\left(\mathrm{PPh}_{3}\right)_{2} \mathrm{Cl}_{2}(28 \mathrm{mg} 10 \mathrm{~mol} \%)$ in $8 \mathrm{~mL}$ of anhydrous dioxane was added trimethyl(4-(2-octyldodecyl)thiophen-2-yl)stannane (9e) $(0.53 \mathrm{~g}, 1.0 \mathrm{mmol})$. The mixture was bubbled with Argon for $10 \mathrm{~min}$ and refluxed for 20 hours. Then the solvent was removed under reduced pressure, and the crude product was purified by column chromatography, by using hexane as the eluent to remove the unreacted tin compound, using DCM/hexane $=5 / 95$ to isolate the compound $9 \mathrm{f}$ as a dark green solid (230 mg, yield: $63 \%$ ) ${ }^{1} \mathrm{H}$ NMR (300 MHz, $\left.\mathrm{CDCl}_{3}\right): 8.68$ (s, 2H), 7.22 (s, 2H), 2.70 (d, $J=6.6 \mathrm{~Hz}$, $4 \mathrm{H}), 1.77$ (br s, 2H), $1.30(\mathrm{~m}, 64 \mathrm{H}), 0.87(\mathrm{~m}, 12 \mathrm{H}) ;{ }^{13} \mathrm{C} \mathrm{NMR}(75 \mathrm{MHz}$, $\left.\mathrm{CDCl}_{3}\right): 150.79,142.74,137.05,134.58,127.11,113.28,39.01,34.98,33.43$, $31.97,31.96,30.17,29.79,29.76,29.71,29.44,29.40,26.73,22.72,14.14$.

\section{Synthesis and characterization of Compound $9 g$}

In a two-neck $250 \mathrm{~mL}$ round bottom flask, to a solution of compound 7 (225 $\mathrm{mg}, 0.245 \mathrm{mmol})$ in $\mathrm{CHCl}_{3}(65 \mathrm{~mL})$ and acetic acid $(65 \mathrm{~mL})$ was slowly added NBS (96 mg, $0.539 \mathrm{mmol})$ in $\mathrm{CHCl}_{3}(5 \mathrm{~mL})$ and acetic acid $(5 \mathrm{~mL})$ over $30 \mathrm{~min}$ at room temperature under argon atmosphere and exclusion of light. The mixture was stirred for one hour at room temperature and was then the solvent was removed by reduced pressure to give a crude solid product which was purified by column chromatography on silica by using DCM/hexane $=1 / 100$ to afford $96 \mathrm{mg}$ (yield: 36\%) desired product as a green solid. 
${ }^{1} \mathrm{H}$ NMR $\left(300 \mathrm{MHz}, \mathrm{CDCl}_{3}\right): 8.25(\mathrm{~s}, 2 \mathrm{H}), 2.54(\mathrm{~d}, J=6.9 \mathrm{~Hz}, 4 \mathrm{H}), 1.78$ (br s, 2H), 1.25-1.37 (m, 64H), 0.85-0.89 (m, 12H);

${ }^{13} \mathrm{C}$ NMR (75 MHz, $\left.\mathrm{CDCl}_{3}\right): 149.85,141.96,136.75,133.77,118.00,111.69$, $38.59,34.08,33.4,31.98,31.95,30.22,29.81,29.78,29.73,29.47,29.41,26.61$, $22.72,22.70,14.13$

\section{The synthesis of DPTBBT (9)}

To a mixture of compound $9 \mathrm{~g} \quad\left(\begin{array}{lllll}66.8 & \mathrm{mg}, & 0.062 & \mathrm{mmol}) & \text { and }\end{array}\right.$ (4-(hydroxymethyl)phenyl)boronic acid $(29 \mathrm{mg}, 0.186 \mathrm{mmol})$ in toluene and ethanol (2:1), add $\mathrm{K}_{2} \mathrm{CO}_{3}(2 \mathrm{M})$ solution. The mixture was bubbled under nitrogen for $15 \mathrm{mins}$, before adding catalyst $\mathrm{Pd}\left(\mathrm{PPh}_{3}\right)_{4}(3 \%, 7 \mathrm{mg})$. Then the mixture was heated to $100^{\circ} \mathrm{C}$ overnight. After cooled down to room temperature, the mixture was poured into water and extracted with DCM twice. It was removed solvent under rotary evaporator. It runs gel column using hexane: dichloromethane $(1: 1)$ as eluent. The $41 \mathrm{mg}$ green solid was recrystallized from THF and ethanol (yield: 60\%). 
(a)

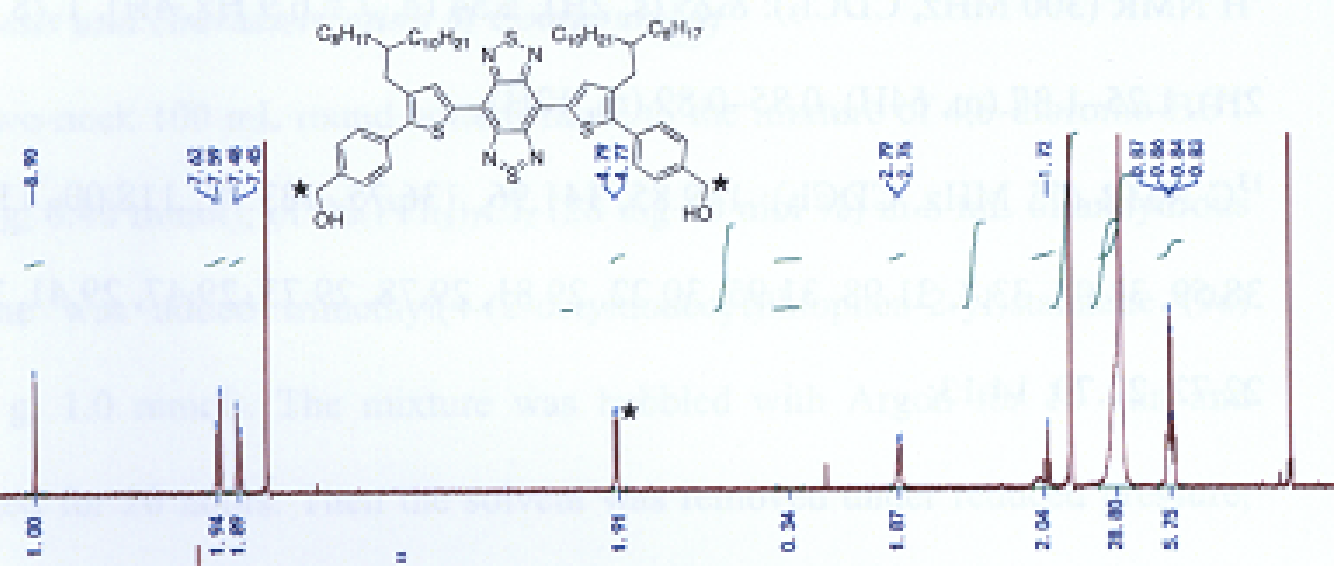

(b)

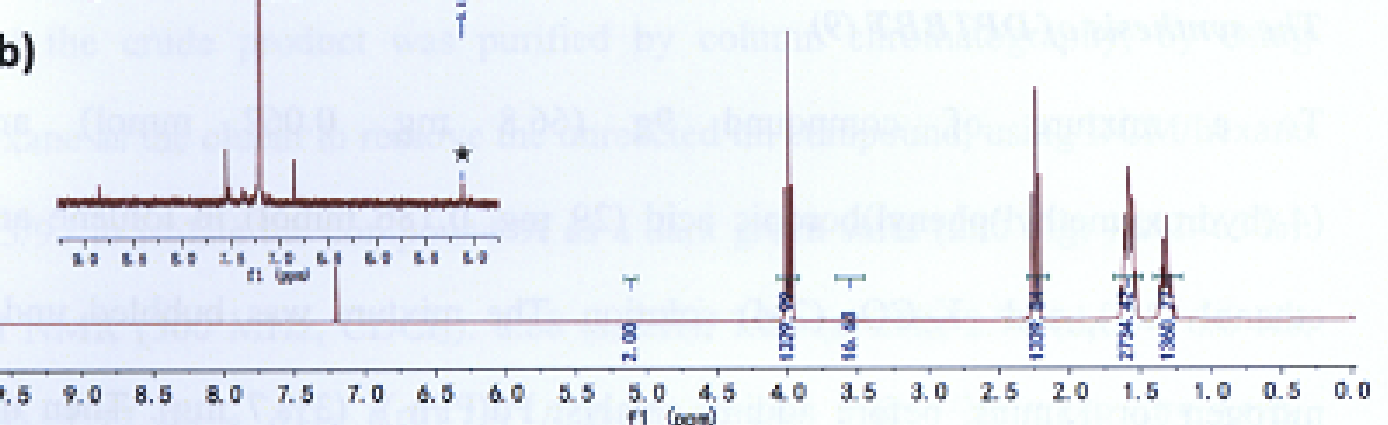

Figure 3-4. ${ }^{1} \mathrm{H}-\mathrm{NMR}\left(\mathrm{CDCl}_{3}, 300 \mathrm{MHz}\right)$ spectra of NIR initiator (a) and NIR-PCL (b).

\section{General synthetic procedure for PCL-dye-PCL}

The initiator in the Schlenk tube was degassed and refilled nitrogen twice. The monomer caprolactone and catalyst was injected by syringe and heated to 110 ${ }^{\circ} \mathrm{C}$. The mixture cannot stir any more after reacting over $12 \mathrm{~h}$. A small mount of THF was added to dissolve polymer, and then the polymer products were purified by precipitation in methanol twice and diethyl ether once. 

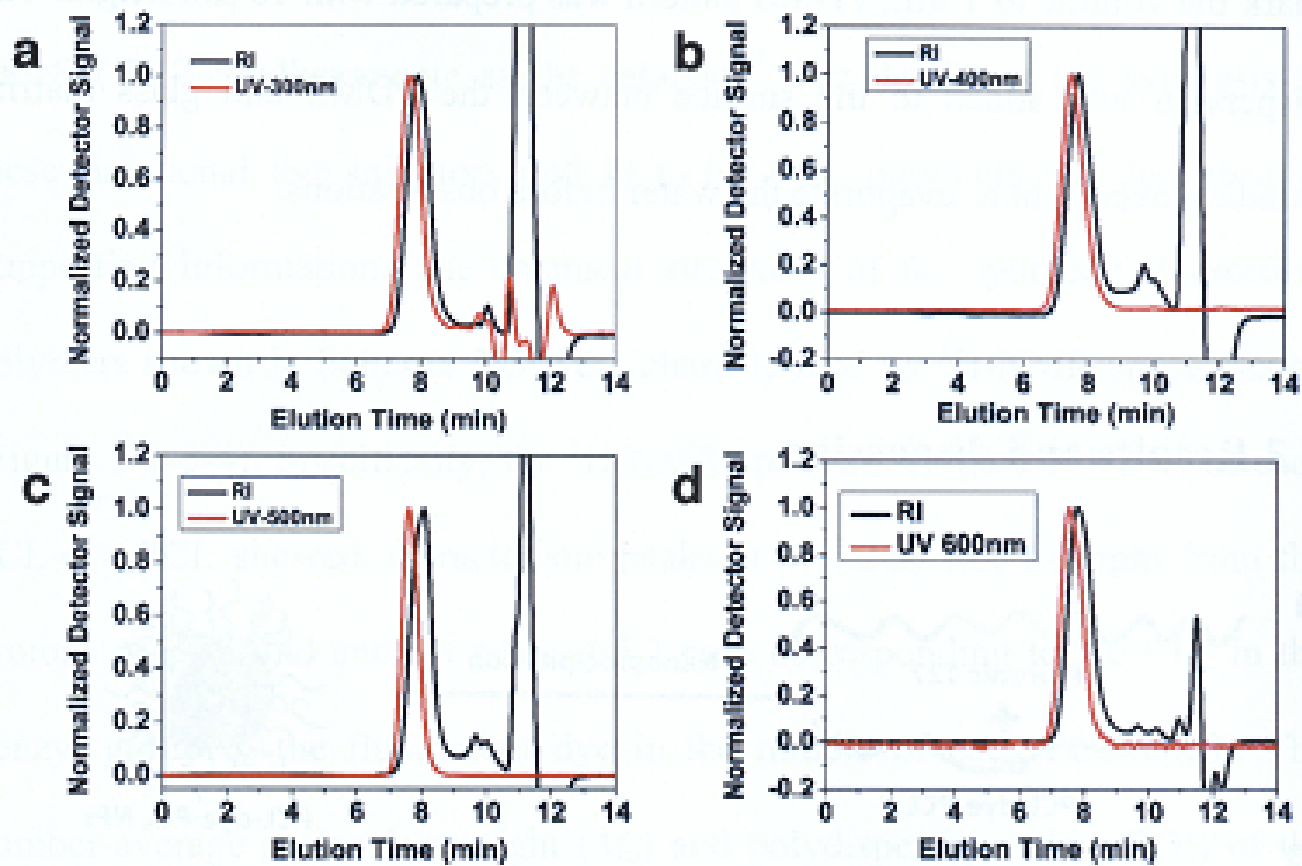

Figure 3-5. GPC traces of Blue-PCL, Green-PCL, Red-PCL and NIR-PCL running in THF as the eluent.

\section{Preparation of fluorescent polymer NPs}

$20 \mathrm{~mL}$ of di-water was added dropwise (over $10 \mathrm{~min}$ ) into a mixture of fluorescent PCL (50 mg) and Pluronic $127(160 \mathrm{mg})$ in $4 \mathrm{~mL}$ of dioxane under sonication. Then the dispersion was dialyzed by $4 \mathrm{KDa}$ membrane against di-water over $48 \mathrm{~h}$ to remove organic solvent. The dispersion was stored in fridge as stock dispersion.

\section{Micro-contact patterning}

$10 \mathrm{~mL}$ of stock dispersion of the NPs prepared above was centrifuged at 10000 rpm for $10 \mathrm{~min}$. The upper solution was removed and di-water was added to 
mark the volume to $1 \mathrm{~mL}$. PDMS pattern was prepared with $10 \mu \mathrm{m}$ height. The dispersion was added to the surface between the PDMS and glass matrix, standing overnight to evaporate the water before observations.

\subsection{Results and discussion}

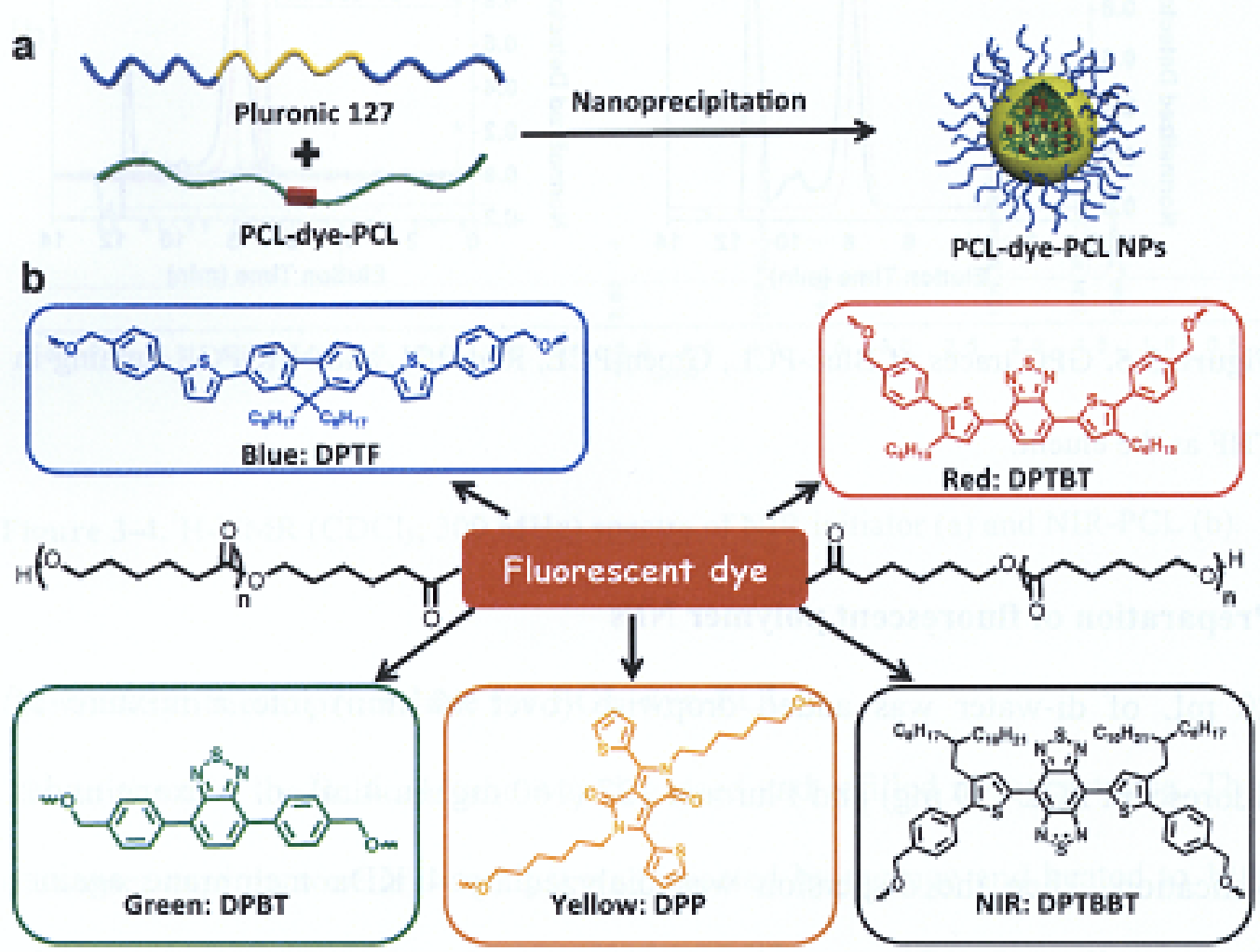

Scheme 3-3. Schematic illustration of preparation (a) of PCL-dye-PCL NPs and the chemical structures (b) of fluorophores with various emission wavelengths.

\section{Synthesis and characterization of PCL-dye-PCL}

Fluorescent polymers PCL-dye-PCL with different emission wavelengths were synthesized by ring-opening polymerization of $\varepsilon$-caprolactone from 
different fluorophore derivatives bearing double $-\mathrm{OH}$ groups as the initiators and $\mathrm{Sn}$ (II) 2-ethylhexanoate as the catalyst. ${ }^{23}$ The details of the synthesis of these functional dye initiators and PCL-dye-PCL polymers are described in Supporting Information. The chemical structures of the synthetic fluorescent polymers shown in Scheme 3-3 were charactcrized by ${ }^{1}$ H-NMR spectroscopy (Figure 3-1-3-4). Specifically, the ${ }^{1}$ H-NMR spectrum (Figure 3-1-3-4) of each PCL-dyc-PCL showed characteristic peaks at 4.1, 2.3, 1.6, $1.4 \mathrm{ppm}$ from the protons of PCL and another peak at $5.2 \mathrm{ppm}$ corresponding to the $\mathrm{CH}_{2}$ in the benzyl group of the fluorescent dye in the middle of cach PCL chain. The number-average molecular weight $\left(M_{\mathrm{n}}\right)$ and polydispersity index (PDI) of the obtained polymers were measured with gel permeation chromatography (GPC) (Table 3-1, Figure 3-5). Most of these fluorescent PCL showed a $M_{\mathrm{n}}$ around 2 $\times 10^{4} \mathrm{~g} / \mathrm{mol}$ and a relativcly narrow PDI $(1.5 \sim 2.0)$. In addition, $M_{\mathrm{n}}$ estimated from the end-group analysis of the ${ }^{1} \mathrm{H}-\mathrm{NMR}$ spectrum was consistent with that measured by GPC (Table 3-1). The relatively narrow PDI and the good overlapping between the GPC traces recorded with refractive-index (RI) and UV-vis detectors indicate the well-defined structures of these polymers, each of which consists of an organic dye in the middle of the PCL chain.

The thermal properties of different PCL-dye-PCL polymers were examined by differential scanning calorimetry (DSC) and thermogravimetric analysis (TGA). While all of these polymers showed a similar melting point in the range of $58-60{ }^{\circ} \mathrm{C}$, thermal decomposition temperature varied in the range of $240-310^{\circ} \mathrm{C}$ depending on the molecular weight of each polymer (Table 3-1, 
Figures 3-6-3-7).

Table 3-1. Summary of PCL-dyc-PCL characterizations.

\begin{tabular}{|c|c|c|c|c|c|c|c|c|c|}
\hline Polymer & $M_{n}\left({ }^{*} 10^{3}\right)^{2}$ & $M_{n} / M_{n}$ & $\mathrm{n}^{4}$ & $n^{b}$ & $\begin{array}{c}T_{\mathrm{d}} \\
\left(\mathrm{C}^{\circ} \mathrm{C}\right)\end{array}$ & $T_{\text {(in }}$ & $\underset{(n m)^{c}}{\lambda_{c}}$ & $\begin{array}{l}\lambda_{\text {max.cue }} \\
(\mathrm{nmm})^{\mathrm{c}}\end{array}$ & $\begin{array}{c}\lambda_{\text {murem,usad }} \\
(\mathrm{nm})\end{array}$ \\
\hline Blue-PCL & 18 & 1.5 & 78 & 80 & 250 & 59 & 389 & 430,458 & 434,458 \\
\hline Green-PCL & 23 & 1.6 & 100 & 110 & 260 & 60 & 390 & 492 & 489 \\
\hline Yellow-PCL & 38 & 2.0 & 185 & 198 & 306 & 58 & 510,550 & 558,602 & 564,604 \\
\hline Red-PCL & 16 & 1.5 & 70 & 69 & 245 & 59 & 491 & 593 & 594 \\
\hline NIR-PCL & 18 & 1.7 & 78 & 80 & 265 & 59 & 788 & 942 & 986 \\
\hline
\end{tabular}

a) Molecule weight was calculated from GPC running in THF as eluent and linear poly(styrene) homopolymers as standards. b) Molecule weight was calculated from end-group analysis of $1 \mathrm{H}-\mathrm{NMR} . \mathrm{c})$ Measured in THF solutions.
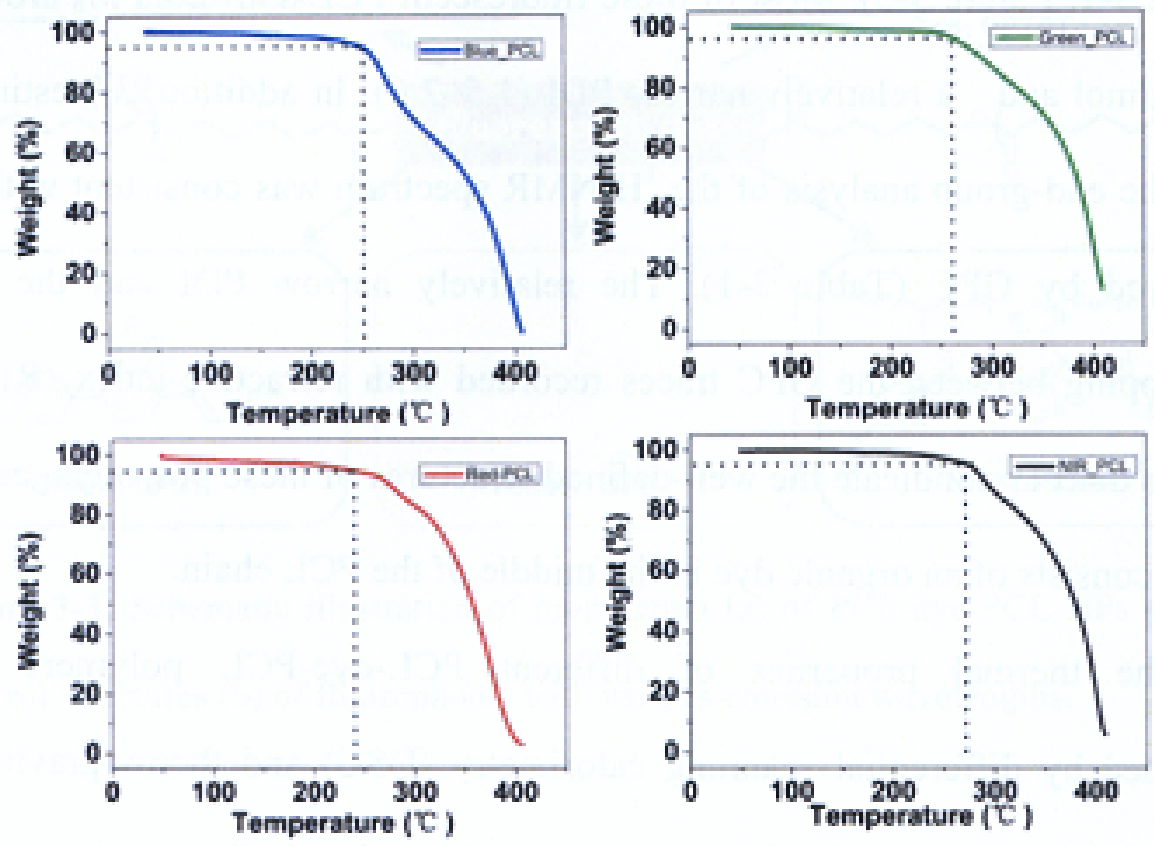

Figure 3-6. TGA results of Blue-PCL, Green-PCL, Red-PCL and NIR-PCL. 

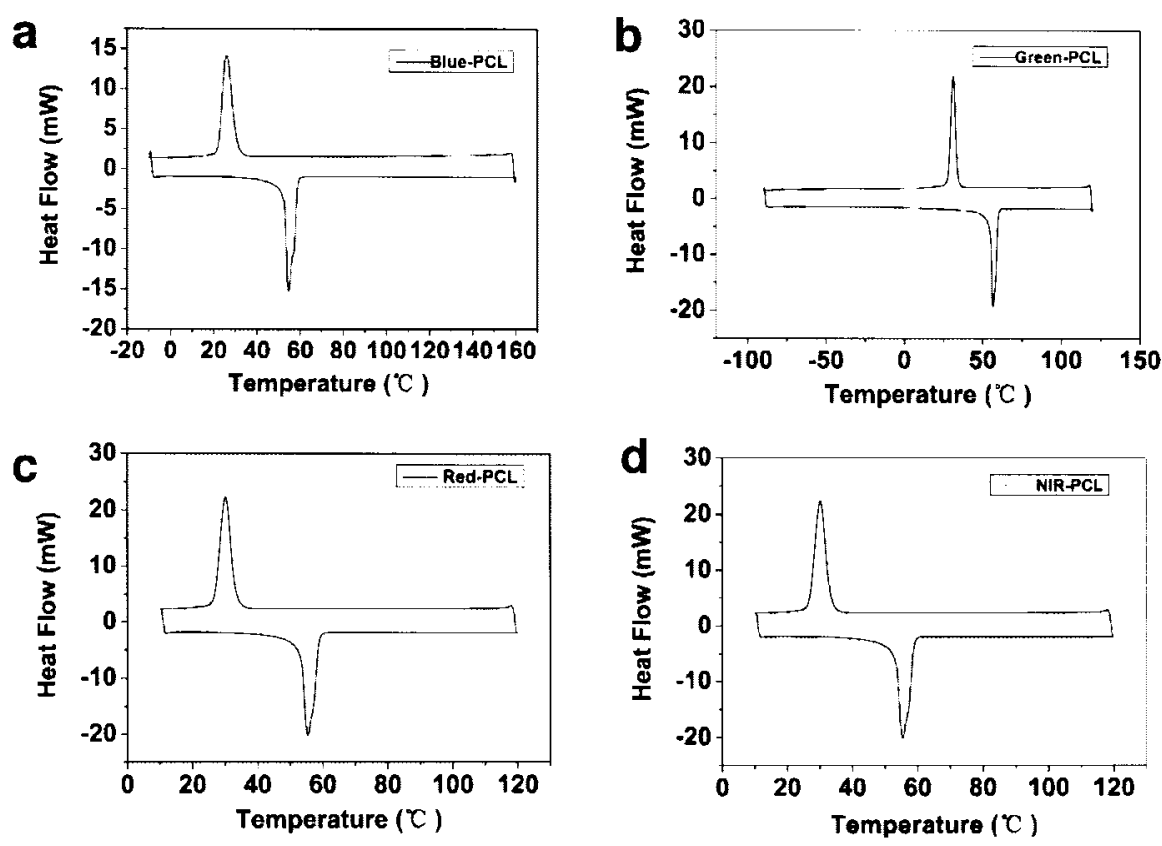

Figure 3-7. DSC results of Blue-PCL (a), Green-PCL (b), Red-PCL (c) and NIR-PCL (d).

\section{Optical properties}

The optical properties of PCL-dye-PCL polymers are presented in Figure 3-8. The UV-VIS-NIR absorption spectra in THF (Figure 3-8) show the maximum absorption peaks at 389, 390, 491 and $788 \mathrm{~nm}$ for Blue-, Green-, Red- and NIR-PCL, respectively. While the emission spectrum of Blue-PCL shows a vibronic double peak at 430 and $458 \mathrm{~nm}$, respectively, the other fluorescent PCL polymers (including Green-, Red- and NIR-PCL) all show a broad featureless emission band centered at 492, 593 and $942 \mathrm{~nm}$, respectively, largely due to the strong intramolecular charge transfer.

Similar to Yellow-PCL reported in our previous publications, ${ }^{22,23}$ all of the other fluorescent PCL polymers exhibited strong fluorescence in solid states 
including bulk powders and solid films. Moreover, there was no significant change between the emission spectra of these polymers in films and those in dilute solutions such as in THF (Figure 3-8), which implies that the PCL chains grafted from the organic dyes effectively suppress the aggregation-caused FL quenching caused by intermolecular $\pi-\pi$ stacking such as $J$-aggregation. The slight red-shift of the emission peak in NIR-PCL in solid states $v$. in dilute THF solution implies stronger $\pi-\pi$ interaction of BBT units compared to other organic dyes in the solid states. Particularly, the NIR-PCL shows a relative high FL quantum yield $(\Phi=0.01)$ in solid states compared to commercial NIR dyes such as IR 1048 ( $\Phi=0.004$ in dichloromethane), which enables the NIR-PCL as a promising fluorescent contrast agent for in vivo bioimaging.
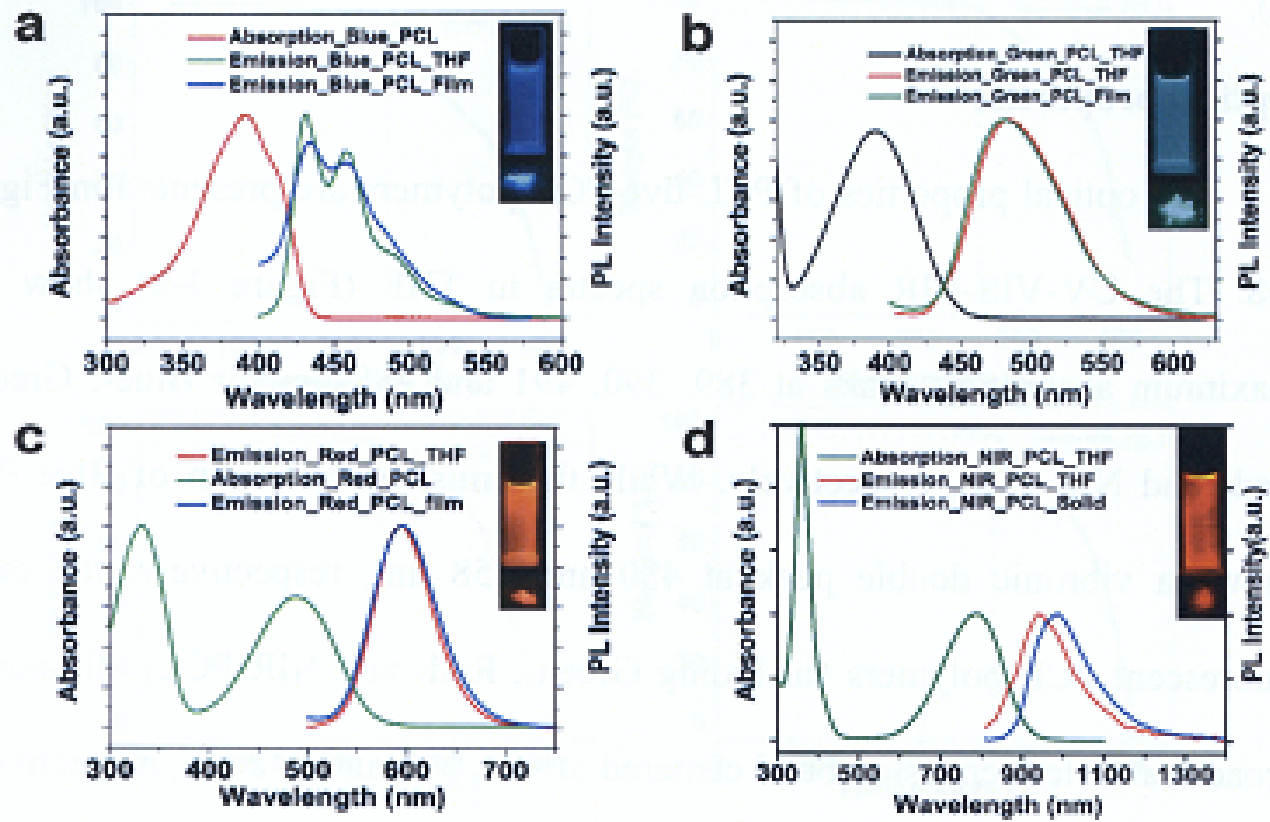

Figure 3-8. Absorption and emission spectra of Blue-PCL (a), Green-PCL (b), Red-PCL (c) and NIR-PCL (d) in THF and in films. Excitation wavelengths are 390, 
390, 490 and $790 \mathrm{~nm}$, respectively. The inset digital photographs show fluorescence images of these polymers both in solution and in solid powders under UV light (365 $\mathrm{nm})(\mathrm{a}-\mathrm{c})$, or $808 \mathrm{~nm}$ laser (d) irradiation.

\section{Colloidal PCL-dye-PCL nanoparticles as fluorescent ink for patterning}

To render the hydrophobic PCL-dye-PCL polymers described above dispersible into water, they were encapsulated into colloidal nanoparticles (NPs) via a nanoprecipitation ${ }^{23,24}$ process in the presence of Pluronic 127 as the stabilizer. The morphology and the size of the prepared NPs were characterized by scanning electron microscope (SEM) and dynamic light scattering (DLS) (Figure. 3-10). The average diameter measured from the SEM images of the spherical NPs, which was $63 \pm 11 \mathrm{~nm}$ for Blue-PCL, $54 \pm 10 \mathrm{~nm}$ for Green-PCL and $57 \pm 14 \mathrm{~nm}$ for Red-PCL, was similar for different colored PCL but generally smaller than that of the NPs measured by DLS in hydrated states. Moreover, these NPs composed of PCL-dye-PCL in the cores are highly fluorescent and can be used as water-borne ink for patterning such as macro-scale stamping (Figure. 3-9a), brush writing (Figure. 3-9b-c) and micro-contact printing (Figure. 3-9d-f) at different length scales. These fluorescent patterns maintained their features and brightness after being stored for at least one year under ambient conditions. 

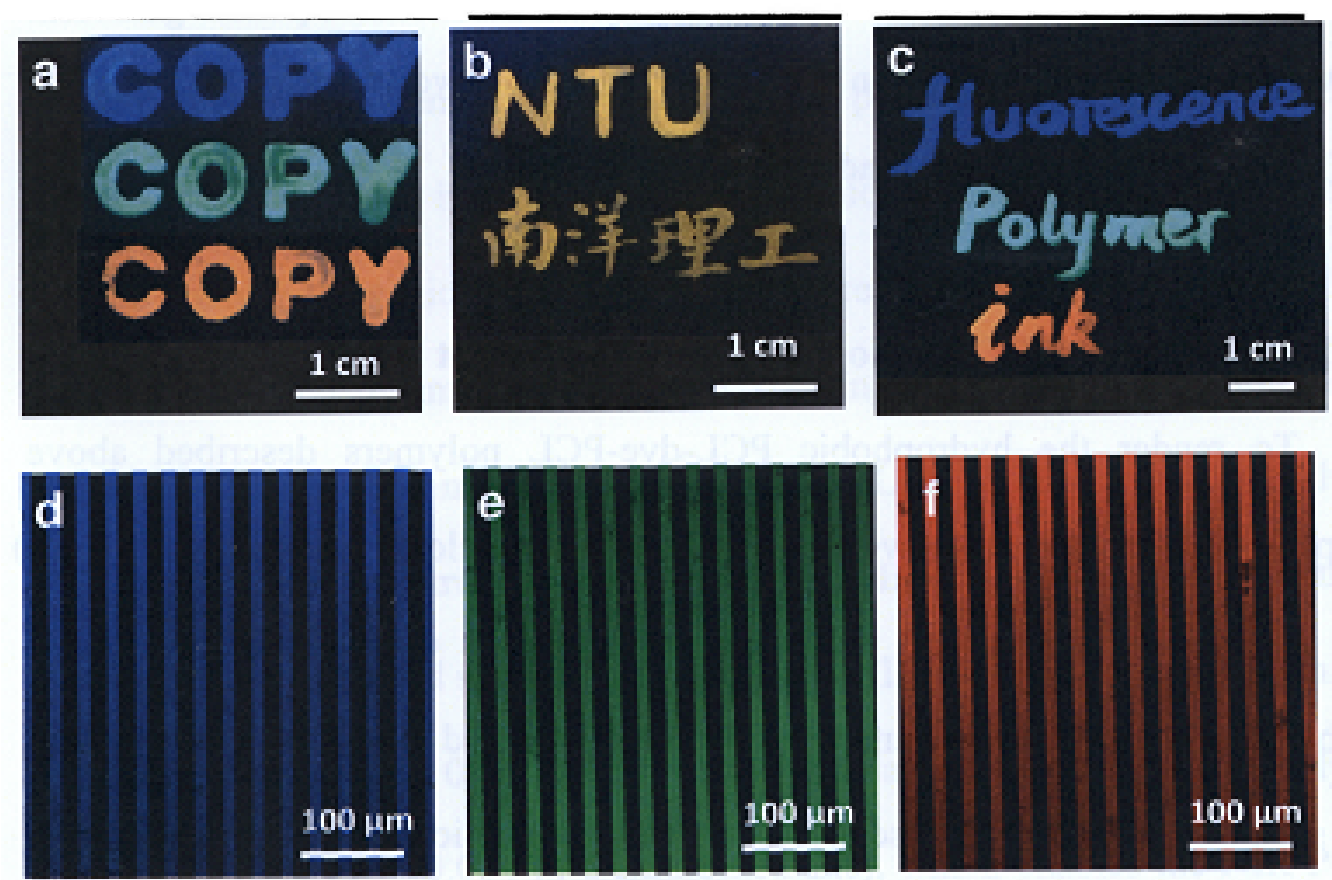

Figure 3-9. (a-c) Fluorescence images of PCL-dye-PCL patterns under UV light 365 $\mathrm{nm}$. The patterns were prepared by stamping (a) and brush writing (b and c) the colloidal NPs as water-borne ink on filter paper. (d-f) Confocal laser scanning microscope images of patterns prepared by micro-contact printing of colloidal NPs made of Blue- (d), Green- (e) and Red-PCL (f) on glass substrates.
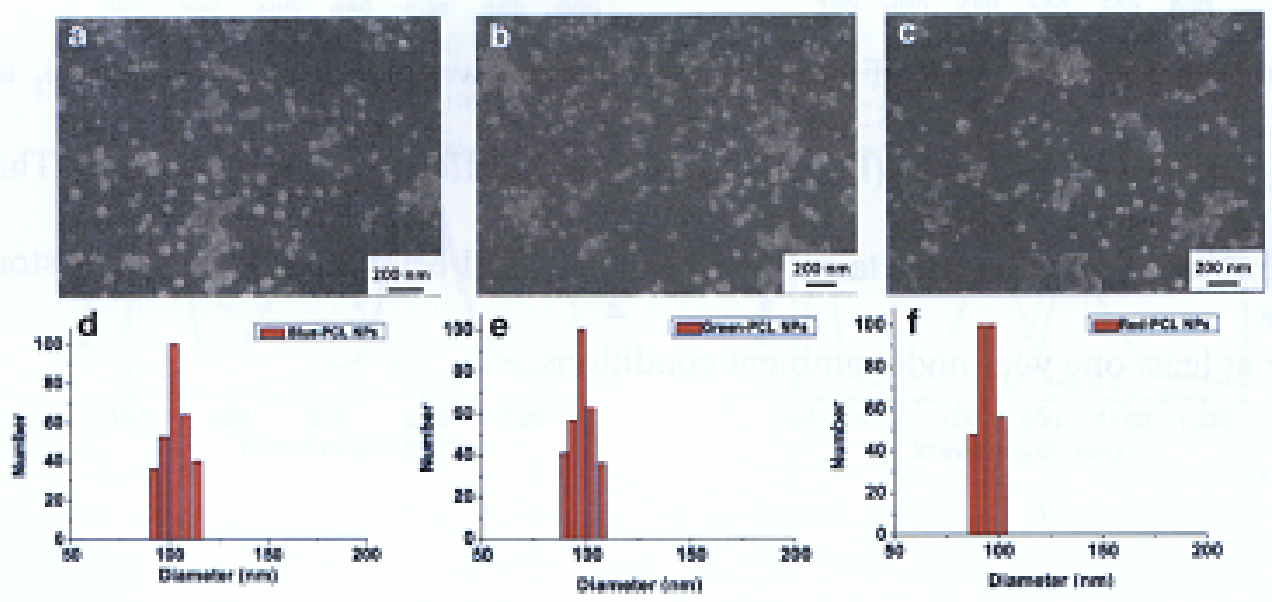
Figure 3-10. The SEM images (a-c) and DLS histograms (d-f) of Blue-PCL NPs (a and d), Green-PCL NPs (b and e) and Red-PCL NPs (c and f).
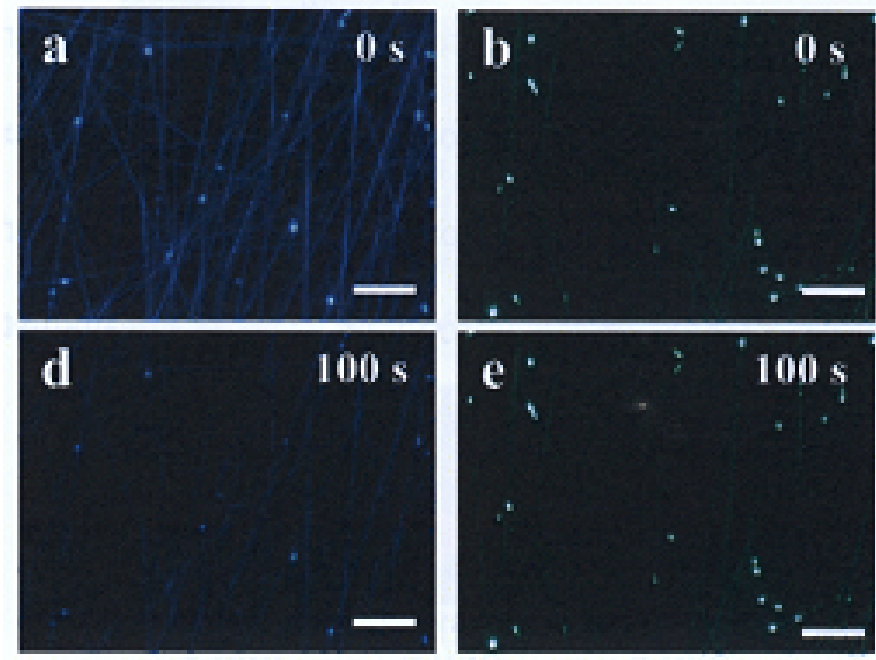

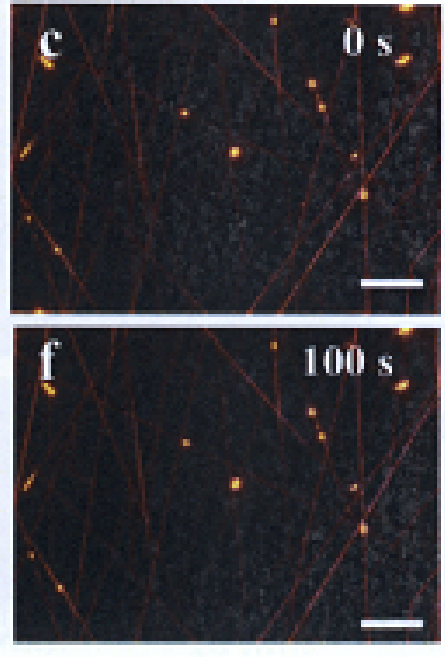

Figure 3-11. Photostability of nanofibers of Blue-PCL (a and d), Green-PCL (b and e) and Red-PCL (c and f) under fluorescence microscope for $100 \mathrm{~s}$ [scale bar: $100 \mu \mathrm{m}$ ]. (The excitation wavelength was $405 \mathrm{~nm}$ for Blue- and Green-PCL, and $514 \mathrm{~nm}$ for Red-PCL)

\section{Fluorescent nanofibers of PCL-dye-PCL and photostability}

To investigate the effect of the chemical structure of the organic dye on the fluorescence properties of PCL-dye-PCL in solid states, we further fabricated fluorescent nanofibers by electrospinning PCL-dye-PCL $(10 \% \mathrm{w} / \mathrm{w})$ mixed with commercial PCL in trifluoroethanol. All of the nanofibers (Figure 3-11) appeared more uniform in diameter (ca. $2 \mu \mathrm{m}$ ) than the physically blended mixture of commercial PCL and the dye initator under the fluorescence 
microscope. In addition, the nanofibers exhibited good photostability, maitaining their high fluorescence intensity after $100 \mathrm{~s}$ of light irradiation (50 W Mercury light source) under a fluorescence microscope. The relatively lower photostability of blue-PCL nanofibers compared to the green-PCL and the red-PCL nanofibers could be due to the shorter PCL chains in the former and/or the higher susceptibility of the blue fluorophore (DPTF) against photobleaching.

\section{The penetration depth of NIR fluorescence imaging}

To examine the application of NIR-PCL in fluorescence-based biological imaging and the penetration depth, we acquired NIR fluorescence signal from a model of \#-like scaffold prepared by blending NIR-PCL $(50 \%, \mathrm{w} / \mathrm{w})$ and commercial PCL $\left(M_{n}=70000 \mathrm{~g} / \mathrm{mol}\right)$. The scaffold was embeded inside two pieces of pork tissues (Figure 3-12a). The imaging setup is shown in Figure 3-15. The fluorescence images (Figure 3-12b) of the NIR-PCL scaffold show strong fluorescence signal at various thicknesses of the tissues, indicating deep penetration of tissues up to $10 \mathrm{~mm}$. The shape of the NIR-PCL scaffold appeared blurry with increase of the tissue thickness, mainly caused by light scattering from tissues. Nevethless, the fluorescence signal was strong enough for in vivo imaging applications. 


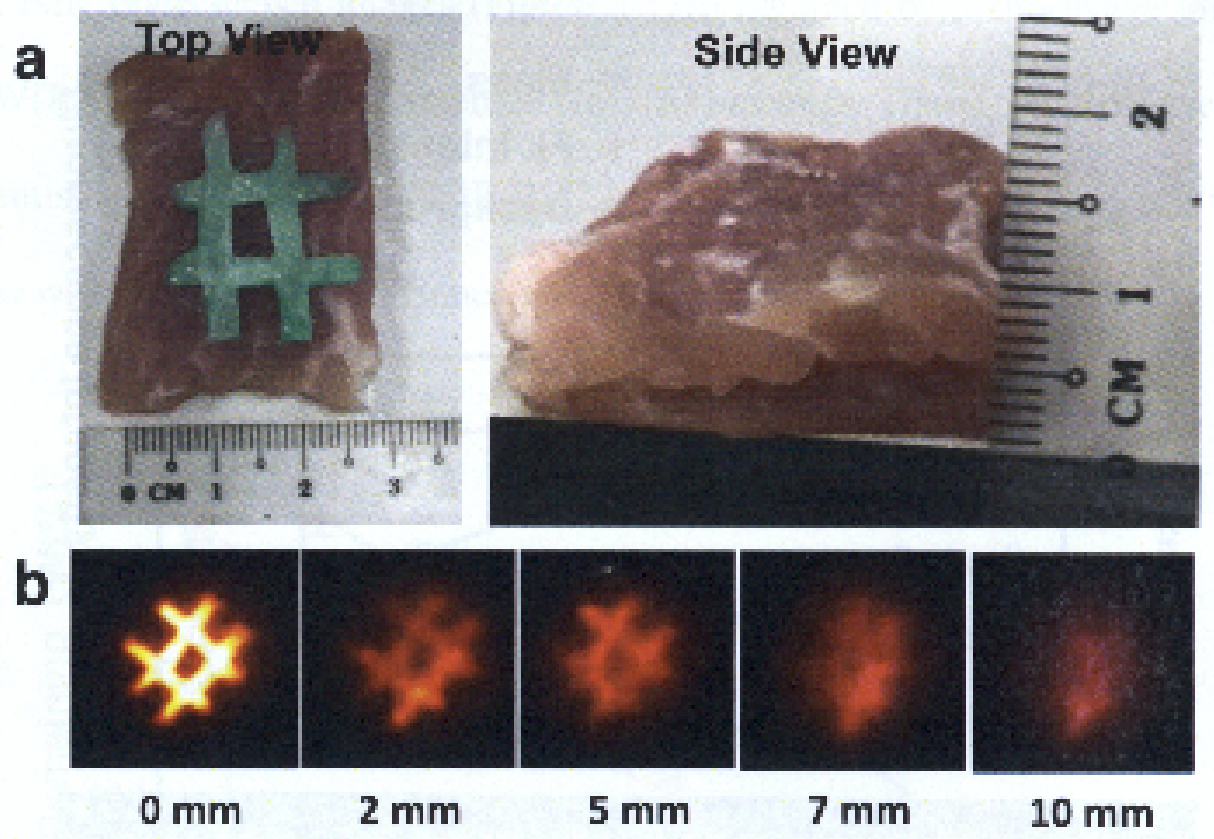

Figure 3-12. (a) Digital photographs of the \#-like NIR-PCL scaffold on the top of a piece of pork tissue (top view, left) and sandwiched between two pieces of pork tissues (side view, right). (b) The fluorescence images of the NIR-PCL scaffold embeded inside two pieces of pork tissues with various thicknesses.

\section{Quantifying in vitro degradation of NIR-PCL scaffolds}

To investigate the application of NIR-PCL for monitoring the degradation of the scaffolds non-invasively, we monitored the rate of the scaffold degradation by measuring changes of NIR fluorescence signals from the scaffold using a NIR camera. Specifically, a prepared star-like tetrapod scaffold made of NIR-PCL was soaked in aqueous solution of sodium hydroxide $(\mathrm{NaOH})$ at various concentrations. For comparison, we also measured the mass loss of the same scaffold during the degradation process. Figure 3-14 shows the 

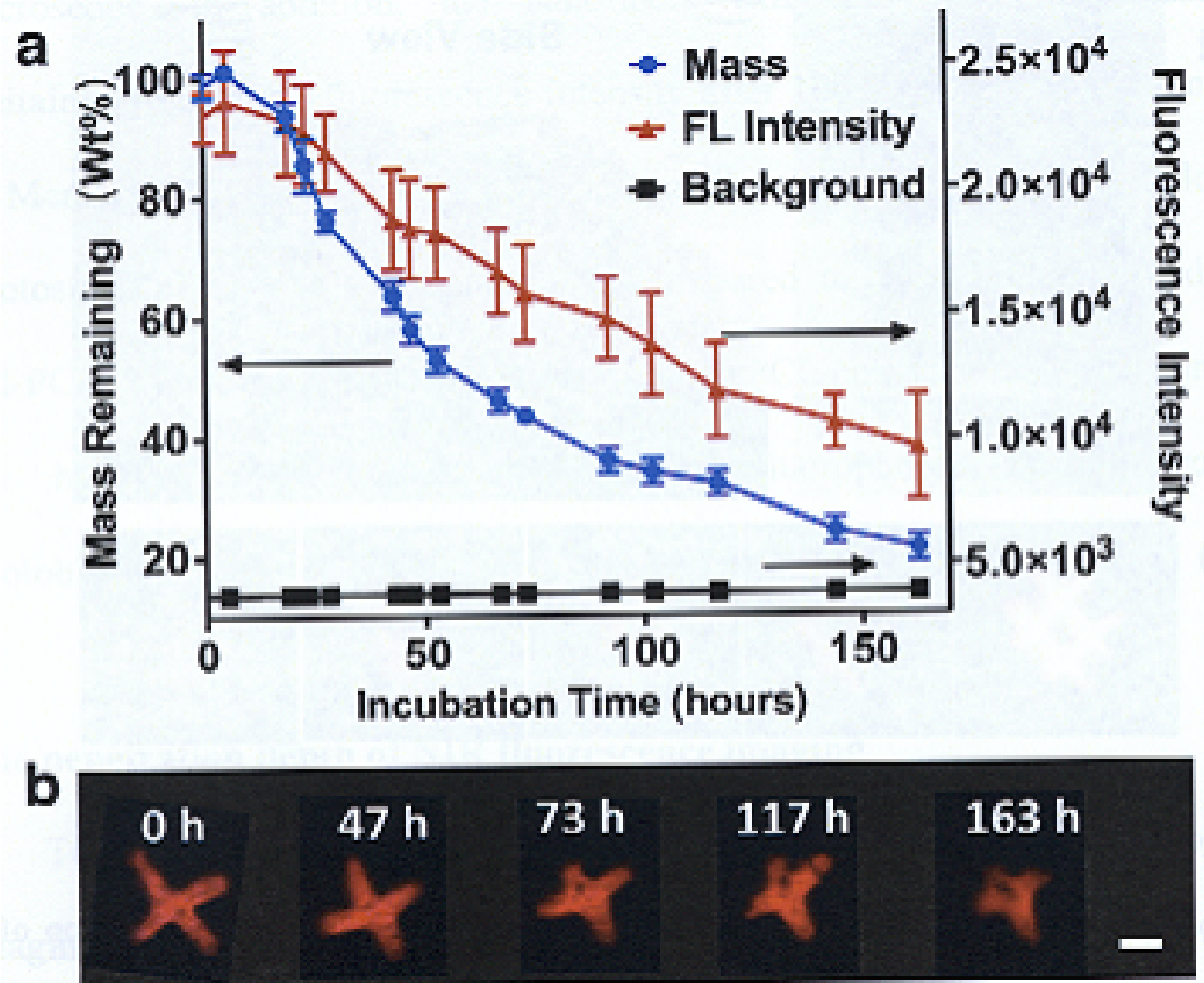

Figure 3-13. Quantification of NIR-PCL scaffold degradation in vitro in $\mathrm{NaOH}$ aqueous media. (a) Degradation measurement of NIR-PCL by fluorescence intensity and remaining mass, respectively, within 8 days. (b) NIR digital photos of a NIR-PCL scaffold after being soaked in $\mathrm{NaOH}$ aq. over different periods. Scale bar $=1 \mathrm{~cm}$.

degradation rate of the NIR-PCL depending on the concentration of $\mathrm{NaOH}$, in which the mass of the scaffold remained less than $10 \%$ after $96 \mathrm{~h}$ in $\mathrm{NaOH}(5$ M). Figure 3-13a shows that the decrease of the NIR fluorescence intensity is consistent with the mass loss of the scaffold after degradation over one week, which means the degradation result monitored by NIR fluorescence is reliable for future in vivo applications. The evolution of the NIR-PCL scaffold after being soaked in $\mathrm{NaOH} a q$. over different periods could be monitored clearly by 
the NIR fluorescence images (Figure 3-13b) obtaincd by a shortwave infrared (SWIR) Indium Gallium Arsenide (InGaAs) camera. These results suggest the promising potential of this NIR-PCL in monitoring the degradation of PCL in vivo with a non-invasive manner.

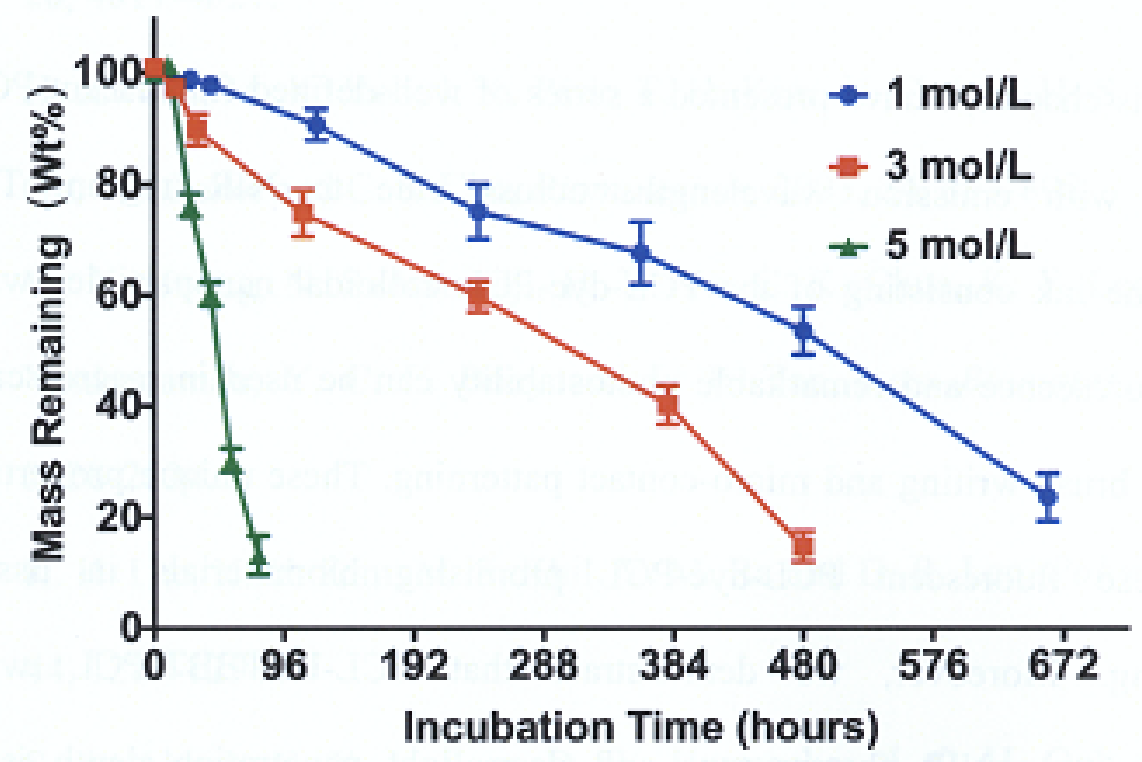

Figure 3-14. Evolution of the mass of NIR-PCL scaffolds incubated in aqueous media of $\mathrm{NaOH}$ at various concentrations at $37^{\circ} \mathrm{C}$.

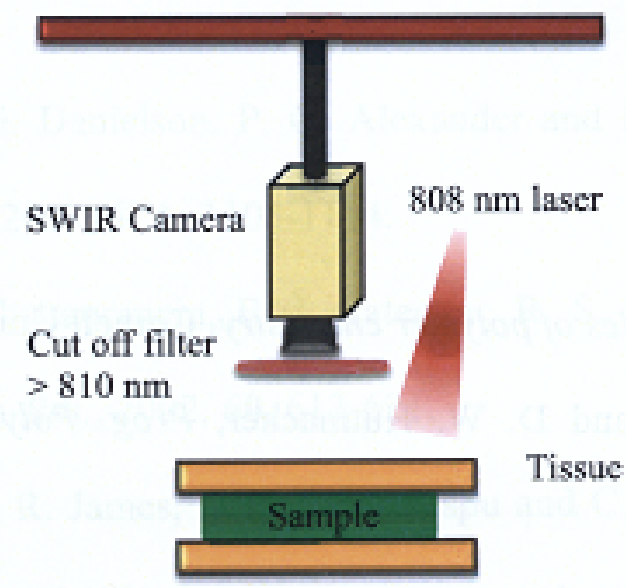


Figure 3-15. Schematic presentation of SWIR imaging system of NIR scaffold under $808 \mathrm{~nm}$ laser irradiation.

\subsection{Conclusions}

In this chapter, I have presented a series of well-defined fluorescent PCL polymers with emission wavelengths across blue to NIR region. The water-borne ink consisting of the PCL-dye-PCL colloidal nanoparticles with bright fluorescence and remarkable photostability can be used in macro-scale stamping, brush writing and micro-contact patterning. These unique properties

make these fluorescent PCL-dye-PCL promising biomaterials in tissue engineering. Moreover, we demonstrate that PCL-DPTBBT-PCL with relatively strong NIR fluorescence and deep light penetration depth is a promising biopolymer for bioimaging and monitoring the degradation of PCL scaffolds in a non-invasive way.

\subsection{References}

1. P. J. Flory, Principles of polymer chemistry, Cornell University Press 1953.

2. M. A. Woodruff and D. W. Hutmacher, Prog. Polym. Sci., 2010, 35, $1217-1256$.

3. C. Allen, Y. Yu, D. Maysinger and A. Eisenberg, Bioconjugate Chem., $1998,9,564-572$. 
4. P. Lim Soo, L. Luo, D. Maysinger and A. Eisenberg, Langmuir, 2002, 18, 9996-10004.

5. J. M. Williams, A. Adewunmi, R. M. Schek, C. L. Flanagan, P. H. Krebsbach, S. E. Feinberg, S. J. Hollister and S. Das, Biomaterials, 2005, 26, 4817-4827.

6. H. Kweon, M. K. Yoo, I. K. Park, T. H. Kim, H. C. Lee, H.-S. Lee, J.-S. Oh, T. Akaike and C. S. Cho, Biomaterials, 2003, 24, 801-808.

7. W. Y. Yeong, N. Sudarmadji, H. Y. Yu, C. K. Chua, K. F. Leong, S. S. Venkatraman, Y. C. F. Boey and L. P. Tan, Acta Biomater., 2010, 6, 2028-2034.

8. K. H. Lee, H. Y. Kim, M. S. Khil, Y. M. Ra and D. R. Lee, Polymer, 2003, 44, 1287-1294.

9. S. A. Abbah, C. X. L. Lam, D. W. Hutmacher, J. C. H. Goh and H.-K. Wong, Biomaterials, 2009, 30, 5086-5093.

10. S. H. Oh, I. K. Park, J. M. Kim and J. H. Lee, Biomaterials, 2007, 28, $1664-1671$.

11. W.-J. Li, K. G. Danielson, P. G. Alexander and R. S. Tuan, J. Biomed. Mater. Res. A, 2003, 67A, 1105-1114.

12. W.-J. Li, C. T. Laurencin, E. J. Caterson, R. S. Tuan and F. K. Ko, J. Biomed. Mater. Res., 2002, 60, 613-621.

13. S. G. Kumbar, R. James, S. P. Nukavarapu and C. T. Laurencin, Biomed. Mater., 2008, 3, 034002.

14. C. X. F. Lam, D. W. Hutmacher, J.-T. Schantz, M. A. Woodruff and S. H. 
Teoh, J. Biomed. Mater. Res. A, 2009, 90A, 906-919.

15. N. Artzi, N. Oliva, C. Puron, S. Shitreet, S. Artzi, A. bon Ramos, A. Groothuis, G. Sahagian and E. R. Edelman, Nat. Mater., 2011, 10, 704-709.

16. S. H. Kim, J. H. Lee, H. Hyun, Y. Ashitate, G. Park, K. Robichaud, E. Lunsford, S. J. Lee, G. Khang and H. S. Choi, Sci. Rep., 2013, 3, 1198.

17. N. Koike, D. Fukumura, O. Gralla, P. Au, J. S. Schechner and R. K. Jain, Nature, 2004, 428, 138-139.

18. H. J. Diao, K. Wang, H. Y. Long, M. Wang and S. Y. Chew, Adv. Healthc. Mater., 2016, 5, 529-533.

19. L. Luo, J. Tam, D. Maysinger and A. Eisenberg, Bioconjugate Chem., 2002, $13,1259-1265$.

20. R. Savic, L. Luo, A. Eisenberg and D. Maysinger, Science, 2003, 300, 615-618.

21. G. Zhang, T. L. St. Clair and C. L. Fraser, Macromolecules, 2009, 42, 3092-3097.

22. S. Huang, S. Liu, K. Wang, C. Yang, Y. Luo, Y. Zhang, B. Cao, Y. Kang and M. Wang, Nanoscale, 2015, 7, 889-895.

23. K. Wang, Y. Luo, S. Huang, H. Yang, B. Liu and M. Wang, J. Polym. Sci. A Polym. Chem., 2015, 53, 1032-1042.

24. M. Wang, S. Kumar, A. Lee, N. Felorzabihi, L. Shen, F. Zhao, P. Froimowicz, G. D. Scholes and M. A. Winnik, J. Am. Chem. Soc., 2008, 130, 9481-9491. 


\section{Chapter 4 Fluorescent amphiphilic block}

\section{copolymer for imaging probe and drug carrier}

In previous chapters, I presented a series of fluorescent PCL polymers with various emission wavelengths from visible to NIR region. These polymers are highly fluorescent in solid state and show robust photostability for long-term applications. Thus, it has large potential to use for drug carrier by fabricating amphiphilic polymers, giving fluorescence signal to track nanocarrier and drug. In this chapter, I present a fluorescent anticancer drug carrier system formed by covalently linked benzothiadiazole thiophene (BTT) in the middle of biodegradable amphiphilic polymer, poly(caprolactone)-block-poly (oligo ethylene glycol) methyl ether methacrylates (PCL-b-POEGMA) or poly(decalactone)-block-poly (oligo ethylene glycol) methyl ether methacrylates (PDL- $b$-POEGMA). The micelles formed by amiphiphilic polymers with PDL can effectively encapsulate anticancer drugs, such as doxorubicin (DOX), and quickly release DOX under acidic condition.

\subsection{Introduction}

Drug carrier plays an important role in drug delivery system that serves to improve the selectivity, treatment effectiveness and drug safety ${ }^{1,2}$. It should meet a number of stringent requirements including low toxicity, good 
biomcompatibility and biodegarability. Nowadays, many kinds of drug carrier have been explored, such as liposomes ${ }^{3-5}$ and polymer micelles ${ }^{6,7}$, to enhance the drug circulation time and increase drug effectiveness. Either liposomes or copolymer micelles system elongates the drug lifetime in blood circulation, improves the targeting efficacy and minimize the toxicity and side effects to healthy tissues. As polymer micelles, amphiphilic polymers are used since the most of drug are hydrophobic. Due to the good biodegradability, polyesters such as poly(lactic acid), (PLA), poly(lacticco-glycolic acid) (PLGA), poly(caprolactone) (PCL) are widely used in polymer drug carrier. Interestingly, natural compound $\delta$-Decalactone ${ }^{8-10}$, an FDA approved flavouring agent, has been studicd and proved that it can encapsulate higher drug content than $\mathrm{PCL}^{8}$.

Despite these advantages of existing drug carriers, it is important to track the kinetics and release of drug in biological system. Fluorescence agent is an ideal choice that can provide real-time information and high resolution. However, the physical method to incorporate fluorescent dye into drug carrier will cause leaching concern. In chapter 2 and 3 , I have presented the photostability of fluorescent polymer with tunable emission wavelength for long-term application, which was covalently linked fluorescent dye in the middle of polymer. These fluorescent polymers with high fluoreseence, robust photostability and NIR emission wavelength are suitable for tracking the release performance of drug carrier.

In this chapter, I present a fluorescent anticancer drug carrier system formed by covalently linking benzothiadiazole thiophene (BTT) in the middle 
of biodegradable amphiphilic polymer, poly(caprolactone)-block-poly(oligo ethylene glycol) methyl ether methacrylates (PCL- $b$-POEGMA) or poly(decalactone)-block-poly (oligo ethylene glycol) methyl ether methacrylates (PDL- $b$-OEGMA). The micelles formed by BTT-PDL- $b$-POEGMA (nominated as RPO-3) can effectively encapsulated anticancer drug DOX, and quickly release DOX under acidic condition. The fluorescence from polymeric carrier can indicate the location of micelles. Finally, internalization of micelles by HeLa cells and the cytotoxicity of DOX@RPO-3 micelles were studied.

\subsection{Materials and Methods}

\subsubsection{Materials and characterization}

$\varepsilon$-Caprolactone (97\%), $\delta$-Decalactone $(\geq 98 \%), \alpha$-Bromoisobutyryl bromide (BIBB) $(98 \%)$, 1,5,7-triazabicyclo[4.4.0]dec-5-ene (TBD) (98\%), Poly(ethylene glycol) methyl ether methacrylate monomer (OEGMA, $M_{\mathrm{n}} 300 \mathrm{kDa}$ ) and all solvent were purchased form Sigma Aldrich. OEGMA monomer was passed through basic alumina column before use. Dulbecco's Modified Eagle's Medium (DMEM), fetal bovine serum (FBS), penicillin /streptomycin mixture, phosphate buffered saline (PBS) and PrestoBlue cell viability reagent were purchased from Life Technologies (Singapore). Other reagents including 4', 6-diamidino-2-phenylindole (DAPI) and formalin solution were ordered from 
Sigma-Aldrich (Singapore). All reactions were carried out under $\mathrm{N}_{2}$ atmosphere unless noted specifically.

${ }^{1}$ H-NMR spectra were recorded on a Bruker AV 300 NMR spectrometer (Rheinstetten, Germany) using tetramethylsilane as an internal standard at $25{ }^{\circ} \mathrm{C}$. The number average molecular weight $\left(M_{\mathrm{w}}\right)$ and molecular weight distribution $\left(M_{\mathrm{w}} / M_{\mathrm{n}}\right)$ were measured by gel permeation chromatography (GPC) system (Agilent 1260, USA) cquipped with waters 1260 pump and a Agilent 1260 refractive index detector and a styragel HT column. Tetrahydrofuran (THF) was used as the eluent $(1 \mathrm{~mL} / \mathrm{min})$, and polystyrene was used as the standard for calibration. Transmission electron microscope (TEM) measurements were carried out on a Carl Zeiss Libra 120 Plus electron microscope operating at an acceleration voltage of $120 \mathrm{kV}$. UV-vis absorption spectra were recorded on a UV-2450 (SHIMADZU, Japan) spectrophotometer. Steady-state photoluminescence emission spectra were recorded with LS 55 (PerkinElmer, USA) fluorescence spectrometer. The particle size was measured with a dynamic light scattering (DLS) instrument, Zetasizer Nano ZS (Malvern, UK).

\subsubsection{Methods}

\section{Synthesis of polymers}

Synthesis of red fluorescent polycaprolactone (R-PCL)/polyedecalactone (R-PDL) homopolymer

The synthesis of fluorescent initiator DPTBT was described in Chapter 3. 
The monomer, $\varepsilon$-caprolactone/ $\delta$-decalactone $(5 \mathrm{mmol}, 620 \mathrm{mg} / 6 \mathrm{mmol}, 1.14 \mathrm{~g}$ ), was transferred into a flask containing the initiator stirred for 10-15 minutes to

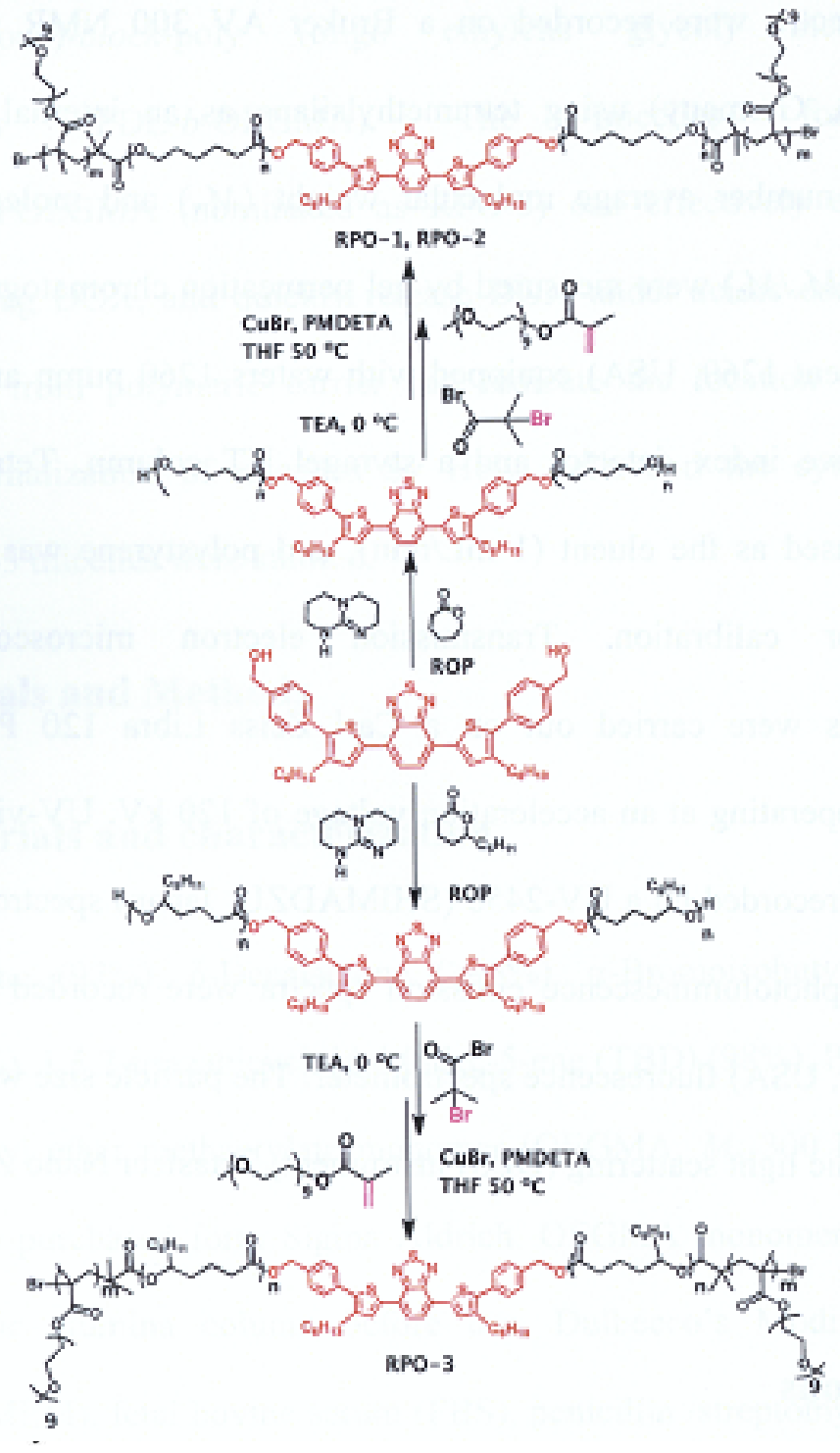

Scheme 4-1. Schematic illustration of chemical structures and synthetic route of RPO-1, RPO-2 and RPO-3 polymers.

make a homogeneous mixture. TBD was dissolved in chloroform then added 
under a nitrogen atmosphere and the mixture was allowed to react for $12 \mathrm{~h}$ at $45^{\circ} \mathrm{C}$. The obtained viscous liquid was subsequently quenched by adding acetic acid solution. The polymer was precipitated in cold methanol twice. Polymer R-PCL and R-PDL were recovered as red powder with yield of $70 \%$ viscous liquids with yield of $77 \%$ respectively.

\section{Synthesis of block copolymers RPO}

RPO copolymers were synthesized using initiator modified with BIBB. Typically, Red-PCL/PDL (0.1 mmol) was dissolved in anhydrous THF solution $(20 \mathrm{~mL})$, and then TEA $(20 \mathrm{mmol})$ was added under an argon atmosphere at 0 ${ }^{\circ} \mathrm{C}$, after $0.5 \mathrm{~h}$, the BIBB (20 mmol) was added dropwise to the mixture with magnetic stirring. The reaction was carried out at $0{ }^{\circ} \mathrm{C}$ for $2 \mathrm{~h}$ and then stirred for $24 \mathrm{~h}$ at room temperature. Then the mixture was precipitated in diethyl methanol twice and washed by water and methanol, yield is $70 \%$. Then the homopolymer R-PCL/PDL-Br (0.02 mmol), CuBr (0.04 mmol), and OEGMA $(M n=300,2.5 \mathrm{mmol})$ were dissolved in $0.6 \mathrm{mLTHF}$ in a $25 \mathrm{~mL}$ two-neck flask, and the solution was degassed with three frecze-pump-thaw cycles. Then the PMDETA $(8.0 \mathrm{uL}, 0.04 \mathrm{mmol})$ was injected into the above solution, and the mixture was degassed with two freeze-pump-thaw cycles. The polymerization was carried out at $50{ }^{\circ} \mathrm{C}$ for different desired times. The reaction mixture was diluted with THF and then passed through a short neutral $\mathrm{Al}_{2} \mathrm{O}_{3}$ column to remove the copper catalyst. The resulting solution was concentrated and poured into hexane to precipitate the product. 


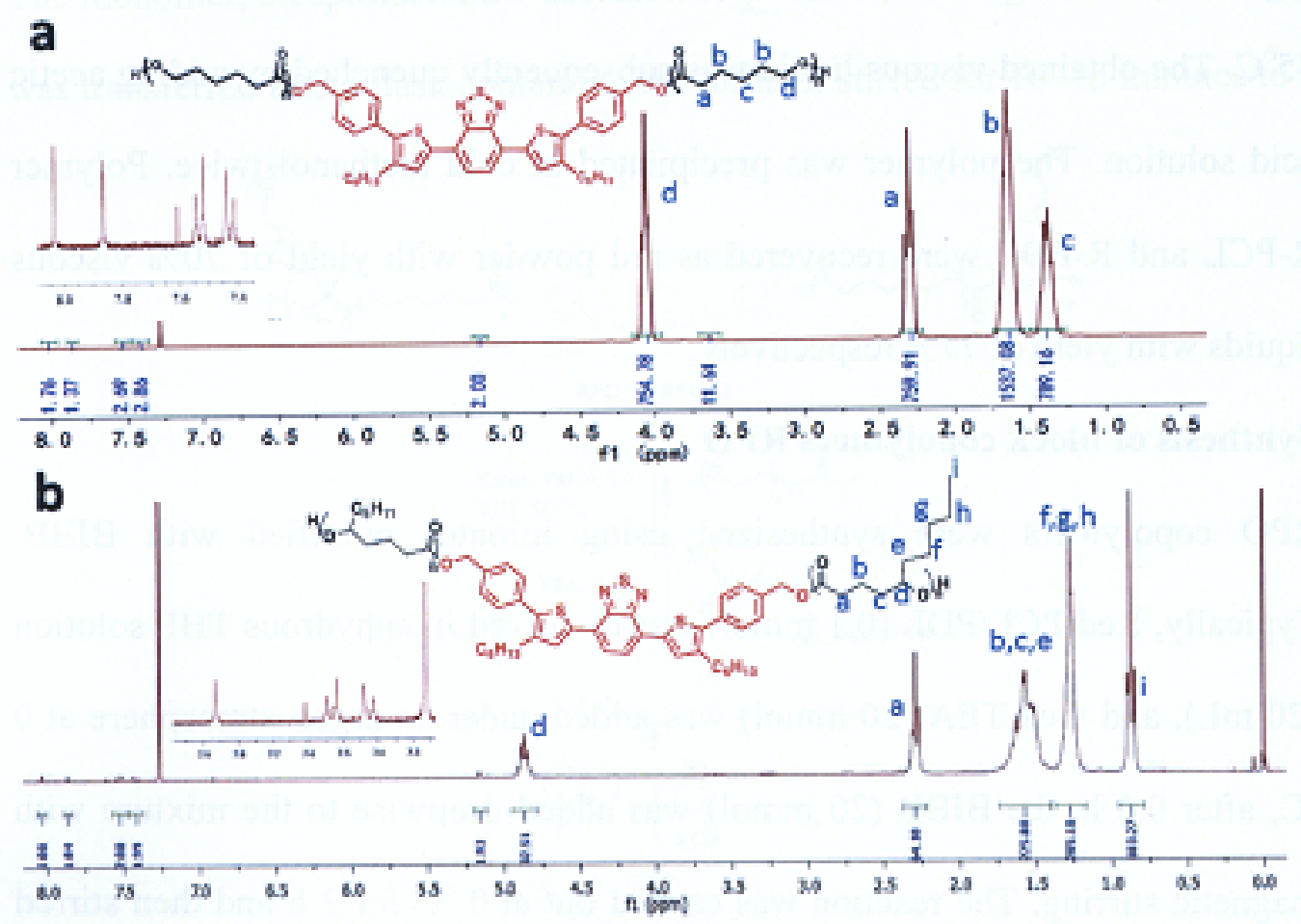

Figure 4-1. ${ }^{1} \mathrm{H}-\mathrm{NMR}\left(300 \mathrm{MHz}, \mathrm{CDCl}_{3}\right)$ spectra of homopolymer Red-PCL (a) and Red-PDL (b).

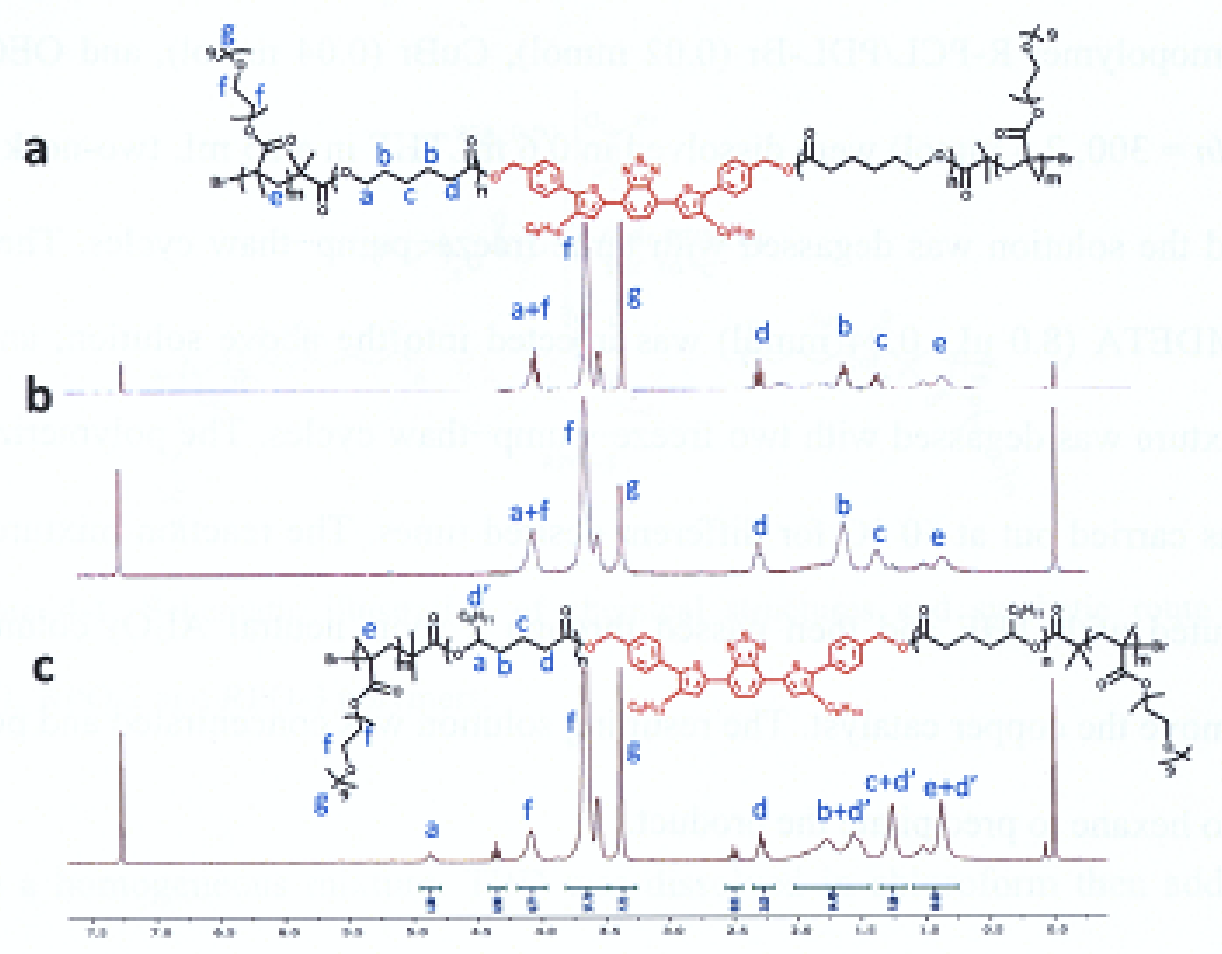


Figure 4-2. ${ }^{1} \mathrm{H}-\mathrm{NMR}\left(300 \mathrm{MHz}, \mathrm{CDCl}_{3}\right)$ spectra of amphiphilic block copolymer RPO-1 (a), RPO-2 (b) and RPO-3 (c).
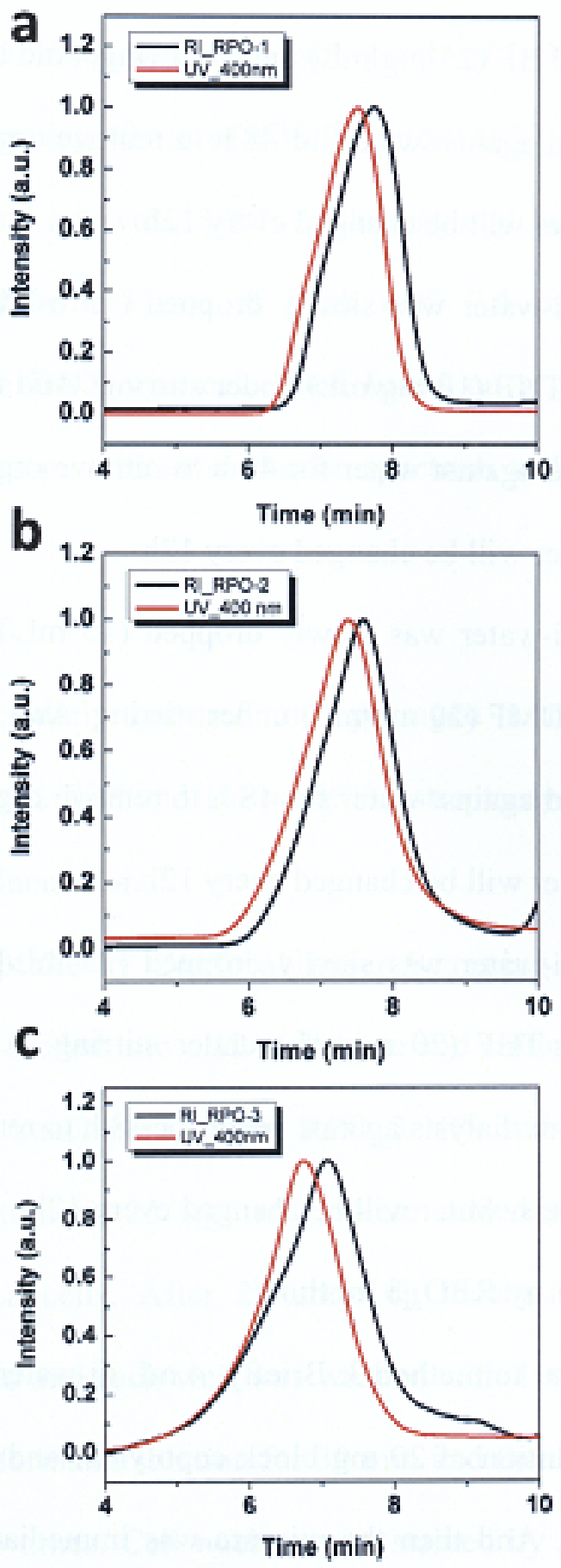
Figure 4-3. GPC results of RPO-1 (a), RPO-2 (b) and RPO-3 (c).

\section{Preparation of RPO nanoparticles}

Method 1: $4 \mathrm{~mL}$ di-water was slowly dropped $(30 \mathrm{~mL} / \mathrm{h})$ into a solution of block copolymer in THF $(25 \mathrm{mg} / \mathrm{mL})$ under stirring. And then the mixture was immediately dialyzed against water for $48 \mathrm{~h}$ to remove organic solvent. During dialysis process, water will be changed every $12 \mathrm{~h}$.

Method 2: $4 \mathrm{~mL}$ di-water was slowly dropped $(15 \mathrm{~mL} / \mathrm{h})$ into a solution of block copolymer in THF $(10 \mathrm{mg} / \mathrm{mL})$ under stirring. And then the mixture was immediately dialyzed against water for $48 \mathrm{~h}$ to remove organic solvent. During dialysis process, water will be changed every $12 \mathrm{~h}$.

Method 3: $4 \mathrm{~mL}$ di-water was slowly dropped $(15 \mathrm{~mL} / \mathrm{h})$ into a solution of block copolymer in DMF $(20 \mathrm{mg} / \mathrm{mL})$ under stirring. And then the mixture was immediately dialyzed against water for $48 \mathrm{~h}$ to remove organic solvent. During dialysis process, water will be changed every $12 \mathrm{~h}$.

Method 4: $4 \mathrm{~mL}$ di-water was slowly dropped $(15 \mathrm{~mL} / \mathrm{h})$ into a solution of block copolymer in THF $(20 \mathrm{mg} / \mathrm{mL})$ under stirring. And then the mixture stood overnight before dialysis against water for $48 \mathrm{~h}$ to remove organic solvent. During dialysis process, water will be changed every $12 \mathrm{~h}$.

\section{DOX encapsulation by RPO_3 method}

The strategy is similar to method 3. Briefly, $4 \mathrm{~mL}$ di-water was slowly dropped $(15 \mathrm{~mL} / \mathrm{h})$ into a solution of $20 \mathrm{mg}$ block copolymer and $2 \mathrm{mg}$ DOX in $1 \mathrm{~mL}$ DMF under stirring. And then the mixture was immediately dialyzed against 
water for $48 \mathrm{~h}$ to remove organic solvent. During dialysis process, water will be changed every $12 \mathrm{~h}$.

\section{In vitro DOX Release with NIR Laser Irradiation.}

In vitro release profiles of DOX were evaluated by the dialysis method. First, a dialysis bag (MWCO 3500) was filled with $1 \mathrm{~mL}$ of DOX@RPO_3 micelles soaked in a tube containing $40 \mathrm{~mL}$ of PBS 7.4 or 5.0 at $37^{\circ} \mathrm{C}$ shaking incubator. At predetermined time intervals, $3 \mathrm{~mL}$ of the external buffer was withdrawn and it was replaced with $3 \mathrm{~mL}$ of fresh PBS 7.4 or 5.0. Then release concentration of free DOX was calculated based on a calibration curve by fluorescence spectroscopy with an excitation spectrum at $495 \mathrm{~nm}$ and emission spectrum at $550 \mathrm{~nm}$.

\section{Cell Culture.}

HeLa cells were regularly cultured and passaged using DMEM medium supplemented with $10 \%$ FBS and $1 \%$ penicillin-streptomycin at $37^{\circ} \mathrm{C}$ with $5 \%$ $\mathrm{CO}_{2}$ in a humidified incubator.

In vitro Cellular Uptake by Confocal Laser Scanning Microscopy. About $10^{5} \mathrm{HeLa}$ cells were seeded into a 12 -well plate and cultured in $37^{\circ} \mathrm{C}$ with $5 \%$ $\mathrm{CO}_{2}$. After $12 \mathrm{~h}$ for attachment, empty RPO_3 NPs and DOX@RPO_3 NPs (final DOX concentration: $25 \mu \mathrm{g} \mathrm{mL} \mathrm{m}^{-1}$ ) were added to the medium and incubated with HeLa cells. After $2 \mathrm{~h}$ and $10 \mathrm{~h}$ incubation, the cell culture medium was removed and each well was washed with $1 \times$ PBS for five times. Cells were fixed by formalin solution for $30 \mathrm{~min}$ and then washed by $1 \times$ PBS extensively for three times. Cell nucleus was stained by DAPI for $10 \mathrm{~min}$ at 
room temperature and then the samples were washed three times and then added in fresh $1 \times$ PBS. Lasers of 405 and $488 \mathrm{~nm}$ were used to excite DAPI, DOX and RPO_3 respectively. The corresponding fluorescence emissions were recorded by a confocal laser scanning microscopy (LSM 710, Carl Zeiss, Germany) using a band-pass filter combination including 410-507, 495-550, and $600-700 \mathrm{~nm}$ for imaging in three individual channels (Objective: EC Plan-Neofluar 20x/0.30 M27; dimension is $1024 \times 1024)$.

\section{Cytotoxicity.}

The cytotoxicity of empty RPO_3 NPs and DOX@RPO_3 NPs in HeLa cells were studied as below. Firstly, HeLa cells were seeded into a 96 -well plate $\left(10^{4}\right.$ cells per well) and cultured at $37^{\circ} \mathrm{C}$ with $5 \% \mathrm{CO}_{2}$. After attachment over night, the culture medium was removed and replaced with fresh medium containing free DOX.HCl, empty RPO_3 NPs and DOX@RPO_3 NPs. After certain time incubation, the culture medium was removed and washed by $1 \times$ PBS for three times. Then PrestoBlue reagent diluted by DMEM medium was added to each well and incubated at $37^{\circ} \mathrm{C}$ with $5 \% \mathrm{CO}_{2}$. After $1 \mathrm{~h}$ incubation, the absorbance at $570 \mathrm{~nm}$ (reference wavelength is $600 \mathrm{~nm}$ ) was detected by Plate Reader (Tecan Infinite M200 series Pro, Tecan Asia, Singapore). All samples were tested in five replicates. Cells without treatment were used as control and corresponding cell viability was set as $100 \%$. Data were analyzed according to the protocol. 


\subsection{Results and discussion}

The synthetic route for synthesis of amphiphilic block copolymers is shown in Scheme 6-1. The ROP of homopolymer was performed at mild condition with organic catalyst 1,5,7-triazabicyclo[4.4.0]dec-5-ene (TBD). Polymcrizations were stopped by blocking the organic catalyst with acetic acid, followed by precipitation in cold methanol to remove the unconverted monomer and inactivated catalyst. Purified homopolymer molecule weight calculated by GPC indicates desired polymers with narrow distribution $(\mathrm{PDI}=1.6)$. It is clear to observe proton peaks from fluorescent dye at aromatic region (7.3-8.2 ppm). The ATRP of second hydrophilic segment was performed with copper (I) catalyst, which was removed by alumina column.

Table 4-1. Summary of polymer characterizations.

\begin{tabular}{|c|c|c|c|c|c|c|}
\hline & $\mathrm{Dp}^{1}$ & Yield & $M_{\mathrm{PCL} / \mathrm{FOL}}{ }^{1}$ & $\begin{array}{c}M_{n^{2}} \\
\mathrm{PC} / \mathrm{POL}\end{array}$ & $M n^{2}$ & $M_{w} / M_{n}$ \\
\hline RPO-1 & $\mathrm{PCL}_{129}:$ OEGMA & $71 \%$ & $74 k$ & $13 k$ & 50k & 1.9 \\
\hline RPO-2 & $\mathrm{PCL}_{91}: \mathrm{OEGMA}_{45}$ & $67 \%$ & $45 k$ & $10 k$ & 20k & 2.1 \\
\hline RPO-3 & $\mathrm{PDL}_{35}: \mathrm{OEGMA}_{30}$ & $40 \%$ & $55 k$ & $8 k$ & 23k & 3.3 \\
\hline
\end{tabular}

1. Molecular weight and repeat unit calculated by ${ }^{1} \mathrm{H}-\mathrm{NMR}$

2. Molecular weight was calculated by GPC with THF as eluent.

GPC using THF as the eluent phase gave a size distribution with relatively narrow polydispersity for all homopolymers and block copolymers. Meanwhile, 
the UV trace of absorption from fluorescent dye matched RI trace very well (Figure 4-3 a-c), which suggested the fluorescent dye was synthesized to polymers. However, the $M_{\mathrm{n}}$ observed by GPC is lower than molecular weight calculated by NMR.

I consider taking RPO-1 polymer as a model to form nanoparticles by nanoprecipitation, which has previously been employed successfully for preparation of micelles. THF and DMF were chosen as good solvent. In particular, DMF is co-solvent for polymers and DOX. As shown in Figure 4-4 a, RPO-1 nanoparticles prepared by Method 1 indicated a mixture of spherical and crystallized rod-like micelles. When the concentration and injection rate of water decreased, it showed smooth and spherical nanoparticles (Figure 4-4 b), with a narrow size distribution measured by DLS. However, it shows rough surface of spherical nanoparticles that mixed with smaller micelles (Figure 4-4 c), when DMF was chosen as good solvent. Furthermore, the TEM showed short worm-like structure (Figure 4-4 d) when the RPO-1 mixture in DMF quenched by water stood overnight before dialysis. In addition, RPO-2 with lower $M_{\mathrm{n}}$ and RPO-3 with amorphous PDL showed spherical micelles and mixture of worm-like (Figure 4-4 e) and smaller micelles (Figure 4-4 f), respectively. The difference of morphology was contributed to the highly crystalline ability of PCL chain. 

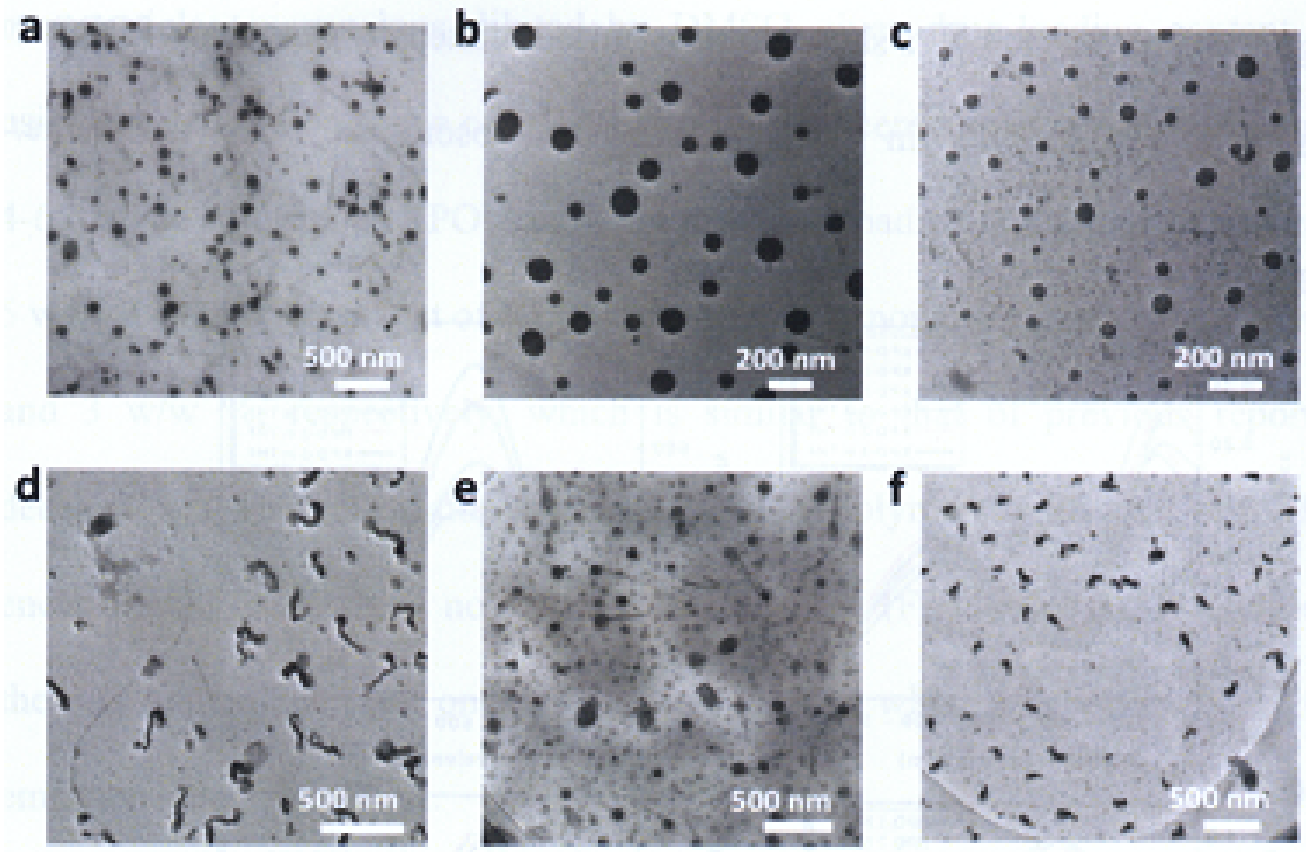

Figure 4-4. Representative TEM results of RPO-1 prepared by Method 1 (a), RPO-1 prepared by Method 2 (b), RPO-1 prepared by Method 3 (c), RPO-1 prepared by Method 4 (d), RPO-2 prepared by Method 4 (e) and RPO-3 prepared by Method 4 (f).

It is interesting to investigate the optical propertics change caused by different crystalline PCL and amorphous PDL chain. There is no significant difference among three polymers in THF and their nanoparticles suspension in water from absorption spectra (Figure $4-5 \mathrm{~d}$ ), which means no obvious intra-molecular interaction of organic dye. However, in emission spectra (Figure 4-5 e), the fluorescence pcaks of RPO-2 and RPO-3 nanoparticles showed a slightly redshift compared to that of polymers in THF. The rigidity structure caused by highly crystallized PCL provided fluorescence peaks, which is the same as that of polymers in THF. It exhibits similar emission intensity in organic solvent. However, as shown in non-normalized emission spectra 
(Figure 4-5 b), RPO_3 NPs shows higher emission intensity than that of RPO-2 NPs and RPO-1 NPs in water, when the absorbance was the same.

a

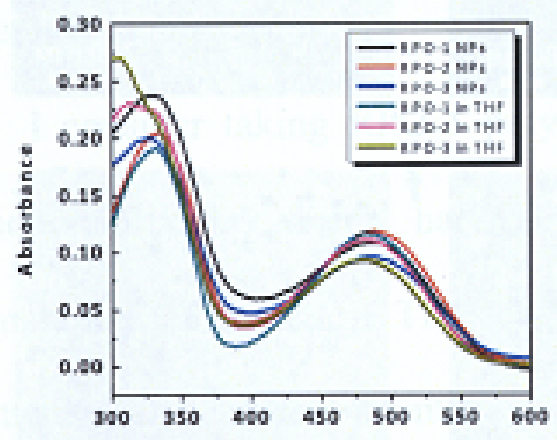

C

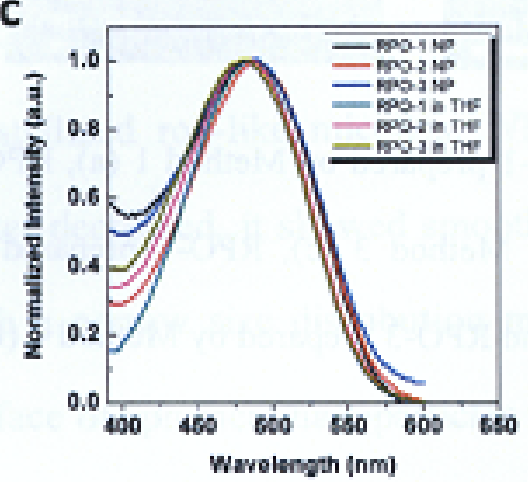

b

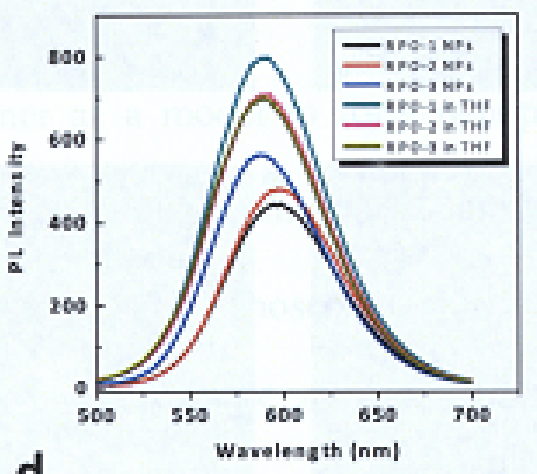

d

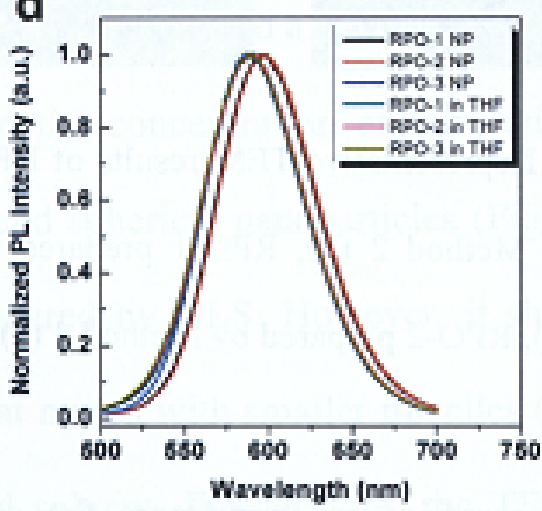

Figure 4-5. UV-vis absorption spectra (a) and emission spectra (b) of three polymers at same concentration in THF and water. Normalized UV-vis absorption spectra (c) and emission spectra (d).

The block copolymers that act as drug carriers was investigated by using DOX as a drug model, since the emission spectrum of DOX is distinguishable to that of block copolymers in organic solvent (Figure 4-6 c). The amount of DOX encapsulated in each micelle formulation was calculated using fluorescence spectrometer. The fluorescence intensity at $550 \mathrm{~nm}$ of 
nanoparticles suspensions diluted by DMSO gives drug-loading content by using the calibration curve of DOX of various concentrations in DMSO (Figure 4-6 d). The amorphous RPO-3 polymer provided loading content of DOX up to $5 \mathrm{w} / \mathrm{w} \%$ compared to that of RPO- 1 and RPO-2 nanoparticles lower to $4 \mathrm{w} / \mathrm{w} \%$ and $3 \mathrm{w} / \mathrm{w} \%$, respectively, which is similar to that of previous reported decalactone. The absorption spectra of RPO polymers with PCL or PDL cncapsulated DOX show no significant difference (Figure 4-6 a). In addition, there is a shoulder peak on the emission spectra, which is contributed to the emission peak from DOX.

Figure 4-6e shows the in vitro drug release profiles of DOX@RPO micelles at various $\mathrm{pH}$ values $(\mathrm{pH}=5.0$ and $\mathrm{pH}=7.4)$. The micelle with $\mathrm{PCL}$ chain as hydrophobic segment, RPO-1 and RPO-2, showed no significant increase at $\mathrm{pH}=5.0$ than that of $\mathrm{pH}=7.4$. However, the $\mathrm{RPO}-3$ with PDL as hydrophobic segment exhibits up to $60 \%$ release ratio at $\mathrm{pH}=5.0$, which is dramaticly higher than that of $\mathrm{RPO}-3$ at $\mathrm{pH}=7.4$. The release results indicate that the shorter PCL chain of RPO-2 is $\mathrm{pH}$ sensitive as compared to longer PCL chian RPO-1. 

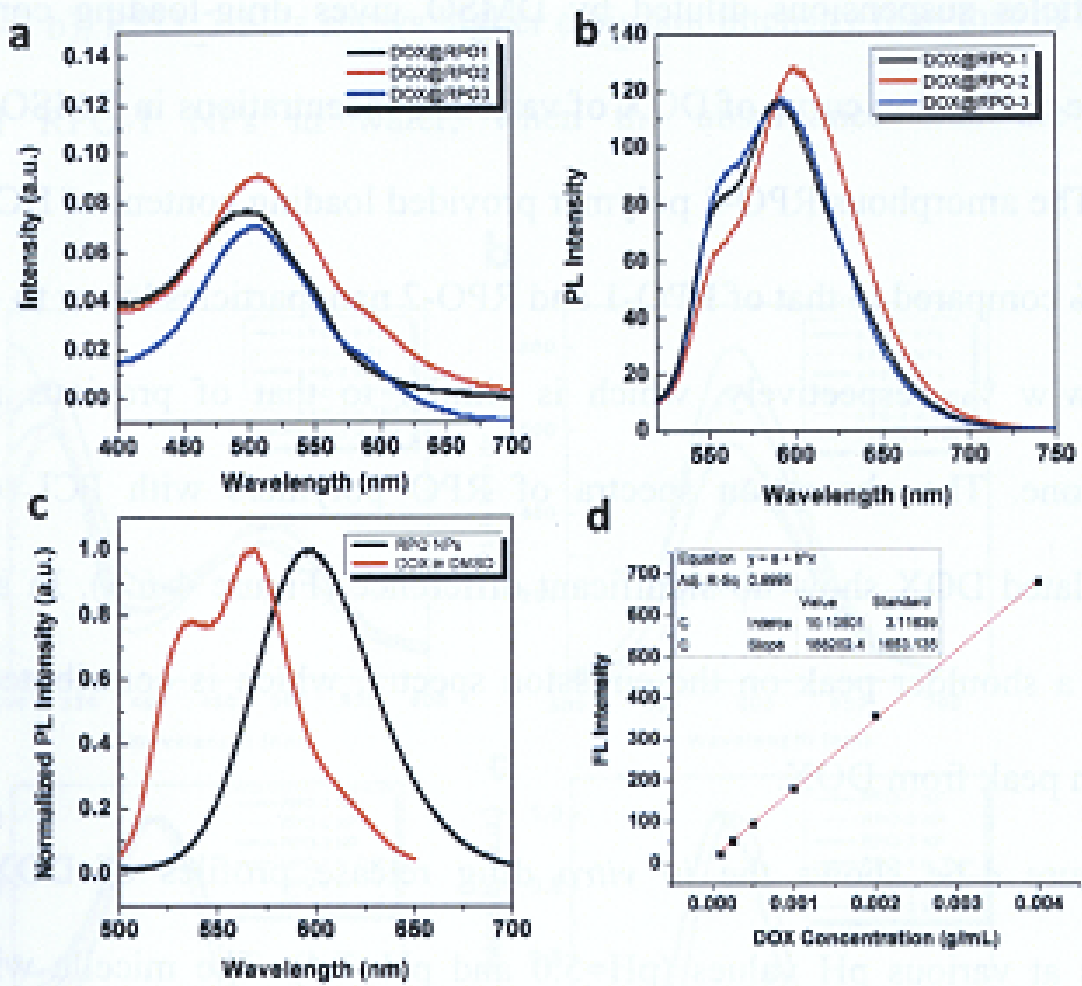

d

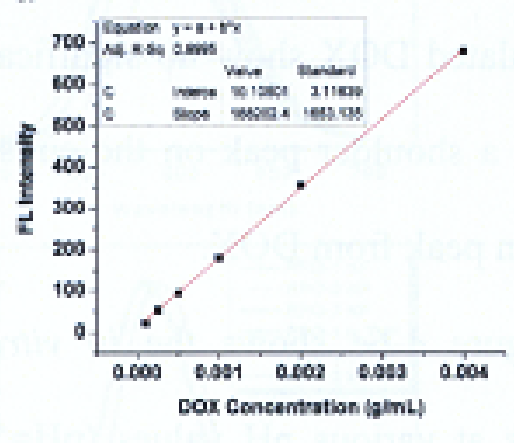

e

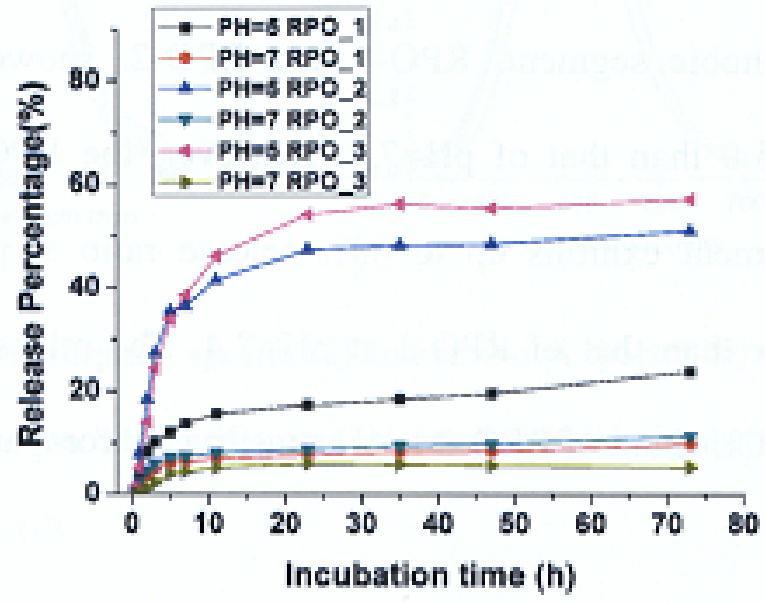

Figure 4-6. UV-vis absorption (a) and emission spectra (b) of RPO polymers encapsulated DOX. Emission spectra of DOX in DMSO and RPO NPs in water excited at $488 \mathrm{~nm}$ (c). Calibration curve of DOX concentration $v s$ fluorescence intensity at 550 nm (d). Release profile of DOX@RPO within 3 days in $\mathrm{pH}=5.0$ or 7.4 aqueous solution at $37{ }^{\circ} \mathrm{C}$ incubation $\left(\mathrm{CDOX}=50 \mu \mathrm{g} \mathrm{mL} L^{-1}\right)(\mathrm{e})$. 


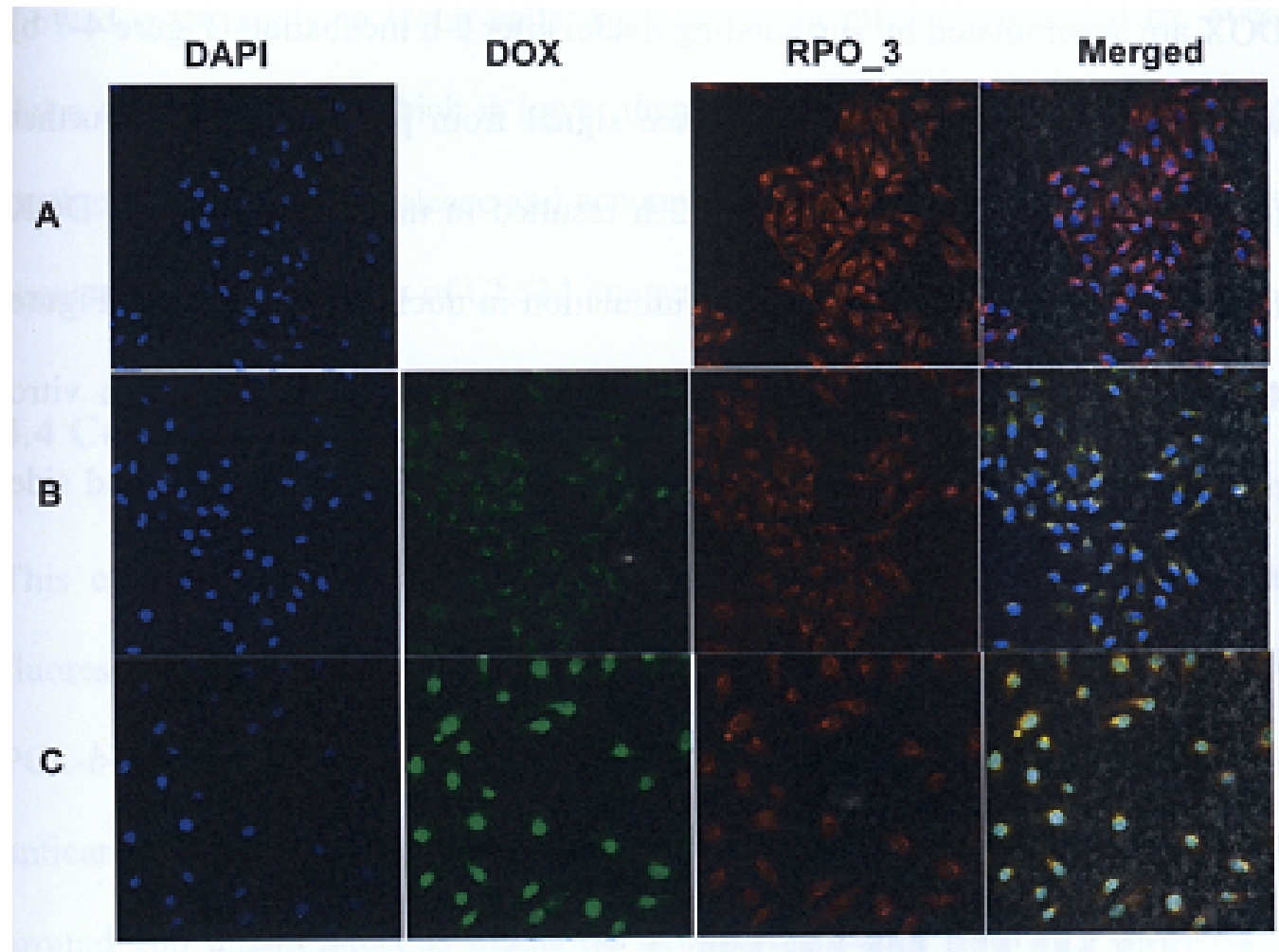

Figure 4-7. DOX internalization and localization in HeLa cells after incubation with empty RPO_3 NPs (A), DOX@RPO_3 NPs after $2 \mathrm{~h}$ incubation (B) and DOX@RPO_3 NPs after 10h incubation, respectively. The fluorescence of DAPI $\left(\lambda_{\mathrm{cx}}=405 \mathrm{~nm}\right)$, DOX $\left(\lambda_{\mathrm{ex}}=488 \mathrm{~nm}, \lambda_{\mathrm{cm}}=495-550 \mathrm{~nm}\right)$, and RPO_3 $\left(\lambda_{\mathrm{cx}}=488 \mathrm{~nm}\right.$, $\lambda_{\mathrm{cm}}=600-700 \mathrm{~nm}$ ) was pseudo labeled with blue, green, and red, respectively.

The cellular uptake behavior was further evaluated by confocal laser scanning microscopy (CLSM). The empty RPO-3 NPs can be internalized into cytoplasm after $2 \mathrm{~h}$ incubation (Figure 4-7 a). Due to the slight overlap of emission peak of DOX and RPO-3 NPs under $488 \mathrm{~nm}$ excitation, the bandpass filter was used to separate the their emission signal, which were $495-550 \mathrm{~nm}$ for DOX and $600-700 \mathrm{~nm}$ for RPO-3 NPs, respectively. It is clearly observed that 
DOX are accumulated by surrounding nuclei after $2 \mathrm{~h}$ incubation (Figure $4-7 \mathrm{~b}$ ), which is overlapped with fluorescence signal from polymer RPO-3. Further increase of the incubation time to $12 \mathrm{~h}$ resulted in more release of the DOX from the micelles and significant accumulation in nuclei of HeLa cells (Figure 4-7 c). The sustainable release behavior, which is similar to that of in vitro release profile, is important to minimize the undesired drug leaching and side effects of toxicity.
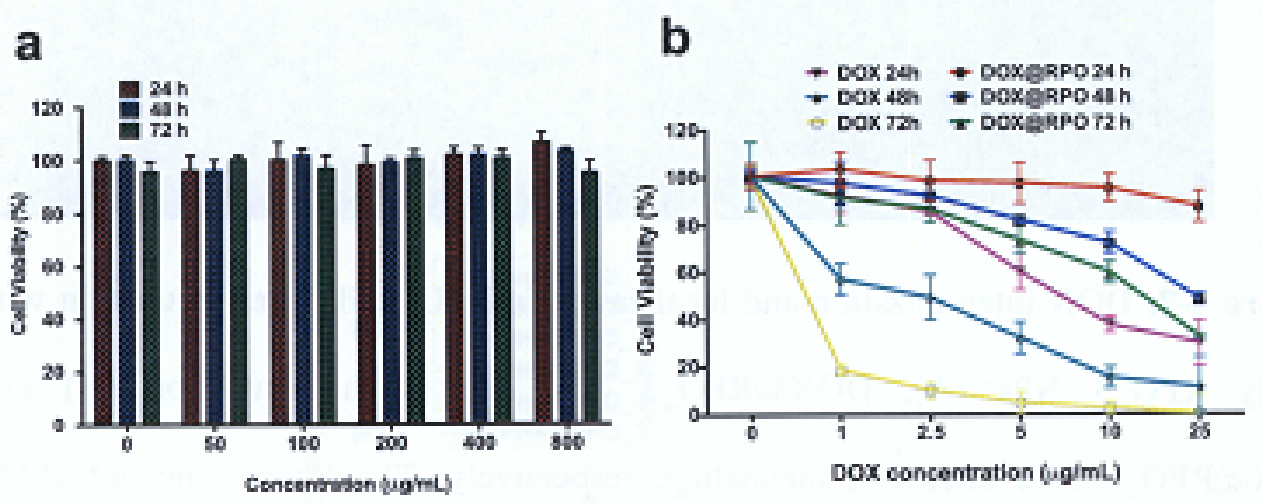

Figure 4-8. Cytotoxicity of HeLa cells tested by PrestoBlue assay after treatments with various concentrations of RPO_3 micelles for 24,48 , and $72 \mathrm{~h}$, respectively (a). Cell viability of HeLa cells tested by PrestoBlue assay after treatments with various DOX concentrations of DOX@RPO_3 NPs and frec DOX for 24,48, and 72 h, respectively (b). Data were shown as means $\pm \operatorname{SD}(n=5)$.

Finally, we investigated the cytotoxicity of blank RPO-3 micelles and DOX@RPO-3 micelles vs carrier-free DOX $\cdot \mathrm{HCl}$. Figure 4-8 a shows that no obvious toxicity was observed in HeLa cells, which were incubated with a RPO-3 concentration up to $800 \mu \mathrm{g} \mathrm{mL}{ }^{-1}$. However, the DOX@RPO-3 micelles 
showed cytotoxicity to HcLa cells, such as $25 \mu \mathrm{g} \mathrm{mL}^{-1}$ DOX killed ca. $60 \%$ HeLa cells over $72 \mathrm{~h}$, which is lower than that of free DOX $\cdot \mathrm{HCl}$. It is mainly attributed to the slower release and accumulation of DOX in micelles, which is consistent with the results of CLSM images.

\subsection{Conclusions}

This chapter presented a novel anticancer drug delivery system formed by fluorescent and biodegradable amphiphilic polymers, which consists of PCL- $b$-OEGMA covalently bonded with fluorescent dye in the middle. The anticancer drug DOX was encapsulated into RPO micelles with diameter around $100 \mathrm{~nm}$ in aqueous media by nanoprecipitation. Both in vitro release data and confocal fluorescence images showed enhanced release of DOX at $\mathrm{pH}$ 5. The released DOX further accumulated into nuclei after increase of incubation time. Moreover, the dynamic release of drug was observed by distinguishable fluorescence of DOX and fluorescent nanocarrier. In addition, no obvious death of HeLa cells was observed in non-toxic blank polymer micelles, and significant death was observed due to the treatment of DOX. These results suggested that the anticancer drug in fluorescence tracking carrier is a promising drug delivery system for future animal study and clinic trials.

\subsection{References}

1. J. W. Yoo, D. J. Irvine, D. E. Discher and S. Mitragotri, Nat. Rev. Drug Discov., 2011, 10, 521-535. 
2. G. Tiwari, R. Tiwari, B. Sriwastawa, L. Bhati, S. Pandey, P. Pandey and S. Bannerjee, Int. J. Pharm. Investig., 2012, 2, 2-11.

3. L. Sercombe, T. Veerati, F. Moheimani, S. Y. Wu, A. K. Sood and S. Hua, Front Pharmacol., 2015, 6.

4. T. M. Allen and P. R. Cullis, Adv. Drug Deliv. Rev. , 2013, 65, 36-48.

5. B. S. Pattni, V. V. Chupin and V. P. Torchilin, Chem. Rev., 2015, 115, 10938-10966.

6. P. Chandna, J. J. Khandare, E. Ber, L. Rodriguez-Rodriguez and T. Minko, Pharmaceut. Res., 2010, 27, 2296-2306.

7. T. Jia, S. Huang, C. Yang and M. Wang, Mol. Pharm., 2016.

8. K. K. Bansal, D. Kakde, L. Purdie, D. J. Irvine, S. M. Howdle, G. Mantovani and C. Alexander, Polym. Chem., 2015, 6, 7196-7210.

9. D. Kakde, V. Taresco, K. K. Bansal, E. P. Magennis, S. M. Howdle, G. Mantovani, D. J. Irvine and C. Alexander, J. Mater. Chem. B, 2016, 4, 7119-7129.

10. M. T. Martello, A. Burns and M. Hillmyer, ACS Macro Lett., 2012, 1, 131-135. 


\section{Chapter 5 A narrow-bandgap benzobisthiadiazole derivative with high near-infrared photothermal}

conversion efficiency and robust photostability

\section{for cancer therapy}

In previous chapters, I presented a series of fluorescent polymers with highly fluorescence and good photostability using for imaging agent of tissue engineering and drug carricr. In particular, the emission wavelegnth of fluorescent polymer was extented to NIR region, which is suitable for biological application. Once the emission wavelength extends to NIR region, the fluorescence intensity will decrease dramaticlly, while the energy absorbed is converted to heat energy. This chapter focuses on a new photothermal agent based on colloidal nanoparticles formed by a small-molecular dye, benzo $\left[1,2-c ; 4,5-c^{\prime}\right]$ bis $[1,2,5]$ thiadiazole-4,7-bis (5-(2-ethylhexyl)thiophene. These nanoparticles showed strong near-infrared absorption, robust photostability and high therapeutic efficiency for photothermal treatment of cancer cells. This work has been published in Chemistry Communication in 2015. 


\subsection{Introduction}

As one of major causes of death, cancer and malignant tumors have greatly threatened human health. Photothermal therapy (PTT) that converts absorbed light energy into heat has attracted great attention due to many advantages, such as high specificity, minimal invasiveness, low toxicity to normal tissues, and excellent anti-cancer efficacy. ${ }^{1-3}$ For the in vivo applications of PTT, therapeutic agents with strong optical absorbance in the near-infrared (NIR) therapeutic optical window $(700-1000 \mathrm{~nm})$ are preferable to minimize light attenuation in tissues in order to achieve a maximal light penetration depth. Moreover, biological chromophores will be affected minimally in this region. ${ }^{4}$ The localization of laser energy for determining the heating profile and subsequent PTT effect can also be simulated beforehand. ${ }^{5,6}$ In addition, the agents used for PTT should be biocompatible and non-toxic. In recent years, a variety of NIR-absorbing inorganic nanomaterials, such as gold nanostructures, ${ }^{7-12}$ Pd nanosheets, ${ }^{13}$ carbon nanomaterials, ${ }^{14}, 15$ rare earth ions doped nanocrystals, ${ }^{16}$ copper sulfide nanoparticles, ${ }^{17,}{ }^{18}$ tungsten oxide nanowires, ${ }^{19}$ quantum $\operatorname{dots}^{20}$ and porous silicon ${ }^{21}$ have been explored for PTT. Although the high efficacy for PTT treatment of cancers has been demonstrated using most of these inorganic nanomaterials, they are non-biodegradable and tend to retain in the body for a long period of time, raising the issue of long-term toxicity in potential clinical applications. ${ }^{22}$ 
As a consequence, photothermal agents based on organic NIR-absorbing materials have attracted increasing attention recently. For instance, NIR-absorbing agents based on conjugated polymers with appropriate aromatic structures, such as polyaniline, poly-(3,4-ethylenedioxythioiphene):poly(4-styrenesulfonate)(PEDOT:PS S), polypyrrole, and dopamine-melanin exhibit high photothermal conversion efficacy in PTT-based cancer treatment. ${ }^{23-26}$ Despite the encouraging PTT therapeutic effects obtained from those polymeric nanoparticles, the biodegradation behavior of conjugated polymers remains unclear.

On the other hand, small molecular organic dyes such as indocyanine green $(\mathrm{ICG})^{27,28}$ and methylene blue ${ }^{29}$ have been used not only in fluorescence-based imaging, but also as PTT agents. These small molecular PTT agents show advantages such as their well-defined molecular structures, good synthetic reproducibility in contrast to the batch-to-batch difference in polymer synthesis, and the potentially improved biodegradability. The further exploration of these small molecular PTT agents, nevertheless, is limited by their poor photostability and short circulation time. ${ }^{30}$ Recently, a synthetic heptamethine indocyanine dye encapsulated by PEG-grafted poly(maleicanhydride-alt-1-octadecene) showed improved photostability than ICG and has been used as a photothermal agent for in vivo cancer therapy. ${ }^{27}$ Other nanoparticles (NPs), for example, formed by 
self-assembly of porphyrin-lipid conjugates have been recently reported as a new type of PTT agent. ${ }^{31}$

In this chapter, I present a new photothermal agent based on a small-molecular dye, benzo[1,2-c;4,5-c']bis[1,2,5]thiadiazole-4,7-bis (5-(2-ethylhexyl)thiophene (BBTEHT). BBT unit, due to its hypervalent sulfur atoms stabilized in quinoidal structures in a conjugated backbone, is a strong electron accepting unit that ensures strong NIR absorption of BBTEHT in the therapeutic optical window. ${ }^{32-38}$ The NPs formed by BBTEHT molecules were prepared through nanoprecipitation in the presence of Pluronic ${ }^{\circledR} 127$ as the stabilizer, which is commercially available and has been widely used to encapsulate hydrophobic therapeutic agents for drug delivery. ${ }^{39,}{ }^{40}$ Under 808-nm NIR laser irradiation, BBTEHT NPs showed improved photostability and higher photothermal conversion efficacy compared to that of commercial gold $(\mathrm{Au})$ nanorods. In addition, BBTEHT NPs at a concentration as low as 35 $\mu \mathrm{g} / \mathrm{mL}$ achieved an excellent therapeutic efficacy with nearly $100 \%$ cancer cell death.

\subsection{Materials and methods}

\subsubsection{Materials and agents}

All chemicals and were purchased from Sigma-Aldrich. All solvents were used as received. Anhydrous solvents were obtained through a solvent purification system (Innovative Technologies). ${ }^{1} \mathrm{H}$ NMR spectra were recorded on a Bruker 
AV 300 NMR spectrometer (Rheinstetten, Germany) using tetramethylsilane as an internal standard at $25{ }^{\circ} \mathrm{C}$. Transmission electron microscope (TEM) measurements were carried out on a Carl Zeiss Libra 120 electron microscope operating at an acceleration voltage of $120 \mathrm{kV}$. UV-vis absorption spectra were recorded on a UV-2450 (SHIMADZU, Japan) spectrophotometer. Steady-state photoluminescence emission spectra were recorded with LS 55 (PerkinElmer, USA) fluorescence spectrometer. Fluorescent quantum yield was measured by Edinburgh fluorescence spectrometer FLS980. The particle size was measured with a dynamic light scattering (DLS) instrument, Zetasizer Nano ZS (Malvern, UK).

\subsubsection{Synthesis of BBTEHT}

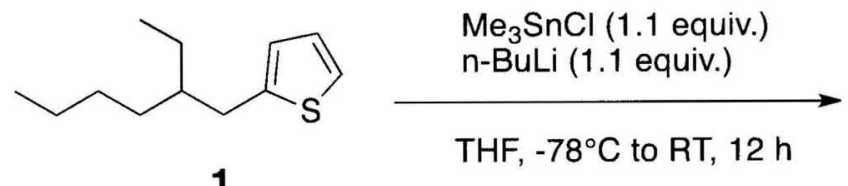

1

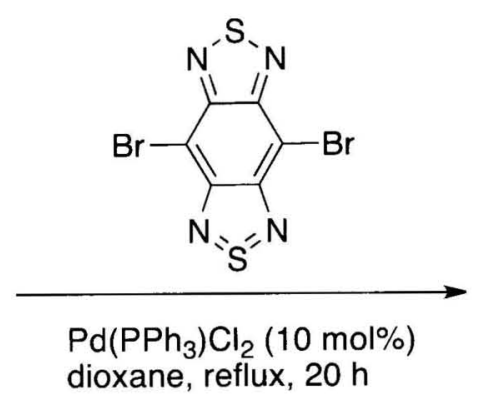

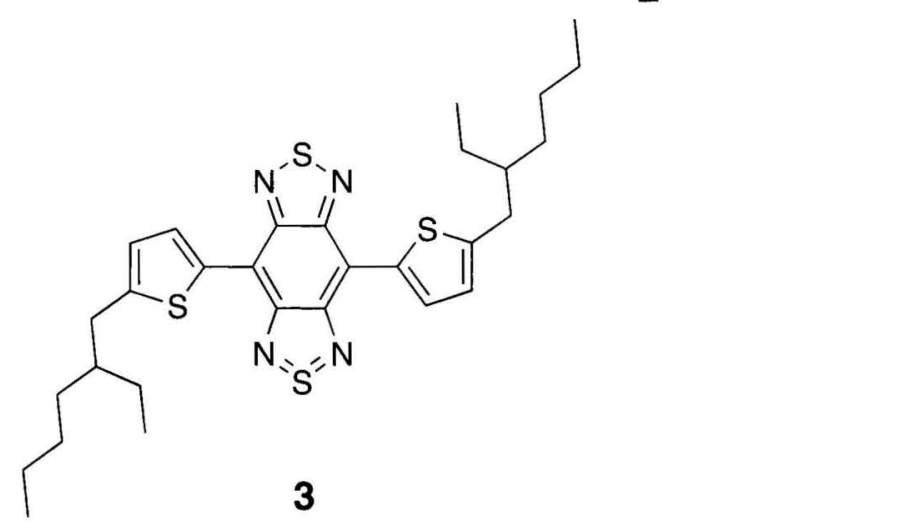

Scheme 5-1. Synthetic route of BBTEHT compound

(5-(2-ethylhexyl)thiophen-2-yl)trimethylstannane(compound 2). In a two neck 
$100 \mathrm{~mL}$ round bottom flask, to the solution of 2-ethylhexyl)thiophen (1) (2.11 g, $10.75 \mathrm{mmol})$ in $50 \mathrm{~mL}$ of THF was dropwise added $n$-BuLi $(11.8 \mathrm{mmol}, 4.72$ $\mathrm{mL}$ of $2.5 \mathrm{M}$ in hexane) at $-78{ }^{\circ} \mathrm{C}$ under $\mathrm{Ar}$ atmosphere. The resulting mixture was stirred for $1 \mathrm{~h}$ at the same temperature and then allowed to warm to $0{ }^{\circ} \mathrm{C}$ and stirred at this temperature for $30 \mathrm{~min}$, and again cooled to $-78{ }^{\circ} \mathrm{C}$, followed by slow addition of $\mathrm{Me}_{3} \mathrm{SnCl}(11.8 \mathrm{mmol}, 11.8 \mathrm{~mL}$ of $1.0 \mathrm{M}$ in hexane). The resulting solution was slowly warmed to room temperature and stirred overnight. The reaction was quenched with water and extracted with dichloromethane $(30 \mathrm{~mL} \times 2)$, the combined organic layers was dried over $\mathrm{MgSO}_{4}$, filtered, concentrated to give a yellow oil (3.55 g, 92\%) compound (2) which was used directly for the next step without further purification.

\section{4,8-di(2-ethylhexyl)thiophen-2-yl)-benzo[1,2-c;4,5-c']bis[1,2,5]thiadiazole}

(compound 3). 4,8-dibromo--benzo[1,2-c;4,5-c']bis[1,2,5]thiadiazole (1.03 g, $2.9 \mathrm{mmol}), \mathrm{Pd}\left[\mathrm{PPh}_{3}\right]_{2} \mathrm{Cl}_{2}(0.21 \mathrm{~g}, 0.29 \mathrm{mmol})$, Compound $2(2.48 \mathrm{~g}, 6.9 \mathrm{mmol})$ and anhydrous 1,4-dioxane $(43 \mathrm{~mL})$ was added into a round bottom $100 \mathrm{~mL}$ Schlenck flask. The mixture was bubbled with Ar for $10 \mathrm{~min}$ and then refluxed under $\mathrm{N}_{2}$ overnight. The mixture was cooled down to room temperature under $\mathrm{N}_{2}$. Then 2-trimethylstanny-3-hexylthiophene $(1.2 \mathrm{~mL})$ and $\mathrm{Pd}\left[\mathrm{PPh}_{3}\right]_{2} \mathrm{Cl}_{2}(0.2 \mathrm{~g})$ was added to the reaction mixture. The mixture was bubbled with Ar for $10 \mathrm{~min}$ and refluxed again under $\mathrm{N}_{2}$ for $24 \mathrm{~h}$. The mixture was cooled down to room temperature and poured into methanol $(150 \mathrm{~mL})$. The crude product was purified further by column chromatography (silica gel, hexane/toluene (4:1, 
$\mathrm{v} / \mathrm{v})$ ) to give dark green solid BBTEHT (337mg, 20\%). ' $\mathrm{H}$ NMR (400 MHz, $\left.\mathrm{CDCl}_{3}\right): \delta=8.71(\mathrm{~s}, 2 \mathrm{H}), 6.90(\mathrm{~s}, 2 \mathrm{H}), 2.82(\mathrm{~d}, J=6.9 \mathrm{~Hz}, 4 \mathrm{H}), 1.77(\mathrm{~s}, 2 \mathrm{H})$, $1.30(\mathrm{br}, \mathrm{m}, 16 \mathrm{H}), 0.87(\mathrm{~m}, 12 \mathrm{H}) \mathrm{ppm} ;{ }^{13} \mathrm{C}$ NMR (100 MHz, $\left.\mathrm{CDCl}_{3}\right): \delta=$ $150.97,150.79,135.54,132.25,126.51,113.18,34.98,34.36,32.41,28.84$, 25.73, 23.13, 14.22, 10.94 ppm; MALDI-TOF-MS m/z: $582.3\left(\mathrm{M}^{+}\right) ; \mathrm{M}_{\mathrm{w}}$ calcd. for $\mathrm{C}_{30} \mathrm{H}_{38} \mathrm{~N}_{4} \mathrm{~S}_{4}=582.9 ; \mathrm{T}_{\mathrm{m}}=122 ; \mathrm{FT}-\mathrm{IR}\left(\mathrm{KBr}, \mathrm{cm}^{-1}\right): 2956,2923,2857,1442$, $1376,1115,932,869$.

\section{General procedure for the synthesis of the nanoparticles:}

A mixture of $5 \mathrm{mg}$ of BBTEHT and $83 \mathrm{mg}$ of Pluronic 127 was completely dissolved in $1 \mathrm{~mL}$ of THF overnight. Then $10 \mathrm{~mL}$ of deionized-water was quickly injected into the mixture under vigorous stirring at room temperature. After being stirred for $5 \mathrm{~min}$, the dispersion was dialyzed against deionized-water by $4 \mathrm{KDa}$ dialysis membranes for $48 \mathrm{~h}$ to remove THF. The NPs were separated by centrifugation at $10000 \mathrm{rpm}$ for $5 \mathrm{~min}$ to remove unencapsulated surfactant and then redispersed in deionized-water before characterization and cell study. The concentrations used for following measurements and cell study are calculated based on the amount of BBTEHT used in NP preparation.

\subsubsection{General materials characterization:}

Scanning electron microscopy (SEM) was performed on a Field Emission Scanning Electron Microscopy (FESEM, Model JEOL JSM 6701F) operated at 
$10 \mathrm{kV}$. Transmission electron microscope (TEM) measurements were carried out on a TEM Carl Zeiss Libra 120 Plus operating at an acceleration voltage of $120 \mathrm{kV}$. UV-vis transmission spectrum was recorded on a Varian Cary 4000 UV-Vis spectrophotometer. ${ }^{1} \mathrm{H}$ NMR spectra and ${ }^{13} \mathrm{C}$ NMR spectra were recorded on a Varian Inova-400 Instrument (400 MHz). MADLI-TOF MS spectra were carried on MALDI TOF/TOF ABI 4800.

\section{Photothermal evaluation}

The stock dispersion of BBTEHT NPs was diluted to 50,35 and $20 \mu \mathrm{g} / \mathrm{mL}$, respectively. At each concentration, a total of $1 \mathrm{~mL}$ dispersion was used in the evaluation of its photothermal effect under irradiation of a $808-\mathrm{nm}$ laser with a power density of $1.77 \mathrm{~W} / \mathrm{cm}^{2}$. Every sample was irradiated for $20 \mathrm{~min}$ and allowed to cool down to the room temperature for the next $20 \mathrm{~min}$, which was counted as one cycle. Water and gold nanorods at a concentration of $35 \mu \mathrm{g} / \mathrm{mL}$ served as the reference group and control group, respectively. The temperature was recorded using a T-type thermocouple probe (HYPO, Omega, Connecticut, USA) coupled to a thermocouple controller (NI 9211, National Instruments, Texas, USA), which was controlled by a computer.

\section{Stability evalulation of BBTEHT NPs and gold nanorods}

To test the stability of sample, and compare with gold nanorods, we irradiated 1-mL BBTEHT NPs and commercial gold nanorods under the 808-nm NIR laser with a power density of $1.77 \mathrm{~W} / \mathrm{cm}^{2}$ for $4 \mathrm{~h}$. Temperature was recorded automatically every second. The UV-Vis spectra and TEM images of the samples were measured both before and after laser irradiation to evaluate the 
changes in the transmission spectrum and morphology.

\section{Preparation of gold nanorods and cell line}

CTAB-coated Au nanorods with an aspect ratio of $3.8(10 \mathrm{~nm}$ in diameter and $38 \mathrm{~nm}$ in length) were purchascd from Strem chemicals Inc. (Massachusetts, USA). The $\mathrm{Au}$ nanorods were delivered in water suspension with a concentration of about $35 \mu \mathrm{g} / \mathrm{mL}$. To make the nanorods biocompatible, the $\mathrm{Au}$ nanorods were PEGylated with mPEG (Sigma-Aldrich, Missouri, United States). The 1- $\mu M$ mPEG solution was incubated in GNR suspension with a ratio of $1: 4$ for $6 \mathrm{~h}$ and re-suspended in PBS for future use.

Human renal cell carcinoma cells (Caki-2 cell line) were purchased from American Type Culture Collection (Virginia, USA). Caki-2 cells were cultured in Iscove's Modified Dulbecco's Medium (IMDM) supplemented with 10\% fetal bovine serum (FBS) and $500 \mu \mathrm{L}$ of Antibiotic (Antimycotic, GE Healthcare, England), and then were incubated at $37^{\circ} \mathrm{C}$ with $5 \% \mathrm{CO}_{2}$. Cultures were maintained by adding or replacing the medium cvery 3-4 days to maintain a cell density of $10^{5}-10^{6}$ cells per mL. Since Caki- 2 cell lines adhered to the surface of the culture flask during each passaging, the adherent cells were removed by replacing the culture medium by $1 \times$ Trypsin (Life Technologies, CA, USA) and putting it back in the incubator for 10 mins. Culture medium was then added to neutralise trypsin and cell counting was performed to check the cell density.

In the photothermal study, a total of $1-\mathrm{mL}$ cell suspension with a cell density of $\sim 1 \times 10^{6}$ cells $/ \mathrm{mL}$ was transferred to a well plate, where it was mixed with either 
BBTEHT NPs or PEGylated Au nanorods and incubated for another 6 hours in the incubator. The BBTEHT NPs and PEGylated Au nanorods were washed twice with the culture medium prior to mixing with the Caki-2 cells. After 6 hours, the culture medium was removed and the petridish was replaced with PBS by washing the cells gently twice in PBS to get rid of Au nanorods in the extracellular matrix. To evaluate cell death due to necrosis, trypan blue solution (Sigma-Aldrich, Missouri, USA) was added to the cell sample and was left for 10 minutes in room temperature before the observation under a bright-field microscope. Dead cells were stained blue while live cells stayed transparent, as their cytoplasm remained intact.

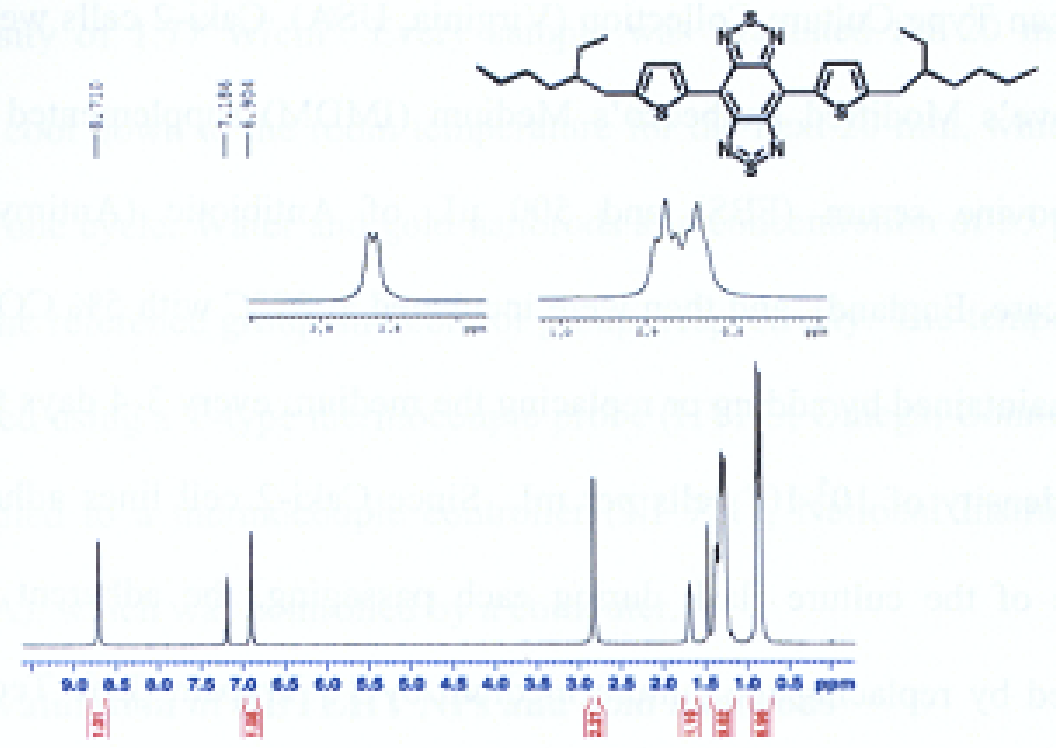

Figure 5-1. ${ }^{1} \mathrm{H}-\mathrm{NMR}\left(\mathrm{CDCl}_{3}, 400 \mathrm{MHz}\right)$ spectrum of BBTEHT. 


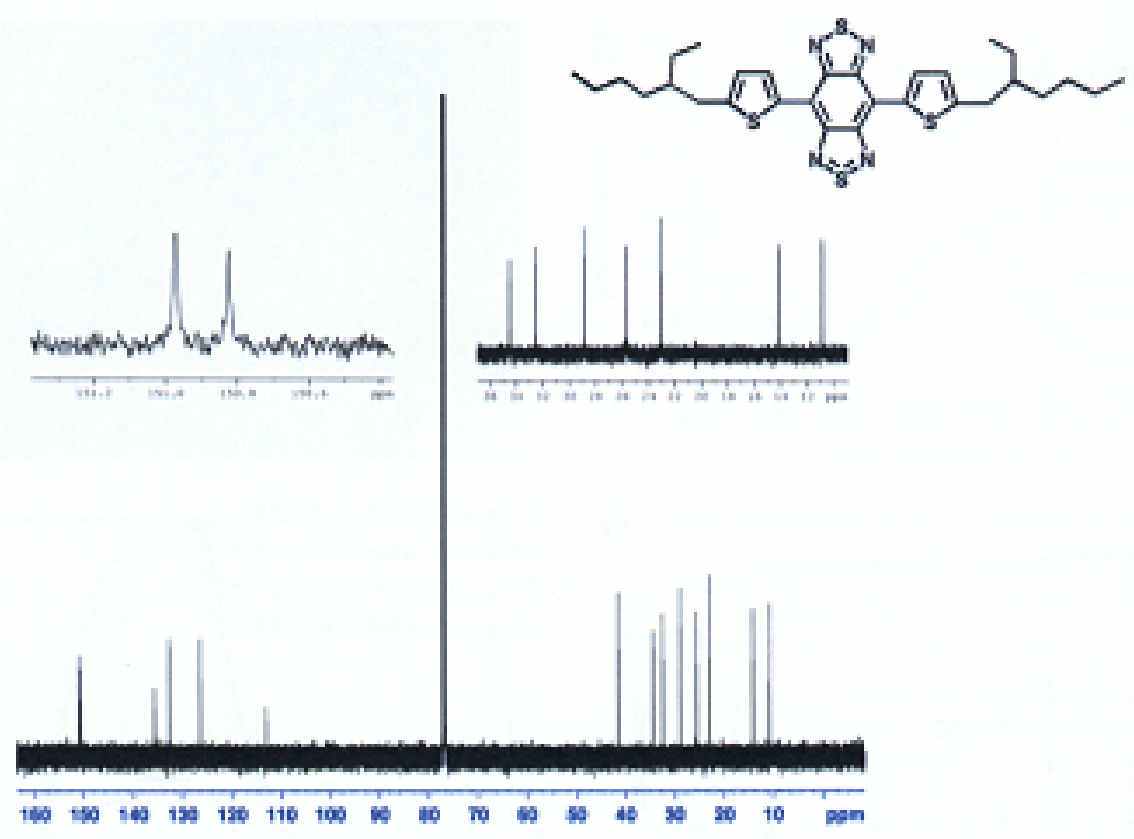

Figure 5-2. ${ }^{13} \mathrm{C}-\mathrm{NMR}\left(\mathrm{CDCl}_{3}, 100 \mathrm{MHz}\right)$ spectrum of BBTEHT.

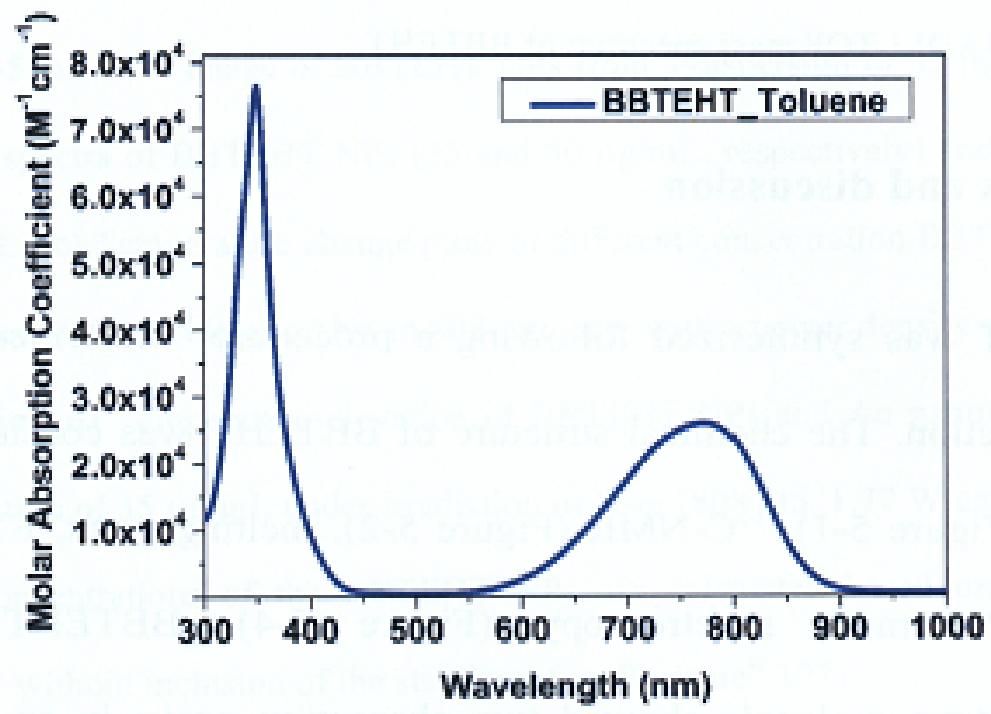

Figure 5-3. The molar absorption coefficient vs. wavelength graph of BBTEHT in toluene. 


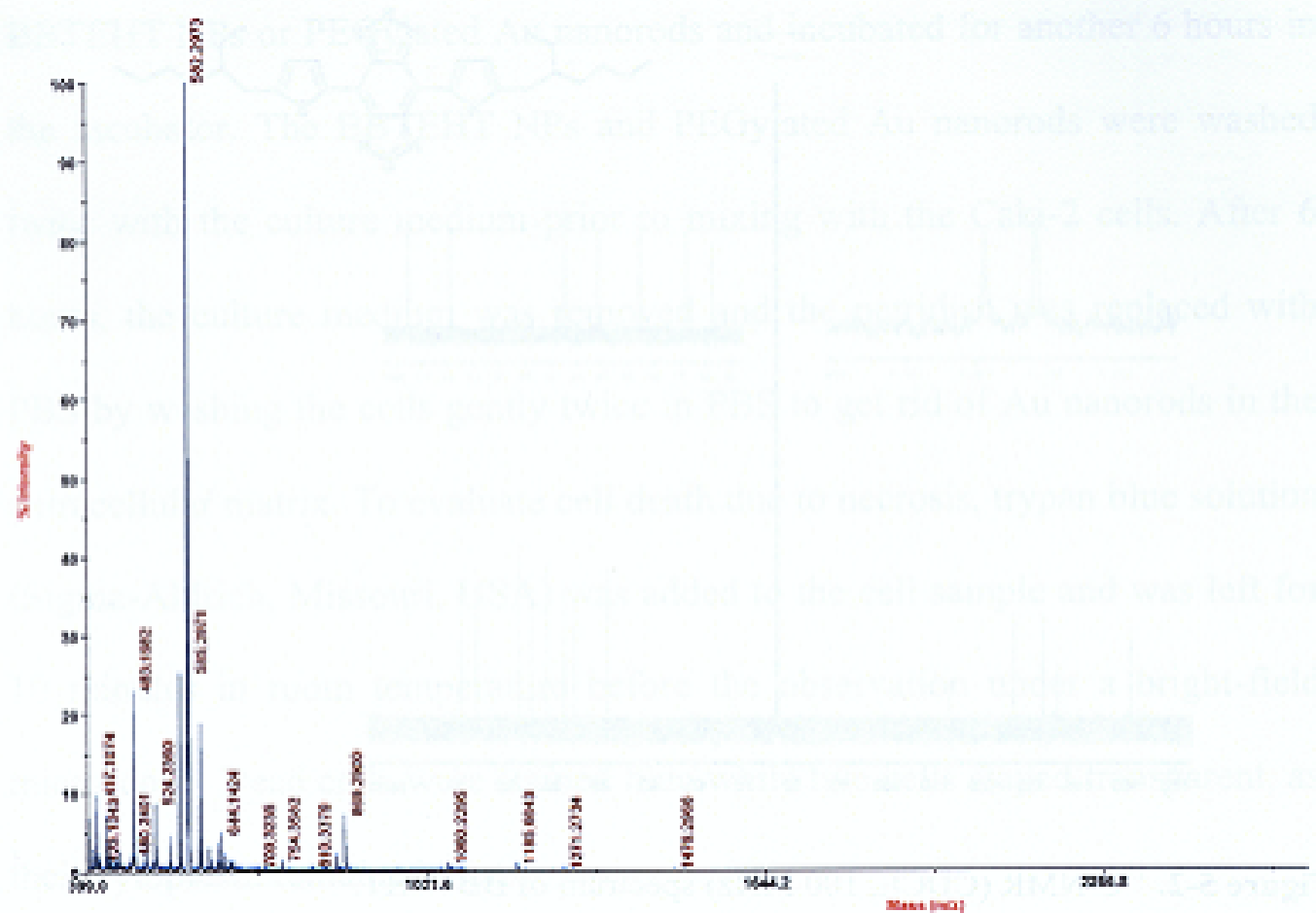

Figure 5-4. MADLI-TOF mass spectrum of BBTEHT.

\subsection{Results and discussion}

BBTEHT was synthesized following a procedure ${ }^{41}$ described in the previous section. The chemical structure of BBTEHT was confirmed by ${ }^{1} \mathrm{H}-\mathrm{NMR}$ (Figure 5-1), ${ }^{13} \mathrm{C}-\mathrm{NMR}$ (Figure 5-2), melting point, FTIR and MALDI-TOF mass spectroscopy (Figure 5-4). BBTEHT as a narrow-bandgap molecule showed two absorption peaks in an organic solvent such as toluene, one around $350 \mathrm{~nm}$ and the other at $770 \mathrm{~nm}$, with an onset around $900 \mathrm{~nm}$ (Figure 5-3). The calculated molar absorption coefficient of BBTEHT at the peak of $770 \mathrm{~nm}$ was. The absorption spectrum appeared slightly broader after BBTEHT molecules were cncapsulated with Pluronic ${ }^{\circledR} 127$ and dispersed in water (Figure 5-5 b). 
a
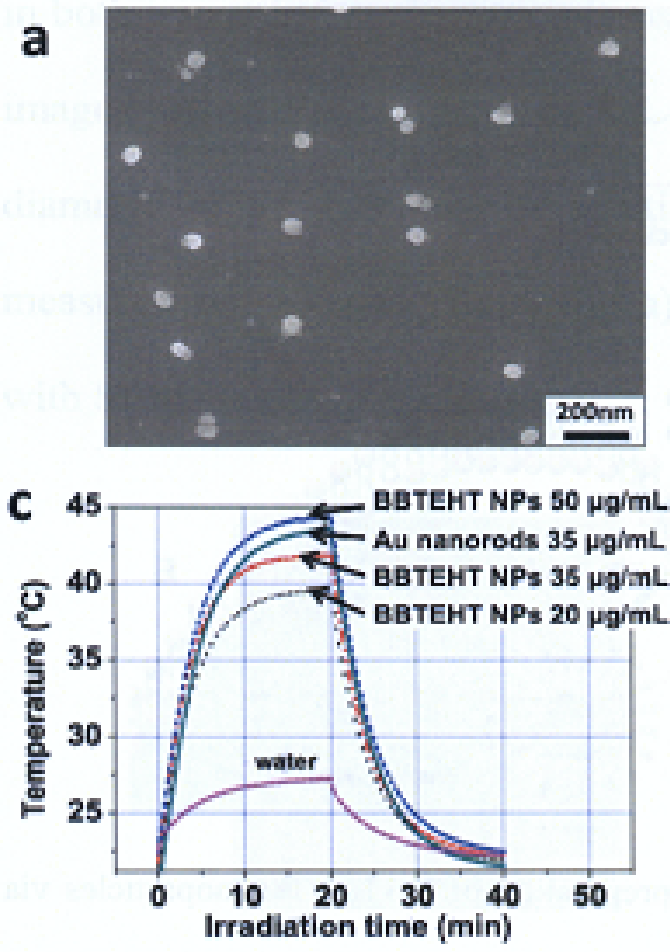
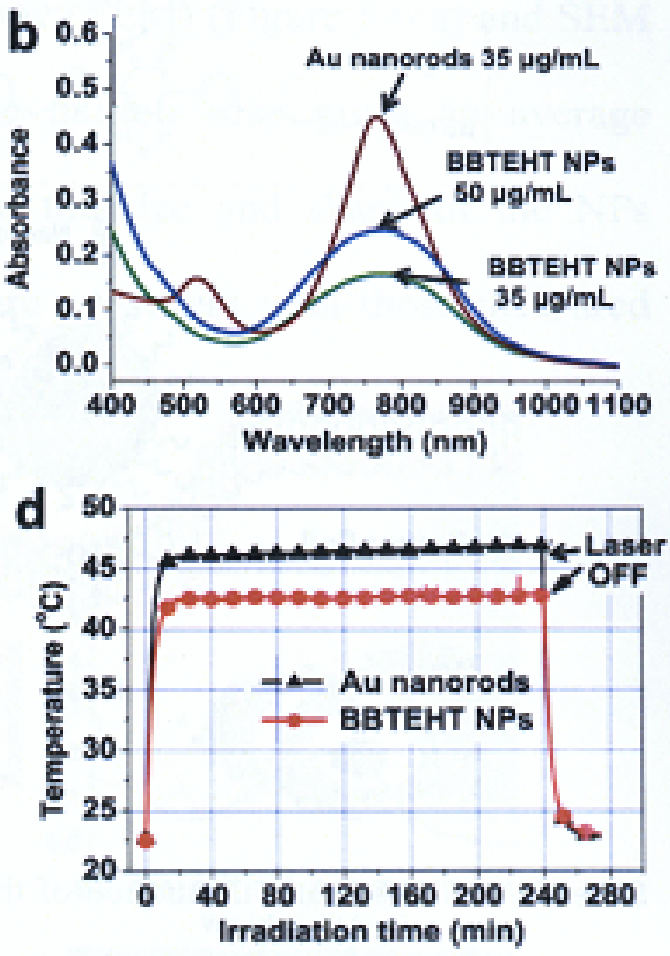

Figure 5-5. a) SEM image of BBTEHT NPs from a dispersion of $35 \mu \mathrm{g} / \mathrm{mL}$. b) UVVis-NIR spectra of BBTEHT NPs ( 35 and $50 \mu \mathrm{g} / \mathrm{mL}$, respectively) and Au nanorods $(35 \mu \mathrm{g} / \mathrm{mL}) . \mathrm{c})$ Temperature change plots of different concentration BBTEHT NPs and Au nanorods under irradiation by an $808-\mathrm{nm}$ laser with a power density of $1.77 \mathrm{~W} / \mathrm{cm}^{2}$ for 20 mins. d) Temperature elevation of BBTEHT NPs and Au nanorods both at a concentration of $35 \mu \mathrm{g} / \mathrm{mL}$ under irradiation of laser $\left(808 \mathrm{~nm}, 1.77 \mathrm{~W} / \mathrm{cm}^{2}\right)$ for $4 \mathrm{~h}$. All of the concentrations of the BBTEHT NPs are calculated based on the mass of BBTEHT without inclusion of the stabilizer (i.e. Pluronic ${ }^{\mathbb{B}}$ 127). 


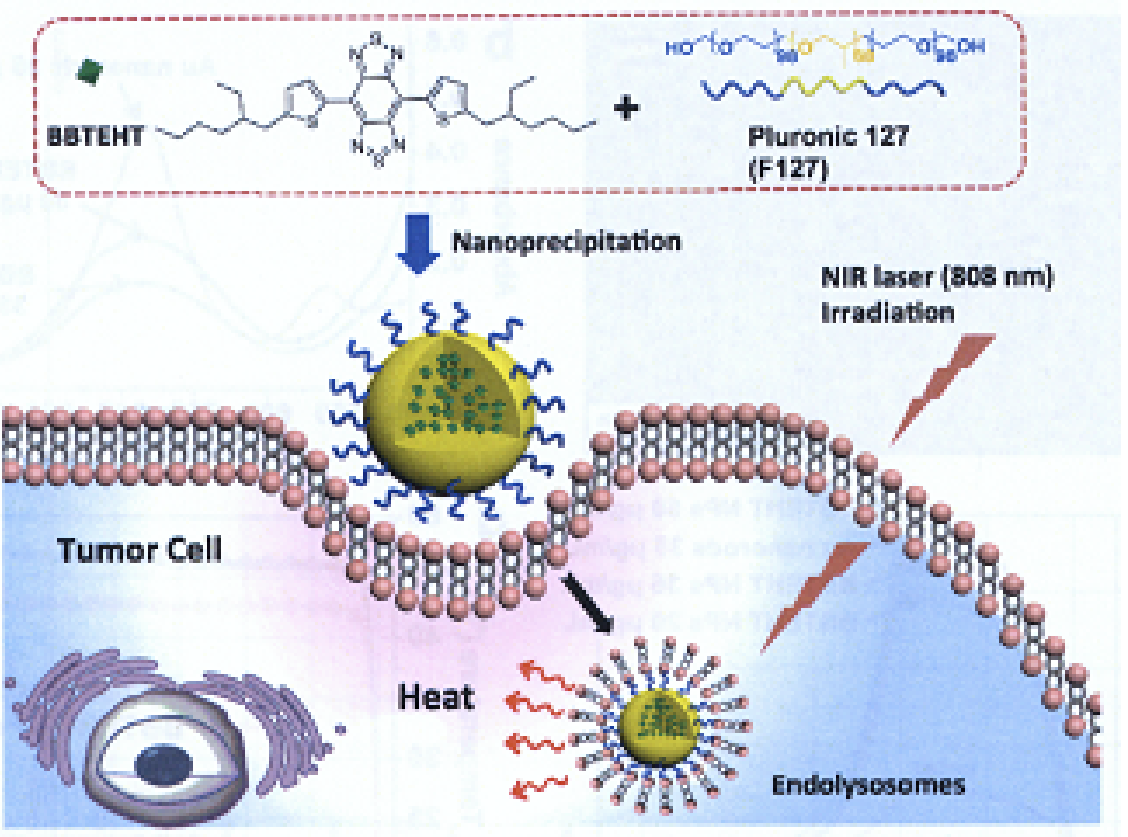

Scheme 5-1. Schematic illustration of the preparation of BBTEHT nanoparticles via nanoprecipitation process in the presence of Pluronic ${ }^{\circledR} 127$ as the stabilizer.

Scheme 5-1 shows the molecular structure of BBTEHT and a schematic presentation of the NPs formed through a nanoprecipitation process. ${ }^{42}$. At a concentration (e.g. $8.3 \mathrm{mg} / \mathrm{mL}$ ) above the critical micelle concentration $(\mathrm{CMC})$ of Pluronic ${ }^{\circledR} 127$ in water, ${ }^{40}$ self-assembly is driven by the hydrophobic interaction between the polypropylene oxide (PPO) block of Pluronic ${ }^{\circledR} 127$ and BBTEHT which collapses to form the core of the NPs. The shell of the NPs consists of PEG chains originated from Pluronic $^{\circledR} 127$ that extrude to the aqueous media to provide the colloidal stability.

A representative scanning electron microscopy (SEM) image (Figure 5-5 a) shows that the resulting spherical particles appear uniform in size and shape, although a small population of tiny NPs can also be observed 
in both transmission electron microscopy (TEM) (Figure 5-6 a) and SEM images. A statistical analysis of the particle size gives an average diameter of $53 \pm 9 \mathrm{~nm}$. In addition, the size and shape of the NPs measured with TEM (Figure 5-6 a) are consistent with those measured with SEM.

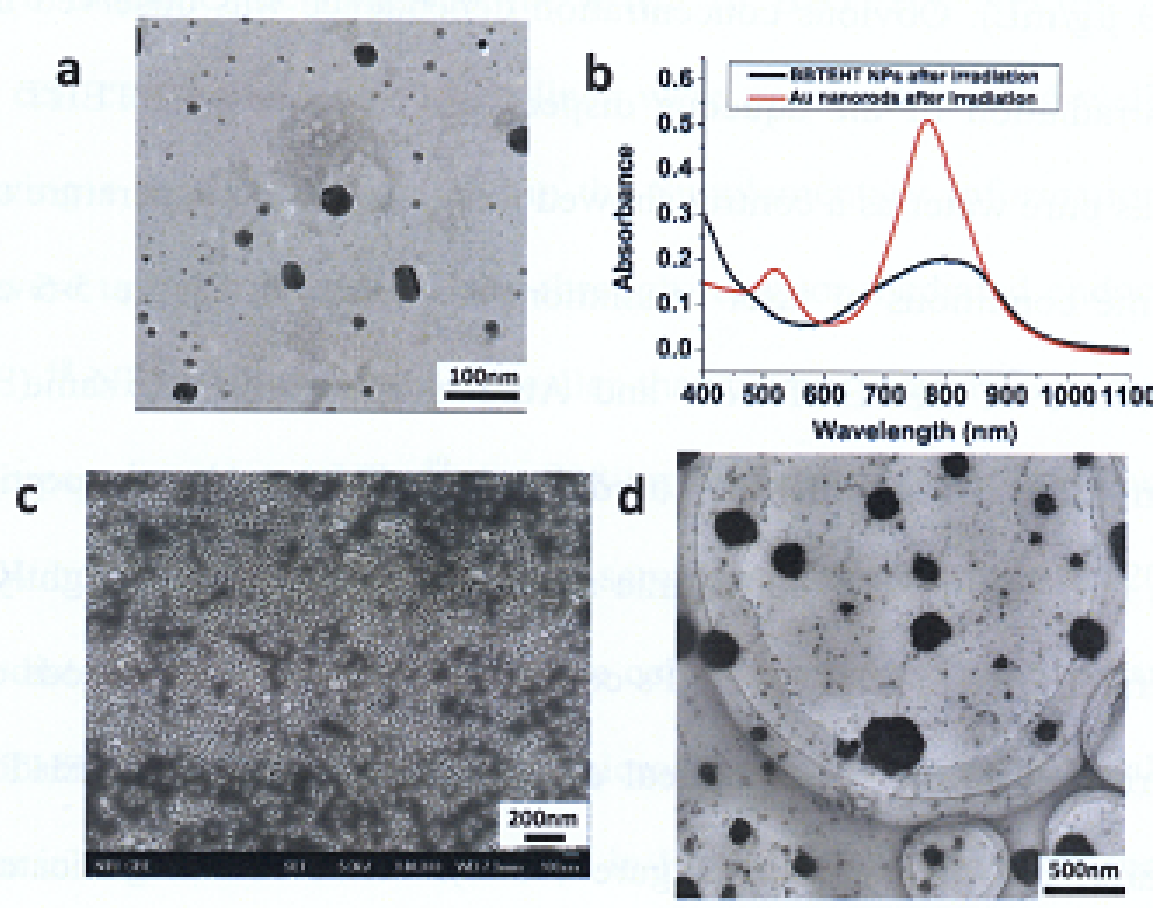

Figure 5-6. (a) TEM image of BBTEHT NPs from a dispersion of $35 \mu \mathrm{g} / \mathrm{mL}$ before laser irradiation (b) UV-VIS-NIR absorption spectra of BBTEHT NPs and Au nanorods after $4 \mathrm{~h}$ irradiations of a $808-\mathrm{nm}$ laser with a power density of $1.77 \mathrm{~W} / \mathrm{cm}^{2}$. (c-d) SEM (c) and TEM (d) images of BBTEHT NPs after 4-h irradiation of an $808-\mathrm{nm}$ laser with a power density of $1.77 \mathrm{~W} / \mathrm{cm}^{2}$.

Figure 5-5 $\mathrm{c}$ and d show the photothermal efficiency and photostability of BBTEHT NPs in comparison with commercially available Au 
nanorods with NIR absorption similar to that of BBTEHT NPs (Figure 5-5 b). The photothermal effect induced by NIR laser illumination at 808 $\mathrm{nm}$ with a power density of $1.77 \mathrm{~W} / \mathrm{cm}^{2}$ for $20 \mathrm{mins}$ in the presence of BBTEHT NPs was investigated by monitoring the temperature of $1 \mathrm{~mL}$ aqueous dispersion of BBTEHT NPs at various concentrations (20, 35, and $50 \mu \mathrm{g} / \mathrm{mL}$ ). Obvious concentration dependence was observed under laser irradiation of the aqueous dispersions containing BBTEHT NPs, whereas pure water as a control showed little change in temperature under the same conditions of laser irradiation. As shown in Figure 5-5 c, the temperature of BBTEHT NPs and Au nanorods with the same mass concentration $\left(35 \mu \mathrm{g} / \mathrm{mL}\right.$ ) increased to 42.0 and $43.6^{\circ} \mathrm{C}$, respectively, which fell within the hyperthermia region $\left(41-47^{\circ} \mathrm{C}\right)^{43}$. The slightly less temperature rise of BBTEHT NPs compared to that of Au nanorods could be attributed to the lower optical density at $808 \mathrm{~nm}$ of BBTEHT NPs compared to $\mathrm{Au}$ nanorods (Figure 5-5 c). These results indicate that BBTEHT NPs could act as an efficient photothermal agent, with photothermal conversion efficiency even higher than that of Au nanorods if their optical densities are matched.

When the NIR laser irradiation time was extended up to $4 \mathrm{~h}$, the temperature of both $\mathrm{Au}$ nanorods and BBTEHT NPs at the same concentration of $35 \mu \mathrm{g} / \mathrm{mL}$ reached a plateau at the level of $45-46{ }^{\circ} \mathrm{C}$ and $42-43{ }^{\circ} \mathrm{C}$, respectively (Figure 5-5 d). In addition, there was no obvious change of the maximum absorption peak in the NP dispersion after 
irradiation compared to the NPs before irradiation (Figure 5-6 b). Nevertheless, both the SEM and the TEM images (Figure 5-6 c and d) of BBTEHT NPs after laser irradiation show some agglomeration of the NPs, which could be due to the thermally induced gelation behavior of Pluronic $^{(B)} 127^{44-47}$

To evaluate the hyperthermic effect of BBTEHT NPs on human renal cell carcinoma, caki-2 cell lines were prepared in a 24 -well plate following a procedure described in the Supplementary Information. The NPs were uptaken by cells likely through receptor-mediated endocytosis (RME). ${ }^{48}$ NPs with a diameter smaller than $50 \mathrm{~nm}$ exhibit higher uptake as compared to larger NPs. ${ }^{49}$ An $808-\mathrm{nm}$ laser with a power density of $1.77 \mathrm{~W} / \mathrm{cm}^{2}$ was used to irradiate the sample in the well plate for $20 \mathrm{~min}$. Immediately after laser irradiation, the cells were treated with trypan blue and observed under a bright field microscope. The imaging results are shown in Figure 5-7. The dotted line in Figure 5-7 a represents the laser boundary. The images clearly show that the cells in the laser exposed section of the endocystosed NPs have undergone necrosis and hence appear blue after staining, as compared to the unexposed part, in which most cells stayed transparent. The cell death can be primarily attributed to necrosis due to the dramatic temperature increase in the first 5 mins of laser exposure based on the illustration in Figure 5-5 c. The temperature eventuaily reached the hyperthermic region and resulted in cell membrane disruption, ${ }^{50,51}$ allowing the trypan blue to enter the necrotic cell. 

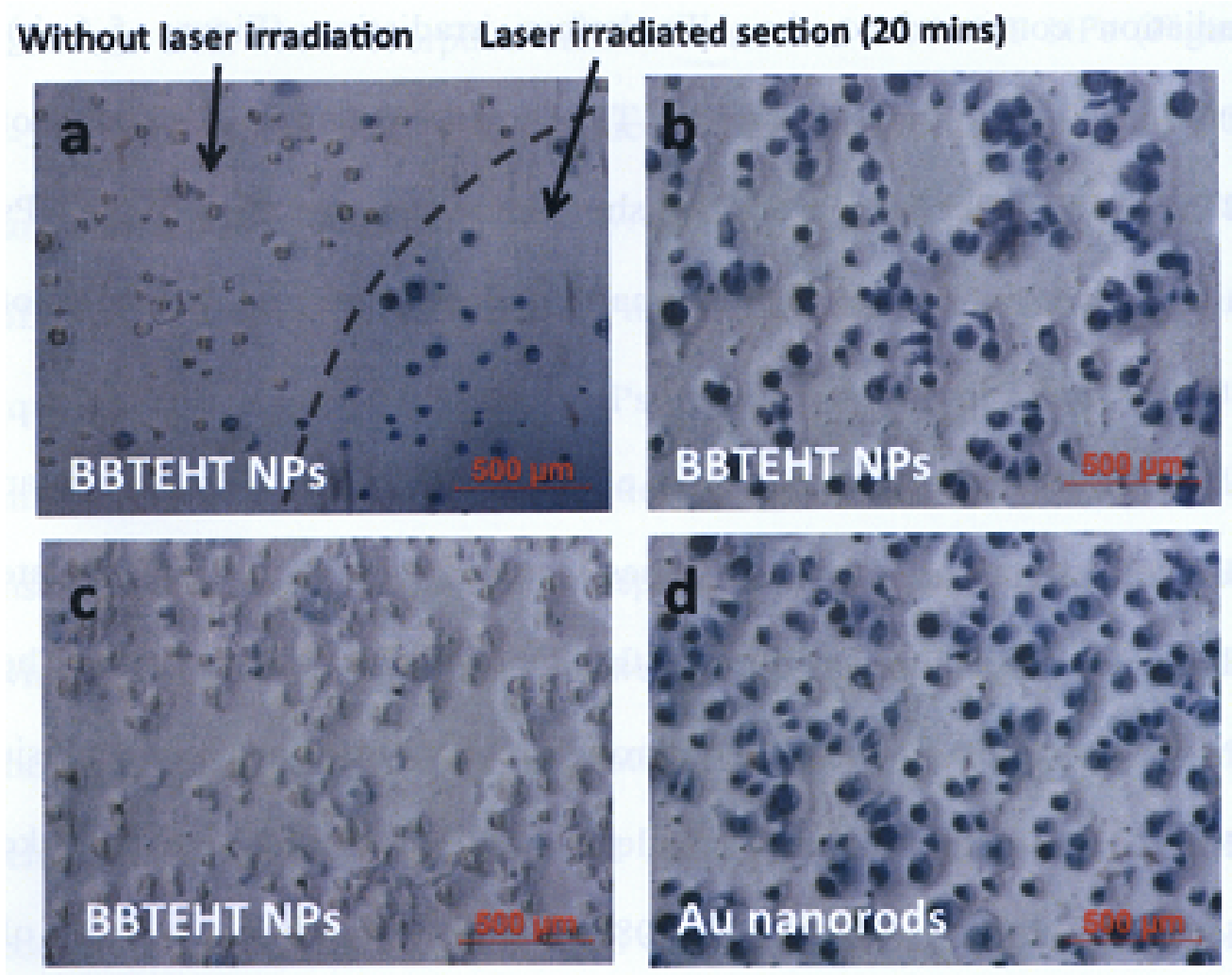

Figure 5-7. Brightfield images $(4 \times)$ of (a) caki-2 cells incubated with BBTEHT NPs in which partial area was illuminated by the laser for 20 mins. The demarkation shows laser exposed section of the cells which is stained blue due to necrosis. (b) and (d) Caki-2 cells incubated with BBTEHT NPs and Au nanorods respectively in which the entire well was illuminated by the laser for 20 mins. The concentrations of BBTEHT NPs and Au nanorods were both $35 \mu \mathrm{g} / \mathrm{mL}$. (c) Caki-2 cells incubated with BBTEHT NPs without laser illumination. 

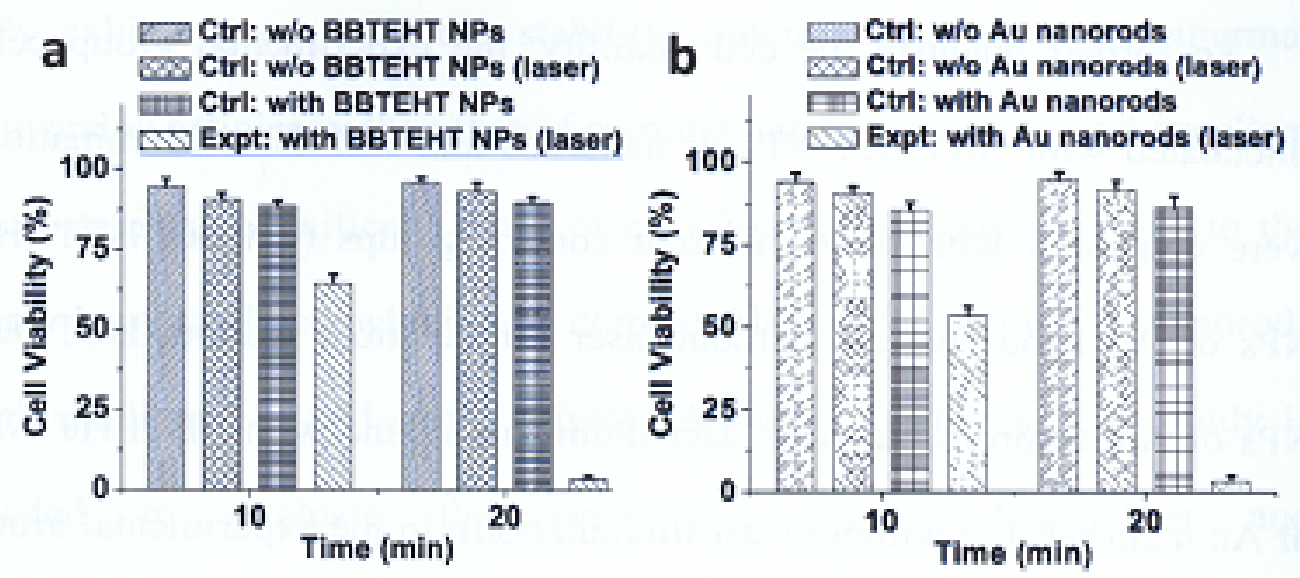

Figure 5-8. Photothermal destruction of caki-2 cells with or without BBTEHT NPs/ Au nanorods and NIR laser $\left(808 \mathrm{~nm}, 1.77 \mathrm{~W} / \mathrm{cm}^{2}\right)$. (a) Cell viability after treatment using BBTEHT NPs with 10 and 20 mins of laser exposure. (b) Cell viability after treatment using $\mathrm{Au}$ nanorods with 10 and 20 mins of laser exposure. The concentrations of BBTEHT NPs and Au nanorods were both $35 \mu \mathrm{g} / \mathrm{mL}$.

To qualitatively evaluate the photothermal therapy effect of BBTEHT NPs as compared to Au nanorods, cells were separately incubated in a 96-well plate with PEGylated Au nanorods and BBTEHT NPs at equal concentrations $(35 \mu \mathrm{g} / \mathrm{mL})$ using the same procedure. This time a smaller well size was used so that the $1.2 \mathrm{~cm}$ beam diameter of the laser could illuminate the entire well for better comparison of phototherapeutic efficacy of these two types of NPs. The results (Figure 5-7 b and d) show that cells with either BBTEHT NPs or Au nanorods induced nearly $100 \%$ necrosis in both of which all cells were stained blue. The control cells without laser irradiation docs not show any sign of necrosis. (Figure 5-7c) 
To further quantify the cell viability, the experimental group cells (incubated with BBTEHT NPs or nanorods and with laser illumination) were compared with three different control groups (without BBTEHT NPs or Au nanorods and without laser illumination, without BBTEHT NPs or Au nanorods but with laser illumination, and with BBTEHT NPs or Au nanorods but without laser illumination). In the experimental group, cells incubated with BBTEHT NPs for $6 \mathrm{~h}$ in a 96-well plate was exposed to the laser for 10 mins. At this time point, there was no apparent change in the viability of the three control groups. However, the experimental group at 10 mins showed almost $40 \%$ dead cells as compared to the control groups (Figure 5-8 a). When the irradiation time was prolonged to 20 mins there were nearly no viable cells, resulting in close to $100 \%$ cell death in the experimental group (Figure 5-8a). It can also be noticed that there were only slight changes in the cell viability in three control groups, signifying that there was no considerable cytotoxicity induced by NPs after $6 \mathrm{~h}$ of incubation. Similar results (Figure 5-8 b) were observed with PEGylated Au nanorods. These results suggest that BBTEHT NPs could be a good alternative to Au nanorods as the photothermal agent for cancer therapy.

\subsection{Conclusions}

This chapter has presented a novel photothermal therapeutic agent based on BBTEHT NPs with strong absorption in the NIR region. These 
NPs exhibit good colloidal stability, potentially higher photothermal conversion efficiency than that of commercial Au nanorods, and excellent photostability. Significant death of caki-2 cells was observed duc to the hyperthermic effect, which was comparable to that of the Au nanorods both qualitatively and quantitatively. Although further in vivo study is needed to evaluate the performance in biodistribution and pharmacokinetics, BBTEHT NP can be a promising small-molccular organic photothermal agent, expected to be more biodegradable and clinically acceptable in comparison to other photothermal agents based on inorganic materials and conjugated polymers.

\subsection{References}

1. J. T. Robinson, S. M. Tabakman, Y. Liang, H. Wang, H. S. Casalongue, D. Vinh and H. Dai, J. Am. Chem. Soc., 2011, 133, 6825-6831.

2. H. Liu, D. Chen, L. Li, T. Liu, L. Tan, X. Wu and F. Tang, Angew. Chem. Int. Ed., 2011, 50, 891-895.

3. S. Lal, S. E. Clare and N. J. Halas, Acc. Chem. Res., 2008, 41, $1842-1851$

4. D. Jaque, L. Martinez Maestro, B. Del Rosal, P. Haro-Gonzalez, A. Bcnayas, J. L. Plaza, E. Martin Rodriguez and J. Garcia Sole, Nanoscale, $2014,6,9494-9530$.

5. R. K. Kannadorai and Q. Liu, Med. Phys., 2013, 40, 103301.

6. H.-C. Huang, K. Rege and J. J. Heys, ACS nano, 2010, 4, 2892-2900. 
7. R. K. Kannadorai, G. G. Y. Chiew, K. Q. Luo and Q. Liu, Cancer Letters, $2015,357,152-159$.

8. E. Boisselier and D. Astruc, Chem. Soc. Rev., 2009, 38, 1759-1782.

9. L. Cheng, K. Yang, Y. Li, J. Chen, C. Wang, M. Shao, S. T. Lee and Z. Liu, Angew. Chem. Int. Ed. , 2011, 50, 7385-7390.

10. W. Dong, Y. Li, D. Niu, Z. Ma, J. Gu, Y. Chen, W. Zhao, X. Liu, C. Liu and J. Shi, Adv. Mater., 2011, 23, 5392-5397.

11. Y. Xia, W. Li, C. M. Cobley, J. Chen, X. Xia, Q. Zhang, M. Yang, E. C. Cho and P. K. Brown, Acc. Chem. Res., 2011, 44, 914-924.

12. L. Dykman and N. Khlebtsov, Chem. Soc. Rev., 2012, 41, 2256-2282.

13. L. Nie, M. Chen, X. Sun, P. Rong, N. Zheng and X. Chen, Nanoscale, 2014, 6, 1271-1276.

14. K. Yang, L. Feng, X. Shi and Z. Liu, Chem. Soc. Rev., 2013, 42, $530-547$.

15. C. Li, S. Bolisetty, K. Chaitanya, J. Adamcik and R. Mezzenga, Adv. Mater., 2013, 25, 1010-1015.

16. U. Rocha, K. U. Kumar, C. Jacinto, I. Villa, F. Sanz-Rodriguez, C. Iglesias de la Cruz Mdel, A. Juarranz, E. Carrasco, F. C. van Veggel, E. Bovero, J. G. Sole and D. Jaque, Small, 2014, 10, 1141-1154.

17. Q. Tian, M. Tang, Y. Sun, R. Zou, Z. Chen, M. Zhu, S. Yang, J. Wang, J. Wang and J. Hu, Adv. Mater., 2011, 23, 3542-3547.

18. Q. Tian, F. Jiang, R. Zou, Q. Liu, Z. Chen, M. Zhu, S. Yang, J. Wang, J. Wang and J. Hu, ACS nano, 2011, 5, 9761-9771. 
19. Z. Chen, Q. Wang, H. Wang, L. Zhang, G. Song, L. Song, J. Hu, H. Wang, J. Liu, M. Zhu and D. Zhao, Adv. Mater., 2013, 25, 2095-2100.

20. T. N. Lambert, N. L. Andrews, H. Gerung, T. J. Boyle, J. M. Oliver, B. S. Wilson and S. M. Han, Small, 2007, 3, 691-699.

21. C. Lee, H. Kim, C. Hong, M. Kim, S. S. Hong, D. H. Lee and W. I. Lee, J. Mater. Chem., 2008, 18, 4790-4795.

22. S. Sharifi, S. Behzadi, S. Laurent, M. L. Forrest, P. Stroeve and M. Mahmoudi, Chem. Soc. Rev., 2012, 41, 2323-2343.

23. L. Cheng, K. Yang, Q. Chen and Z. Liu, ACS nano, 2012, 6, $5605-5613$

24. Z. Zha, X. Yue, Q. Ren and Z. Dai, Adv. Mater., 2013, 25, 777-782.

25. Y. Liu, K. Ai, J. Liu, M. Deng, Y. He and L. Lu, Adv. Mater., 2013, 25, 1353-1359.

26. J. Yang, J. Choi, D. Bang, E. Kim, E. K. Lim, H. Park, J. S. Suh, K. Lce, K. H. Yoo, E. K. Kim, Y. M. Huh and S. Haam, Angew. Chem. Int. $E d ., 2011, \mathbf{5 0}, 441-444$.

27. L. Cheng, W. He, H. Gong, C. Wang, Q. Chen, Z. Cheng and Z. Liu, Adv. Funct. Mater., 2013, 23, 5893-5902.

28. M. Zheng, C. Yue, Y. Ma, P. Gong, P. Zhao, C. Zheng, Z. Sheng, P. Zhang, Z. Wang and L. Cai, ACS nano, 2013, 7, 2056-2067.

29. X. He, X. Wu, K. Wang, B. Shi and L. Hai, Biomaterials, 2009, 30, $5601-5609$. 
30. S. Zonghai, H. Dehong, X. Miaomiao, H. Meng, G. Ping and C. Lintao, Nano-Micro Lett., 2013, 5, 145-150.

31. C. S. Jin, J. F. Lovell, J. Chen and G. Zheng, ACS nano, 2013, 7, 2541-2550.

32. G. Qian, B. Dai, M. Luo, D. Yu, J. Zhan, Z. Zhang, D. Ma and Z. Y. Wang, Chem. Mater., 2008, 20, 6208-6216.

33. J. Huber, C. Jung and S. Mecking, Macromolecules, 2012, 45, 7799-7805.

34. J. D. Yuen, M. Wang, J. Fan, D. Sheberla, M. Kemei, N. Banerji, M. Scarongella, S. Valouch, T. Pho, R. Kumar, E. C. Chesnut, M. Bendikov and F. Wudl, J. Polym. Sci., Part A: Polym. Chem., 2015, 53, 287-293.

35. J. Fan, J. D. Yuen, M. Wang, J. Seifter, J.-H. Seo, A. R. Mohebbi, D. Zakhidov, A. Heeger and F. Wudl, Adv. Mater., 2012, 24, 2186-2190.

36. J. Fan, J. D. Yuen, W. Cui, J. Seifter, A. R. Mohebbi, M. Wang, H. Zhou, A. Heeger and F. Wudl, Adv. Mater., 2012, 24, 6164-6168.

37. T. T. Steckler, X. Zhang, J. Hwang, R. Honeyager, S. Ohira, X. H. Zhang, A. Grant, S. Ellinger, S. A. Odom, D. Sweat, D. B. Tanner, A. G. Rinzler, S. Barlow, J. L. Bredas, B. Kippelen, S. R. Marder and J. R. Reynolds, J. Am. Chem. Soc., 2009, 131, 2824-2826.

38. T. T. Steckler, K. A. Abboud, M. Craps, A. G. Rinzler and J. R. Reynolds, Chem. Commun., 2007, 4904-4906.

39. A. Pitto-Barry and N. P. E. Barry, Polym. Chem., 2014, 5, 3291-3297. 
40. A. V. Kabanov, E. V. Batrakova and V. Y. Alakhov, J. Control. Release, 2002, 82, 189-212.

41. T. L. Tam, H. Li, F. Wei, K. J. Tan, C. Kloc, Y. M. Lam, S. G. Mhaisalkar and A. C. Grimsdale, Org. Lett., 2010, 12, 3340-3343.

42. S. Huang, S. Liu, K. Wang, C. Yang, Y. Luo, Y. Zhang, B. Cao, Y. Kang and M. Wang, Nanoscale, 2015, 7, 889-895.

43. T. F. Cabada, C. S. L. de Pablo, A. M. Serrano, F. D. Guerrero, J. J. S. Olmedo and M. R. Gomez, Int. J. Nanomedicine, 2012, 7, 1511-1523.

44. S. M. Shishido, A. B. Seabra, W. Loh and M. Ganzarolli de Oliveira, Biomaterials, 2003, 24, 3543-3553.

45. P. Alexandridis and T. Alan Hatton, Colloids Surf., A, 1995, 96, 1-46.

46. M. Malmsten, Soft Matter, 2006, 2, 760-769.

47. S. Y. Lee, Y. Lee, J. E. Kim, T. G. Park and C.-H. Ahn, J. Mater. Chem., 2009, 19, 8198-8201.

48. S. Mukherjee, R. N. Ghosh and F. R. Maxfield, Physiol. Rev., 1997, 77, 759-803.

49. D. B. Chithrani, Molec. Membrane Biol., 2010, 27, 299-311.

50. B. V. Harmon, A. M. Corder, R. J. Collins, G. C. Gobé, J. Allen, D. J. Allan and J. F. R. Kerr, Int. J. Rad. Biol., 1990, 58, 845-858.

51. J. Zhou, X. Wang, L. Du, L. Zhao, F. Lei, W. Ouyang, Y. Zhang, Y. Liao and J. Tang, Mol. Med. Rep., 2011, 4, 187-191. 


\section{Chapter 6 A dual-functional benzobisthiadiazole derivative as an effective theranostic agent for}

near-infrared photoacoustic imaging and photothermal therapy

In previous chapter, despite the benzobisthiadiazole derivative has been demonstrated with high photothermal conversion efficiency for cancer therapy. It is important to explore a theranostic agent, which can serve as a diagnostic and therapeutic agent. Though some inorganic dual functional theranostic agents such as gold nanoparticles have been explored, most of them still suffer from poor biodegradability and biocompatibility. In this chapter, I will present a theranostic agent based on a narrow-bandgap small molecule, benzo[1,2-c;4,5-c']bis[1,2,5]thiadiazole-4,7-bis(9,9-dioctyl-9H-fluoren-2-yl)thi ophene (denoted as BBT-2FT), with strong absorption of near infrared (NIR) light. Colloidal nanoparticles composed of BBT-2FT show photoacoustic signal intensity 10 times higher than that of blood, and high photothermal conversion efficiency $(\eta=40 \%)$ under irradiation of $800-\mathrm{nm}$ laser light that kills over $90 \%$ HeLa cells in $10 \mathrm{~min}$. This work has been published in Journal of Materials Chemistry B in 2016. 


\subsection{Introduction}

Theranostic, defined as a material that combines the modalities of therapy and diagnosis, has attracted great attention since it was coined in $2002^{1-3}$. Before starting treatments to various diseases such as cancer, it is important to monitor and understand the status and locations of the disease. In contrast to using two separate agents, theranostic aims to incorporate various agents into one material, in order to realize imaging and therapy simultaneously. For instance, some of the therapeutic strategies developed for different types of cancer treatments, such as nucleic therapy, chemotherapy, photothermal therapy, photodynamic therapy, and radiation therapy, can be functionalized with imaging strategies, such as magnetic resonance imaging (MRI), nuclear imaging (PET/CT), photoacoustic imaging, and fluorescence imaging ${ }^{4-10}$.

Among a variety of diagnosis techniques, photoacoustic tomography (PAT) is a promising medical imaging technique that combines rich optical contrast and scalable high ultrasonic resolution in a single modality ${ }^{11-14}$. PAT can provide deeper tissue imaging than other pure optical imaging modalities (Fluorescence microscopy, Raman microscopy, Optical coherence tomography, etc.). In PAT, short (nanosecond) non-ionising laser pulses are absorbed by the biological tissue leading to transient thermal expansion of the tissue and subsequent ultrasound emission. The generated ultrasound waves are acquired by ultrasound 
transducer to form a photoacoustic image. PAT has been proven to be a promising technique for imaging biological features from organelle to organs ${ }^{14}$. The application of PAT includes, but not limited to, small animal brain imaging, breast cancer imaging, monitoring of vascularisation, tumor angiogenesis, blood oxygenation, total haemoglobin concentration, etc. ${ }^{15-21}$

Biological tissues have relatively low absorption in the near infrared (NIR) region ${ }^{22,23}$. Therefore, NIR wavelength light has been used for deep tissue PAT imaging. Several exogenous contrast agents with high absorption in the NIR region have been synthesized and used to enhance the contrast for deep tissue PAT imaging ${ }^{24}$. Noble metallic nanoparticles with different shapes such as gold $(\mathrm{Au})$ nanoshells ${ }^{25}$, nanorods ${ }^{26}$, and nanocages $^{27,28}$, nanobeacons ${ }^{29,30}$ have been widely used as contrast agents for PAT imaging. Other inorganic NPs, such as single-walled carbon nanotubes (SWCNTs) $)^{31,32}$ and copper sulphide (CS) $\mathrm{NPs}^{33}$, served as good contrast agents for deep PAT imaging. Quantum dots were also used as multi-modal contrast agent in photoacoustic and photothermal imaging ${ }^{34}$. Nevertheless, the biodegradability and long-term toxicity of these inorganic materials remain an issue for their use in clinical trials. Organic dyes such as IRDye-800, and indocyanine green (ICG) ${ }^{35,36}$ have better biocompatibility and drug-delivery capability. But they suffer from relatively small optical absorption cross-section, and could be easily removed by the renal system due to their relatively small size $(<10 \mathrm{~nm})$. 
Dye-doped porphysome organic NPs have been reported for PAT imaging $^{37}$, but their synthesis process is also complicated and have relatively large diameter $(>100 \mathrm{~nm})$ which could be removed by macrophage system mostly by the liver and spleen. Recently, a biocompatible polypyrrole organic NPs ( $\sim 46 \mathrm{~nm}$ in diameter) has been demonstrated for deep tissue imaging in PAT system ${ }^{38}$.

Among many strategies for cancer treatment, photothermal therapy has been widely used due to its advantages such as high specificity, minimal invasiveness, low toxicity to normal tissues, and excellent anti-cancer efficacy. ${ }^{39-41}$ As a consequence, many efforts have been devoted to explore various theranostic nanomaterials, especially combining NIR photoacoustic imaging and photothermal therapy due to the deep penetration of tissues, high specificity, minimal invasiveness, selective damage and excellent anti-cancer efficacy. Though many theranostic platforms $\mathrm{s}^{42-46}$ have been established as the combining form of the therapeutic agents and imaging agents, the two agents may slowly dissociate from the carrier at different rates during the circulation in blood, leading to quite different biodistribution and pharmacokinetics. Thus, it is important to explore a photothermal agent, which can serve as a contrast agent itself. To that end, it is necessary to incorporate photoacoustic imaging and photothermal therapy into one agent to realize diagnosis and therapy together. Some dual-modal theranostic materials have been reported, such as combining photoacoustic imaging and PTT using 
inorganic nanoparticles (NPs) $)^{34,47-51}$ and conjugated polymers ${ }^{52}$. However, these inorganic materials incorporating two functions into one material still suffer from poor biocompatibility and biodegradability.

Benzo[1,2-c;4,5-c']bis[1,2,5]thiadiazole (BBT) based derivatives are well-known narrow-bandgap building blocks for organic optoelectronic devices $^{53-59}$. Taking the advantage of the strong light absorption of BBT derivatives in near NIR window that can benefit deep tissue imaging and therapy, Wang and coworkers $^{60}$ recently reported that colloidal nanoparticles composed of a small molecular BBT derivative (denoted as BBTEHT) showed high photothermal conversion efficiency and robust photostability compared to gold NPs ${ }^{61,62}$ for effective treatment of cancer cells. We expected that the impressive photothermal performance of BBT derivatives would also enable their applications as contrast agents for photoacoustic imaging, leading to a new type of theranostic agent that integrate the functionality of both photothermal therapy and photoacoustic imaging.

In this chapter, I present such a theranostic agent based on a small-molecular BBT derivative, benzo[1,2-c;4,5-c']bis $[1,2,5]$ thiadiazole-4,7-bis(9,9-dioctyl-9H-fluoren-2-yl)thiophene (denoted as BBT-2FT). BBT unit, due to its hypervalent sulfur atoms stabilized in quinoidal structures in a conjugated backbone, is a strong electron accepting unit that ensures strong NIR absorption of BBT-2FT in the therapeutic optical window. The biradical nature of BBT unit as well as 
the presence of thermally accessible triplet states may also contribute to the NIR absorption. ${ }^{63}$ The BBT-2FT NPs prepared through nanoprecipitation in the presence of block polymer PEG- $b$-PCL possess excellent stability and biocompatiblity. The NPs not only demonstrated significant photothermal conversion under $808 \mathrm{~nm}$ laser irradiation, but also served as photoacoustic imaging contrast agent and provided strong photoacoustic signal compared to blood. With the treatments of BBT-2FT agents and laser, 90\% HeLa cells could be killed in $10 \mathrm{~min}$.

\subsection{Materials and Methods.}

\subsubsection{Materials and characterization}

All reagents and chemicals were purchased form Sigma Aldrich and used as received. All reactions were carried out under $\mathrm{N}_{2}$ atmosphere unless noted specifically. NMR spectra were recorded by Avance 300 NMR. Transmission electron microscope (TEM) measurements were carried out on a Carl Zeiss Libra 120 electron microscope operating at an acceleration voltage of $120 \mathrm{kV}$. UV-vis absorption spectra were recorded on a UV-2450 (SHIMADZU, Japan) spectrophotometer. Steady-state photoluminescence emission spectra were recorded with LS 55 (PerkinElmer, USA) fluorescence spectrometer. Fluorescent quantum yield was measured by Edinburgh fluorescence spectrometer FLS980. The particle size was measured with a dynamic light scattering (DLS) instrument, Zetasizer Nano ZS (Malvern, UK). 


\subsubsection{Methods}

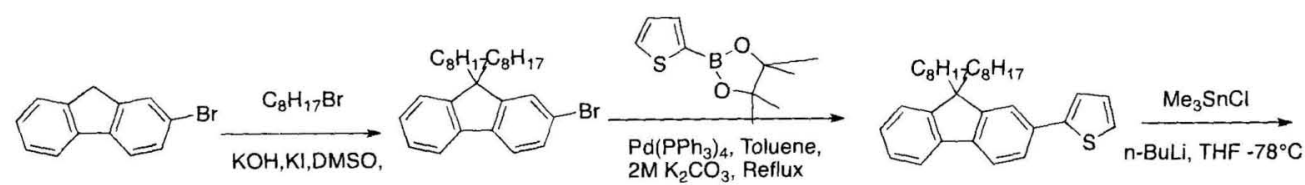

1

2

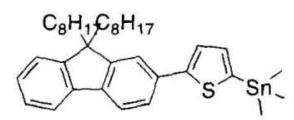

3

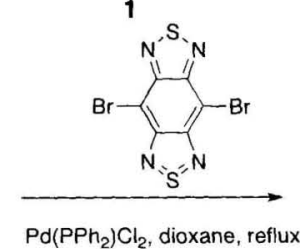

$\mathrm{Pd}\left(\mathrm{PPh}_{2}\right) \mathrm{Cl}_{2}$, dioxane, reflux

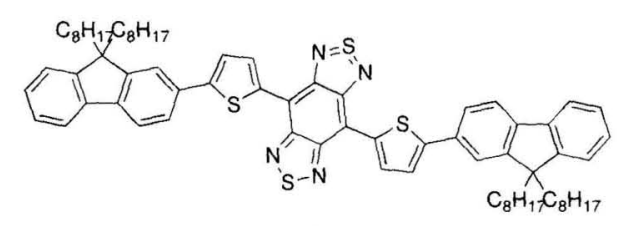

4

Scheme 6-1. Synthetic route of BBT-2FT molecule.

\section{2-Bromo-9, 9-dioctylfluorene (1).}

1-Bromooctane ( $8.5 \mathrm{~g}, 44.0 \mathrm{mmol})$ was added by using a syringe to a mixture of 2-bromofluorene $(4.5 \mathrm{~g}, 20.0 \mathrm{mmol})$, potassium iodide $(330 \mathrm{mg}, 2 \mathrm{mmol})$ and $\mathrm{KOH}(11.2 \mathrm{~g}, 200.0 \mathrm{mmol})$ in DMSO $(20 \mathrm{~mL})$. The solution was stirred at $60^{\circ} \mathrm{C}$ overnight. The mixture was poured into water $(200 \mathrm{~mL})$, and then was extracted three times with ethyl acetate. The combined organic layers were washed with brine and dried over anhydrous $\mathrm{MgSO}$. The solvent was removed under reduced pressure. The crude product was purified by column chromatography by using hexane as eluent to yield oil $(8.3 \mathrm{~g}, 89 \%) .{ }^{1} \mathrm{H}-\mathrm{NMR}(300 \mathrm{MHz}$, $\left.\mathrm{CDCl}_{3}\right): \mathrm{d}=7.65(\mathrm{~m}, 1 \mathrm{H}), 7.54(\mathrm{~m}, 1 \mathrm{H}), 7.44(\mathrm{~m}, 2 \mathrm{H}), 7.31(\mathrm{~m}, 3 \mathrm{H}), 1.92(\mathrm{~m}$, $4 \mathrm{H}), 0.97-1.24(\mathrm{~m}, 20 \mathrm{H}), 0.81(\mathrm{t}, \mathrm{J}=7.2 \mathrm{~Hz}, 6 \mathrm{H}), 0.58 \mathrm{ppm}(\mathrm{m}, 4 \mathrm{H})$. 
2-bromo-9,9-dioctylfluorene $(1 \mathrm{mmol})$ and the Thiophene-2-boronic acid bis(pinacol) ester $(1.2 \mathrm{mmol})$ in the presence of $3 \mathrm{~mol} \% \mathrm{Pd}\left(\mathrm{PPh}_{3}\right)_{4}$ in an aqueous mixture of Toluene $/ 2 \mathrm{M} \mathrm{K} 2 \mathrm{CO} 3=12 \mathrm{ml}: 3 \mathrm{ml}$. The solution was stirred and refluxed for $12 \mathrm{~h}$ under an inert atmosphere. The mixture was poured into water and extracted with diethyl ether. The organic extracts were washed with brine and dried over magnesium sulfate. The solvent was removed, and the residue was purified by column chromatography using hexanes as the eluent. The yield is $69 \%$.

\section{(5-(9,9-dioctyl-9H-fluoren-2-yl)thiophen-2-yl)trimethylstannane (3)}

To a solution of 2-(9,9-dioctyl-9H-fluoren-2-yl)thiophene $(1.77 \mathrm{~g}, 4.1 \mathrm{mmol})$ in $30 \mathrm{~mL}$ of $\mathrm{THF}$ at $-78{ }^{\circ} \mathrm{C}$ was added $n$-BuLi $(1.6 \mathrm{M}$ in hexane, $2.82 \mathrm{~mL}, 4.5$ mmol) dropwise. After the mixture was stirred for $1 \mathrm{~h}$ at $-78^{\circ} \mathrm{C}$, then return to 0 ${ }^{\circ} \mathrm{C}$ for $1 \mathrm{~h}$, and then at $-78{ }^{\circ} \mathrm{C}$ trimethylstannyl chloride $(4.5 \mathrm{ml}, 4.5 \mathrm{mmol})$ was added to the mixture. Then it was slowly warmed to room temperature and stirred for another $12 \mathrm{~h}$. The mixture was poured into water and extracted with dichloromethane. The organic extracts were dried over MgSO4. Upon evaporation of the solvent, the crude product was obtained for the next step without further purification.

\section{BBT-2FT (4)}

In a two neck $100 \mathrm{ml}$ round bottom flask, to the mixture of 4,8-dibromo-BBT $(0.14 \mathrm{~g}, 0.40 \mathrm{mmol})$, of $\mathrm{Pd}\left(\mathrm{PPh}_{3}\right)_{2} \mathrm{Cl}_{2}(28 \mathrm{mg} 10 \mathrm{~mol} \%)$ in $8 \mathrm{~mL}$ of anhydrous 
dioxane was added trimethyl(4-(2-octyldodecyl)thiophen-2-yl)stannane ( $80 \%$ purity) $(0.909 \mathrm{~g}, 1.0 \mathrm{mmol})$. The mixture was bubbled with Argon for $10 \mathrm{~min}$ and refluxed for 20 hours. Then the solvent was removed under reduced pressure, and the crude product was purified by column chromatography, by using hexane as the eluent to remove the unreacted tin compound, using $\mathrm{DCM} /$ hexane $=1 / 9$ to isolate the BBTFT as a dark green solid $(400 \mathrm{mg}, 88 \%)$.

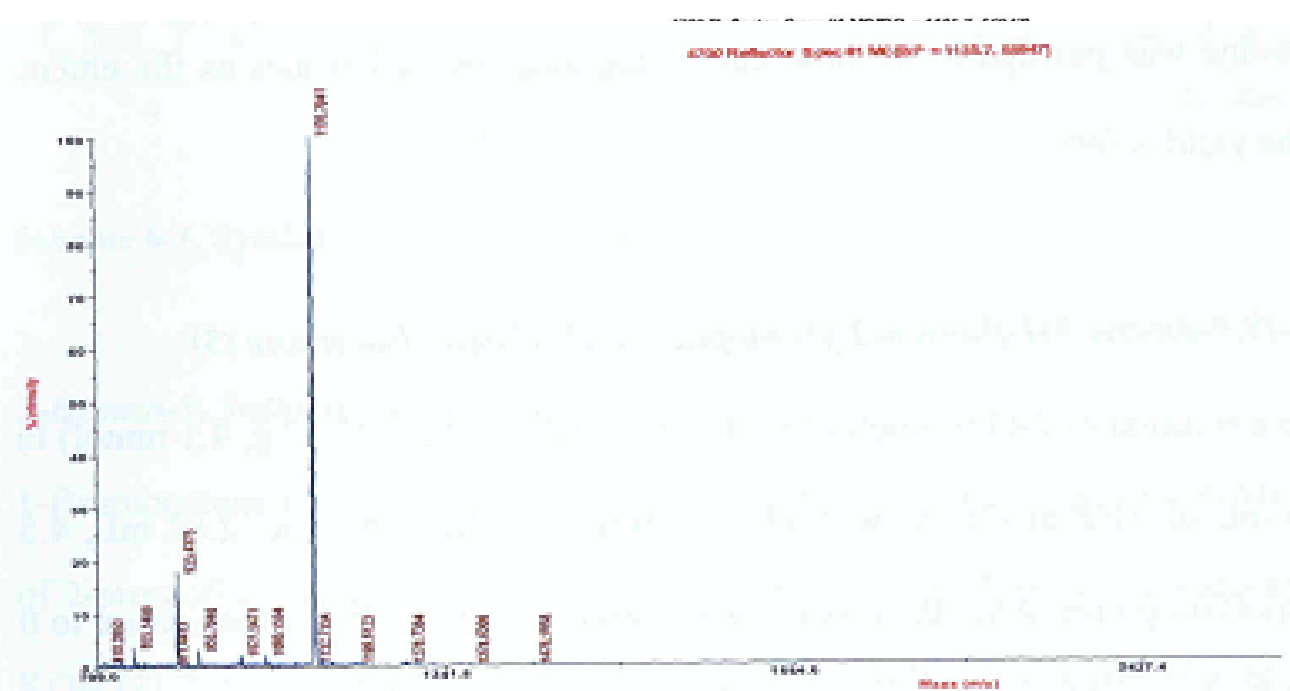

Figure 6-1. MADLI-TOF mass spectrum of BBT-2FT.

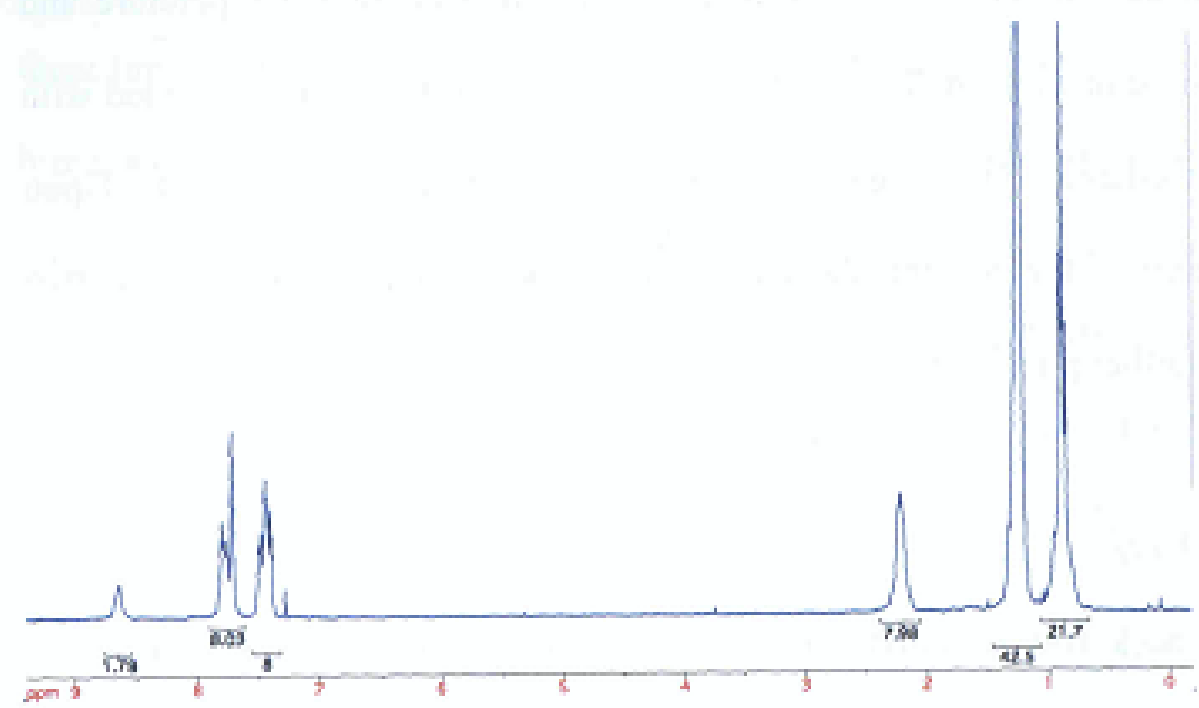

Figure 6-2. ${ }^{1} \mathrm{H}-\mathrm{NMR}\left(300 \mathrm{MHz}, \mathrm{CDCl}_{3}\right)$ spectrum of $\mathrm{BBT}-2 \mathrm{FT}$. 


\section{Synthesis of PEG-b-PCL:}

Prepared $\beta$-capeolactone (5 g) and MePEG (2.5 g) were dissolved in $5 \mathrm{ml}$ anhydrous toluene in $100 \mathrm{ml}$ dried schelink tube. The equipment was degassed and filled nitrogen three times. Then Stannous 2-ethylhexanoate (1 droplet) was added into the solution. The refluxing mixture was stirred at $110^{\circ} \mathrm{C}$ for $24 \mathrm{~h}$. After cooling to room temperature, the mixtures were precipitated in ethyl ether three times to abtain white powder. The molecule weight was calculated by GPC and ${ }^{1} \mathrm{H}-\mathrm{NMR}$.

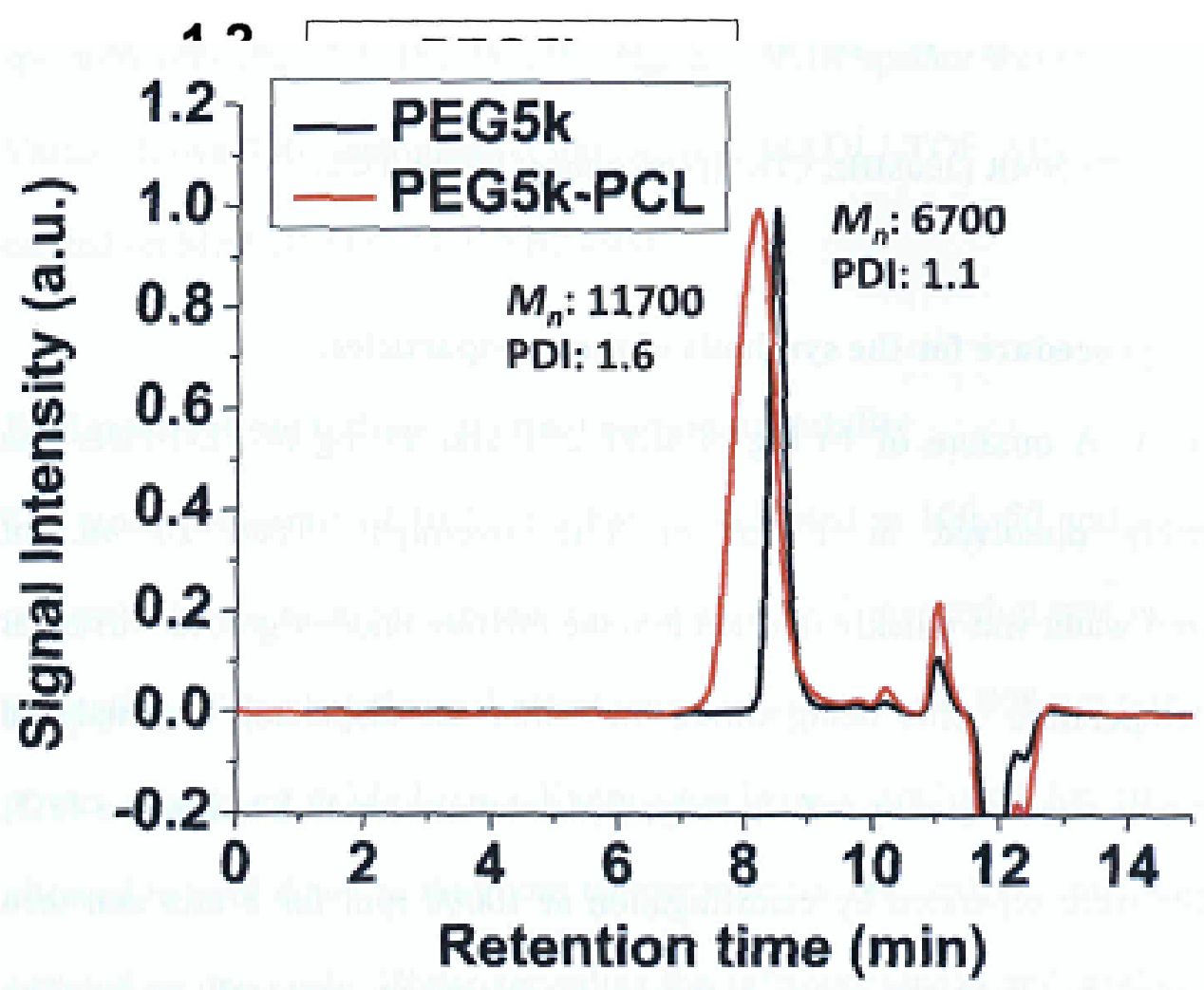




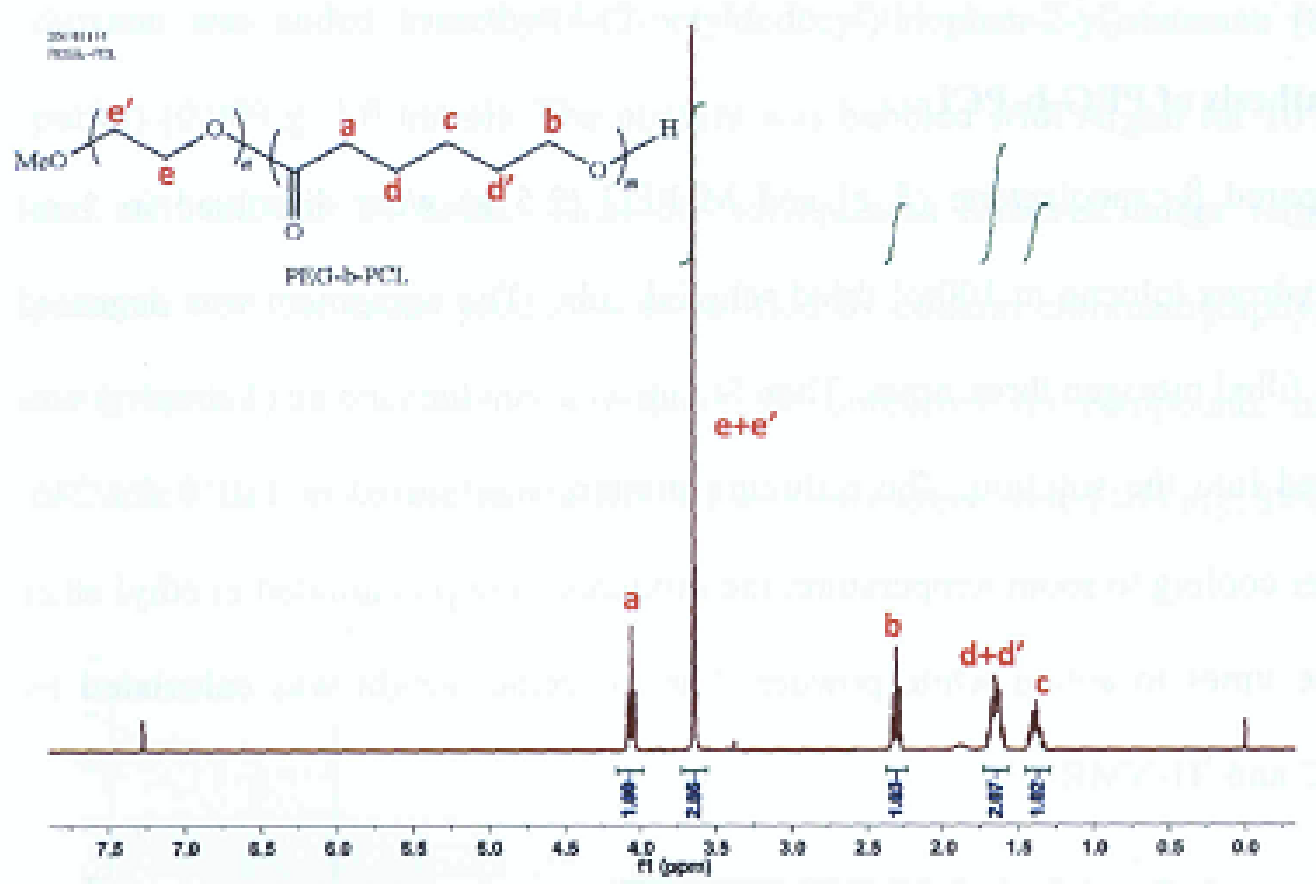

Figure 6-4. ${ }^{~} \mathrm{H}-\mathrm{NMR}\left(300 \mathrm{MHz}, \mathrm{CDCl}_{3}\right)$ spectrum of PEG-b-PCL.

\section{General procedure for the synthesis of the nanoparticles:}

Method A: A mixture of $14 \mathrm{mg}$ of BBT-2FT and $83 \mathrm{mg}$ of PEG-PCL was completely dissolved in $1 \mathrm{~mL}$ of THF overnight. Then $10 \mathrm{~mL}$ of deionized-water was quickly injected into the mixture under vigorous stirring at room temperature. After being stirred for $5 \mathrm{~min}$, the dispersion was dialyzed against deionized-water by $4 \mathrm{KDa}$ dialysis membranes for $48 \mathrm{~h}$ to remove THF. The NPs were separated by centrifugation at $10000 \mathrm{rpm}$ for $5 \mathrm{~min}$ and then redispersed in deionized-water before characterization and cell study. The concentrations used for following measurements and cell study are calculated based on the amount of BBT-2FT used in NP preparation.

Method B: A mixture of $14 \mathrm{mg}$ of BBT-2FT and $83 \mathrm{mg}$ of PEG-PCL was 
completely dissolved in $1 \mathrm{~mL}$ of THF overnight. Then $10 \mathrm{~mL}$ of deionized-water was quickly injected into the mixture under vigorous stirring at room temperature. The mixture was continuously stirred for $48 \mathrm{~h}$ in a cap-open vial at room temperature to remove THF.

\section{General materials characterization:}

Transmission electron microscope (TEM) measurements were carried out on a TEM Carl Zeiss Libra 120 Plus operating at an acceleration voltage of $120 \mathrm{kV}$. UV-vis transmission spectrum was recorded on a Varian Cary 4000 UV-Vis spectrophotometer. ${ }^{1} \mathrm{H}$ NMR spectra and ${ }^{13} \mathrm{C}$ NMR spectra were recorded on a Varian Inova-300 Instrument (300 MHz). MADLI-TOF MS spectra were carried on MALDI TOF/TOF ABI 4800.

\section{Evaluation of photothermal effect and photostability}

The stock dispersion of BBT-2FT NPs was diluted to 100,50 and $25 \mu \mathrm{g} / \mathrm{mL}$, respectively. At each concentration, a total of $2 m L$ dispersion was used in the evaluation of its photothermal effect under irradiation of a 808-nm laser with a power density of $1.77 \mathrm{~W} / \mathrm{cm}^{2}$. Every sample was irradiated for $10 \mathrm{~min}$ and allowed to cool down to the room temperature for the next $10 \mathrm{~min}$, which was counted as one cycle. Water served as the reference group and control group. Temperature was recorded by thermal couple every 10 seconds. To test the stability of sample, we irradiated $2 \mathrm{~mL} \mathrm{BBT-2FT} \mathrm{NPs} \mathrm{dispersion} \mathrm{under} \mathrm{the}$ 808-nm NIR laser with a power density of $1.77 \mathrm{~W} / \mathrm{cm}^{2}$ for 6 cycles. 
Temperature was recorded by thermal couple every 10 seconds.

\section{In vitro experiment}

HeLa cells were purchased from American Type Culture Collection (Virginia, USA). HeLa cells were cultured in Dulbecco's Modified Eagle's medium (DMEM) supplemented with 10\% (vol/vol) fetal bovine serum (FBS) and 1\% penicillin /streptomycin mixture at $37^{\circ} \mathrm{C}$ with $5 \% \quad \mathrm{CO}_{2}$. Cultures were maintained by adding or replacing the medium every 3-4 days to maintain a cell density of $10^{6}-10^{7}$ cells per mL. Since HeLa cell lines adhered to the surface of the culture flask during each passaging, the adherent cells were removed by replacing the culture medium by $1 \times$ Trypsin (Life Technologies, CA, USA) and putting it back in the incubator for 5 mins. Culture medium was then added to neutralise trypsin and cell counting was performed to check the cell density.

In the photothermal study, a total of 1-mL cell suspension with a cell density of $\sim 1 \times 10^{6}$ cells $/ \mathrm{mL}$ was transferred to a well plate, where it was mixed with BBT-2FT NPs and incubated for 6 hours in the incubator. After 6 hours, the excess BBT-2FT NPs were washed three times with the culture medium. To evaluate cell death, co-staining dye calcein AM/PI (Sigma-Aldrich, Missouri, USA) was added to the cell sample and was incubated for 15 minutes at $37^{\circ} \mathrm{C}$ with $5 \% \mathrm{CO}_{2}$, before the observation under a fluorescence microscope. Dead cells were stained by prodium iodide to red fluorescence, and live cells were stained by calcein AM to green fluorescence.

In vitro cytotoxicity. PrestoBlue assay was used to evaluate the effect of different concentrations of BBT-2FT NPs on cell viability in HeLa cells. HeLa 
cells were seeded on a 96-well plate (10000 cells per well) and cultured at $37{ }^{\circ} \mathrm{C}$ with $5 \% \mathrm{CO}_{2}$. After $12 \mathrm{~h}$ for attachment, BBT-2FT NPs at four indicated concentrations were added to the medium. After incubation for $24 \mathrm{~h}$, the culture medium was removed and cells were washed three times by $1 \times$ PBS to remove excess NPs. Then, PrestoBlue reagent diluted by DMEM were added to each wells and incubated at $37{ }^{\circ} \mathrm{C}$ with $5 \% \mathrm{CO}_{2}$. At the same time, PrestoBlue reagents diluted by DMEM were also added to blank wells without cells as control. After $1 \mathrm{~h}$ incubation, the absorbance at $571 \mathrm{~nm}$ (reference wavelength is $600 \mathrm{~nm}$ ) was detected by Plate Reader (Tecan Infinite M200 series Pro, Tecan Asia, Singapore). Percentage reduction of PrestoBlue reagent of each sample was calculated according to the formula provided in manufacturer's protocol.

\subsection{Results and discussion}

\subsubsection{The preparation and characterization of BBT-2FT NPs}

Scheme 6-2 shows the molecular structure of BBT-2FT and a schematic presentation of the NPs formed through a nanoprecipitation process. Narrow-band gap molecule BBT-2FT was synthesized following a procedure described in previous section, which was also confirmed by

${ }^{1} \mathrm{H}-\mathrm{NMR}$ (Figure. 6-2), ${ }^{13} \mathrm{C}-\mathrm{NMR}$, and MALDI-TOF mass spectroscopy (Figure. 6-1). BBT-2FT showed two absorption peaks in an organic solvent such as THF, one around at $450 \mathrm{~nm}$ with small shoulder and the 
other at $880 \mathrm{~nm}$, with an onset around $1300 \mathrm{~nm}$. The molar absorption coefficient of BBT-2FT in toluene at $808 \mathrm{~nm}$ was calculated to be (Figure 6-5)

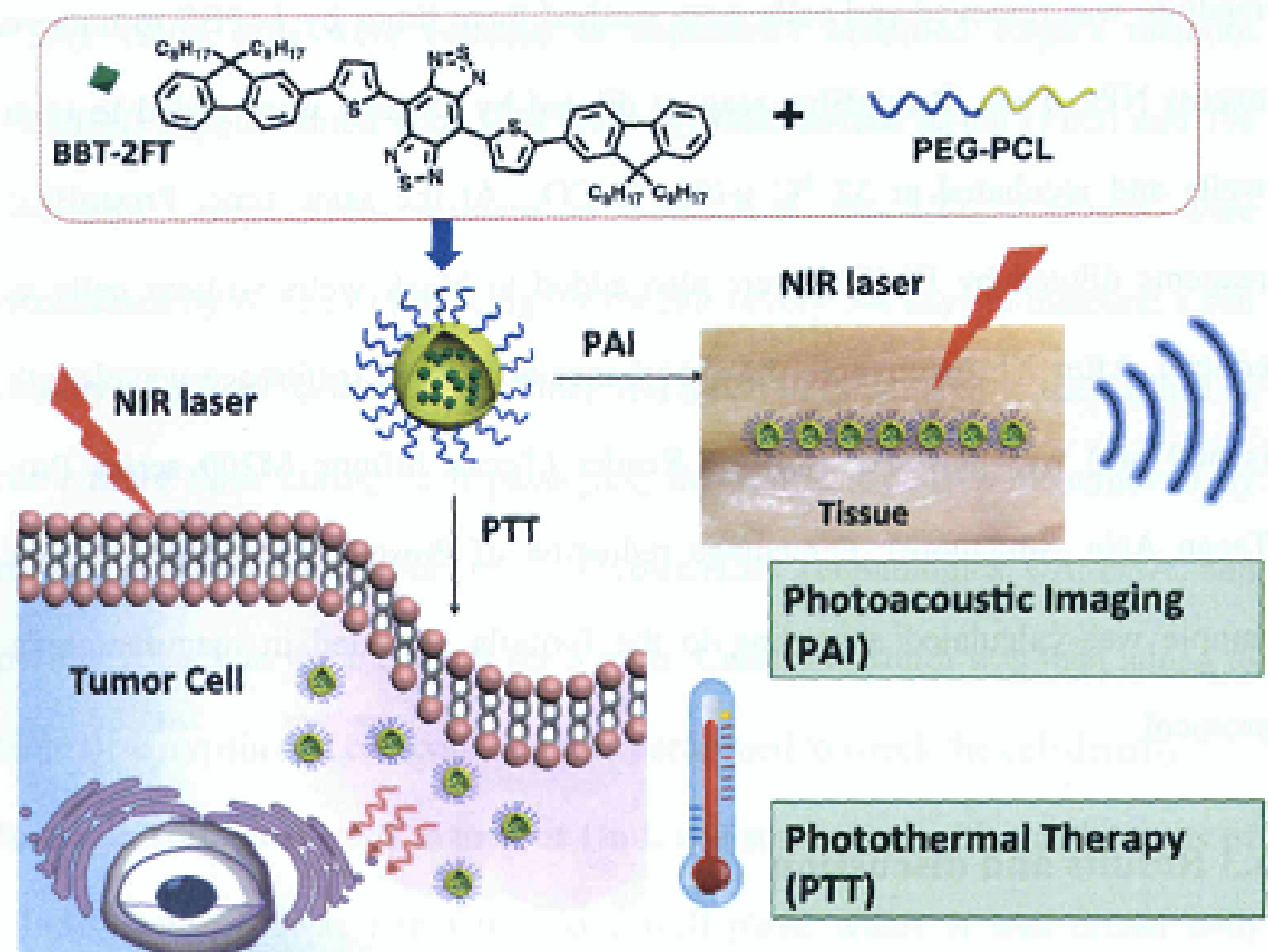

Scheme 6-2. Schematic illustration of the preparation of BBT-2FT nanoparticles and their applications in NIR photothermal therapy and photoacoustic imaging.

\section{Calculation of photothermal conversion efficiency}

The photothermal conversion efficiency of BBT-2FT NPs was determined according to previous method. ${ }^{64,65}$ The $\eta$ value was calculated as follow equation:

$$
\frac{h A \Delta T_{\max }-Q_{s}}{I \cdot\left(1-10^{-A_{\lambda}}\right)}
$$


where $\mathbf{h}$ is the heat transfer coefficient, $\mathbf{A}$ is the surface area of the container, $\Delta T_{\max }$ is the temperature change of the BBT-2FT solution at the maximum temperature, I is the laser power, $\mathbf{A}_{\boldsymbol{\lambda}}$ is the absorbance of BBT-2FT at $808 \mathrm{~nm}$, $\mathbf{Q}_{\mathbf{s}}$ is the heat change of the solvent, and $\boldsymbol{\eta}$ is the photothermal conversion efficiency. The unknown $\mathbf{h} \mathbf{A}$ value was determined by the linear data of time versus $-\ln \theta$ curve (Figure 6-6).

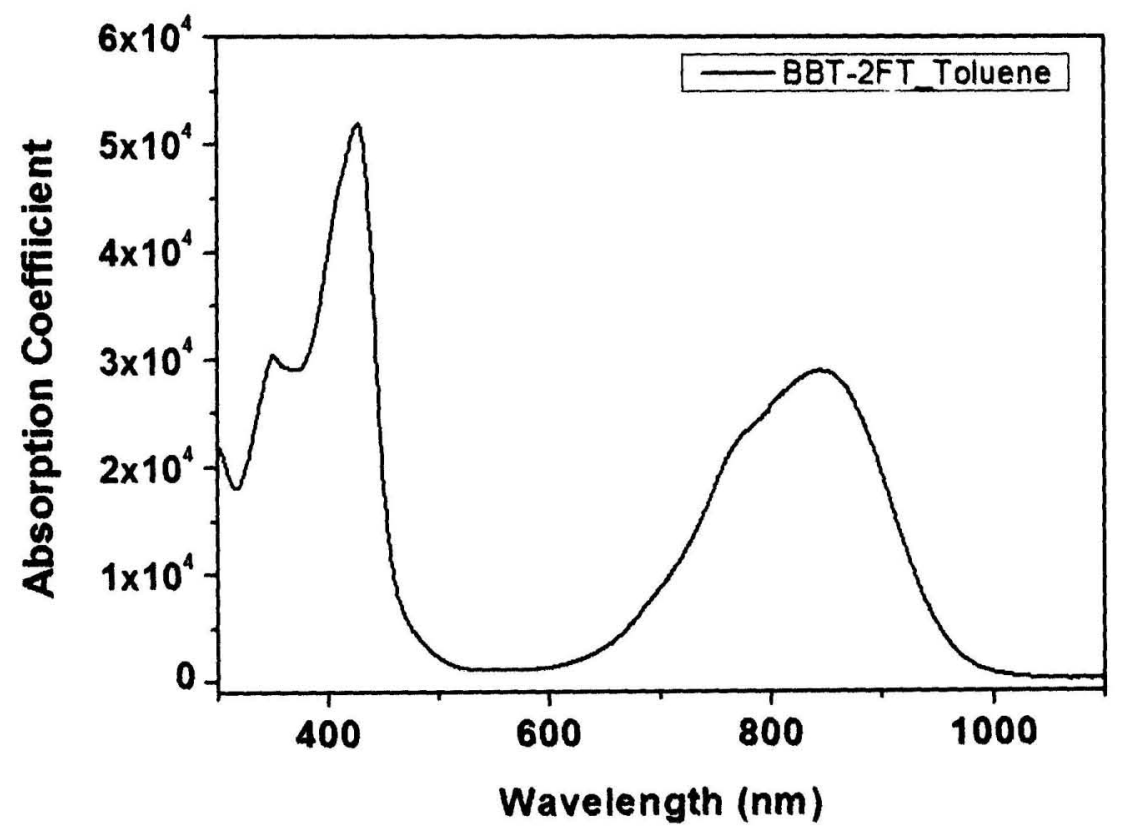

Figure 6-5. Molar absorption coefficient of BBT-2FT in toluene. 


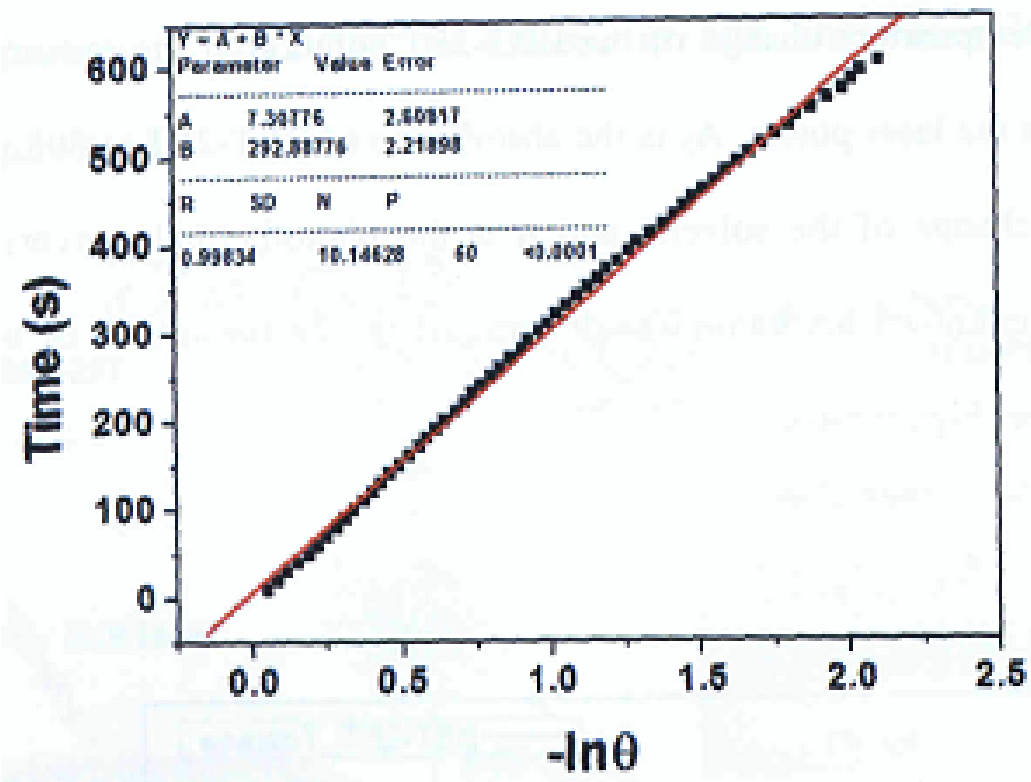

Figure 6-6. Linear time versus $-\ln \theta$ abtained from cooling period.

Herein, I chose an amphiphilic block polymer PEG- $b$-PCL instead of Pluoronic F127 as the stabilizer for the preparation of BBT-2FT colloidal NPs, as some agglomeration of Pluoronic F127 micelles loaded with BBT-EHT was observed under NIR laser light irradiation. ${ }^{60}$ Such intermicellar agglomeration can be attributed the relatively low lower critical solution temperature (LCST) of Pluronic F127 compared to that of PEG- $b$-PCL. As a result, Pluronic F127 forms gels in water over a much broader temperature range than PEG- $b$-PCL- $b$-PEG. ${ }^{66,67}$ These results suggest that PEG- $b$-PCL micelles have better photothermal stability than Pluronic F127 micelles, which can benefit their applications in bioiamging and photothermal therapy. PEG- $b$-PCL was synthesized via 
ring-opening polymerization of $\beta$-caprolactone using monomethyl-PEG $\mathrm{F}_{5 \mathrm{k}}$ as the initiator. The number average molecular weight $\left(M_{n}\right)$ calculated by GPC was 11000 , with the ratio of hydrophylic PEG chain to hydrophobic PCL chain is $1: 1$, which is consistent with the result calculated by ${ }^{1} \mathrm{H}-\mathrm{NMR}$. Details about the synthesis and characterization of PEG- $b$-PCL are presented in previous section.

Two methods were tested to prepare the colloidal NPs of BBT-2FT in presence of PEG- $b$-PCL (critical micelle concentration $=5 \mathrm{mg} \mathrm{mL}^{-1}$ in water) ${ }^{68}$ that not only ensures good biocompatibility, but also enhances colloidal stability of BBT-2FT NPs in water. The experimental details about the preparation of the NPs are described in Supporting Information. Briefly, in Method A, the mixture of BBT-2FT and PEG- $b$-PCL in THF/water (1/10, by volume) was subjected to dialysis against water to remove THF. In Method $\mathrm{B}, \mathrm{THF}$ in the mixture was removed by evaporation in air under vigorous stirring at room temperature. In both cases, the self-assembly was driven by the hydrophobic interaction between the PCL and BBT-2FT which collapsed to form the core of the NPs, while the hydrophilic PEG forming the shell of the NPs provided colloidal stability. 
(a)
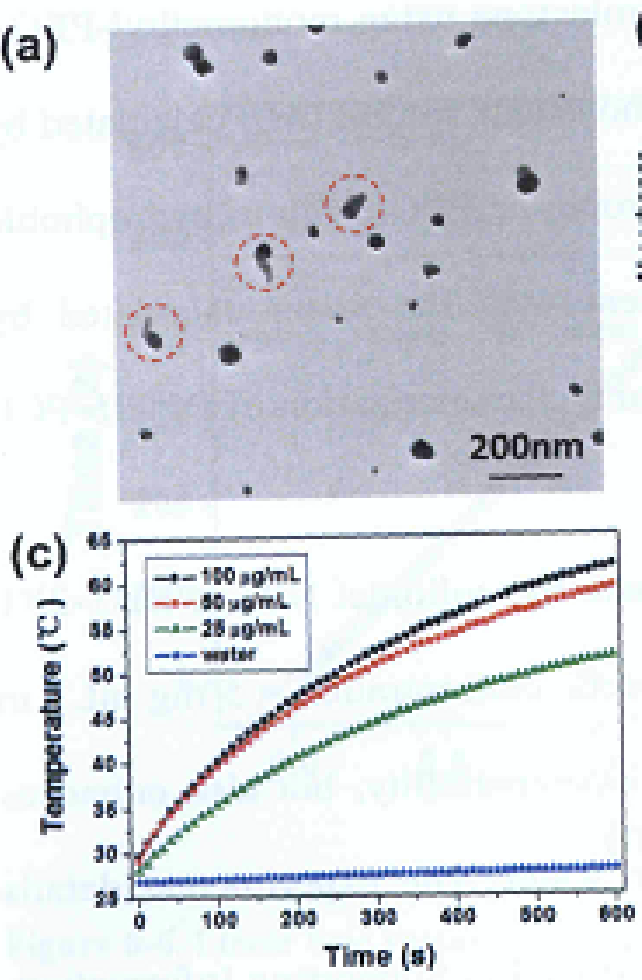

(b)
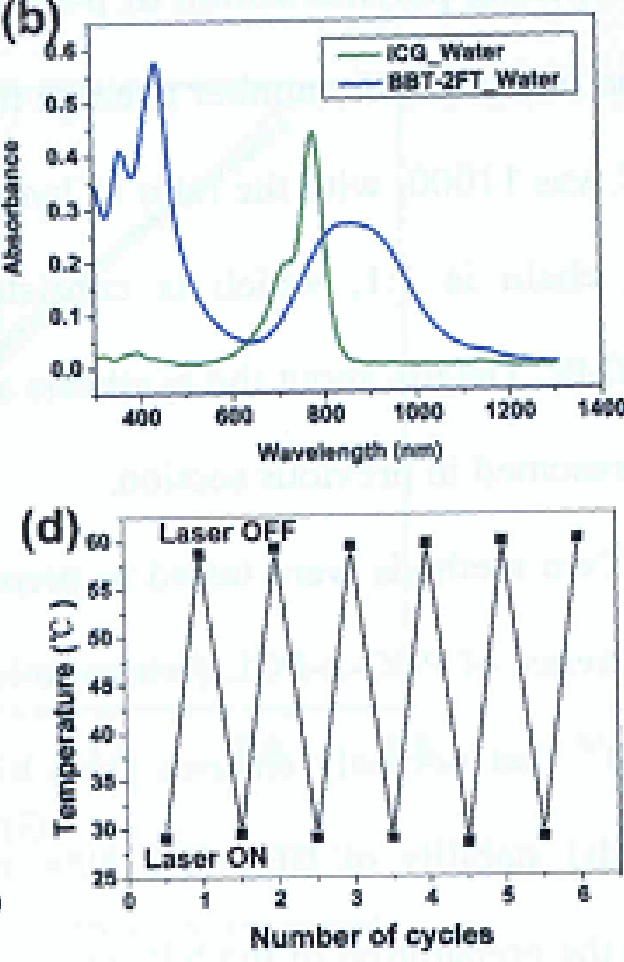

Figure 6-7. (a) TEM image of BBT-2FT NPs from dispersion of $50 \mu \mathrm{g} \mathrm{mL-1.} \mathrm{(b)}$ UV-Vis-NIR spectra of BBT-2FT NPs dispersed in water and ICG water solution. (c) Temperature change plots of different concentrations of BBT-2FT NPs upon irradiation by an $808 \mathrm{~nm}$ laser with a power density if $1.77 \mathrm{~W} / \mathrm{cm}^{2}$ for 10 min. (d) Temperature elevation of BBT-2FT NPs over six laser ON/OFF cycles of NIR laser irradiation. All of the NPs presented in this figure were prepared through Method A. All of the concentrations of the BBT-2FT NPs are calculated based on the mass of BBT-2FT without inclusion of the stabilizer.

PEG- $b$-PCL micelles before and after being loaded with BBT-2FT were characterized by both transmission electron microscopy (TEM) and dynamic light scattering (DLS). A representative TEM image of the BBT-2FT-loaded PEG- $b$-PCL micelles prepared by Method A is shown 
in Figure 6-7 a. Most of the NPs appear spherical with different sizes, while a minor population of tadpole-like particles (labeled by circles in Figure 6-7 a) was also observed. The average diameter of these NPs measured by TEM is $46 \pm 11 \mathrm{~nm}$, which is smaller than that $(68 \mathrm{~nm})$ measured by DLS in the hydrated state. Both TEM and DLS results showed that the average size of the BBT-2FT-loaded PEG- $b$-PCL micelles was slightly larger than that of PEG- $b$-PCL blank micelles (Figure 6-8) prepared by the same method.
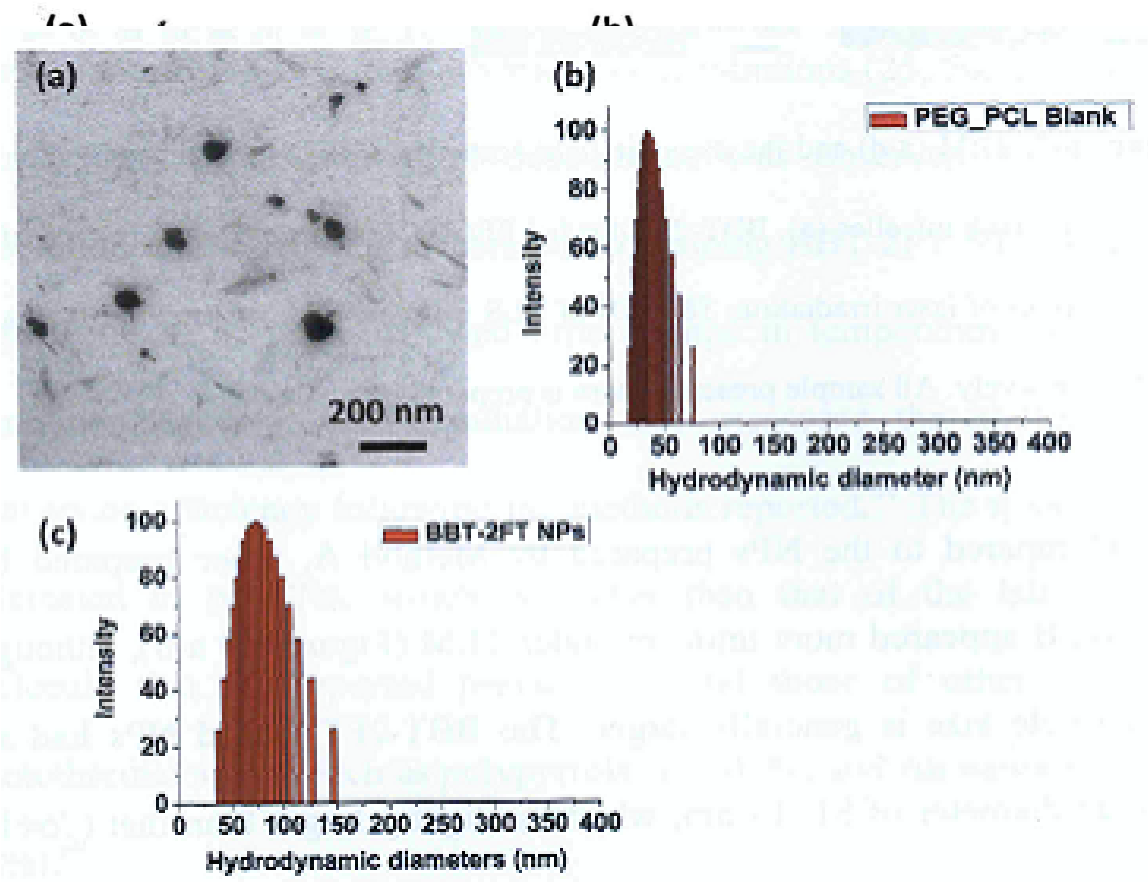

Figure 6-8. TEM image (a) and DLS result (b) of blank PEG- $b$-PCL micelles prepared by Method A, showing $42 \pm 14 \mathrm{~nm}$ and $40 \mathrm{~nm}$ (PDI: 0.27) respectively. Dynamic light scattering analysis (c) of BBT-2FT NPs, showing $68 \mathrm{~nm}$ (PDI: 0.28). 

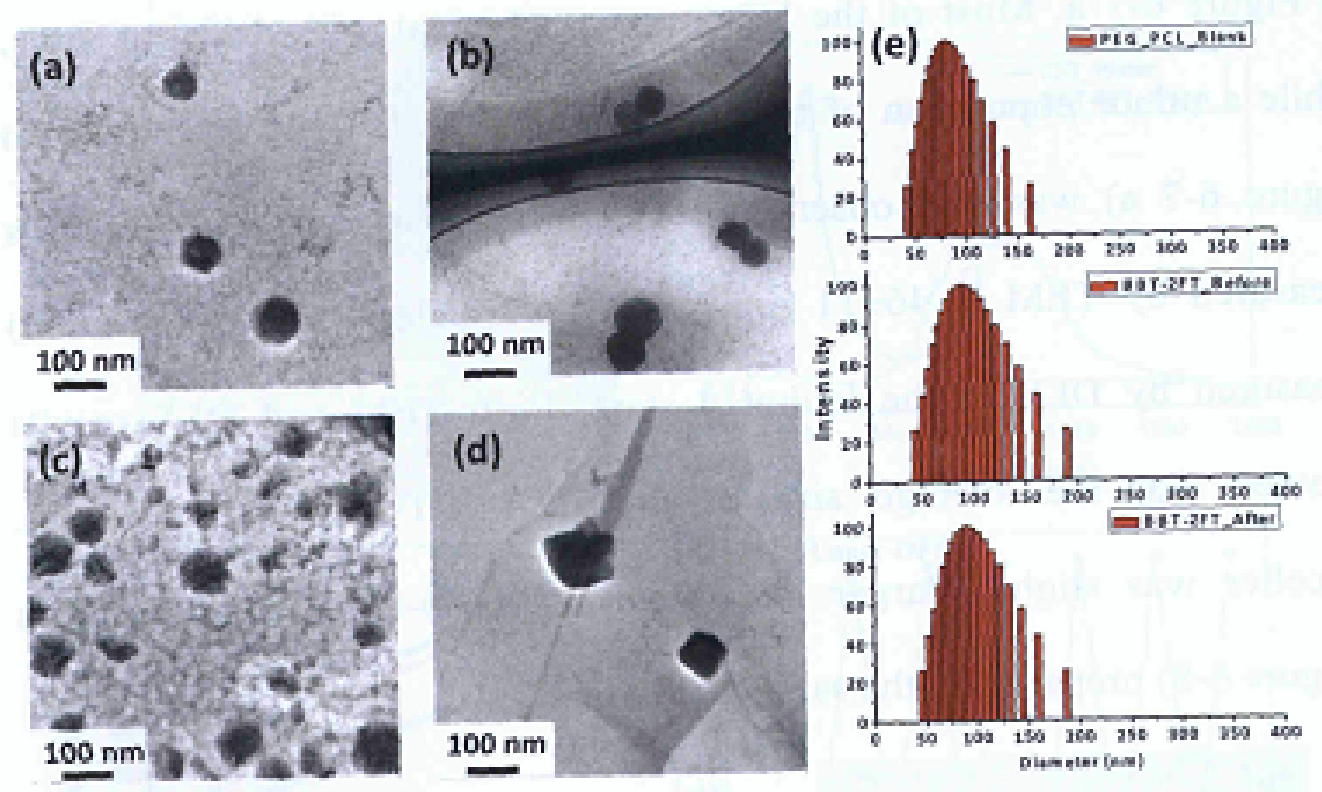

Figure 6-9. TEM (a-d) and the dynamic light scattering (DLS) (e) characterization of PEG-PCL blank micelles (a), BBT-2FT-loaded PEG-PCL micelles before (b) and after (c,d) 6 cycles of laser irradiation. The PDI of DLS from tup to down are $0.26,0.29$ and 0.29 respectively. All sample presented here is prepared by method B.

Compared to the NPs prepared by Method A, those prepared by Method B appeared more uniform under TEM (Figure 6-9 a-b), although the particle size is generally larger. The BBT-2FT-loaded NPs had an average diameter of $81 \pm 13 \mathrm{~nm}$, which is slightly larger than that $(76 \pm 16$ $\mathrm{nm})$ of PEG- $b$-PCL blank micelles. The hydrodynamic diameter of the hydrated micelles in water measured by DLS also increased from 90 to $100 \mathrm{~nm}$ after being loaded with BBT-2FT. These micellar particles with suitable sizes are expected to target cancer tissues through enhanced permeation retention (EPR) effect and to prevent a blockage of blood 
vessels or being eliminated by the body's reticuloendothelial system (RES). Most of the experiments below involved BBT-2FT NPs prepared through Method A unless noted specifically.

BBT-2FT shows a strong NIR absorption peak at $880 \mathrm{~nm}$, which is broader compared to that of commercial ICG molecules. The photothermal effect induced by NIR laser illumination at $808 \mathrm{~nm}$ with a power density of $1.77 \mathrm{~W} \mathrm{~cm}^{-2}$ for $10 \mathrm{~min}$ in the presence of BBT-2FT NPs was investigated by monitoring the temperature of $1 \mathrm{~mL}$ aqueous dispersion of BBT-2FT NPs at various concentrations $(25,50$, and $100 \mu \mathrm{g}$ $\mathrm{mL}^{-1}$ ). Obvious concentration dependence was observed under laser irradiation of the aqueous dispersions containing BBT-2FT NPs, whereas pure water as a control showed little change in temperature under the same conditions of laser irradiation. We measured the photothermal conversion efficiency following the methods reported. ${ }^{69}$ The $\eta$ value was calculated to be $47 \%$, which is higher than that of the BBT small molecule that we reported previously ${ }^{60}$ and those of other reported photothermal agents such as polypyrrole $(\eta=40 \%)$ and Au nanorods $(\eta=$ $22 \%)^{69}$

To further investigate the photostability of BBT-2FT NPs, six cycles of laser ON/OFF with NIR laser were used. The continuous laser $(808 \mathrm{~nm}$, $1.77 \mathrm{~W} / \mathrm{cm}^{2}$ ) instead of high power pulse laser was used to test the photothermal effect, since the pulse laser used in photoacoustic system causes the temperature increasing too fast to record. We note that the 
continuous laser was also used in the cell experiments described later. Dispersion of BBT-2FT NPs $\left(50 \mu \mathrm{g} \mathrm{mL} L^{-1}\right)$ was irradiated with NIR laser for $10 \mathrm{~min}$ (Laser ON, Figure 6-7 d), followed by naturally cooling (without laser irradiation) to room temperature for $10 \mathrm{~min}$ (Laser OFF). This cycle was repeated six times in order to investigate the photostability of BBT-2FT NPs. The recorded temperature change indicated no significant photoinduced degradation of BBT-2FT NPs under the present experimental conditions (Figure. 6-7 d). Nevertheless, the TEM images (Figure 6-9 c-d) showed some morphological changes of the nanoparticles (prepared via Method B) after laser irradiation. For example, the overall size distribution of the micelles appeared broader $(87 \pm 19 \mathrm{~nm}$ by TEM) after 6 cycles of ON/OFF laser treatment, and some polyhedral nanoparticles were observed. Such changes might be caused by the photothermal annealing effect that induced the local crystallization of the PCL chains within the cores of the micelles. Nevertheless, the morphological and size changes observed under TEM could not be detected by dynamic light scattering (Figure 6-9 e), suggesting that the average size and colloidal stability of the nanoparticles were not significantly affected by the laser irradiation.

The promising photothermal conversion efficiency of BBT-2FT NPs prompted us to investigate the application of these NPs for photoacoustic imaging as well as photothermal therapy, as discussed below. 


\subsubsection{PAT imaging system}

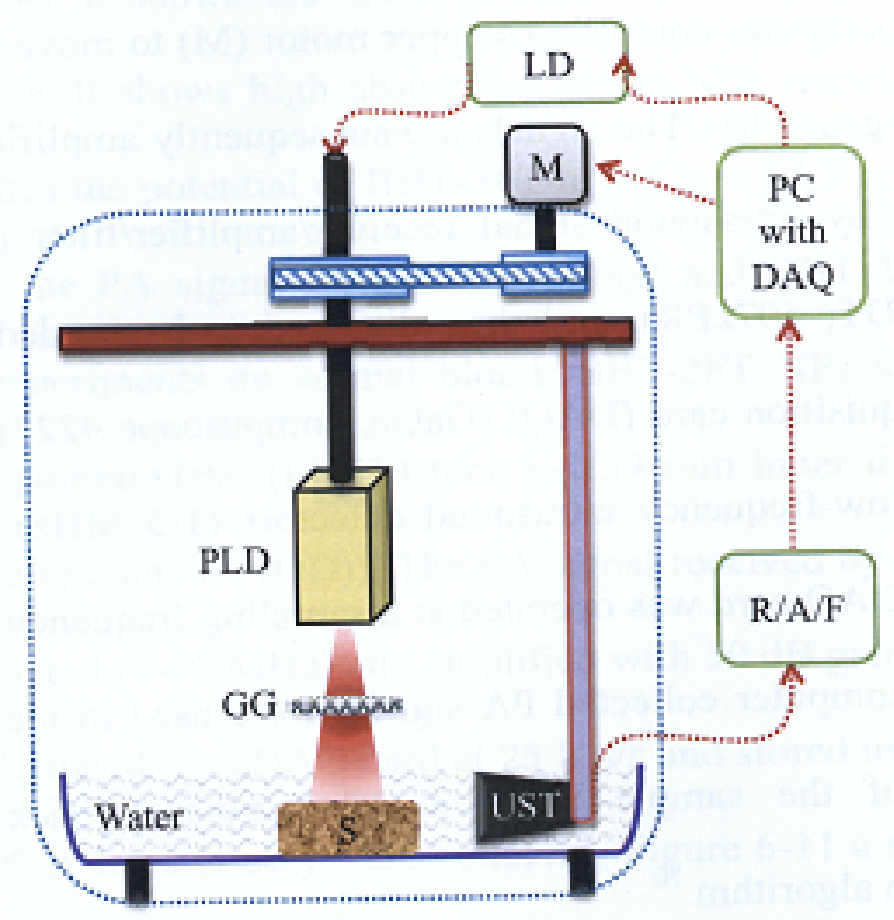

Figure 6-10. Schematic diagram of the PLD-PAT imaging system. PLD: Pulsed laser diode, GG: Ground glass, S: Sample, M: Motor, LD: Laser driver, R/A/F: Receiver, amplifier and filter unit, DAQ: Data acquisition card, UST: Ultrasound transducer.

The PAT imaging system used in the current study is depicted in Figure 6-10. The pulsed laser diode (PLD) (Quantel, France) provides $\sim 136 \mathrm{~ns}$ pulses at a wavelength of $\sim 803 \mathrm{~nm}$ and pulse energy of $\sim 1.4 \mathrm{~mJ}$ at maximum $7 \mathrm{kHz}$ repetition rate. A ground glass (GG) is used to make the laser beam more homogeneous. The sample and the transducer were immersed in water for coupling of PA signal to the ultrasound transducer (UST). The photoacoustic (PA) signal generated by the sample was 
received by a non-focus transducer (V323-SU/2.25 MHz, Olympus NDT) with $13 \mathrm{~mm}$ active area and $\sim 70 \%$ nominal bandwidth. The UST was driven by a computer-controlled stepper motor (M) to move continuously in a circular geometry. The signals are subsequently amplified, and band pass filtered by ultrasound signal receiver/amplifier/filter $(\mathrm{R} / \mathrm{A} / \mathrm{F})$ unit (Olympus-NDT, 5072PR), and then digitized and recorded by the PC with data acquisition card (DAQ) (GaGe, compuscope 4227) installed in it. Usually, low-frequency ultrasound detectors $(1-5 \mathrm{MHz})$ are used in PAT, so the DAQ card was operated at a sampling frequency of $25 \mathrm{Ms} / \mathrm{s}$. Finally, the computer collected PA signals were used to reconstruct the PA image of the sample using a delay-and-sum back projection reconstruction algorithm..$^{70}$

I note that local temperature rise in the order of few millidegrees can produce photoacoustic signal strong enough for imaging. For instance, one millidegree of temperature rise produces $8 \mathrm{mbar}$ of pressure rise. The ultrasound detectors used for photoacoustic imaging are sensitive enough to record these pressure waves. Moreover, the imaging time is quite short. It has been shown that the photoacoustic cross sectional imaging can be performed as low as in 3 second imaging time. ${ }^{70}$ If one uses photoacoustic imaging system based on an ultrasound array transducer, one can obtain photoacoustic imaging in a single laser pulse. Therefore, the photoacoustic imaging itself will not cause any significant temperature rise to initiate the photothermal therapy process. 


\subsubsection{Photoacoustic signals from blood/BBT-2FT samples}

Figure 6-7 $\mathrm{b}$ shows the UV-VIS-NIR extinction spectrum of the BBT-2FT NPs. It shows high absorption in the NIR wavelength region which indicates the potential of BBT-2FT to act as a PAT contrast agent. To compare the PA signal from animal blood and BBT-2FT NPs, we performed experiments on animal blood/ BBT-2FT NPs sample inside low density polyethylene (LDPE) tube $(\sim 0.59 \mathrm{~mm}$ inner diameter (ID), $\sim 0.78 \mathrm{~mm}$ outer diameter (OD)). The PA signal received by the UST was band pass filtered (1-10 MHz) and amplified with $50 \mathrm{~dB}$ gain. Finally, the signal was digitized by a DAQ card at $25 \mathrm{Ms} / \mathrm{s}$ and stored in computer. A total of 7,000 A-lines (1 sec) were collected. Figure 6-11 a shows the PA signals averaged 700 times from animal blood and BBT-2FT NPs (2 $\mathrm{mg} / \mathrm{mL}$ ). The signal from BBT-2FT NPs is $\sim 10$ times stronger than that from blood. Figure 6-11 b shows that the PA signal intensities increased linearly with the concentration of BBT-2FT NPs.

\subsubsection{Deep-tissue imaging experiment}

To check the feasibility of BBT-2FT NPs as a PAT contrast agent and determine their effective imaging depth at a wavelength of $803 \mathrm{~nm}$, we acquired PA signals of blood and BBT-2FT NPs embedded inside a chicken breast tissue. 
The LDPE tube filled with bloor or BBT-2FT NPs $(2 \mathrm{mg} / \mathrm{mL})$ was embedded in the chicken breast tissue. PA signals were collected when the tube was placed at $1,2,3$, or $4 \mathrm{~cm}$ deep from the laser illuminated tissue surface. The incident laser energy density on the tissue surface area is $\sim 0.28 \mathrm{~mJ} / \mathrm{cm}^{2}$, which is much less than the "maximum permissible exposure (MPE)" of $32 \mathrm{~mJ} / \mathrm{cm}^{2}$ at $803 \mathrm{~nm}$. Figures 6-11 $\mathrm{c}$ and d show the PA signals collected from blood and BBT-2FT $(2 \mathrm{mg} / \mathrm{mL})$ at different depths (D) by the $2.25 \mathrm{MHz}$ UST. In our current experiments, blood and BBT-2FT NPs were successfully detected in chicken breast tissue at depth of $\sim 2.0 \mathrm{~cm}$, and $\sim 4.0 \mathrm{~cm}$ respectively. The imaging depth can be further increased by using a higher power pulsed laser (for example optical parametric oscillator (OPO) laser pumped by $\mathrm{Nd}$ :YAG laser producing more than 70 times stronger energy per pulse).

Deep-tissue imaging experiments were carried out on the sample shown in Figure 6-12 a, which is made of two LDPE tubes $(\sim 0.59 \mathrm{~mm} \mathrm{ID,}$ $\sim 0.78 \mathrm{~mm}$ OD, $\sim 8 \mathrm{~mm}$ long), one filled with blood and other filled with BBT-2FT NPs (2 mg/mL). The two LDPE tubes were placed on a chicken breast tissue as shown in Figure 6-12 a. For imaging they were covered by tissues of various thicknesses as shown in Figure 6-12 b. The tissue cross-section containing the LDPE tubes was imaged when tissue slices were sequentially placed to make the tubes $1 \mathrm{~cm}$, and $2 \mathrm{~cm}$ deep from laser-illuminated tissue surface. Figure 6-12 $\mathrm{c}$ and d show the PAT images acquired at $1 \mathrm{~cm}$, and $2 \mathrm{~cm}$ depth, respectively. The SNR values 
of blood, BBT-2FT NPs measured from Figure 6-12 $\mathrm{c}$ are $\sim 23, \sim 35$ and that measured from Figure 6-12 d are $\sim 9, \sim 14$, respectively. Both the tubes were clearly visible at $2 \mathrm{~cm}$ under the chicken breast tissues. Our results indicate that BBT-2FT NPs are promising contrast agents for PAT with good PA signal enhancement and image contrast in biological tissues.
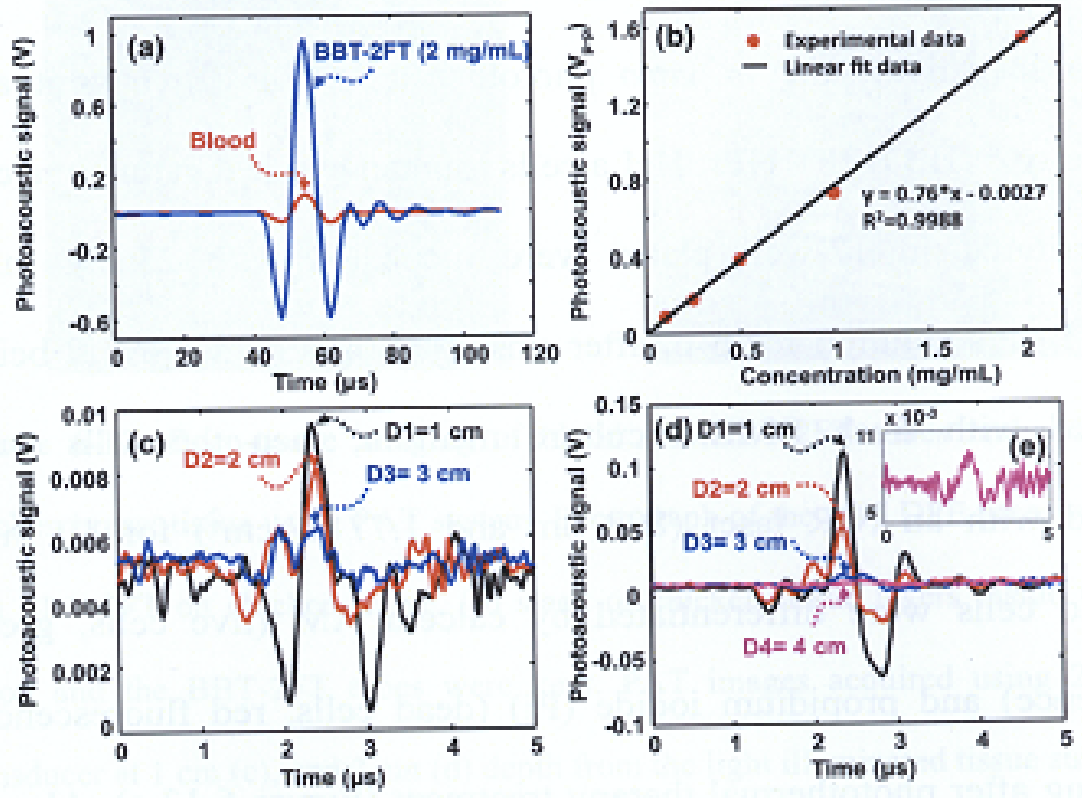

(f)

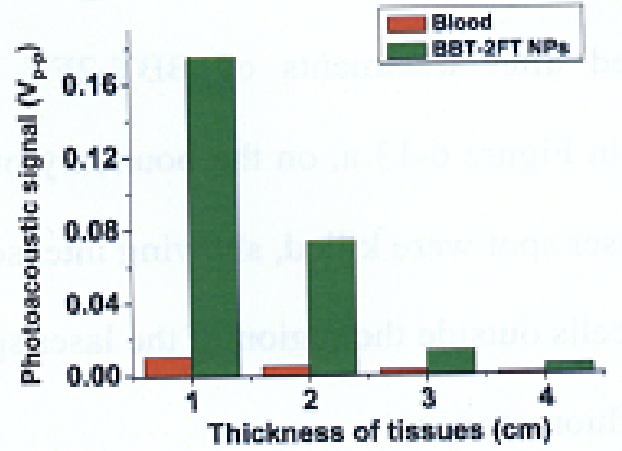

Figure 6-11. Photoacoustic (PA) signal of BBT-2FT compared with blood. (a) PA signals of blood and BBT-2FT NPs ( $2 \mathrm{mg} / \mathrm{mL})$ received by $2.25 \mathrm{MHz}$ UST, (b) PA 
signal as a function of concentration of BBT-2FT NPs. PA signals generated from blood (c) and BBT-2FT NPs (d) in a LDPE tube embedded inside a chicken breast tissue at difference depths, (e) Zoom in version of the PA signal at D4 $=4 \mathrm{~cm}$. (f) The PA signal of blood and BBT-2FT NPs as a function of penetration depth in chicken tissues.

\subsubsection{Photothermal therapy in vitro experiments}

We next investigate the in vitro photothermal therapy treatment and cytotoxicity of BBT-2FT NPs. HeLa cells (human cervical carcinoma cell lines) cultured in 12 -well plates were incubated with $25 \mu \mathrm{g} \mathrm{mL^{-1 }}$ BBT-2FT NPs solution for $6 \mathrm{~h}$, after rinsing with PBS twice and being resupplied with fresh DMEM culture medium, then the cells were irradiatcd with an NIR laser $\left(808 \mathrm{~nm}\right.$ and $\left.1.77 \mathrm{~W} / \mathrm{cm}^{2}\right)$ for $10 \mathrm{~min}$. Live/dead cells were differentiated by calcein AM (live cells, green fluorescence) and propidium iodide (PI) (dead cells, red fluorescence) co-staining after photothermal therapy treatment (Figure 6-13 a). Almost $90 \%$ cells were killed after treatments of BBT-2FT NPs and laser irradiation. As shown in Figure 6-13 a, on the boundary of the laser spot, only cells within the laser spot were killed, showing intense homogeneous red fluorescence. The cells outside the region of the laser spot stayed alive, showing strong green fluorescence. 

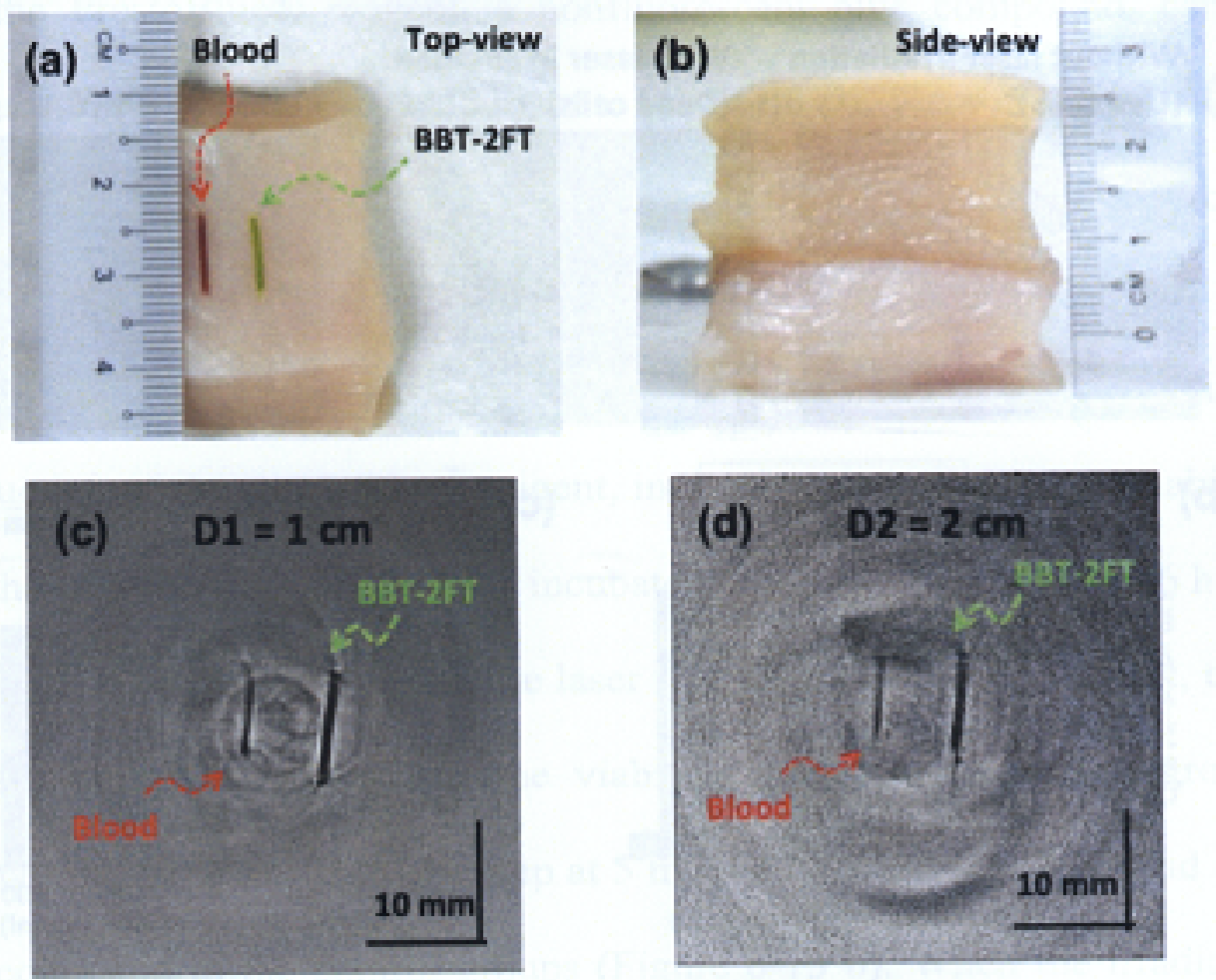

Figure 6-12. Deep-tissue imaging of blood and BBT-2FT tubes embedded inside chicken breast tissue using PAT system. Photograph of the (a) LDPE tubes with blood and BBT-2FT on chicken tissue, (b) stack of chicken tissue layers inside which the blood and the BBT-2FT tubes were kept. PAT images acquired using $2.25 \mathrm{MHz}$ transducer at $1 \mathrm{~cm}(\mathrm{c})$, and $2 \mathrm{~cm}(\mathrm{~d})$ depth from the light illuminated tissue surface. 

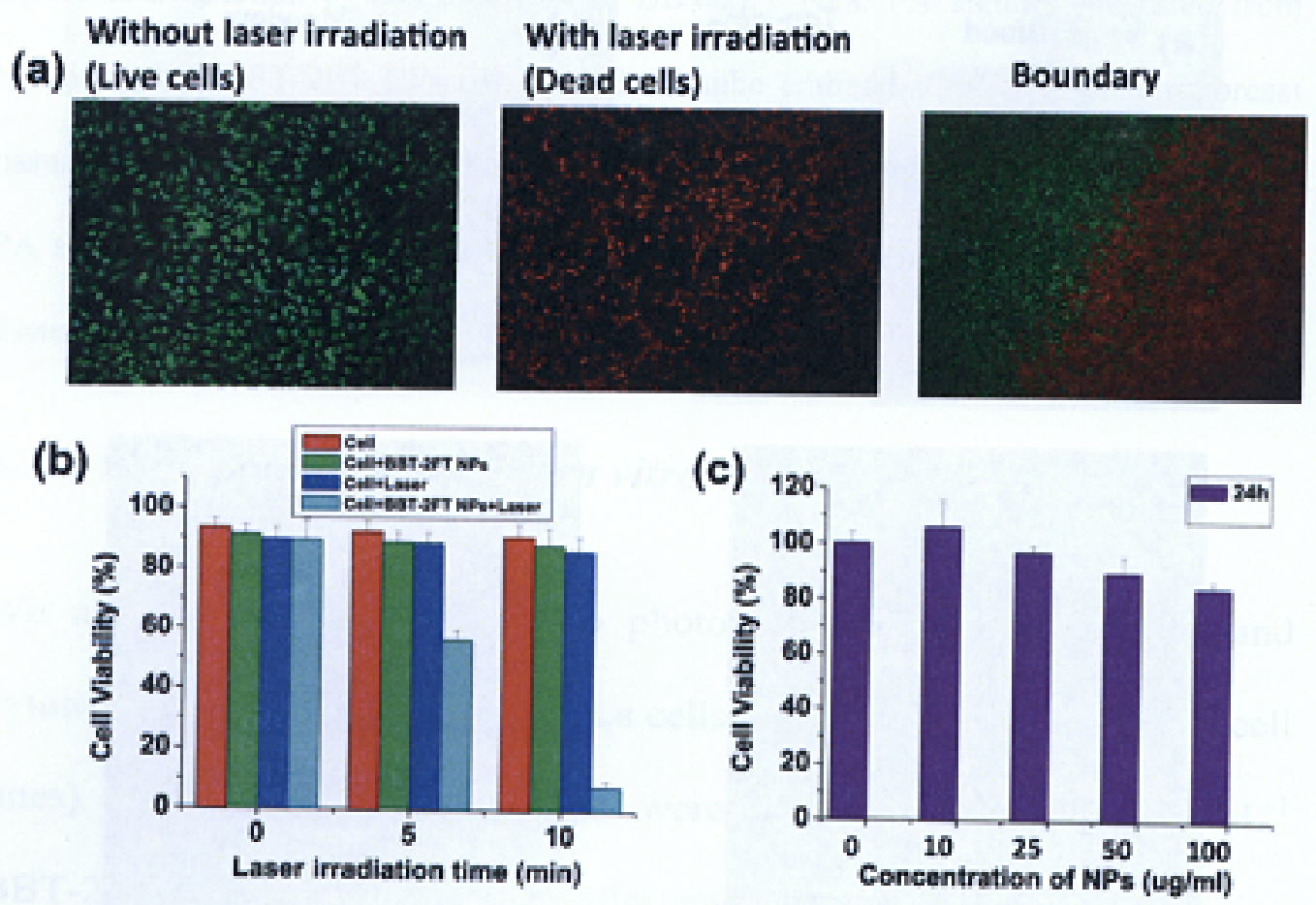

Figure 6-13. In vitro cell experiments. (a) Fluorescence images of calcein AM/PI co-stained HeLa cells after incubation for $6 \mathrm{~h}$ with BBT-2FT NPs $\left(25 \mu \mathrm{g} \mathrm{mL} \mathrm{L}^{-1}\right)$ after being irradiated by laser $\left(808 \mathrm{~nm}\right.$ and $\left.1.77 \mathrm{~W} / \mathrm{cm}^{2}\right)$ for $10 \mathrm{~min}$. (b) Viabilities of $\mathrm{HeLa}$ cells after PTT at different laser irradiation time. Cell viability was normalized to the control group without any treatment. Error bars are based on the standard deviations of five parallel samples. (c) Viability of HeLa cells after being incubated with various concentrations of BBT-2FT NPs for $24 \mathrm{~h}$ tested by PrestoBlue ${ }^{\mathbb{B}}$ reagent without laser irradiation.

To evaluate the photothermal cytotoxicity of BBT-2FT NPs in a more quantitative way, HeLa cell lines were irradiated under laser for

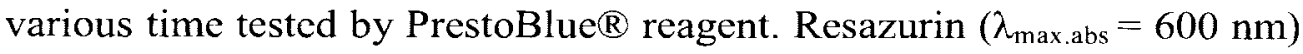


in the PrestoBlue ${ }^{\circledR}$ reagent, a nonfluorescent blue compound, can be reduced in live cells by metabolism to resorufin $\left(\lambda_{\max . a b s}=571 \mathrm{~nm}\right)$, which is red in color and highly fluorescent. Since the number of metabolically active cells proportionally correlates with the reduction level, the absorbance readings can be converted and expressed as the percentage reduction of the PrestoBlue ${ }^{\circledR}$ reagent, indicating the relative cell viability. In the experimental group, cells incubated with BBT-2FT NPs for $6 \mathrm{~h}$ in a 96-well plate was exposed to the laser for $5 \mathrm{~min}$. At this time point, there was no apparent change in the viability of the three control groups. However, the experimental group at 5 min showed almost $50 \%$ dead cells as compared to the control groups (Figure 6-13 b). When the irradiation time was prolonged to $10 \mathrm{~min}$, there were nearly no viable cells, resulting in close to $100 \%$ cell death in the experimental group (Figure 6-13 b).

To examine the biocompatibility of BBT-2FT NPs, HeLa cells were incubated with gradient concentration of NPs dispersion for $24 \mathrm{~h}$ whithout laser irradiation. Figure 6-13 c shows the dose-dependence the cytotoxicity of NPs against HeLa cells. One can see that BBT-2FT NPs show minimal toxicity to HeLa cells without NIR laser irradiation. These results suggest that BBT-2FT NPs can serve as a promising photothermal agent for cancer therapy. 


\subsection{Conclusions}

In this chapter, I presented a dual-modal photoacoustic imaging and photothermal therapeutic agent based on BBT-2FT NPs with strong absorption in the NIR region. These NPs exhibit good colloidal stability, obviously stronger photoacoustic signal than blood, higher photothermal conversion efficiency, and excellent photostability. Almost 10 times stronger photoacoustic intensity than blood was detected, and $4 \mathrm{~cm}$ tissue penetration depth makes it viable for in vivo applications. Moreover, significant death of HeLa cells was observed due to the hyperthermal effect. These results demonstrate that the BBT-based NPs are promising theranostic agents for cancer imaging and therapy. Further application of these dual functional agents in animals and clinical trial is under investigation, which, if successful, will enable the "see and treat" strategy using a single platform.

\subsection{References}

1. J. Funkhouser, Curr. Drug Discovery, 2002, 2, 17-19.

2. S. S. Kelkar and T. M. Reineke, Bioconjug. Chem., 2011, 22, 1879-1903.

3. T. Krasia-Christoforou and T. K. Georgiou, J. Mater. Chem. B, 2013, 1, 3002-3025.

4. A. Ito, M. Shinkai, H. Honda and T. Kobayashi, J. Biosci. Bioeng., 2005, $100,1-11$. 
5. J.-M. Hsieh, M.-L. Ho, P.-W. Wu, P.-T. Chou, T.-T. Tsai and Y. Chi, Chem. Commun., 2006, 615-617.

6. F. L. Primo, M. M. Rodrigues, A. R. Simioni, Z. G. Lacava, P. C. Morais and A. C. Tedesco, J. Nanosci. Nanotechnol., 2008, 8, 5873-5877.

7. Z. Liu, K. Chen, C. Davis, S. Sherlock, Q. Cao, X. Chen and H. Dai, Cancer Res., 2008, 68, 6652-6660.

8. J. Yuan, W. Guo, X. Yang and E. Wang, Anal. Chem., 2009, 81, 362-368.

9. Z. Liu, A. C. Fan, K. Rakhra, S. Sherlock, A. Goodwin, X. Chen, Q. Yang, D. W. Felsher and H. Dai, Angew. Chem. Int. Ed. , 2009, 48, 7668-7672.

10. Y. F. Tan, P. Chandrasekharan, D. Maity, C. X. Yong, K. H. Chuang, Y. Zhao, S. Wang, J. Ding and S. S. Feng, Biomaterials, 2011, 32, 2969-2978.

11. C. Li and L. V. Wang, Phys. Med. Biol., 2009, 54, R59-97.

12. L. V. Wang, Nature Photon., 2009, 3, 503-509.

13. P. Beard, Interface focus, $2011,1,602-631$.

14. L. V. Wang and S. Hu, Science, 2012, 335, 1458-1462.

15. X. Wang, X. Xie, G. Ku, L. V. Wang and G. Stoica, J. Biomed. Opt., 2006, 11, 024015

16. L. V. Wang, Med. Phys., 2008, 35, 5758-5767.

17. E. Z. Zhang, J. G. Laufer, R. B. Pedley and P. C. Beard, Phys. Med. Biol., $2009,54,1035-1046$.

18. M. Pramanik and L. V. Wang, J. Biomed. Opt., 2009, 14, 054024.

19. T. N. Erpelding, C. Kim, M. Pramanik, L. Jankovic, K. Maslov, Z. Guo, J. A. Margenthaler, M. D. Pashley and L. V. Wang, Radiology, 2010, 256, 
102-110.

20. S. Hu and L. V. Wang, J. Biomed. Opt., 2010, 15, 011101.

21. W. F. Xia, D. Piras, M. K. A. Singh, J. C. G. van Hespen, T. G. van Leeuwen, W. Steenbergen and S. Manohar, Biomed. Opt. Express, 2013, 4, 2555-2569.

22. G. Ku and L. V. Wang, Opt. Lett., 2005, 30, 507-509.

23. L. V. Wang and H.-I. Wu, Biomedical Optics: Principles and Imaging, John Wiley \& Sons, Inc., New Jersey, 2009.

24. C. Kim, C. Favazza and L. V. Wang, Chem. Rev., 2010, 110, 2756-2782.

25. Y. Wang, X. Xie, X. Wang, G. Ku, K. L. Gill, D. P. O'Neal, G. Stoica and L. V. Wang, Nano Lett., 2004, 4, 1689-1692.

26. Y. S. Chen, W. Frey, S. Kim, K. Homan, P. Kruizinga, K. Sokolov and S. Emelianov, Opt. Express, 2010, 18, 8867-8878.

27. X. Yang, S. E. Skrabalak, Z. Y. Li, Y. Xia and L. V. Wang, Nano Lett., $2007,7,3798-3802$.

28. K. H. Song, C. Kim, C. M. Cobley, Y. Xia and L. V. Wang, Nano Lett., $2009,9,183-188$

29. D. Pan, M. Pramanik, A. Senpan, S. Ghosh, S. A. Wickline, L. V. Wang and G. M. Lanza, Biomaterials, 2010, 31, 4088-4093.

30. D. Pan, M. Pramanik, S. A. Wickline, L. V. Wang and G. M. Lanza, Contrast Media Mol. Imaging, 2011, 6, 378-388.

31. A. De la Zerda, C. Zavaleta, S. Keren, S. Vaithilingam, S. Bodapati, Z. Liu, J. Levi, B. R. Smith, T. J. Ma, O. Oralkan, Z. Cheng, X. Chen, H. Dai, B. T. 
Khuri-Yakub and S. S. Gambhir, Nat. Nanotechnol., 2008, 3, 557-562.

32. M. Pramanik, M. Swierczewska, D. Green, B. Sitharaman and L. V. Wang, J. Biomed. Opt., 2009, 14, 034018.

33. G. Ku, M. Zhou, S. Song, Q. Huang, J. Hazle and C. Li, ACS nano, 2012, 6, $7489-7496$.

34. E. V. Shashkov, M. Everts, E. I. Galanzha and V. P. Zharov, Nano Lett., $2008,8,3953-3958$.

35. X. Wang, G. Ku, M. A. Wegicl, D. J. Bornhop, G. Stoica and L. V. Wang, Opt. Lett., 2004, 29, 730-732.

36. J. Xia, J. Yao and L. V. Wang, Electromagn Waves (Camb). 2014, 147, $1-22$.

37. J. F. Lovell, C. S. Jin, E. Huynh, H. Jin, C. Kim, J. L. Rubinstein, W. C. Chan, W. Cao, L. V. Wang and G. Zheng, Nat. Mater., 2011, 10, 324-332.

38. Z. Zha, Z. Deng, Y. Li, C. Li, J. Wang, S. Wang, E. Qu and Z. Dai, Nanoscale, 2013, 5, 4462-4467.

39. L. Cheng, C. Wang, L. Z. Feng, K. Yang and Z. Liu, Chem. Rev., 2014, 114, 10869-10939.

40. D. Jaque, L. M. Maestro, B. del Rosal, P. Haro-Gonzalez, A. Benayas, J. L. Plaza, E. M. Rodriguez and J. G. Sole, Nanoscale, 2014, 6, 9494-9530.

41. J. van der Zee, Ann. Oncol., 2002, 13, 1173-1184.

42. M. Chen, S. Tang, Z. Guo, X. Wang, S. Mo, X. Huang, G. Liu and N. Zheng, Adv. Mater., 2014, 26, 8210-8216.

43. L. W. Zhang, S. Gao, F. Zhang, K. Yang, Q. J. Ma and L. Zhu, Acs Nano, 
$2014,8,12250-12258$.

44. Y. S. Jin, Y. Y. Li, X. B. Ma, Z. B. Zha, L. L. Shi, J. Tian and Z. F. Dai, Biomaterials, 2014, 35, 5795-5804.

45. H. Moon, D. Kumar, H. Kim, C. Sim, J.-H. Chang, J.-M. Kim, H. Kim and D.-K. Lim, ACS nano, 2015, 9, 2711-2719.

46. X. F. Yan, H. Hu, J. Lin, A. J. Jin, G. Niu, S. L. Zhang, P. Huang, B. Z. Shen and X. Y. Chen, Nanoscale, 2015, 7, 2520-2526.

47. J. Shah, S. Park, S. Aglyamov, T. Larson, L. Ma, K. Sokolov, K. Johnston, T. Milner and S. Y. Emelianov, J. Biomed. Opt., 2008, 13, 034024.

48. P. Huang, J. Lin, W. Li, P. Rong, Z. Wang, S. Wang, X. Wang, X. Sun, M. Aronova, G. Niu, R. D. Leapman, Z. Nie and X. Chen, Angew. Chem. Int. Ed. , 2013, 52, 13958-13964.

49. D. Chen, C. Wang, X. Nie, S. Li, R. Li, M. Guan, Z. Liu, C. Chen, C. Wang, C. Shu and L. Wan, Adv. Funct. Mater.. 2014, 24, 6621-6628.

50. L. Cheng, J. Liu, X. Gu, H. Gong, X. Shi, T. Liu, C. Wang, X. Wang, G. Liu, H. Xing, W. Bu, B. Sun and Z. Liu, Adv. Mater., 2014, 26, 1886-1893.

51. W. Li, P. Rong, K. Yang, P. Huang, K. Sun and X. Chen, Biomaterials, $2015,45,18-26$.

52. K. Pu, A. J. Shuhendler, J. V. Jokerst, J. Mei, S. S. Gambhir, Z. Bao and J. Rao, Nat. Nanotechnol., 2014, 9, 233-239.

53. G. Qian, B. Dai, M. Luo, D. Yu, J. Zhan, Z. Zhang, D. Ma and Z. Y. Wang, Chem. Mater., 2008, 20, 6208-6216.

54. H. S. Oh, T.-D. Kim, Y.-H. Koh, K.-S. Lee, S. Cho, A. Cartwright and P. 
N. Prasad, Chem. Commun., 2011, 47, 8931-8933.

55. J. D. Yuen, J. Fan, J. Seifter, B. Lim, R. Hufschmid, A. J. Hecger and F. Wudl, J. Am. Chem. Soc., 2011, 133, 20799-20807.

56. J. D. Yuen, R. Kumar, D. Zakhidov, J. Seifter, B. Lim, A. J. Heeger and F. Wudl, Adv. Mater., 2011, 23, 3780.

57. J. Fan, J. D. Yuen, W. Cui, J. Seifter, A. R. Mohcbbi, M. Wang, H. Zhou, A. Heeger and F. Wudl, Adv. Mater., 2012, 24, 6164-6168.

58. J. Fan, J. D. Yuen, M. Wang, J. Seifter, J.-H. Seo, A. R. Mohebbi, D. Zakhidov, A. Heeger and F. Wudl, Adv. Mater., 2012, 24, 2186-2190.

59. Y. Wang, T. Kadoya, L. Wang, T. Hayakawa, M. Tokita, T. Mori and T. Michinobu, J. Mater. Chem. C, 2015, 3, 1196-1207.

60. S. Huang, R. K. Kannadorai, Y. Chen, Q. Liu and M. Wang, Chem. Commun., 2015, 51, 4223-4226.

61. J. Liu, J. Geng, L.-D. Liao, N. Thakor, X. Gao and B. Liu, Polym. Chem., 2014, 5, 2854-2862.

62. L. Nie, M. Chen, X. Sun, P. Rong, N. Zheng and X. Chen, Nanoscale, 2014, 6, 1271-1276.

63. J. D. Yuen, M. Wang, J. Fan, D. Sheberla, M. Kemei, N. Banerji, M. Scarongella, S. Valouch, T. Pho, R. Kumar, E. C. Chesnut, M. Bendikov and F. Wudl, J. Polym. Sci. A Polym. Chem., 2015, 53, 287-293.

64. D. K. Roper, W. Ahn and M. Hocpfner, The Journal of Physical Chemistry $C, 2007,111,3636-3641$.

65. Y. Liu, K. Ai, J. Liu, M. Deng, Y. He and L. Lu, Advanced Materials, 
2013, 25, 1353-1359.

66. M. Malmsten, Soft Matter, 2006, 2, 760-769.

67. C. Gong, S. Shi, P. Dong, B. Kan, M. Gou, X. Wang, X. Li, F. Luo, X. Zhao, Y. Wei and Z. Qian, Int. J. Pharm., 2009, 365, 89-99.

68. C. Lu, L. Liu, S.-R. Guo, Y. Zhang, Z. Li and J. Gu, European Polymer Journal, 2007, 43, 1857-1865.

69. Y. Liu, K. Ai, J. Liu, M. Deng, Y. He and L. Lu, Adv. Mater., 2013, 25, 1353-1359.

70. P. K. Upputuri and M. Pramanik, Biomed. Opt. Express, 2015, 6, $4118-4129$. 


\section{Chapter 7 Summary and Outlook}

\subsection{Summary}

In this thesis, the main purpose of my research is trying to design and synthesize multifunctional polymer nanoparticles and explore their potential applications in cancer imaging and therapy, in order to solve current problems and explore novel materials in biomedical application.

In chapter 2, the fluorescence intensity in aggregated state and photostability has been improved by synthesizing a well-defined polymer polycaprolactone (PCL) consisting of fluorophore covalently linked in the middle of the polymer chain. Moreover, due to exist of biodegradable PCL, the polymer showed non-toxic and good biocompatibility in cell study. Furthermore, these highly fluorescent nanoparticles exhibited significantly enhanced photostability in cell imaging and long-term tracing.

In chapter 3 , based on previous concept, a series of fluorescent PCL polymers with various light emission wavelengths ranging from visible to near-infrared (NIR) region in solid states were explored. The water-borne ink consisting of the PCL-dye-PCL colloidal nanoparticles with bright fluorescence and remarkable photostability can be used in macro-scale stamping, brush writing and micro-contact patterning. These unique properties make these fluorescent PCL-dye-PCL promising biomaterials in tissue engincering. Moreover, I have demonstrated that NIR-PCL with relatively strong NIR 
fluorescence and deep light penetration depth is a promising biopolymer for bioimaging and monitoring the degradation of PCL scaffolds in a non-invasive way.

Then in chapter 4, I have incorporated fluorescence bioimaging into amphiphilic block copolymers as nanocarrier. I found that the amorphous PDL with alkyl side chain would improve the encapsulation efficiency of anti-cancer drug than PCL. Meanwhile, the drug in this fluorescent polymer drug carricr can be quickly released under acidic condition. Moreover, the dye covalently bonded in the middle of polymer provides strong fluorescence signal for tracking polymers and drug. Therefore, the fluorescent amphiphilic block copolymers cannot only provide strong fluorescence tracking, but also enable to encapsulate anti-cancer drug in the hydrophobic core.

Except from the applications in bioimaging and drug carrier, in order to explore further applications of polymers nanoparticle with NIR absorbance, a series of NIR polymeric nanoparticles were designed and synthesized. In chapter $5, \mathrm{BBT}$ derivatives were synthesized to show strong absorbance over $800 \mathrm{~nm}$ and brightly emission over $1000 \mathrm{~nm}$ that could generate heat by converting light encrgy, which provides opportunitics of photothermal therapy. It has been successfully proved that BBT derivatives are alternative photothermal agents to gold materials.

Moreover, I further found that these materials present strong photoacoustic signal. The incorporation of photoacoustic imaging and photothermal therapy cnables these matcrials to become outstanding theranostic agents. Different to 
other theranostic materials that physically blend together, it incorporated imaging and therapeutic function into one material, which can avoid the separation of imaging and therapeutic agents in use.

\subsection{Outlook}

In future, I will carry out some further application of these multifunctional polymers. First, the biodistribution and pharmaceutical kinetics of NIR polymeric nanopartilcles should be investigated by animal model before clinical trial. Moreover, I also plan to study the treatment effect of the BBT based photothermal agents on mice with tumor. Second, I will implant NIR-PCL scaffold into animal model to track the degradation of PCL by NIR fluorescence non-invasively, as it has large potential in tissue engineering. Except from these, several future works are proposed as following:

\subsubsection{Amphiphilic block copolymer with NIR absorbance as}

\section{theranostic materials}




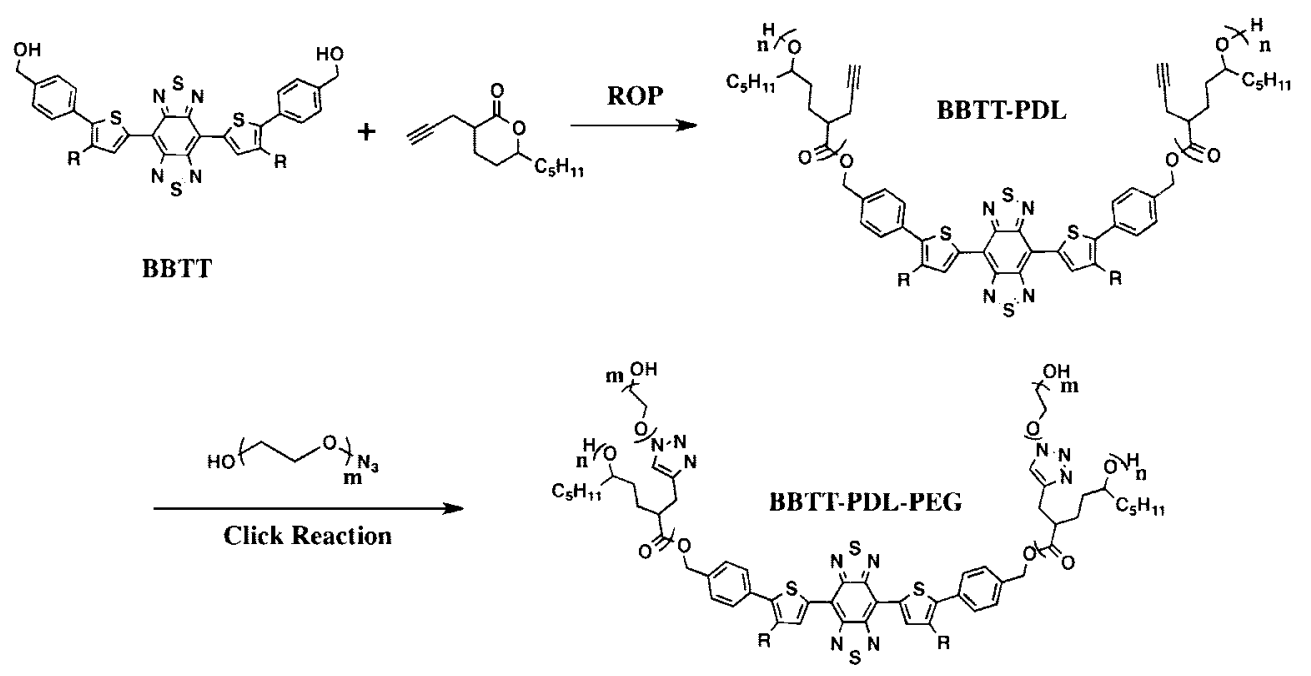

Scheme 7-1. Synthctic route of NIR amphiphilic block copolymers

I have synthesized the NIR dye (BBTT as shown in Scheme 7-1) and studied its photothermal effect in previous work, which showed high heat conversion efficiency. I try to design an amphiphilic block copolymer with strong NIR absorbance, which can be used not only to encapsulate some imaging probes or treatment drug, but also to provide photothermal effect Moreover, I can track the destiny of the treatment agents by using fluorescence or photoacoustic signal from this NIR polymer.

However, it will be biggest challerge that the BBT based molecule is sensitive to base condition, thus copper catalyst initiated ATRP is not suitable. We have to design a better synthetic route (Scheme 7-1) to avoid base condition by mild $\mathrm{CuSO}_{4}$ catalysed click reaction. Moreover, the functional group in side chain of polymer is easily modified with therapeutic molecules to form theranostic agents. 


\subsubsection{Metal-free light triggered radical polymerization using organic photocatalysts}

Controlled radical polymcrization has been widely used to synthesize block copolymer, however, metal catalyst copper is necessary in traditional ATRP reaction, which is not easily removed completely. The few amount of copper remaining in polymers could cause polymer properties changes and undesired toxicity in biological applications. Thus, non-metal catalyst based polymerization is an ideal alternative to figure out these concerns. Recently, some research groups have reported light induced controlled radical polymerization based on organic catalyst. In future, we proposed to use modified fluorescent dye with absorbance at $400 \mathrm{~nm}$ as initiator to start radical polymerization (Scheme 7-2) without any catalysts, in which neither catalysts nor by-products need to be removed after reaction.

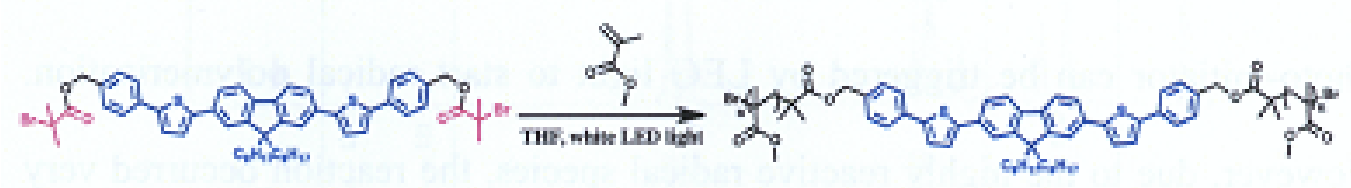

Scheme 7-2. Synthetic route of fluorescent PMMA by light triggered polymerization.

I try to carry out this experiment by using methyl methacrylate (MMA) monomer under LED light irradiation. After $3 \mathrm{~h}$ reaction and purification by precipitation in ether, the chemical structure of polymethyl methacrylate PMMA was confirmed by 'H-NMR (Figure 7-1 a), which showed the typical peaks from PMMA at $3.6 \mathrm{ppm}, 1.9 \mathrm{ppm}$ and 0.9-1.1 ppm. The consisting UV 
and RI trace of GPC (Figure 7-1 b) reveals high quality of polymcr, giving narrow PDI 1.6. When the reaction condition is changed, such as increased monomer concentration or nitrogen protection by freeze-pump, the molecular weight and PDI show no significant difference. I found that the polymerization cannot occur when without initiator or light irradiation, respectively, which indicated the photoinitiator and light are necessary component in this reaction.

To monitor the consuming of monomer in synthetic system, comparing the peaks of C-C double bond of monomer at 5.5-6.0 ppm and the methyl peaks from polymer around $1 \mathrm{ppm}$ from ${ }^{1} \mathrm{H}-\mathrm{NMR}$ (Figurc 7-2) as synthetic system, we can clearly find that the peaks of monomer decrease and the peaks from polymer increase with reaction time increasing. The GPC results (Figure 7-3) also reveal that the polymerization reaches highest molecular weight very quickly, but the conversion will increase with reaction time.

Thus, from the preliminary results, we can conclude that the photo-initiator can be triggered by LEG light to start radical polymerization. However, due to the highly reactive radical species, the reaction occurred very quickly. Next, we plan to optimize the experiments condition in order to control the molccular weight with increasing reaction time. Hopefully, well-defined multifunctional polymers synthesized by this method could present high quality in biological applications. 

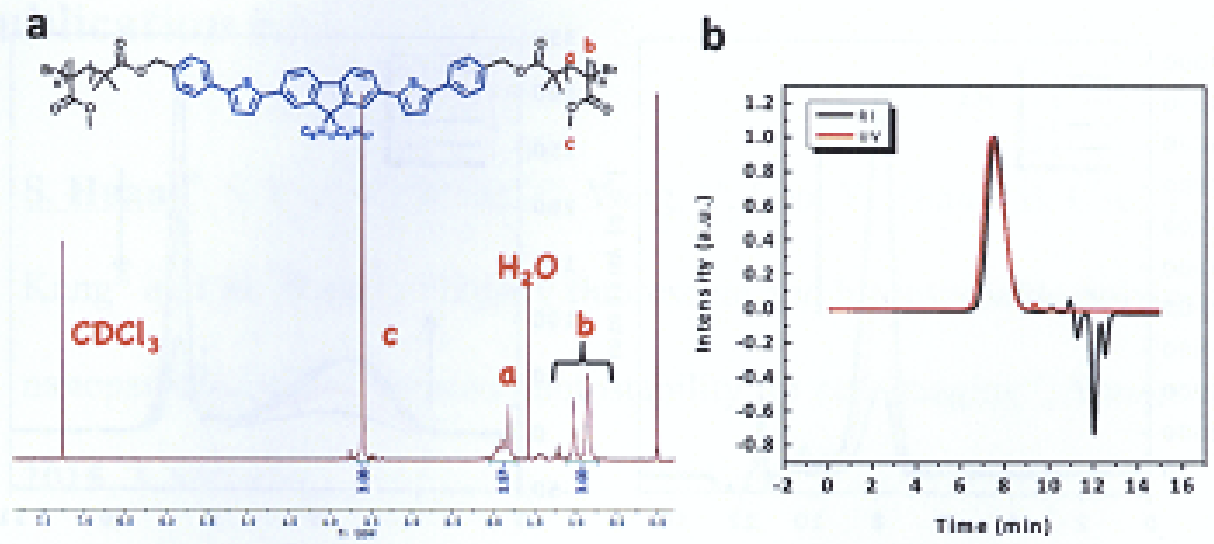

Figure 7-1. ${ }^{1} \mathrm{H}-\mathrm{NMR}$ spectrum $\left(\mathrm{CDCl}_{3}, 300 \mathrm{MHz}\right)$ of PMMA synthesized by light triggered polymerization.

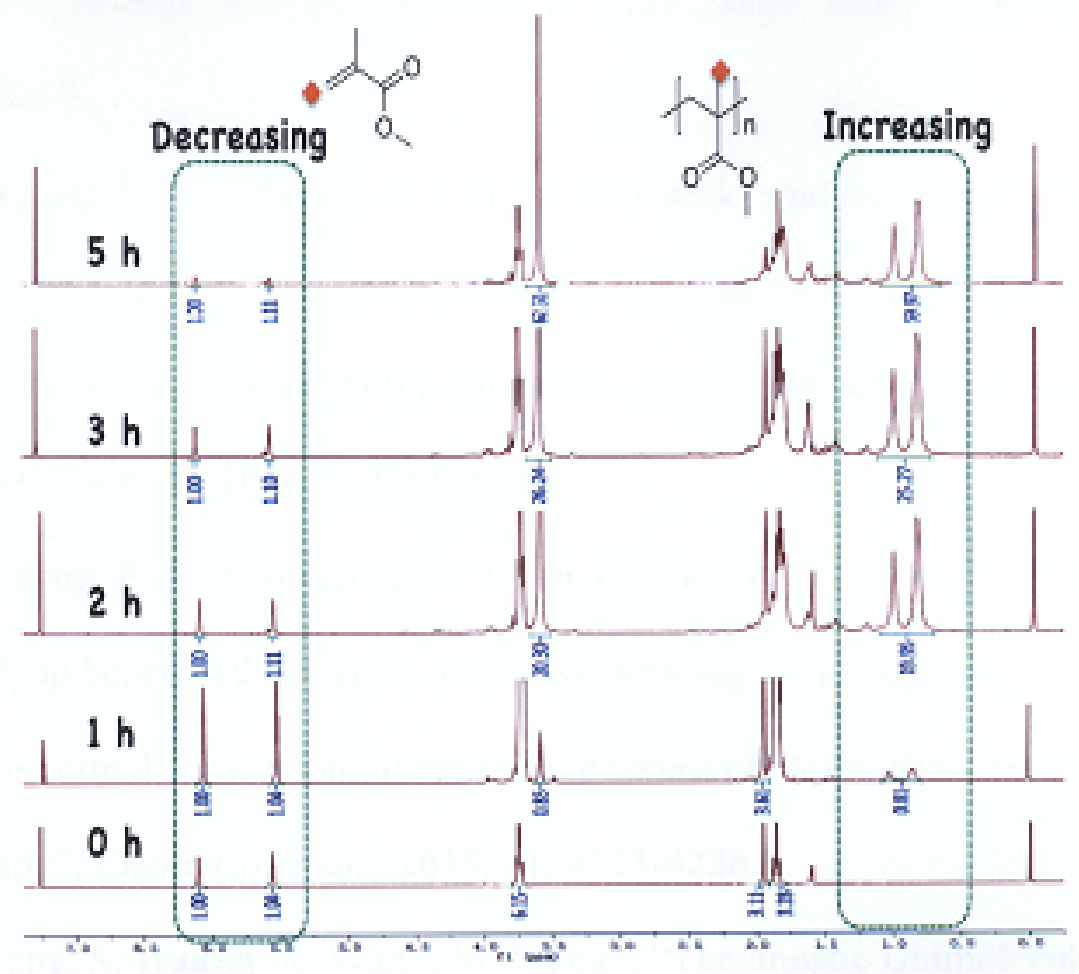

Figure 7-2. ${ }^{1} \mathrm{H}-\mathrm{NMR}$ result of monitoring the monomer conversion. 

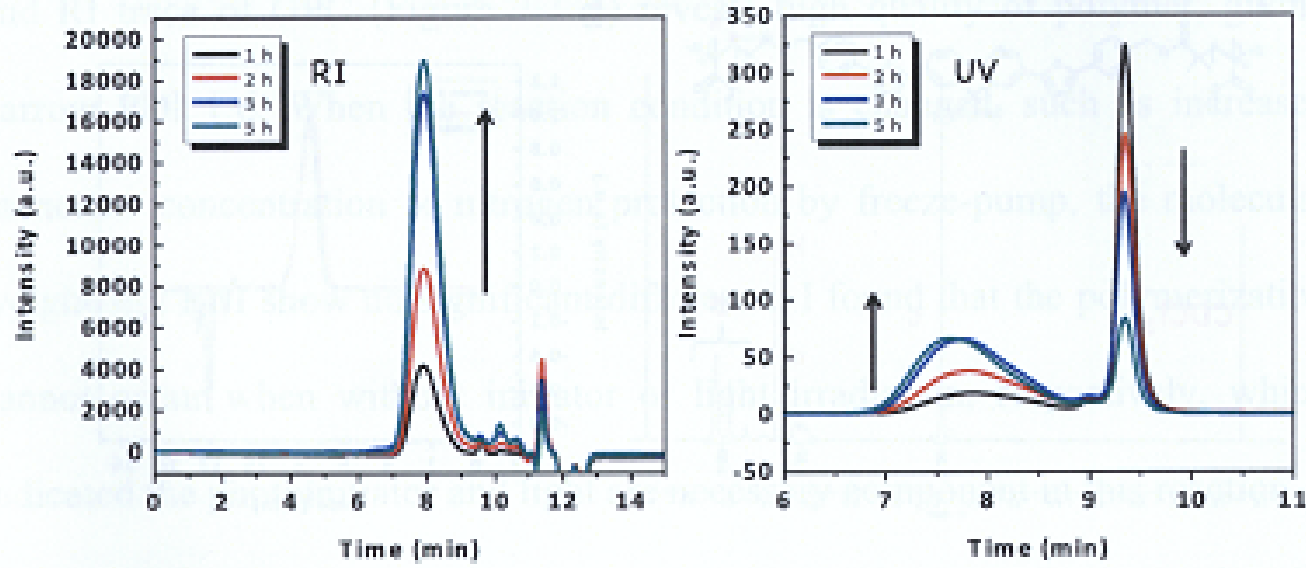

Figure 7-3. GPC traces show the evolution of $M_{\mathrm{w}}$ in the progress of photpinitiated polymerization. 


\section{Publication List}

1. S. Huang ${ }^{\#}$, S. Liu ${ }^{\#}$, K. Wang, C. Yang, Y. Luo, Y. Zhang, B. Cao, Y. Kang* and M. Wang*, "Highly fluorescent and bioresorbable polymeric nanoparticles with enhanced photostability for cell imaging", Nanoscale, 2015, 7, 889-895.

2. S. Huang, K. Wang, S. Wang, Y. Wang and M. Wang*, "Highly fluorescent polycaprolactones with tunable light emission wavelengths across visible to NIR spectral window", Adv. Mater. Interf., 2016, 3, 1600259

3. S. Huang, P. K. Upputuri, H. Liu, M. Pramanik* and M. Wang*, "A dual-functional benzobisthiadiazole derivative as an effective theranostic agent for near-infrared photoacoustic imaging and photothermal therapy“", $J$. Mater. Chem. B, 2016, 4, 1696-1703.

4. S. Huang, R. K. Kannadorai, Y. Chen, Q. Liu* and M. Wang*, "A narrow bandgap benzobisthiadiazole derivative with high near-infrared photothermal conversion efficiency and robust photostability for cancer therapy", Chem. Commun., 2015, 51, 4223-4226.

5. C. Yang, S. Huang, X. Wang, M. Wang*, "Theranostic Unimolecular Micclles of Highly Fluorescent Conjugated Polymer Bottlebrushes for Far Red/Near Infrared Bioimaging and Efficient Anticancer Drug Delivery", Polym. Chem. 2016, 7, 7455-7468 
6. J. Tao, S. Huang, C. Yang, M. Wang*, "Unimolecular Micelles of Amphiphilic Cyclodextrin-core Star-like Copolymers with Covalent pH-Responsive Linkage of Anticancer Prodrugs", Mol. Pharmaceutics 2016, DOI: 10.1021/acs.molpharmaceut.6b00708

7. H. Bohra, J. Shao, S. Huang, M. Wang*, "Facile synthesis of naphthodithiphenediimide based small molecules and polymers via direct arylation coupling", Tetrahedron Lett. 2016, 57, 1497-1501.

8. C. Yang, Q. T. Trinh, X. Wang, Y. Tang, K. Wang, S. Huang, X. Chen, S. H. Mushrif and M. Wang*, "Crystallization-induced red emission of a facilely synthesized biodegradable indigo derivative", Chem. Commun., 2015, 51, 3375-3378.

9. K. Wang, Y. Luo, S. Huang, H. Yang, B. Liu, M. Wang*, "Highly Fluorescent polycaprolactones decorated with di(thiophene-2-yl)-diketopyrrolopyrrole: A covalent strategy of tuning fluorescence properties in solid states", J. Poly. Sci. Part A: Poly. Chem., 2015, 53, 1032-1042. 